\title{
Taxonomic revision of Ceropegia (Apocynaceae: Ceropegieae) in India
}

\author{
Kambale S.S. ${ }^{1,2}$ \& S.R. Yadav ${ }^{2}$ \\ 'Department of Botany, Maratha Vidya Prasarak Samaj's Arts, Commerce \& Science College, \\ Tryambakeshwar, Maharashtra - 422 212, India. \\ ${ }^{2}$ Angiosperm Taxonomy Laboratory, Department of Botany, Shivaji University, Kolhapur, Maharashtra - 416004 , India. \\ *E-mail: skambalesu@gmail.com
}

\begin{abstract}
The genus Ceropegia (Apocynaceae: Ceropegieae) is revised for India based on field observation, literature survey and extensive herbarium studies. Sixty one taxa are recognized under seven sections. Nomenclatural anomalies are resolved. Distribution status is given for each species. A lectotype is designated for C. schumanniana and second step lectotypes are designated for C. hookeri and C. lucida; C. karulensis is reduced here as a variety of $C$. sahyadrica; $C$. mizoramensis and $C$. murlensis are reduced to the synonymy of $C$. longifolia subsp. sinensis. Detailed descriptions, notes, colour photoplates and taxonomic keys for sections, species and infra-specific taxa are presented.
\end{abstract}

Keywords: Apocynaceae, Ceropegia, Distribution, Endemism, Lectotypification, Taxonomy, Western Ghats.

\section{Introduction}

The genus Ceropegia L. comprises 244 species distributed in Africa and Madagascar, extending in the east to Arabia, India and China, northern part of Australia as far as Canary Islands (Bruyns, 2014). Highest diversity of the genus occurs in South Africa, followed by Kenya, Madagascar and India (Sri Rama Murthy et al., 2012). In India, the genus is represented by 53 species, 2 subspecies and 6 varieties of which 37 are endemic to Peninsular India (Ahmedullah \& Nayar, 1987). Majority of Ceropegia species in India occurs along steep hill slopes amidst Strobilanthes callosa Nees, several others in rock crevices at low to high elevation lateritic plateaus, along with bushes, forest margins, grasslands of dry deciduous forests, shola forest margins and still others prefer to grow at drier habitats (Figs. $1 \& 2$ ).

Received: 12.11.2018; Revised \& Accepted: 15.03.2019

Published Online: 31.03.2019
The genus Ceropegia can be easily distinguished from other genera of Ceropegieae viz. Brachystelma R.Br. and Caralluma R.Br. by its cage-like structure of flowers formed by corolla lobes, which are apically connate to various degrees (Hooker, 1883; Huber, 1957; Ansari, 1984; Li et al., 1995; Meve, 2009; Kullayiswamy et al., 2013; Kidyoo \& Paliyavuth, 2017).

Subsequent to the revision of Ceropegia in India (Ansari, 1984), a number of new species have been described from different parts of the country by various authors (Swarupanandan \& Mangaly, 1992; Sreekumar et al., 1998; Daniel \& Umamaheshwari, 2001; Yadav et al., 2004; Malpure et al., 2006; Punekar et al., 2006; Yadav et al., 2006; Yadav \& Shendange, 2010; Diwakar \& Singh, 2011; Kambale \& Yadav, 2012; Kullayiswamy et al., 2013; Rahangdale \& Rahangdale, 2012; Sujanapal et al., 2013; Manudev et al., 2016).

The present study is a comprehensive treatment of Ceropegia, based on extensive field surveys throughout its distributional range in India. The descriptions of majority of the species are based on live collections.

\section{Materials and Methods}

The specimens were collected from different regions of India. Live plants were grown in earthen pots in the Botanic Garden of Shivaji University, Kolhapur. Herbarium specimens were prepared following Vogel (1987) and Forman and Bridson (1992). Plants were photographed with Nikon D80/D90 DSLR cameras and photomicrographs using Zeiss Stemi SV11 Stereo microscope attached with a Nikon Coolpix 4500 camera. All voucher specimens were deposited in the herbarium of Shivaji University, Kolhapur (SUK). Digital images of the type specimens at 

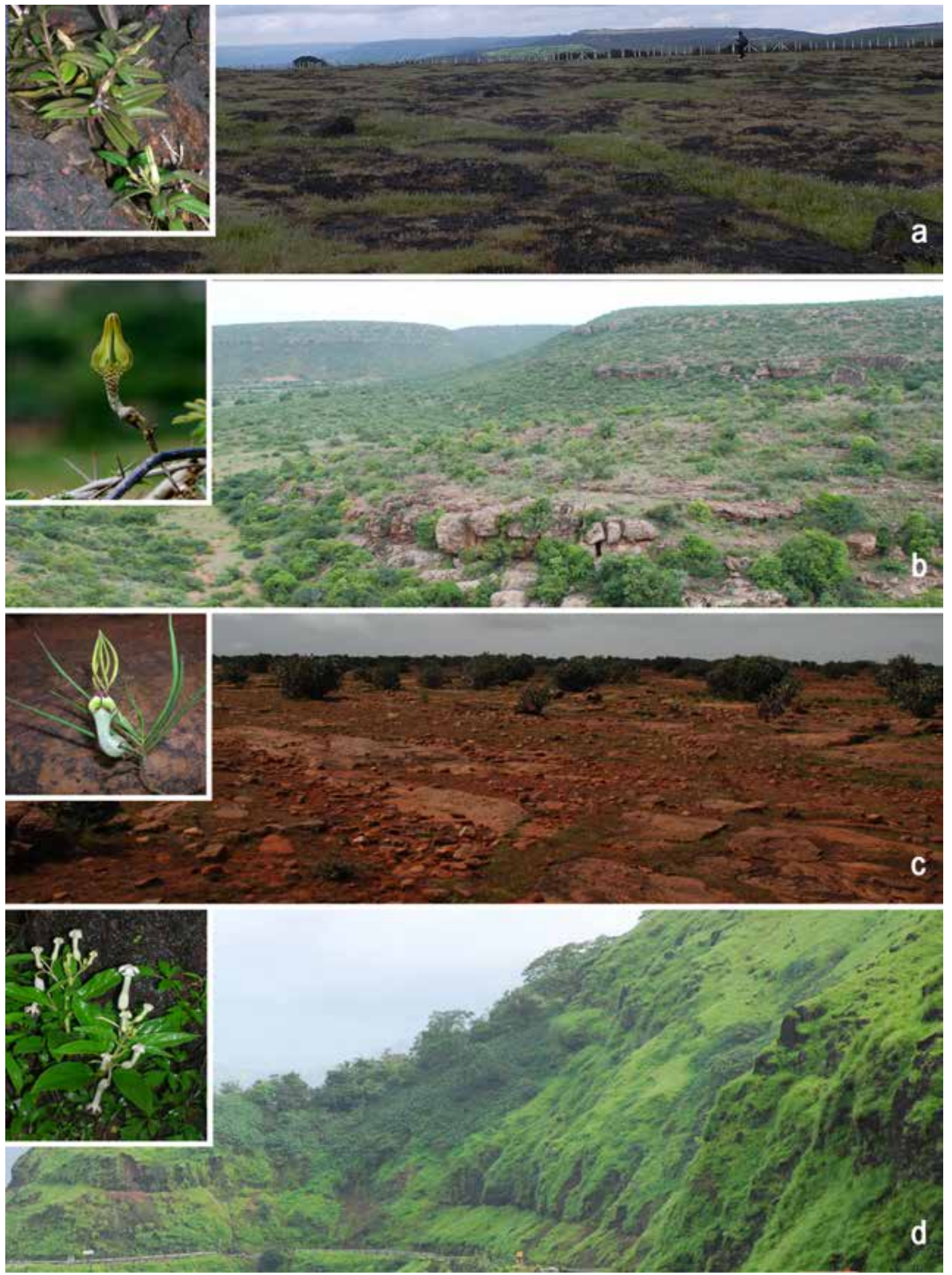

Fig. 1. Habitat of Ceropegia species: a. High elevation lateritic plateaus; b. Dry-deciduous scrub forest; c. Scrub open rocky areas dominated by succulent Euphorbias; $\mathbf{d}$. Steep mountain slopes (inset shows typical representative species of the habitats: a. C. jainii Ansari \& B.G. Kulk.; b. C. juncea Roxb.; c. C. spiralis Wight; d. C. sahyadrica Ansari \& B.G. Kulk. var. sahyadrica). 

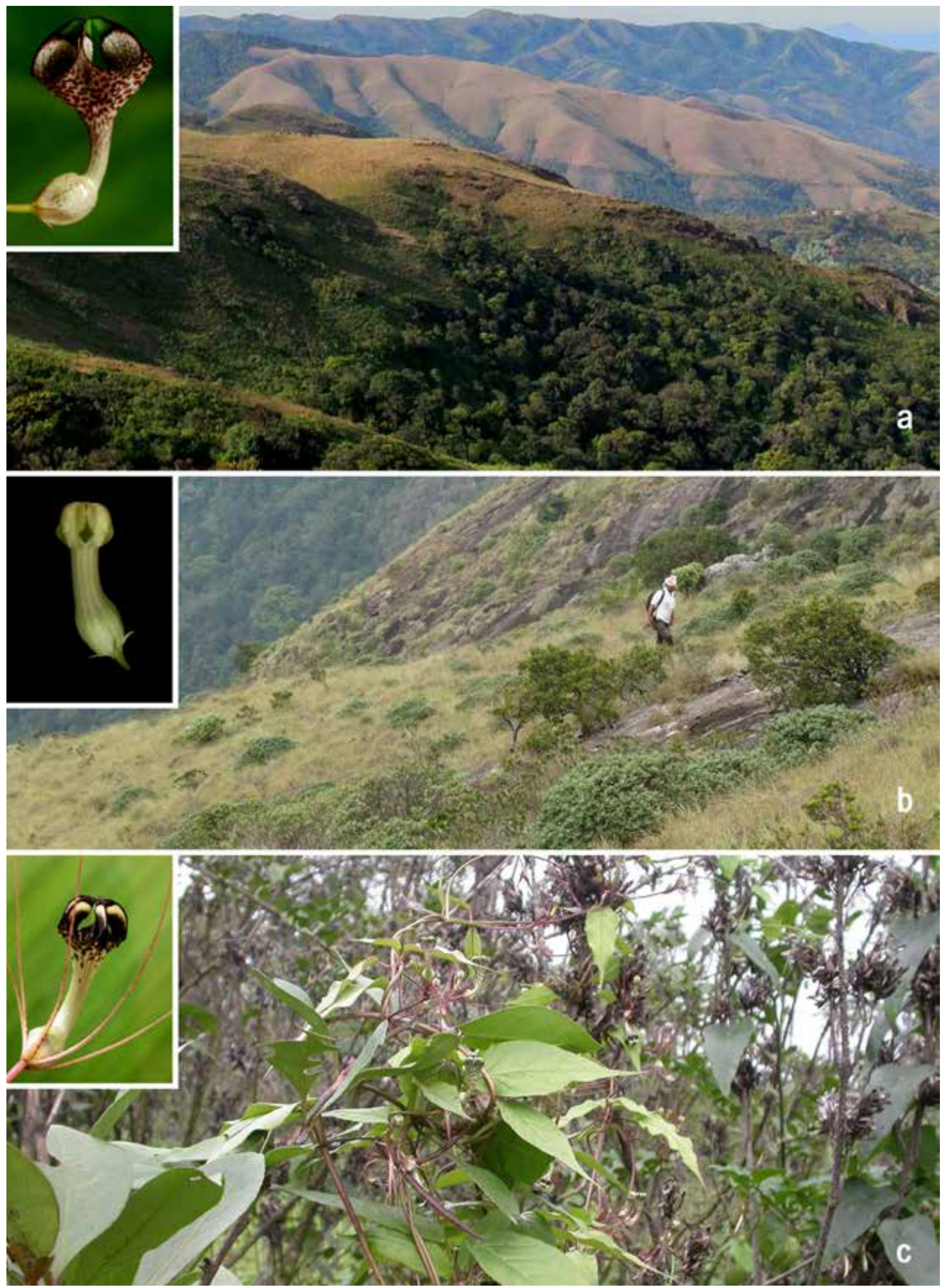

Fig. 2. Habitat of Ceropegia species: a. Shola forests associated with grasslands; $\mathbf{b}$. Grasslands at high elevations with less rainfall; c. Bushes of Strobilanthes callosa (inset shows typical species of the habitat: a. C. gardneri Thwaites; b. C. ravikumariana Kambale \& Gnanasek.; c. C. fantastica Sedgw.). 
BM, E, G, GH, PDA and $\mathrm{K}$ were examined. The specimens from the following herbaria: ARUN, ASSAM, BLAT, BAMU, BSI, BSID, CAL, CALI, FRLH, MH, PBL, KFRI, RHT, SUK, SKU, TBGT and Goa University herbarium were also consulted.

\section{Taxonomic treatment}

Ceropegia L., Sp. Pl. 1: 211. 1753; Roxb., Pl. Coromandel 1: 11, t. 7. 1795; Wight, Contr. Bot. India 30. 1834; B. Heyne ex Decne, Prodr. 8: 641. 1844; Wight, Icon. Pl. Ind. Orient. 4(1): 14. 1848; Benth. \& Hook.f., Gen. Pl. 2: 779. 1876; Hook.f., Fl. Brit. India 4: 66. 1883; K. Schum. in Engl. \& Prantl, Pflanzenr. 4(2): 270. 1895; H. Huber, Mem. Soc. Brot. 12: 20. 1957; Ansari, Fasc. Fl. India 16: 1. 1984; F. Albers \& Meve, Ill. Handb. Succ. P1. Asclepiadaceae 63. 2002. Type: Ceropegia candelabrum $\mathrm{L}$.

Vernacular names: Kandilpushp, Kharpudi (Marathi).

Perennial geophytic herbs. Rootstock tuberous or not; tubers depressed globose, globose-discoid; roots fibrous or fleshy. Stem prostrate, erect, twining or climbing, one or rarely $2-3$ from a single tuber, 0.1-1 $\mathrm{m}$ tall in erect species, 4-6 m tall in twining, terete or quadrangular, succulent or non-succulent, glabrous to sparingly hairy. Leaves simple, opposite-decussate, rarely whorled, sub-sessile to petiolate; lamina ovate, elliptic, ovate-lanceolate or broadly ovate, sometimes reduced to scales, succulent or non-succulent, glabrous or scabrous. Flowers solitary or few to many in umbellate cymes. Calyx 5-partite; sepals linear-subulate or triangular. Corolla tubular; tube abruptly, gradually or much dilated at the base, straight or curved, glabrous or hairy within at middle, with or without a ring of hairs at the mouth of dilated part, cylindrical or funnel-shaped at throat, equal or longer than corolla lobes; lobes linear, cordate, obovate or oblong, connate at tip, forming long conical, globose, ampuliform, ellipsoid cage, coiled, beaked or spirally twisted, rarely free, glabrous, finely pubescent or with glandular clavate, hollow clavate, conical uniseriate trichomes, usually multi-coloured, blotched or striped, reflexed along their back. Corona uni or bi-seriate, sessile or stipitate; outer of entire to bifid lobes, cupular, bowl or saucer-shaped, glabrous or ciliate along margins and within; inner if present of 5 erect to erecto-divergent, linear-clavate to spathulate lobes, alternate or opposite with outer corona, glabrous or stiffed hairy. Pollinarium erect; pollinia yellow to reddish, with pellucid margin, attached to red brown corpusculum by short caudicles. Follicles usually in pairs, rarely single, erect or horizontal, tapering to a fine point, glabrous. Seeds ovate-oblong, marginate, comose; coma white silky (Figs. $3 \& 4$ ).

Flowering \& fruiting: Throughout the year.

Distribution: 244 species in the world, distributed in Africa (except Mediterranean region), Canary Islands, India, Madagascar, New Guinea, northern Australia, southeast Asia and tropical Arabia (Good, 1952; Bruyns, 2003). Ceropegias are found at an elevation ranging from 16-2500 m. In India, majority of the species are distributed in peninsular part. Out of 61 Indian taxa, 44 (72\%) are endemic. All northeast Indian species (9), extend their distribution to neighboring countries. Highest diversity is found in Maharashtra with 25 species, of which 17 are endemic.

Notes: Majority of the Indian species are threatened due to the habitat modification and exploitation of tubers for food. A few species are even not collected after type collections.

\section{Key to the sections of Ceropegia in India}

1. Plants with well developed tubers ........................2

1. Plants without well developed tubers ................ 4

2. Tubers with fibrous roots ...................................

2. Tubers with fleshy roots............. sect. Ceropegia

3. Herbs geophytes, short, erect (except $C$. arnottiana) sect. Tiloris

3. Herbs climbing (except C. lawii, C. maccannii, C. panchganiensis, C. rollae, C. sahyadrica) sect. Indopegia

4. Leaves reduced to scales sect. Phalaena

4. Leaves well developed, not reduced to scales ...5 5. Leaves succulent. sect. Esculentae

5. Leaves not succulent 6

6. Roots fibrous sect. Janthina

6. Roots fleshy/swollen sect. Chionopegia 

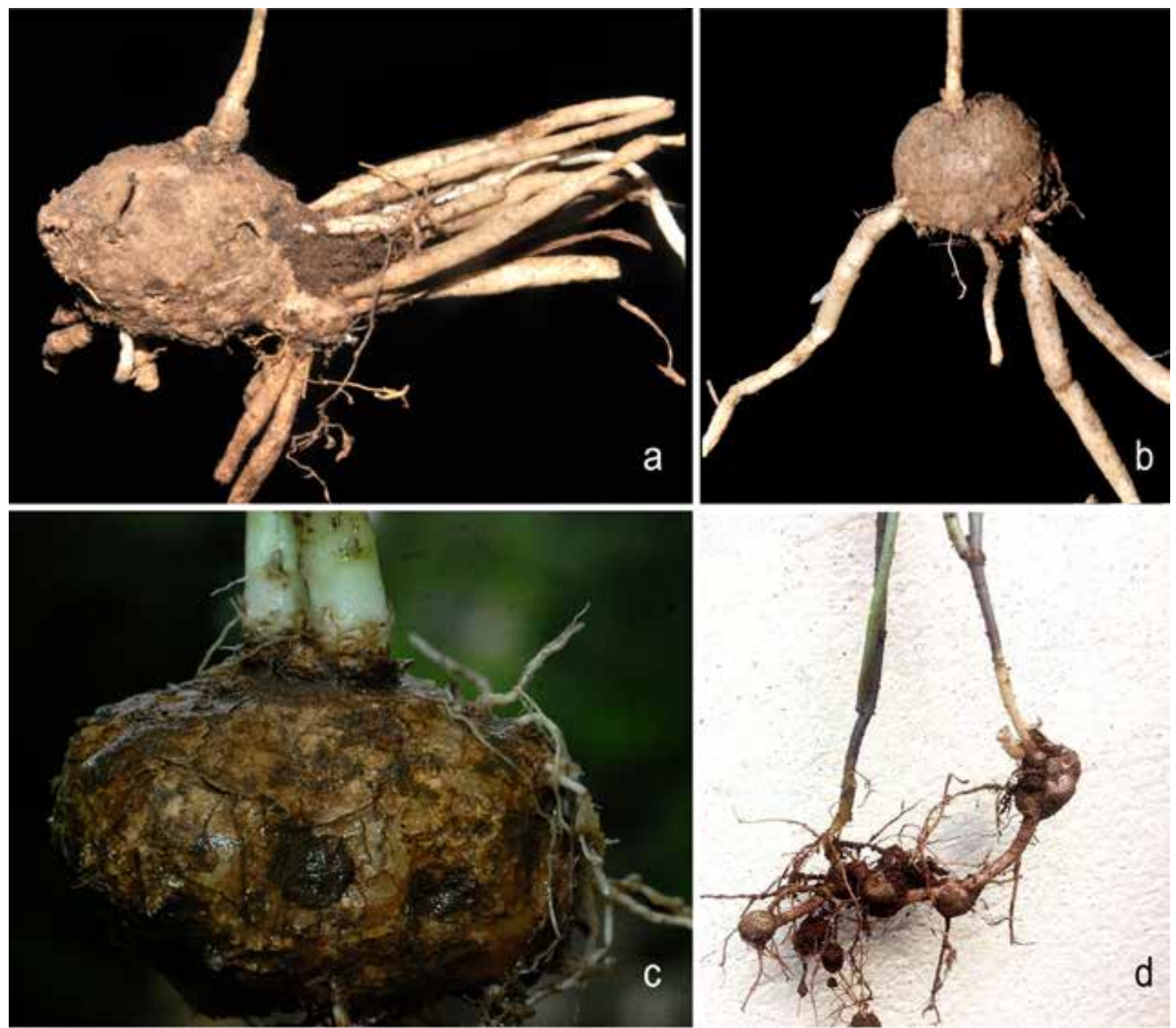

d
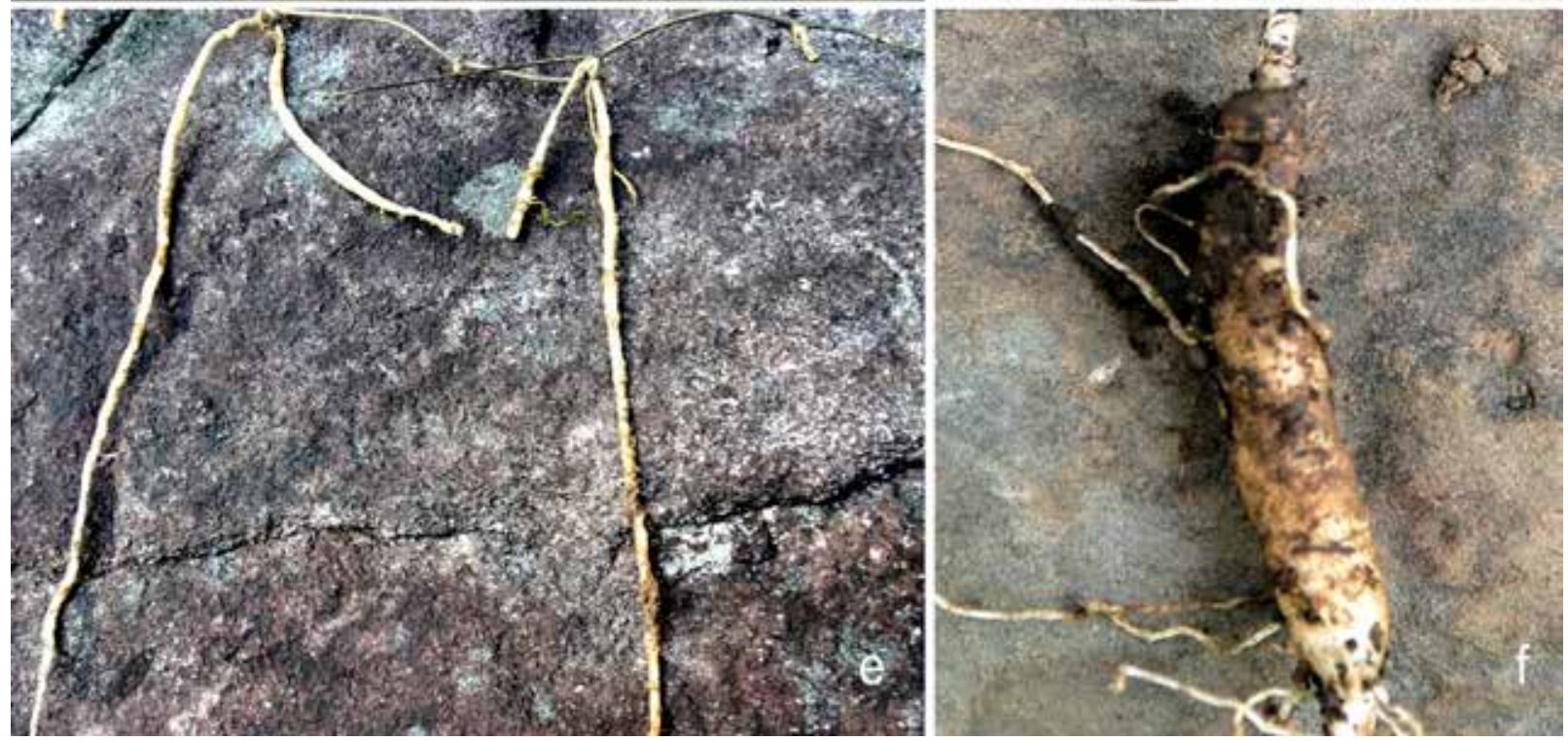

Fig. 3. Diversity of rootstock in Ceropegia species: a \& b. Globose tubers with fleshy roots in C. ravikumariana Kambale \& Gnanasek.; c. Globose tuber with fibrous roots in C. sahyadrica Ansari \& B.G. Kulk. var. sahyadrica; d \& e. Stolon like tubers in C. juncea Roxb. (d) \& C. intermedia Wight (e); f. Elongated tuber (probably the younger tuber) in C. odorata Nimmo ex J. Graham. (Photos a \& b. Navendu Page; d. H.C. Lingaraj). 


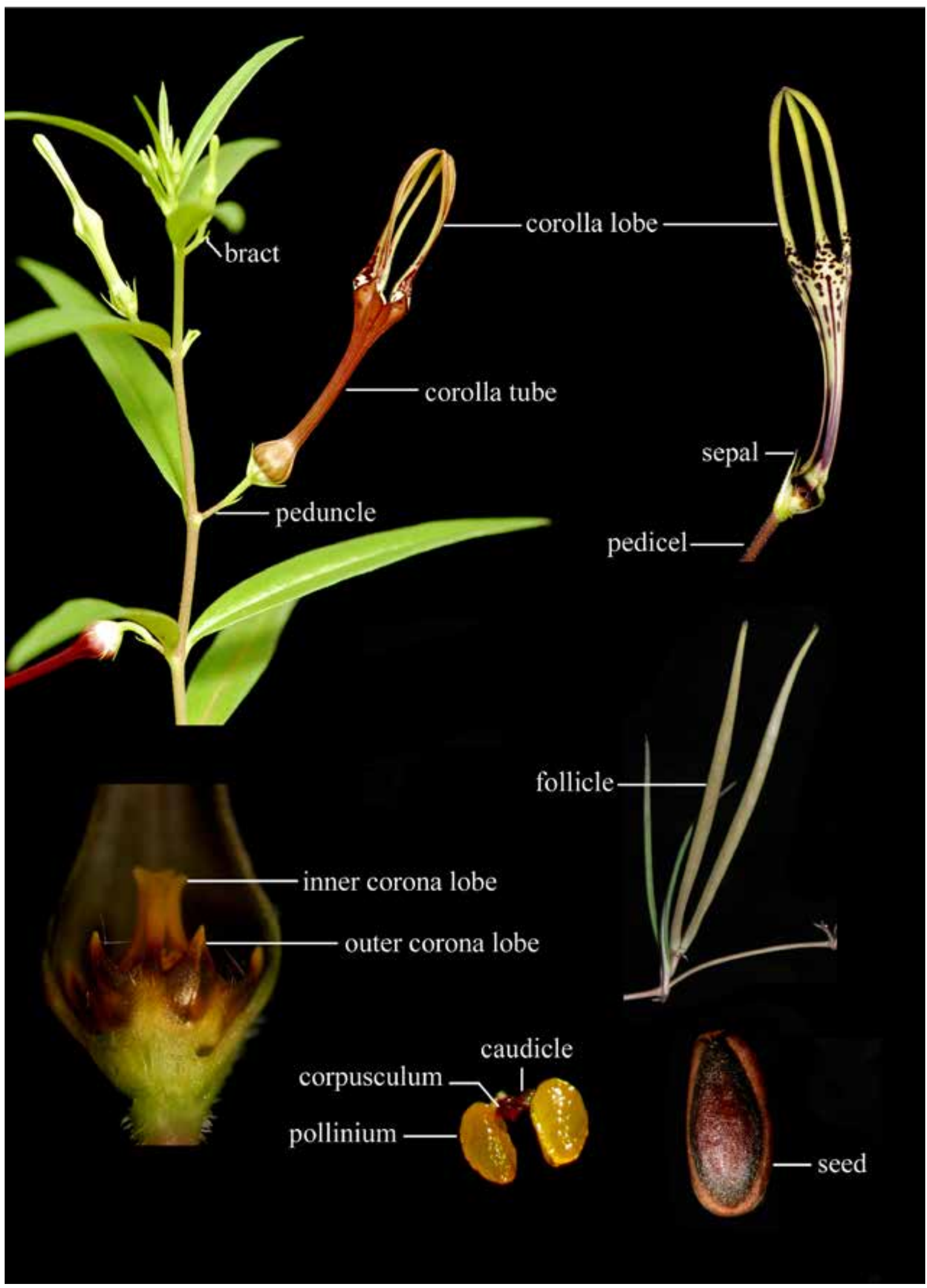

Fig. 4. General morphology of Ceropegia 
Table 1. Section-wise distribution of Ceropegia species (as per Bruyns et al., 2017)

\begin{tabular}{|c|c|}
\hline Section & Name of species \\
\hline \multirow{4}{*}{ Sect. Ceropegia } & C. candelabrum L. var. candelabrum \\
\hline & C. candelabrum L. var. biflora (L.) Ansari \\
\hline & C. mannarana Umamahesw. \& Daniel \\
\hline & C. ravikumariana Kambale \& Gnanasek. \\
\hline \multirow{9}{*}{ Sect. Chionopegia H. Huber } & C. angustifolia Wight \\
\hline & C. hookeri C.B. Clarke ex Hook.f. \\
\hline & C. kachinensis Prain \\
\hline & C. longifolia Wall. subsp. longifolia \\
\hline & C. longifolia subsp. sinensis $\mathrm{H}$. Huber \\
\hline & C. lucida Wall. \\
\hline & C. macrantha Wight \\
\hline & C. pubescens Wall. \\
\hline & C. wallichii Wight \\
\hline \multirow{2}{*}{ Sect. Esculentae Bruyns } & C. bulbosa Roxb. var. bulbosa \\
\hline & C. bulbosa Roxb. var. lushii (Graham) Hook.f. \\
\hline \multirow{22}{*}{ Sect. Indopegia H. Huber } & C. barnesii E.A. Bruce \& Chatterjee \\
\hline & C. beddomei Hook.f. \\
\hline & C. bhatii S.R. Yadav \& S.M. Shendage \\
\hline & C. ciliata Wight \\
\hline & C. ensifolia Bedd. \\
\hline & C. evansii McCann \\
\hline & C. fantastica Sedgw. \\
\hline & C. hirsuta Wight \& Arn. \\
\hline & C. huberi Ansari \\
\hline & C. lawii Hook.f. \\
\hline & C. maccannii Ansari \\
\hline & C. media (H. Huber) Ansari \\
\hline & C. oculata Hook. \\
\hline & C. odorata Nimmo ex J. Graham \\
\hline & C. omissa H. Huber \\
\hline & C. panchganiensis Blatt. \& McCann \\
\hline & C. pullaiahii Raja Kullayisw., Sandhyar. \& Karupp. \\
\hline & C. rollae Hemadri \\
\hline & C. sahyadrica Ansari \& B.G. Kulk. var. sahyadrica \\
\hline & $\begin{array}{l}\text { C. sahyadrica var. karulensis (Punekar, Tamhankar, Lakshmin., } \\
\text { Kumaran, Raut, S.K. Srivast. \& Kavade) Kambale \& S.R. Yadav }\end{array}$ \\
\hline & C. santapaui Wadhwa \& Ansari \\
\hline & C. vincifolia Hook. \\
\hline
\end{tabular}




\begin{tabular}{|l|l|}
\hline \multirow{5}{*}{ Sect. Janthina H. Huber } & C. andamanica Sreek., Veenakumari \& Prashanth \\
\cline { 2 - 2 } & C. decaisneana Wight \\
\hline C. elegans Wall. \\
\hline C. gardneri Thwaites \\
\hline C. intermedia Wight \\
\hline & C. maculata Bedd. \\
\hline C. manoharii Sujanapal, P.M. Salim, Anil Kumar \& Sasidh. \\
\hline C. metziana Miq. \\
\hline C. schumanniana Swarupan. \& Mangaly \\
\hline C. thwaitesii Hook. \\
\hline C. juncea Roxb. \\
\hline C. anantii S.R. Yadav, Sardesai \& S.P. Gaikwad \\
\hline C. anjanerica Malpure, M.Y. Kamble \& S.R. Yadav \\
\hline C. arnottiana Wight \\
\hline C. attenuata Hook. \\
\hline C. concanensis Kambale, Chandore \& S.R. Yadav \\
\hline C. fimbriifera Bedd. \\
\hline C. jainii Ansari \& B.G. Kulk. \\
\hline C. mahabalei Hemadri \& Ansari \\
\hline C. mohanramii S.R. Yadav, M.N. Gavade \& Sardesai \\
\hline C. nampyana Manudev, Kambale \& Pramod \\
\hline C. noorjahaniae Ansari \\
\hline C. pusilla Wight \& Arn. \\
\hline C. spiralis Wight \\
\hline
\end{tabular}

\section{Ceropegia sect. Ceropegia}

Climbing to creeping tuberous herbs. Tubers globose to irregular with fleshy roots. Lamina cordate to ovate-lanceolate or linear, glabrous; petiolate. Cymes c. 12-flowered. Corolla tube hairy within.

Distribution: Three species in the world, from southern Peninsular India to Sri Lanka. All the three species occur in India (Table 1).

\section{Key to the species of sect. Ceropegia}

1. Leaves linear-lanceolate C. ravikumariana

1. Leaves ovate, elliptic or ovate-elliptic

2. Corolla lobes oblong, glabrous at apex

C. mannarana

2. Corolla lobes ovate-lanceolate, hairy at apex ..... C. candelabrum
Ceropegia candelabrum L., Sp. Pl. 1: 211. 1753; Lour., Fl. Cochinch. 1: 114. 1790; B. Heyne ex Decne. in DC., Prodr. 8: 643. 1844; Thwaites, Enum. Pl. Zeyl. 199. 1860; Hook.f., Fl. Brit. India 4: 70. 1883; Gamble, Fl. Madras 4: 857. 1921; K.M. Matthew, Fl. Tamilnadu Carnatic 2: 935, t. 67d. 1983; B.D. Sharma et al., Fl. Karnataka, Analysis 166. 1984; Manilal, Fl. Silent Valley 176. 1988; K.M. Matthew, Fl. Palni Hills, S. India 2: 802. 1999; F. Albers \& Meve, Ill. Handb. Succ. Pl. Asclepiadaceae 71. 2002. Lectotype (designated by Huber, 1957): Plate of Rheede, Hortus Malabaricus 9: 27, t. 16.1673.

C. elliottii Hook.f., Fl. Brit. India 4: 70. 1883. Lectotype (designated by Kambale \& Yadav, 2015): colour drawing by W. Elliot.

C. discreta N.E. Br., Bull. Misc. Inform. Kew 262. 1909. Lectotype (designated by Kambale \& Yadav, 
2015): INDIA, Tamil Nadu, Coimbatore district, 1907, s.coll. s.n. (K000857815 image!).

Perennial glabrous twining or repent herbs. Rootstock tuberous or of fleshy roots; tubers reduced, c. $1 \mathrm{~cm}$ diam., , rooting at nodes. Stem c. $5 \mathrm{~m}$ long, slender, branched, glabrous. Lamina $4.6-8.6 \times 3.0-5.4 \mathrm{~cm}$, ovate or elliptic, acuminate at apex, truncate-rounded at base, glabrous or hairy along margins and nerves beneath, deep green above, pale below; petioles $1.7-3.7 \mathrm{~cm}$ long, channeled above, hairy along margins and on ventral side. Cymes umbellate c. 10-flowered, extra-axillary; peduncles $0.7-3.2 \mathrm{~cm}$ long, longer than pedicels, glabrous; bracts c. $1.4 \times 0.45 \mathrm{~mm}$, subulate, glabrous; pedicels $7-8 \mathrm{~mm}$ long, glabrous. Sepals c. $2.5 \times 0.95 \mathrm{~mm}$, subulate, purple tinged at apex, glabrous. Corolla $2.2-2.4 \mathrm{~cm}$ long; tube $1.8-1.9 \mathrm{~cm}$ long, slightly curved-straight, abruptly dilated at base, sub-cylindrical at throat, glabrous at lower half within and hairy at upperhalf, greyish striated with faint purple from base to upward; lobes 4-5 mm long, base ovate, connate at the tip forming globular cage, lobes reflexed on their back, hairy at the apex, yellowish-green, deep purple at the tip. Corona bi-seriate, stipitate; outer of 5 entire to tri-dentate lobes, bowl-shaped, c. 2 $\times 3 \mathrm{~mm}$, ciliate within and along margins, purple along margins, yellow-orange otherwise; inner of 5 erect, c. $2 \mathrm{~mm}$ long, connivent, spathulateclavate lobes, opposite with outer corona, deep purple at base, faint at apex. Pollinia c. $0.2 \times 0.2$ $\mathrm{mm}$, yellow-red. Follicles $c .12 \mathrm{~cm}$ long, straight, tapering towards both ends, glabrous. Seeds 12-15 $\times 1.1-1.5 \mathrm{~mm}$, brown, ovoid, comose; coma c. 2.5 $\mathrm{cm}$, long silky white.

\section{Key to the varieties}

1. Corolla lobes $4-5 \mathrm{~mm}$ long, not beaked. var. candelabrum

1. Corolla lobes $0.5-11 \mathrm{~mm}$ long, beaked var. biflora

Ceropegia candelabrum L. var. candelabrum; Ceropegia candelabrum L. var. candelabrum $\mathrm{H}$. Huber in Dassan. \& Fosberg, Revised Handb. Fl. Ceylon 4: 120. 1983; Ansari, Fasc. Fl. India 16: 11. 1984; Karthik. et al., Fl. Pl. India 1: 161. 2009; Kambale \& S.R. Yadav, Asklepios 115: 29. 2013.

Fig. 5

Vernacular name: Bitharige (Kannada).
Flowering \& fruiting: August-March.

Chromosome number: $2 n=22$ (Raghavan \& Ansari, 1975).

Habitat: Grows along slopes, road-sides in dryscrub and evergreen forests in association with Psychotria sp.

Distribution: India (Andhra Pradesh, Karnataka, Kerala, Tamil Nadu) and Sri Lanka.

Specimens examined: INDIA, Karnataka, way from Coorg to Talcauvery, 29.11.2010, S.S. Kambale E S.R. Yadav SUK 2556; Ibid. (grown in garden), 26.01.2012, S.S. Kambale 5276 (SUK!); Madapur, near Karekad Estate, 09.10.1961, A.S. Rao 75018 (BSI!); South Canara, 26.10.1900, C.A. Barber 2110 (MH!). Kerala, Idukki district, Meenmutty, 02.10.1983, C.N. Mohanan 79904 (MH!); Kannur district, Ezhimala, 26.10.1988, Nambiar E Sasidharan 2568 (KFRI!); Kollam district, Koni, 27.07.1978, C.N. Mohanan 58318 (CAL!); Palakkad district, near Mekaran, 06.09.1913, C.C.Calder \& M.S. Ramaswami 601 (CAL!); Parambikulam, 11.1910, A. Meebold 712401 (CAL!); Thiruvananthapuram district, Bonacaud Tea estate, s.d., J. Joseph 44535 (MH!); Ibid., 23.10.2013, S.S. Kambale \& S.R. Yadav SSK 96 (SUK!). Thrissur district, Mukkali, 28.11.1973, E. Vajravelu 44871; Peechi, KFRI Campus, 29.09.1978, N. Sasidharan 83 (KFRI!); SVNP, 02.10.1965, E. Vajravelu 26184 (MH!), Tamil Nadu, Chennai district, s.loc., 09.1884, J.S. Gamble 14837 (CAL!).

Ceropegia candelabrum L. var. biflora (L.) Ansari, Fasc. Fl. India 16: 12. 1984. Ceropegia biflora L., Sp. Pl. 1: 211. 1753; Trimen, Handb. Fl. Ceylon 3: 167. 1895. Lectotype (designated by Ansari, 1984): Plate of Hermann Herb. Ceropegia 110 (BM!).

Ceropegia tuberosa Roxb., Pl. Coromandel 1: 12. t. 9. 1795; Dalzell \& A. Gibson, Bombay Fl. 154. 1861; Hook.f., Fl. Brit. India 4: 70. 1883; T. Cooke, Fl. Bombay 2: 241. 1905; B.D. Sharma et al., Fl. Karnataka, Analysis 166. 1984. C. candelabrum L. subsp. tuberosa (Roxb.) H. Huber in Dassan. \& Fosberg, Revised Handb. Fl. Ceylon 4: 120. 1983; H.O. Saxena \& Brahmam, Fl. Orissa 2: 1087. 1995; Karthik. et al., Fl. Pl. India 1: 161. 2009; Kambale \& S.R. Yadav, Asklepios 115: 29. 2013. Lectotype (designated by Huber, 1983): Roxb., Pl. Corom. 1, t. 9.1795.

Ceropegia candelabrum auct. Hanumanthappa in 

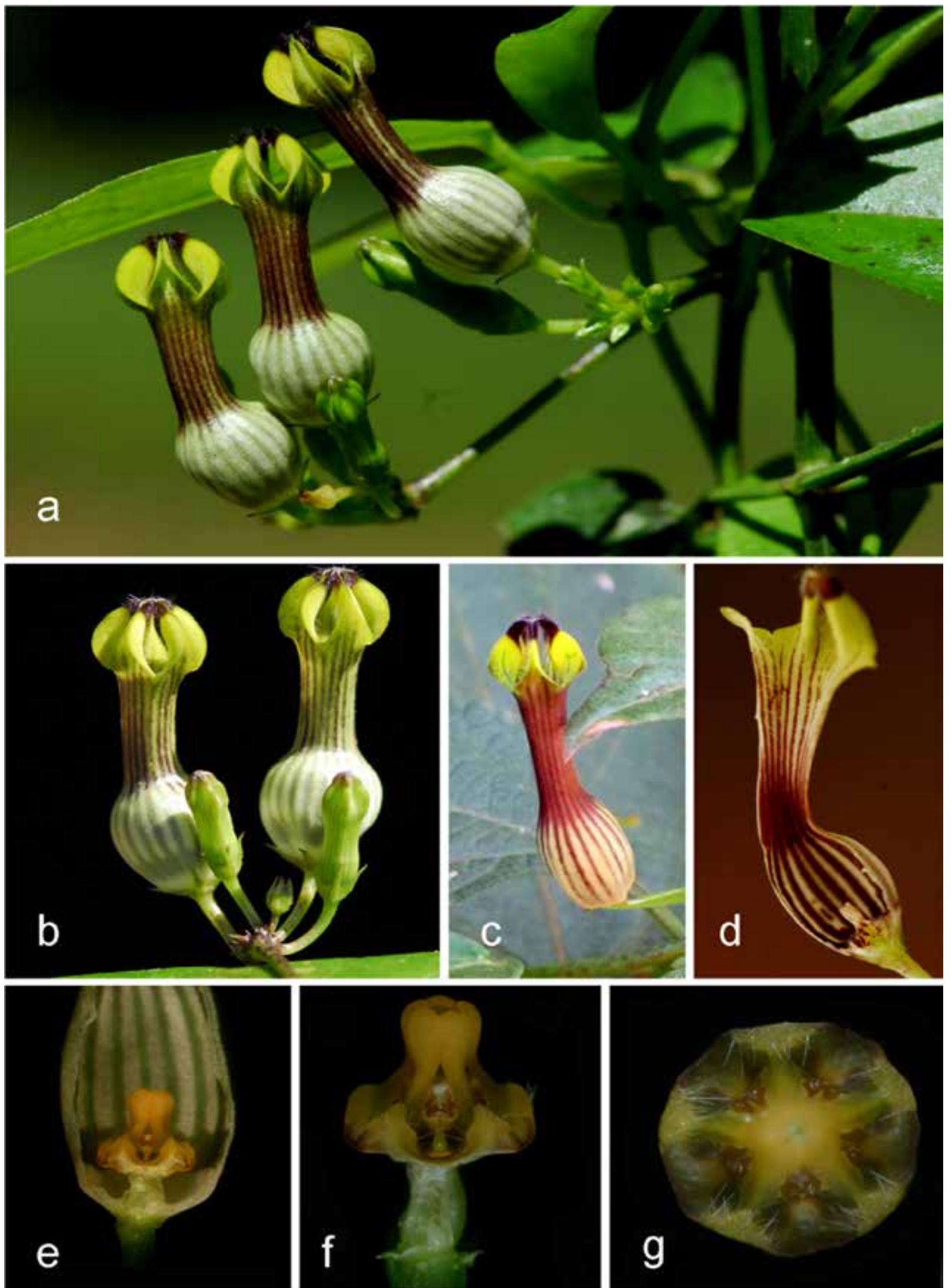

Fig. 5. Ceropegia candelabrum L. var. candelabrum: a. Habit with inflorescence; b. Inflorescence-close-up; c. Flower with reddish corolla tube; d \& e. Flower L.S.; f. Corona-lateral view; g. Corona-top view. 

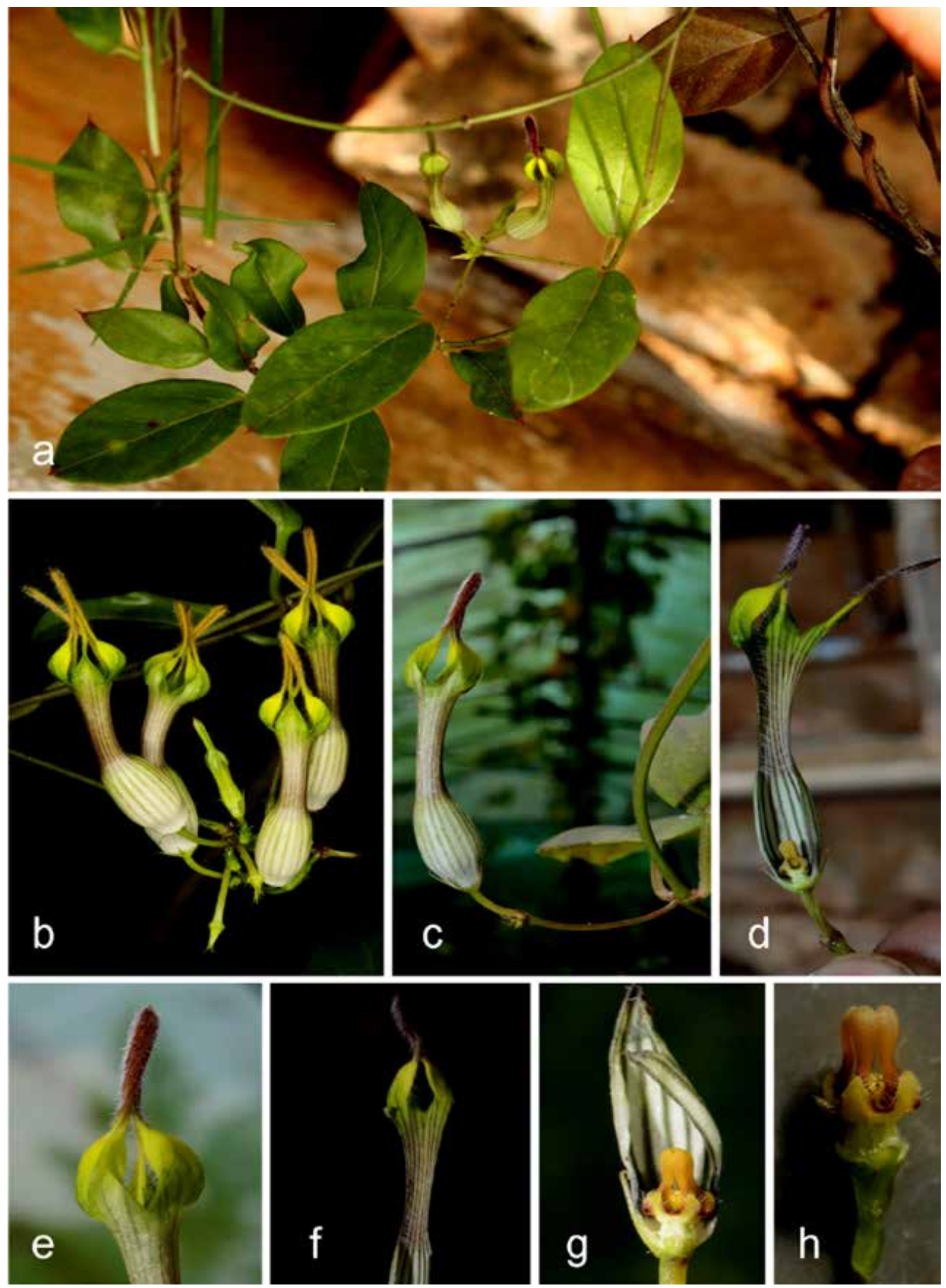

Fig. 6. Ceropegia candelabrum var. biflora (L.) Ansari: a. Habit; b. Inflorescence; c. Flower; d. Flower L.S.; e. Corolla lobes-close-up; f. Downwardly pointed hairs within corolla tube; $\mathbf{g} \& \mathbf{h}$. Corona. 
Pull. \& Moulali, Fl. Andhra Pradesh 2: 585. 1997; F. Albers \& Meve, Ill. Handb. Succ. Pl. Asclepiadaceae 71. 2002, p.p., non Linnaeus, 1753.

Fig. 6

Vernacular name: Wel-mottu (Sinhala).

Lamina ovate to elliptic. Cymes umbellate, $c$. 12-flowered, extra-axillary; peduncles $c .4 .5 \mathrm{~cm}$ long; pedicels c. $1.5 \mathrm{~cm}$ long. Sepals $3-4.5 \mathrm{~mm}$ long, linear. Corolla $2.5-3.5 \mathrm{~cm}$ long; tube 1.2-2.4 $\mathrm{cm}$ long, slightly curved, abruptly dilated at base, sub-cylindrical, funnel-shaped at throat, glabrous at lower half within and hairy with downwardly pointed hairs at upper-half, greyish coloured striated with faint purple from base to upward; lobes $0.5-1.1 \mathrm{~cm}$ long, broader at the base, linear above, coiled at the tip forming beaked structure, usually connate at the tip, rarely free, hirsute at the upper $1 / 3^{\text {rd }}$ portion, hairy within at the base, orange-deep purple at the upper $1 / 3^{\text {rd }}$ portion, yellowish-green at the base. Follicles 10-14 cm long.

Flowering \& fruiting: August-January.

Habitat: Grows along slopes and road-sides in dryscrub forests.

Distribution: India (Andhra Pradesh, Karnataka, Kerala, Orissa, Tamil Nadu) and Sri Lanka.

Specimens examined: INDIA, Andhra Pradesh, Chittoor district, Nagalapuram hills, 04.10.1974, M. Chandrabose 45232 (MH!); Cuddapah district, Talakona top, 25.11.2007, B.R. Rao \& B. Sadasivaiah FAP 30389 (SKU!); East Godavari district, stream near Devaramandu, 26.09.1980, G.V.Subbarao 68572 (MH!); Godavari district, Adatigala, 15.08.1914, M.S. Ramaswami 1698 (CAL!); Guntur district, Kendavendu fort, 18.10.1902, C.A. Barber CAB 4662 (MH!); Mehboobnagar district, Mannanur, 31.07.1995, T.D. Chandrakumar FAP 16805 (SKU!); Prakasam district, Tummalabailey, 08.10.1995, R. Vijaykumar FAP 15890 (SKU!); Srikakulam district, Rampa hills, 25.09.1920, V. Narayanaswami 283 (CAL!); Visakhapatnam district, Kothuru, 28.09.1961, N.P. Balakrishnan 886 (CAL!). Karnataka, Chamarajanagar district, way from Mysore to Gundlupet, 02.08.1964, B.D. Naithani 21308 (MH!); Kolar district, Kothapally, 26.06.1978, T.M. Ramakrishna TMR 1072 (MH!); Tumkur district, Devrayandurga, 15.08.1978, C.J. Saldanha KFP 2214 (CAL!). Kerala, Idukki district, Panamkutty, 17.10.1982, C.N. Mohanan 74647
(MH!); Kollam district, s.loc., 28.08.1913, M.R. Rao 2145 (CAL!); Wayanad district, s.loc., 1881, R.H. Beddome s.n. (MH!). Orissa, Ganjam district, Chilika lake, Dethatia Island, 09.09.1913, D. Hoopa 39567 (CAL!). Tamil Nadu, Coimbatore district, Marudhamalai, 05.11.1956, K.M. Sebastine 1205; Ibid., 27.11.2010, S.S. Kambale, A.R. Kulavmode \& R.V. Gurav SUK 2557 (SUK!); Mount Stuart, 27.10.1961, J. Joseph 13384 (CAL!); Dharmpuri district, Geratti forest, 25.07.1978, E. Vajravelu 57976 (MH!); Dindigul district, Sirumalai, 10.1951, s.coll. s.n. (RHT!); Madurai district, Madurai, 10.11.1911, C.E.C. Fischer 3177; way to Paichahur, 20.10.1977, M. Chandrabose 51395 (MH!); Nagapattinam district, Point Calimere, 19.01.1961, J. Ellis 11786 (MH!); Nilgiris district, Kunukkumadavu, 05.11.1970, E. Vajravelu 36715 (MH!); North Arcot district, Tippukadu Reserve forest, 20.11.1965, K. Ramamurthy 17611 (MH!); Ramanathapuram district, Sundharamadayan, 13.11.1989, V. Balsubramanian KASC 2199 (MH!); South Arcot district, Pakamalai Reserve forest, 10.09.1977, K. Ramamurthy 51150 (CAL!); Tirunelveli district, Courtallam, 30.11.1913, M.R. Rao 1913; Lower Kodayar, Vallachithodu, A.N. Henry 68936 (CAL!); Virudhunagar district, Nagariyar Estate, 11.11.1989, S.R. Srinivasan 86973 (MH!).

Conservation status: It is represented by good populations in drier parts of South India. However, records of its presence and range of distribution outside this area are not available. So the status is treated as Data Deficient (DD).

Notes: Ceropegia biflora was described by Linnaeus (1753) based on the drawing of Paul Hermann, while C. tuberosa Roxb. was described latter by Roxburgh in 1795. Huber (1957, 1983) reduced the former as a synonym and the latter as a variety of C. candelabrum. However, Ansari (1984) reduced C. tuberosa as a synonym under C. biflora and recognized the latter as a variety under $C$. candelabrum. At varietal rank, C. candelabrum var. biflora has priority and hence in the present study the treatment given by Ansari (1984) has been followed. This taxon closely resembles var. candelabrum but differs in having beaked linear corolla lobes.

Ceropegia mannarana P. Umam. \& P. Daniel, Fl. Gulf Mannar South. India 262. 2001. Type: INDIA, Tamil Nadu, Tirunelveli district, Gulf of Mannar 
coast, Uvari, 18.11.1995, S.A. Muthukumar 106332 (holo CAL!; iso MH!).

Perennial twining herbs. Stem sparsely hairy. Lamina $0.6-5.3 \times 0.3-2.6 \mathrm{~cm}$, elliptic-oblong to ovate, acute-cuspidate at apex, cuneate at base, ciliate along margins and beneath, petioles $1-2 \mathrm{~cm}$ long, channeled above, pilose. Cymes umbellate, 3-10-flowered, extra-axillary; peduncles $2-4 \mathrm{~cm}$ long, glabrous; pedicels 3-10 mm long, glabrous, rarely hairy. Sepals $2-3 \mathrm{~mm}$ long, linear, pubescent. Corolla $1.7-2.1 \mathrm{~cm}$ long, pale purplish violet; tube $1-1.4 \mathrm{~cm}$ long, straight, dilated at the base, hairy within at the throat; lobes 3-7 mm long, oblong, abruptly acute, connate at the tip, free at maturity, glabrous outside, hairy within at tip. Corona biseriate; outer of 5-bifid ciliate lobes, c. $2 \times 3 \mathrm{~mm}$; inner of 5 erect, spathulate lobes, c. $3 \mathrm{~mm}$ long. Pollinarium c. $0.2 \times 0.2 \mathrm{~mm}$. Follicles $c .9 \mathrm{~cm}$ long, linear, green with purplish dots.

Flowering \& fruiting: October-December.

Habitat: Grows in sandy soil, in association with Euphorbia antiquorum L.

Distribution: Endemic to Tamil Nadu, India.

Specimen examined: INDIA, Tamil Nadu, Gulf of Mannar Coast, Kanyakumari, Vivekanantha Kendra Campus, 21.11.1995, S.A. Muthukumar 106398 (paratype $\mathrm{MH} !)$

Conservation status: It is known from only one locality and is assessed as Data Deficient (DD).

Notes: Ceropegia mannarana closely resembles to C. candelabrum. Bruyns et al. (2017) merged this species under the latter. However, it differs from it in having hirsute habit and corolla lobes being oblong and glabrous outside at apex. Hence, it is treated here as a distinct species.

Ceropegia ravikumariana Kambale \& Gnanasek., Rheedea 26(1): 57. 2016. Type: INDIA, Tamil Nadu, Tirunelveli district, Naraikkadu, 08 $28^{\prime} 11.7^{\prime \prime}$ N, 77029'01.5” E, 1368 m, 10.09.2010, K. Ravikumar \& K. Murugan 110037 (holo MH!; iso FRLH!). Fig. 7

Perennial repent (rarely erect) to twining herbs. Rootstock tuberous; tubers $1.8-3.5 \mathrm{~cm}$ diam., globose or horizontally elongated; roots $2.5-9 \mathrm{~cm}$ long, fascicled, fleshy. Stem 20-100 cm long, 1-2 $\mathrm{mm}$ in diam., terete, nodes $1-6 \mathrm{~cm}$ long, usually branched, purple to greenish, glabrous. Lamina
2-3.7 $\times 0.3-0.6 \mathrm{~mm}$, linear-lanceolate, acute at apex, narrowed at base, ciliolate along margins and midnerve beneath, deep green above, pale below; petioles 2-7 mm long, channeled above, puberulous in young leaves, glabrous otherwise. Inflorescence 2-flowered extra-axillary; peduncles 2-5 mm long, slender, glabrous; bracts 2, 1.2-2 mm long, linear; pedicels 3-8.5 mm long, slender, glabrous. Sepals 2-3.5 mm long, subulate, glabrous. Corolla 9-15 $\mathrm{mm}$ long, straight, glabrous, whitish-yellow; tube $5-10 \times$ c. $2 \mathrm{~mm}$, straight or slightly curved, much dilated at base, with downwardly pointed trichomes within, trichomes absent at the base, striated with purple lines; lobes 5, ovate-lanceolate, 2.5-6 $\times c$. $2.8 \mathrm{~mm}$, connate at the tip forming sub-globose cage, slightly reflexed along the margin, glabrous exterior, hairy within with downwardly pointed hairs, yellowish. Corona bi-seriate, $4-4.5 \mathrm{~mm}$ long; outer of 5 triangular to undulate lobes, saucershaped, slightly reflexed and hairy along margins and within, yellow margined with purple; inner of 5 linear lobes, c. 1.4-3 mm long, erect, spathulate, opposite to outer corona, yellow. Pollinarium c. 0.2 $\times 0.4 \mathrm{~mm}$. Follicles in pairs, usually unequal, rarely equal, $8-8.5 \mathrm{~cm}$ long, straight, erect, tapering at both ends, blunt at tips, glabrous. Seeds 6.5-7 × 1.5$2 \mathrm{~mm}$, narrowly elliptic, brown; wings yellowish, comose; coma 1.8-2.8 cm long, silky white.

Flowering \& fruiting: September-March.

Distribution: Endemic to Tamil Nadu, India.

Specimens examined: INDIA, Tamil Nadu, Naraikkadu (grown in garden), 30.07.2014, S.S. Kambale \& K. Ravikumar SSK 254; Ibid., 08.08.2014, S.S. Kambale \& K. Ravikumar SSK 255; Ibid., 24.08.2014, S.S. Kambale \& K. Ravikumar SSK 293 (SUK!).

Conservation status: So far only reported from the type locality where the area of occupancy is less than $10 \mathrm{sq}$. $\mathrm{km}$. The fragmented populations observed contain a few undivided which are facing severe threat due to habitat destruction. Hence the status is assessed as Critically Endangered [CR: B2 a, b (iii, iv, v)].

Notes: Ceropegia ravikumariana resembles both varieties of C. candelabrum. However, it is distinguished from the latter by its ellipticlanceolate leaves, 2-flowered cymes and gradually dilated corolla tube. 

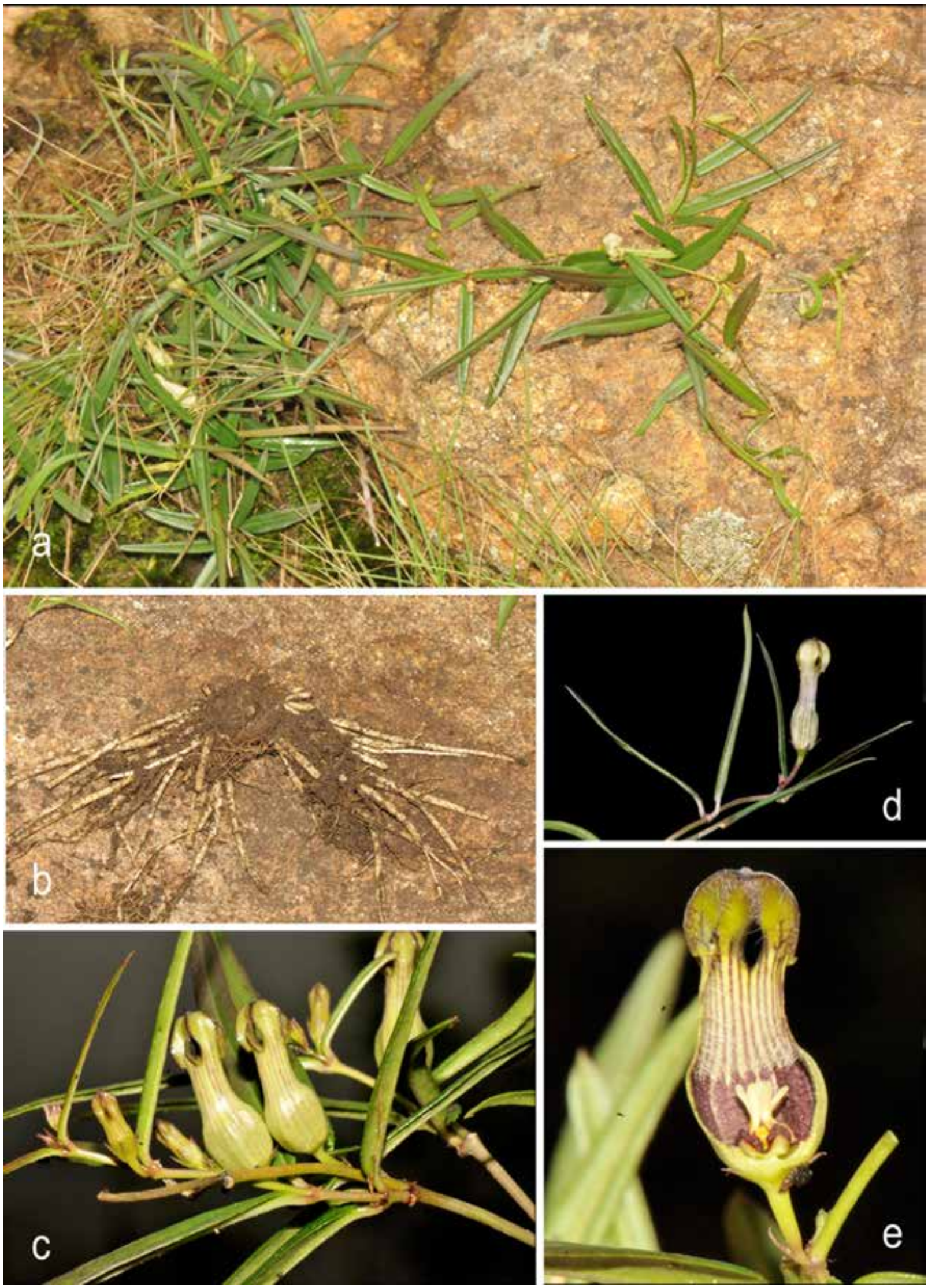

Fig. 7. Ceropegia ravikumariana Kambale \& Gnanasek.: a. Habit; b. Tuber; c. Inflorescence; d. Flower; e. Flower L.S. 


\section{Ceropegia sect. Chionopegia}

Ceropegia sect. Chionopegia H. Huber, Mem. Soc. Brot. 12: 26. 1957; Bruyns, S. African J. Bot. 112: 428. 2017. Type: Ceropegia hookeri C.B. Clarke ex Hook.f.

sect. Hylopegia H. Huber, Mem. Soc. Brot. 12: 27. 1957. Type: Ceropegia lucida Wall.

sect. Oreopegia H. Huber, Mem. Soc. Brot. 12: 26.1957. Type: Ceropegia wallichii Wight

sect. Sinopegia H. Huber, Mem. Soc. Brot. 12: 27. 1957. Type: Ceropegia monticola W.W. Sm.

ser. Macranthae H. Huber, Mem. Soc. Brot. 12: 26. 1957. Type: Ceropegia macrantha Wight

ser. Monticolae H. Huber, Mem. Soc. Brot. 12: 27. 1957. Type: Ceropegia monticola W.W. Sm.

ser. Wallichianae H. Huber, Mem. Soc. Brot. 12: 26. 1957. Type: Ceropegia wallichii Wight

Erect herbs to climbers with fleshy roots. Lamina ovate to linear, petiolate, and glabrous to pubescent. Cymes 1-5-flowered.

Distribution: Twenty two species in the world; Himalaya of Pakistan and India eastwards to China. In India there are nine taxa (Table 1).

\section{Key to the species of sect. Chionopegia}

1. Corolla lobes shorter than tube. 2

1. Corolla lobes equal to tube 7

2. Corolla tube hairy within .3

2. Corolla tube glabrous within .5

3. Corolla lobes glabrous C. pubescens

3. Corolla lobes hairy . .4

4. Plants erect C. wallichii

4. Plants climbing C. longifolia

5. Corolla lobes glabrous. .6

5. Corolla lobes hairy C. kachinensis

6. Leaves ciliate along margins. C. hookeri

6. Leaves glabrous along margins ....... C. angustifolia 7. Corolla lobes hairy outside... C. lucida

7. Corolla lobes glabrous outside. C. macrantha

Ceropegia angustifolia Wight, Contr. Bot. India 31. 1834; Hook.f., Fl. Brit. India 4: 72.1883; P.C. Kanjilal et al., Fl. Assam 3: 308. 1939; Ansari, Fasc.
Fl. India 16: 8. 1984; M.P. Nayar \& Sastry, Red Data Book Indian Pl. 2: 39. 1988; A.P. Jagtap \& N.P. Singh, Fasc. Fl. India 24: 215. 1999; Karthik. et al., Fl. Pl. India 1: 160. 2009. Lectotype (designated by Kambale \& Yadav, 2014): BANGLADESH, Silhet, s.d., N. Wallich Wall. Asclep. n. 13 [K000894290 image!; syntype E00179555, K000894289 image!].

C. longifolia auct. H. Huber, Mem. Soc. Brot. 12: 41. 1957; F. Albers \& Meve, Ill. Handb. Succ. Pl. Asclepiadaceae 63. 2001; G.S. Giri et al., Mat. Fl. Arunachal Pradesh 2: 164. 2008, non Wallich, 1830.

Fig. 8

Perennial, twining to creeping herbs. Stem c. $1 \mathrm{~m}$ long. Lamina 3.5-15 × 1-2.9 cm, elliptic-lanceolate, acute at apex, narrow at base, minutely hairy above, glabrous beneath; petioles 1-1.3 cm long, slender. Cymes umbellate, 5-6-flowered, extraaxillary; flowers dark green to yellowish-brown, brownish at apex; peduncles 1-5.5 cm long, hairy in a row; pedicels $5-15 \mathrm{~mm}$ long, terete, glabrous. Sepals 5-6 mm long, linear, glabrous. Corolla 1.7$4 \mathrm{~cm}$ long; tube 1-2.9 cm long, dilated in lower $1 / 2-1 / 3^{\text {rd }}$ part, sub-cylindrical, funnel-shaped at hairy throat; lobes $0.7-1.1 \mathrm{~cm}$ long, broadly or elliptic-oblong, connate at tip, glabrous, blotched with green. Corona bi-seriate; outer and inner equal; outer of 5 shortly bifid lobes, ciliate along margins and within; inner of 5 erect lobes, linear sub-spathulate lobes. Follicles c. $13.5 \mathrm{~cm}$ long.

Flowering: July-September.

Habitat: Grows amidst grasses.

Distribution: Northeast India, Bangladesh and Nepal. Specimens examined: INDIA, Arunachal Pradesh, Tirap F.D., NEFA, Laju to Raho, 25.08.1958, G. Panigrahi 14748 (ASSAM!). Meghalaya, East Khasi Hills, Bholaganj, 21.08.1935, G.K. Deka 13195; Jarain, 17.08.1968, N.P. Balakrishnan 46162; Khasia Hills, 26.08.1885, C.B. Clarke 400200B (CAL!); Mawrloo forest, 13.09.1931, P.C. Kanjilal 9351; Khasi \& Jaintia Hills, 09.08.1944, G.K. Deka 21882; Ibid., s.d., Gustav Mann 107; Pongtung, 22.08.1938, G.K. Deka 18741; Shillong, Kencha track, 25.08.1966, s.d., Sangama 36898 (ASSAM!).

Mizoram, Lushai Hills district, Mizo hills, 13.08.1965, R. Dutta 34015; Ibid., s.d., S.L. Abbar 102648 (ASSAM!). Nagaland, Kohima, Naga Hills, 27.08.1937, G.K. Deka 16164 (ASSAM!). 


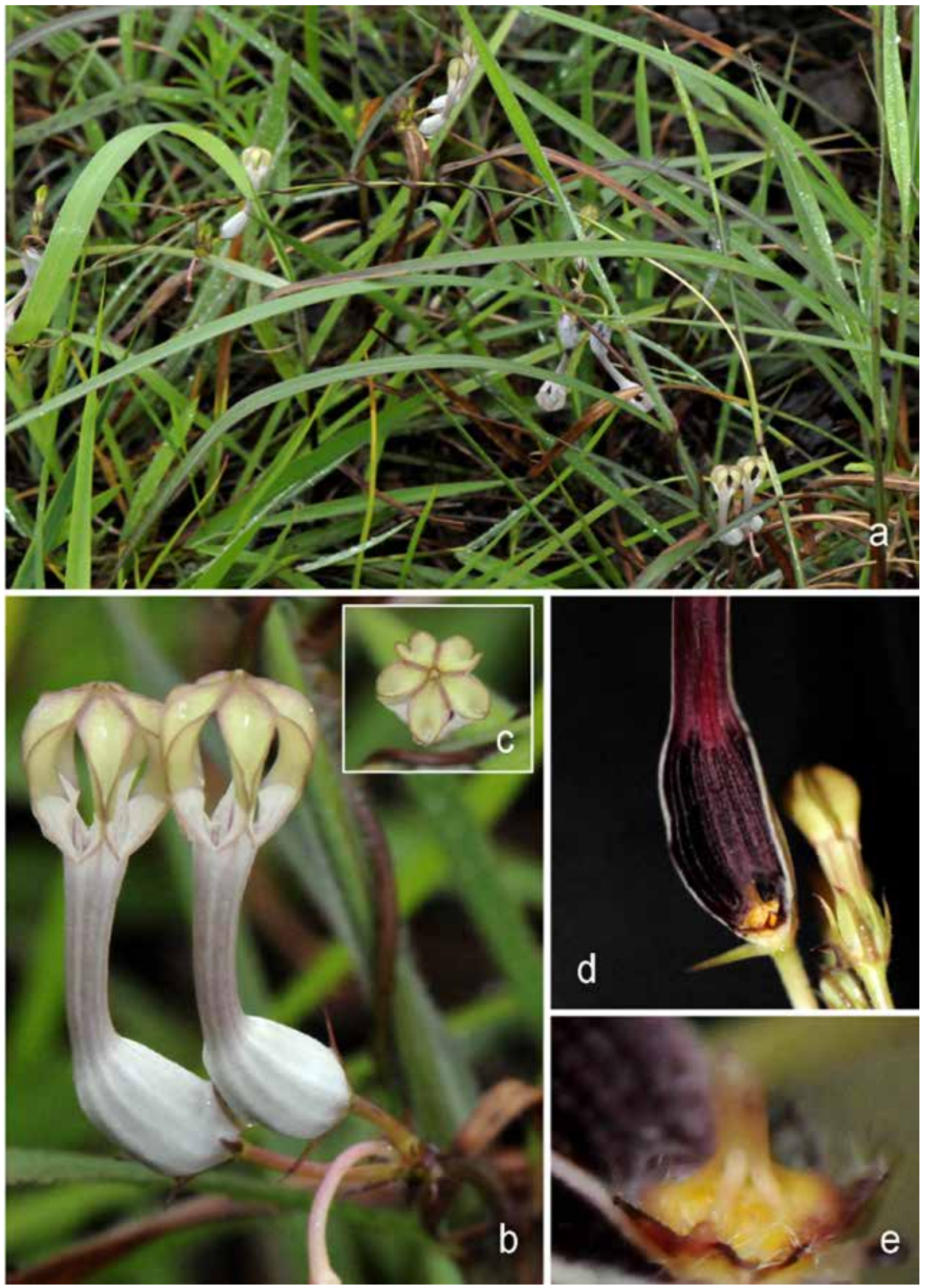

Fig. 8. Ceropegia angustifolia Wight: a. Habit; b. Inflorecence; c. Corolla lobes-top view; d. Flower L.S.; e. Corona-lateral view (Photos Dr. K. Prasad). 
Conservation status: This species was reported from four different states in NE India. However no recent collection is available in any Indian herbaria we consulted. Hence, we treat the status as Data Deficient (DD) due to inadequate information.

Notes: Huber (1957) considered C. angustifolia and C. longifolia Wall. as conspecific. While Ansari (1984) treated them as distinct species. After thorough scrutiny of the type specimens and live materials of both the taxa, authors prefer to keep them as distinct. Ceropegia angustifolia can be distinguished from the latter by its white, glabrous corolla. It is also similar to C. rollae Hemadri but differs from its climbing habit and deeply bifid outer corona.

Ceropegia hookeri C.B. Clarke ex Hook.f., Fl. Brit. India. 4: 73. 1883; H. Huber, Mem. Soc. Broter. 12: 47. 1957; Ansari, Fasc. Fl. India 16: 17. 1984; M.P. Nayar \& Sastry, Red Data Book Indian Pl. 2: 42. 1988; A.P. Jagtap \& N.P. Singh, Fasc. Fl. India 24: 224. 1999; F. Albers \& Meve, Ill. Handb. Succ. Pl. Asclepiadaceae 82. 2002; Chettri et al., Check List 5(3): 695. 2009. C. hookeri C.B. Clarke ex Hook.f. var. hookeri Karthik. et al., Fl. Pl. India 1: 162. 2009. Lectotype (Step I designated by Huber, 1957; Step II designated here): INDIA, Sikkim, Lachen, J.D. Hooker Ceropegia 23 (K000857824 image!).

Fig. 9

Perennial twining herbs. Rootstock with globose tuber. Stem slender, hirsute. Lamina 4-5.7 × 1-2 $\mathrm{cm}$, ovate-lanceolate to elliptic-lanceolate, acute at apex, rounded at base, hirsute above, ciliate along

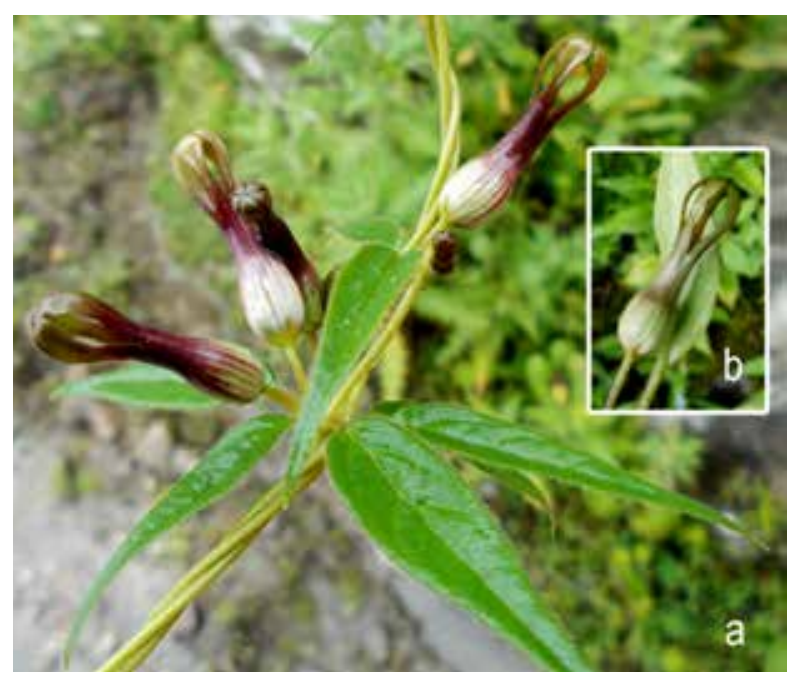

Fig. 9. Ceropegia hookeri C.B. Clarke ex Hook.f.: a. Habit; b. Flower (Photos Dr. D. Maity). margins and nerves beneath, glabrous otherwise; petioles 3-8 mm long, slender, glabrous; Cymes 1-3-flowered, extra-axillary; peduncles 5-7 $\mathrm{mm}$ long, pilose; pedicels $3-5 \mathrm{~mm}$ long, terete, glabrous. Sepals 3-4.5 × 0.6-1 mm, linearlanceolate, glabrous. Corolla c. $2.2 \mathrm{~cm}$ long, dark purple-faint green with purple tinge; tube c. 1.5 $\mathrm{cm}$ long, gradually dilated at base, cylindrical at middle, hairy within; lobes 7-8 mm long, linear, glabrous, connate at the tip forming an ovoid cage. Corona bi-seriate; outer of 5 entire, ciliate lobes; inner of 5 erect, linear lobes. Follicles $c .5 \mathrm{~cm}$ long. Flowering \& fruiting: June-September.

Habitat: Found growing in grasslands, along slopes.

Distribution: India (Sikkim), Nepal and Tibet.

Conservation status: Nayar and Satry (1987) assessed it as Endangered. However we could not come across any live specimens or herbarium specimens during this study. The information given is based on few previous reports.

Notes: Hooker (1883) published C. hookeri based on the collections from Lachen, in Sikkim. Huber (1957) cited specimen at K as the holotype, which can be considered as first step lectotypification. But at K, three specimens (K000857823, K000857824 and K000857825) are available. Sheet K000857824 is selected here as second step lectotype following Art. 9.17 of Shenzhen Code (Turland et al., 2018).

Ceropegia kachinensis Prain, J. Asiat. Soc. Bengal, Pt. 2, Nat. Hist. 69(2): 170. 1900; H. Huber, Mem. Soc. Brot. 12: 50. 1957; Ansari, Fasc. Fl. India 16: 19. 1984; A.P. Jagtap \& N.P. Singh, Fasc. Fl. India 24: 227. 1999; Karthik. et al., Fl. Pl. India 1: 162. 2009. Lectotype (designated by Singh, 2015): MYANMAR, Upper Burma, Kachin Hills, Myitkyina, 11.1897, Shaik Mokim s.n. (K000857821 image!; isolectotype CAL0000018016!, DD!, M0175137 image!, Z000001595 image!).

C. farrokhii McCann, J. Bombay Nat. Hist. Soc. 45: 210. 1945. Type: MYANMAR, Upper Burma, Kachin Hills, Myitkyina (grown in Royal Botanic Garden, Kolkata), 11.1902, Shaik Mokim s.n. (CAL0000018015!; iso CAL0000018014!).

Perennial, extensively twining herbs. Rootstock with globose tuber. Lamina $2.2-12 \times 0.4-5 \mathrm{~cm}$, ovate-lanceolate or elliptic, rounded at base, acute at apex, glabrous on both surfaces, membranous, deep 
green above, pale beneath; petioles $0.6-2 \mathrm{~cm}$ long, glabrous. Cymes 5-12-flowered, extra-axillary; peduncles $2-2.5 \mathrm{~cm}$ long, glabrous; pedicels $1.2-$ $1.5 \mathrm{~cm}$ long, slender, glabrous. Sepals c. 2-5.2 mm long, subulate, glabrous, with pinkish tinge at the tip. Corolla 3-3.2 cm long; tube 1.5-2.5 cm long, dilated at the base, slightly curved, narrow at middle, funnel-shaped at throat, externally pale yellowish green with striated purple on lower $2 / 3^{\text {rd }}$ part, faint purple spots on upper $1 / 3^{\text {rd }}$ part, purple within with deep purple dark windows at lower $2 / 3^{\text {rd }}$ part, bright green at lower and pale yellow in upper-half; lobes $c$. $5 \mathrm{~mm}$ long, oblong, shorter than tube, ciliate along margins with purple hairs at upper $1 / 3^{\text {rd }}$ part. Corona bi-seriate; outer of 5 dentate, ciliate reddish-purple lobes; inner of 5 erect linear-clavate lobes. Follicles 8-10 cm long, variously blotted with pale purple streaks. Seeds c. $10 \times 4 \mathrm{~mm}$, ovate, compressed.

Flowering \& fruiting: October-November.

Habitat: Found growing in shady places in forests.

Distribution: India (Sikkim), Bangladesh and Myanmar.

Specimen examined: INDIA, Sikkim, s.loc., s.d., King s.n. (CAL!).

Conservation status: This species has not been collected after type collection and is poorly represented in Indian herbaria. In the present study authors could trace only four specimens two at $\mathrm{CAL}$, one at $\mathrm{K}$ and one at $\mathrm{Z}$. It is here assessed as Data Deficient (DD).

Notes: It is close to C. thwaitesii but differs in having smaller flowers. Prain (1900) described C. kachinensis based on Shaikh Mokim's specimens. In the protologue, Prain mentioned the locality as Kachin Hills, Myitkyina. Huber (1957) and Ansari (1984) cited "Typus: CAL (Holotypus)". Since there are 4 specimens at CAL each with a different date, neither the designation of type by Huber or Ansari can be considered a valid lectotypification. However, Singh (2015) selected a specimen from Kew (K000857821) as the lectotype as it agrees with the protologue.

Ceropegia longifolia Wall., Pl. Asiat. Rar. 1: 56, t. 73. 1830; Hook.f., Fl. Brit. India 4: 72. 1883; H.J. Chowdhery in H.J. Chowdhery \& Wadhwa, Fl. Himachal Pradesh 461. 1984; Ansari, Fasc. Fl. India 16: 20. 1984; A.P. Jagtap \& N.P. Singh, Fasc. Fl. India 24: 228. 1999; F. Albers \& Meve,
Ill. Handb. Succ. P1. Asclepiadaceae 87. 2002; G.S. Giri et al., Mat. Fl. Arunachal Pradesh 2: 164. 2008; Karthik. et al., Fl. Pl. India 1: 162. 2009. Lectotype (designated by Huber, 1957): NEPAL, s.loc., s.d., N. Wallich 11 (K001129051 image!; syntype E00288765 image!).

C. lanceolata Wight \& Arn., Contr. Bot. India 31. 1834. Lectotype (designated by Singh, 2015: 158): NEPAL, s.loc., s.d., N. Wallich, Ascl. n. 12 (K000857830 image!).

C. borii Raizada, Indian Forest Rec., Bot. 3: 121. 1941; Babu, Herbac. Fl. Dehra Dun 305. 1977. Syntype: INDIA, Uttarakhand, Mohand pass, s.d., Raizada \& Champion s.n. (DD).

Perennial twining herbs with fascicled roots. Stem slender, puberulous, branched. Lamina 5.7-20.3 × 0.4-3.8 cm, elliptic, lanceolate or linear, acute at apex, rounded at base, puberulous, ciliate along margins, glabrous beneath; petioles 5-8 $\mathrm{mm}$ long, slender. Cymes umbellate, 5-12-flowered, extra-axillary; peduncles 1.8-4 $\mathrm{cm}$ long, puberulous. Flowers whitish-green to yellowish-green; pedicels $0.5-1.2 \mathrm{~cm}$ long, terete, puberulous. Sepals c. $1.2 \mathrm{~cm}$ long, linear-subulate, puberulous. Corolla $1.6-3.8 \mathrm{~cm}$ long; tube $0.5-$ $2.5 \mathrm{~cm}$ long, curved, dilated at base, funnelshaped at throat, hairy within at dilated part; lobes 0.5-1 cm long, elliptic-oblong to broadly ovate, reflexed, connate at the tip forming ovoid cage, hairy within, greenish-yellow, blotched purple. Corona bi-seriate; outer of 5 deeply bifid-deltoid lobes, ciliate along margins and within, yellow at base, purple at margins; inner of 5 erect, linear sub-spathulate lobes. Follicles $12.5-15.2 \mathrm{~cm}$ long, slender. Seeds c. $8 \mathrm{~mm}$ long, ovate, marginate, comose; coma silky white.

\section{Key to the subspecies}

1. Corolla lobes broadly ovate, c. $1 \mathrm{~cm}$ long subsp. longifolia

1. Corolla lobes oblong to linear, rarely ovate, $c$. $2.5 \mathrm{~cm}$ long subsp. sinensis

\section{Ceropegia longifolia Wall. subsp. longifolia}

Fig. 10 (a \&b)

Flowering \& fruiting: June-September.

Habitat: In crevices of rocky cliffs, in shady places and along slopes in grasslands. 
Distribution: India (Arunachal Pradesh, Meghalaya, Nagaland, Sikkim, Uttar Pradesh, West Bengal), Bangladesh and Nepal.

Specimens examined: INDIA, Meghalaya, Shillong, 18.08.1886, C.B.Clarke 44566 (CAL!).Sikkim, s.loc., 1881, King s.n. (CAL!). Uttarakhand, Dehradun district, 08.1897, P.W. Mackinnon s.n:; Mussoorie, 27.08.1898, s.coll. s.n. (CAL!). West Bengal, s.d., Darjeeling district, Kalimpong, J. Ripley 1261128 (CAL!).

Conservation status: It is a widely distributed species but the population has decreased in last few years. It is poorly represented in India as well as overseas herbaria. This indicates the rarity of the species. As the available information is inadequate, we treat the status as Data Deficient (DD).

Ceropegia longifolia subsp. sinensis H. Huber, Mem. Soc. Brot. 12: 42. 1957. Type: CHINA, Western Szechuan, 06.1908, E.H. Wilson 2313 (holo GH image!).

C. mizoramensis Ram. Kumar \& S. Sharma, Taiwania 63(2): 164. 2018. syn. nov. Type: INDIA, Mizoram, Champhai, Murlen National Park, $c$. 1100 m, 18.09.2014, R. Kumar \& S. Sharma 131485 (holo ASSAM!; iso CAL!).

C. murlensis Ram. Kumar \& S. Sharma, Taiwania 63(2): 166. 2018. syn. nov. Type: INDIA, Mizoram, Champhai, Murlen National Park, c. 1100 m, 18.09.2014, R. Kumar \& S. Sharma 131486 (holo ASSAM!; iso CAL!).

Lamina 6-9 × 1-2 cm, linear to elliptic-lanceolate, acute at apex, narrow at base, glabrous above pubescent beneath; petioles c. $1.2 \mathrm{~cm}$ long. Cymes umbellate, 2-12-flowered, extra-axillary; peduncles c. $2.5 \mathrm{~cm}$ long. Flowers whitish-green to yellowish-green; pedicels $0.4-1.5 \mathrm{~cm}$ long, terete, puberulous. Corolla c. $5 \mathrm{~cm}$ long; tube c. $2.5 \mathrm{~cm}$ long, straight to slightly curved, dilated at base, funnel-shaped at throat, ring of hairs at dilated part; lobes c. $2.5 \mathrm{~cm}$ long, elliptic-oblong to broadly ovate, reflexed half to completely, connate at the tip forming ovoid to linear cage, hairy within, hairs are translucent, variously colored, red to greenish in the upper-half, yellowish green to purple veined in lower half, .

Flowering \& fruiting: August-October.

Distribution: India (Mizoram) and China.
Notes: C. longifolia subsp. sinensis differs from typical variety in having oblong to linear corolla lobes. Huber (1957) recognized subsp. sinensis based on the specimens collected by Wilson from China. Ansari (1984) reported this taxon in India based on the specimens collected by G.K. Deka (ASSAM). On critical examination of the types and protologue, it is concluded that this specimen does not match with subsp. sinensis. Kumar et al. (2018) recently described two new species viz. C. mizoramensis Ram.Kumar \& S. Sharma and C. murlensis Ram.Kumar \& S. Sharma from north east India. The characters used by the authors to erect these taxa are found to intergrade with the circumscription of C. longifolia Wall. subsp. sinensis, hence both are reduced to the synonymy of C. longifolia var. sinensis.

Ceropegia lucida Wall., Pl. Asiat. Rar. 2: 33, t. 139. 1831; Hook.f., Fl. Brit. India 4: 73. 1883; P.C. Kanjilal et al., Fl. Assam 3: 309. 1939; H. Huber, Mem. Soc. Brot. 12: 54. 1957; Ansari, Fasc. Fl. India 16: 22. 1984; M.P. Nayar \& Sastry, Red Data Book Indian Pl. 2: 44. 1988; A.P. Jagtap \& N.P. Singh, Fasc. Fl. India 24: 229. 1999; Karthik. et al., Fl. Pl. India 1: 162. 2009; Khandal et al., Curr. Sci. 113(11): 2077. 2017. Lectotype (Step I designated by Huber, 1957; Step II designated here): BANGLADESH, Sylhet, (Sillet), s.d., N. Wallich Asclep. 2 (K000857816 image!).

Fig. 10 (C)

Perennial twining herbs. Stem sparsely hairy to glabrous. Lamina 7-9 cm long, elliptic-lanceolate to oblong-lanceolate, acute-acuminate at apex, narrowed at base, slightly fleshy, glabrous, ciliolate along margins, dark green above, pale below; petioles $0.8-1 \mathrm{~cm}$ long, slender, pilose. Cymes umbellate, 5-6-flowered, extra-axillary; peduncles $c .2 \mathrm{~cm}$ long, fleshy, puberulous. Flowers yellowish-green with purple spots; pedicels 1-1.8 $\mathrm{cm}$ long, terete, glabrous. Sepals linear-subulate, glabrous. Corolla 1.8-2.7 cm long; tube 1.2-2.5 $\mathrm{cm}$ long, cylindrical throughout, hardly dilated at base, cylindrical, funnel-shaped at throat, with a ring of hairs at the throat of dilated part; lobes 0.7$2.5 \mathrm{~cm}$ long, broadly or elliptic-oblong, spathulate, connate at the tip, hairy within at upper-half, greenish yellow blotched with purple. Corona biseriate; outer of 5 shortly bifid-deltoid lobes, ciliate along margins; inner of 5 erecto-divergent, linearclavate, lobes. Fruits not observed. 

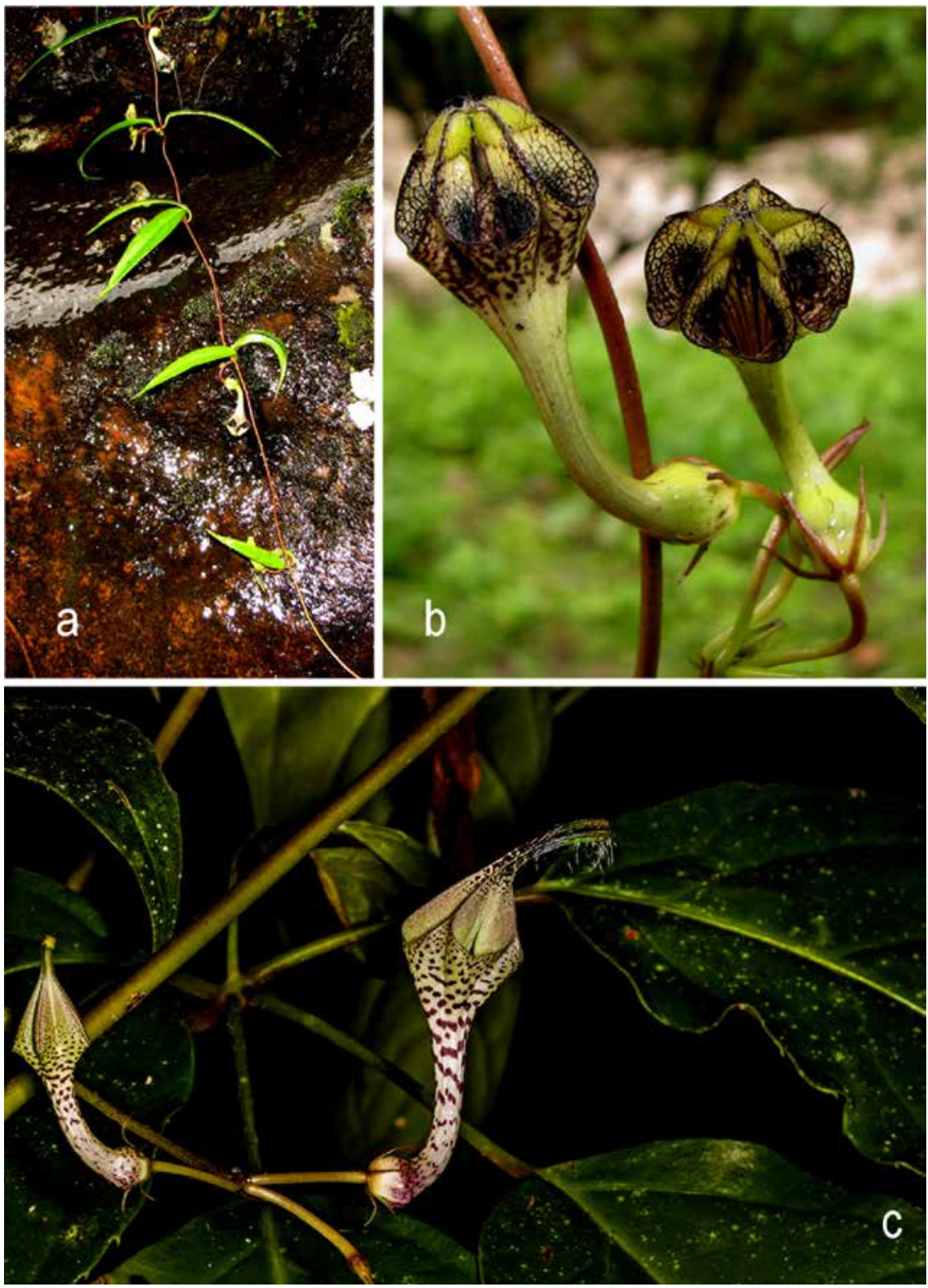

Fig. 10. Ceropegia Iongifolia Wall.: a. Habit; b. Flowers (Photos Dr. Umeshkumar Tiwari). c. Ceropegia lucida Wall. (Photos Mr. Dharmendra Khandal). 
Flowering: September-November.

Habitat: Found growing along streams in forests on rocky substratum.

Distribution: India (Arunachal Pradesh, Sikkim), Bangladesh, China, Hong Kong, Malaysia, Myanmar and Thailand (SIAM).

Specimen examined: INDIA, Sikkim, Sikkim Himalaya, 1874, G. King s.n. (CAL!).

Conservation status: Nayar and Sastry (1987) assessed C. Lucida as Endangered or possibly Extinct. However, Khandal et al. (2018) reported it from Arunachal Pradesh, which is a clear indication of its existence in the wild. However, the surviving population comprises less than 10 mature individuals and is therefore assessed as Critically Endangered [CR: B1 a, b (iii, iv, v)].

Notes: Wallich (1831) described C. Iucida from Sylhet in Bangladesh. Without mentioning any specimen in the protologue. Huber (1957) cited "Typus: Wallich (Sillet) in Herb. K (Holotypus), P”. As there are two sheets at K (K000857816 \& K001129042), Huber's selection can be considered as the first step of lectotypification. To narrow down the choice, K000857816 is designated here as the second step lectotype following Art. 9.17 of Shenzhen Code (Turland et al., 2018).

Ceropegia macrantha Wight, Contr. Bot. India 31. 1834; Hook.f., Fl. Brit. India 4: 74. 1883; Collett, Fl. Siml. 320. 1902; Prain, Bengal Pl. 2: 701. 1903; H. Huber, Mem. Soc. Brot. 12: 45. 1957; Ansari, Fasc. Fl. India 16: 23. 1984; H.J. Chowdhery in H.J. Chowdhery \& Wadhwa, Fl. Himachal Pradesh 462. 1984; A.P. Jagtap \& N.P. Singh, Fasc. Fl. India 24: 230. 1999; F. Albers \& Meve, Ill. Handb. Succ. Pl. Asclepiadaceae 88. 2002; Karthik. et al., Fl. Pl. India 1: 162. 2009. Lectotype (Step I designated by Huber, 1957; Step II designated by Singh, 2015): INDIA, Uttar Pradesh, Kumaon, Khurie Pass, s.d., J.F. Royle s.n. (K000857826 image!).

Ceropegia raizadiana Babu, Herbac. Fl. Dehra Dun 305. 1977. Type: INDIA, Uttarakhand, Dehradun, Bindal, s.d., C.R. Babu 32215 (holo CAL, apparently none preserved; iso $\mathrm{BSD}$ !).

Fig. 11

Perennial twining herbs. Stem climbing, glabrous, hairy at nodes. Lamina $4.5-14 \times 2-7 \mathrm{~cm}$, ovatelanceolate, acute-acuminate at apex, rounded at base, sparsely hairy above, ciliolate along margins, glabrous beneath; petioles 1.2-2.4 cm long, slender, channeled above, puberulous. Cymes subumbellate, 4-5-flowered, extra-axillary; peduncles 1.2-2.7 cm long, hairy in 1 or 2 rows. Flowers yellowish blotched with pink throughout; pedicels c. $8 \mathrm{~mm}$ long, terete, glabrous. Sepals $6-7 \mathrm{~mm}$ long, linear-subulate, glabrous. Corolla 2.5-7.6 cm long; tube 1.8-3.2 cm long, abruptly dilated at base, cylindrical at middle, funnel-shaped at throat, ciliolate throughout, with a ring of hairs at the mouth within, glabrous otherwise, deep pink within, dilated part blotched pink inside; lobes 2.4$2.8 \mathrm{~cm}$ long, linear to elliptic-lanceolate, connate at the tip forming ovoid cage, glabrous outside, hairy within at upper-half of linear part, yellow at lower and dull green at upper-half. Corona bi-seriate; outer of 5 deeply bifid lobes, densely hairy, pink; inner of 5 erect lobes, linear. Follicles $10.5-12 \mathrm{~cm}$ long.

Flowering \& fruiting: April-August.

Habitat: Usually found growing along slopes in the forest, in well drained soil.

Distribution: India (Jammu \& Kashmir, Himachal Pradesh, Sikkim, Uttar Pradesh, West Bengal), Bhutan, Nepal and Pakistan.

Specimens examined: INDIA, Arunachal Pradesh, West Kameng, Aka hills, 04.1937, N.L. Bor 15756 (ASSAM!). Assam, Nowgong district, Kholahat reserve forest, 01.09.1964, N.P. Balakrishnan 39589 (ASSAM!). Nagaland, Naga Hills, 06.1936, N.L. Bor 20763 (ASSAM!).

Conservation status: During the present study the plant could not be collected. Due to insufficient information the status is attributed as Data Deficient (DD) for want of more study.

Notes: Ceropegia macrantha apparently looks like C. vincifolia but differs in lacking horizontal light window. It is also allied to C. elegans in having ring of hairs at the dilated part of the corolla tube within but can be distinguished by its shorter and densely hairy outer corona.

Ceropegia pubescens Wall., Pl. Asiat. Rar. 2: 81, t. 187. 1831; Hook.f., Fl. Brit. Ind. 4: 73. 1883; P.C. Kanjilal et al., Fl. Assam 3: 308. 1939; H. Huber, Mem. Soc. Brot. 12: 47. 1957; Ansari, Fasc. Fl. India 16: 28. 1984; A.P. Jagtap \& N.P. Singh, Fasc. Fl. India 24: 236. 1999; F. Albers \& Meve, Ill. Handb. 

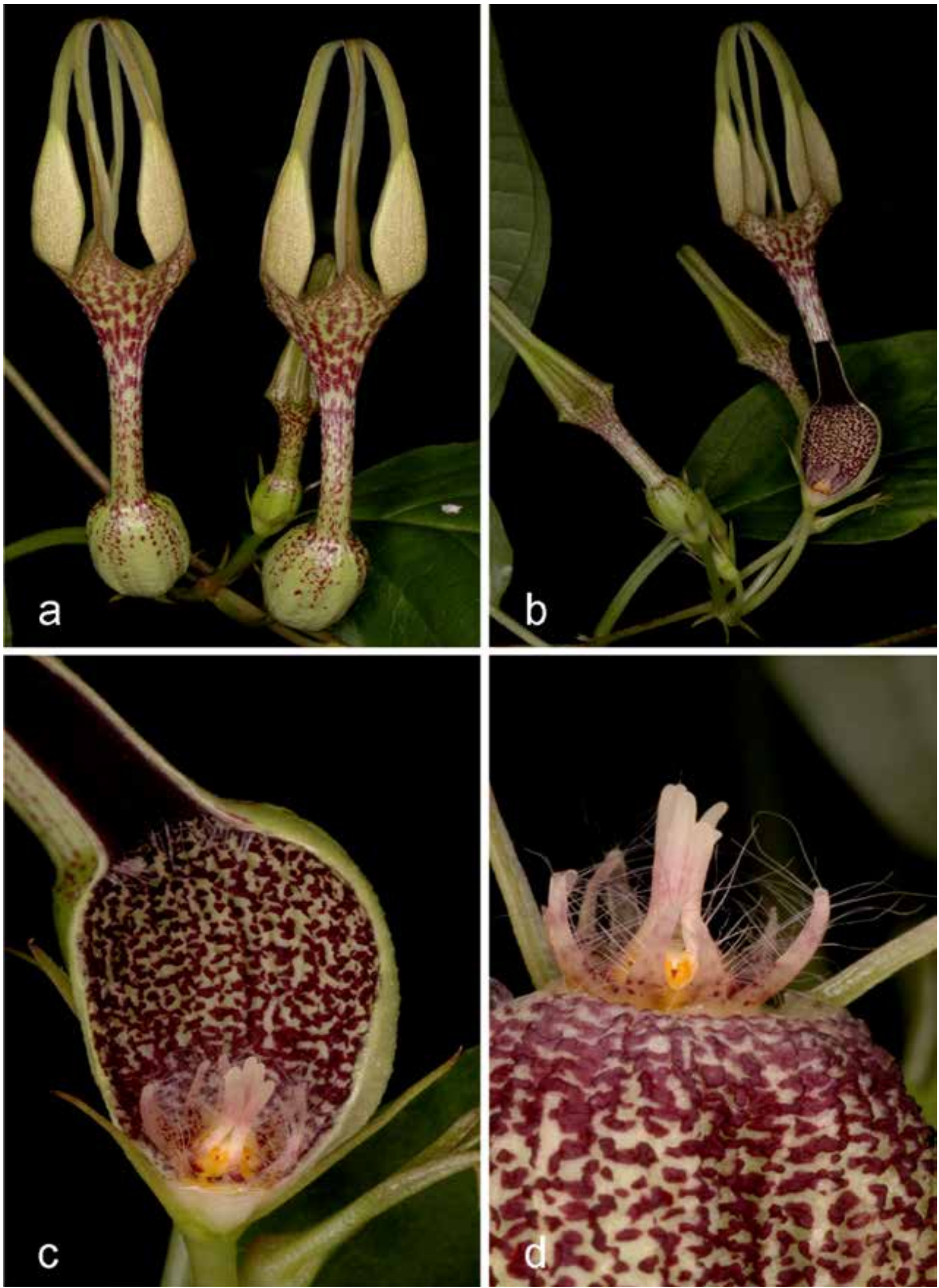

Fig. 11. Ceropegia macrantha Wight a. Habit with inflorescence; b. Flower L.S.; c. Flower L.S.-close-up; d. Corona-lateral view (Photos Navendu Page). 
Succ. Pl. Asclepiadaceae 95. 2002; Karthik. et al., Fl. Pl. India 1: 163. 2009. Lectotype (designated by Singh, 2015): NEPAL, Chandaghiry, 1821, N. Wallich Asclep. n. 10. (K000014465 image!; isolectotype K000014463 image!).

Fig. 12

Perennial twining herbs. Stem glabrous. Lamina 6.5-10.5 × 2.8-6.0 cm, ovate-lanceolate, acute at apex, rounded at base (rarely cordate), sparsely hairy above, glabrous beneath; petioles $2-2.8 \mathrm{~cm}$ long, terete to channeled, pilose. Cymes umbellate, many-flowered, extra-axillary; peduncles 3.4-5.8 $\mathrm{cm}$ long, terete, hairy in row; pedicels $c .1 .5-1.8$ $\mathrm{cm}$ long, terete, glabrous. Sepals 3-4 mm long, acute, glabrous. Corolla 5.4-6.5 cm long; tube $1.5-3.3 \mathrm{~cm}$ long, gradually dilated towards base, cylindrical otherwise, hairy within with purple striations, grey to purple outside; lobes 1.8-3 $\mathrm{cm}$ long, ovate-lanceolate at lower half, linear at upper-half, connate at tips, twisted at maturity, glabrous, purple outside, yellow within at lower half, dull green to pale purple at upper-half. Corona bi-seriate; outer of 5 lobes, glabrous or hairy; inner linear, erect. Fruits not observed.

Flowering \& fruiting: June-October.

Habitat: Grows along ghat roadsides adjoining moist forests.

Distribution: India (Meghalaya, Nagaland, Sikkim, West Bengal), Bhutan, China (Yunnan) Nepal and Tibet.

Specimens examined: INDIA, Assam, Darjeeling district, Darjeeling, 17.09.2013, P.R. Kshirsagar \& N.B. Gaikwad SSK 88 (SUK!); Pankhabai, 08.1880, J.S. Gamble 8319 (CAL!). Sikkim, Eastern Himalaya, Berch Hill, 05.07.1956, D. Chatterjee 42 (CAL!); Sikkim Himalaya, 11.09.1869, C.B. Clarke 9037; Ibid., 30.08.1877, G. King 4047; Ibid., 22.09.1877, G. King 4910 (CAL!).

Notes: Ceropegia pubescens is closer to C.candelabrum var. biflora (L.) Ansari but differs in its pubescent leaves ( $v s$. glabrous leaves) and glabrous corolla lobes (vs. hirsute lobes).

Ceropegia wallichii Wight, Contr. Bot. India 32. 1834; Royle, Ill. Bot. Himal. Mts. t. 66. 1839; Decne. in DC., Prodr. 8: 644. 1844; Hook.f., Fl. Brit. India 4: 67. 1883; H. Huber, Mem. Soc. Brot. 12: 39. 1957; Ansari, Fasc. Fl. India 16: 32. 1984; A.P. Jagtap \& N.P. Singh, Fasc. Fl. India 24:
241. 1999; F. Albers \& Meve, Ill. Handb. Succ. Pl. Asclepiadaceae 106. 2002; Karthik. et al., Fl. Pl. India 1: 164. 2009. Lectotype (Step I designated by Huber, 1957; Step II designated by Singh, 2015): INDIA, Uttar Pradesh, Kumaon, Lahoolghat, s.d., A.K. Lindsay s.n. (E00179561 image!; isolectotype K000975598 image!).

Fig. 13

Perennial erect herbs. Rootstock with a fascicle of tuberous roots, $9-10 \times 0.5 \mathrm{~cm}$. Stem $13-40 \mathrm{~cm}$ long, robust, hairy above, glabrous otherwise. Leaves subsessile to shortly-petiolate; lamina $1.5-8.5 \times 0.5-3$ $\mathrm{cm}$, ovate to elliptic-lanceolate, acute-acuminate at apex, narrow at base, fulvous throughout; petioles $c$. $1 \mathrm{~cm}$ long. Flowers solitary, extra-axillary; pedicels 5-6 mm long. Sepals 7-8 mm long, pubescent. Corolla 4-5.4 cm long; tube 2.8-4 cm long, gradually dilated towards base, funnel-shaped at throat, hairy within; lobes $1.2-1.5 \mathrm{~cm}$ long, oblong, connate at the tip forming ovoid-cage, densely hairy. Corona bi-seriate; outer of 5-bifid lobes, ciliate along margins; inner of 5 erect, linear lobes. Follicles 17-20.5 cm long, glabrous. Seeds $8-9 \times c .4$ $\mathrm{mm}$, ovate, marginate, brown, comose; coma silky white.

Flowering \& fruiting: May-August.

Distribution: India (Himachal Pradesh, Uttarakhand) and Nepal.

Specimens examined: INDIA, Uttarakhand, Nainital district, Kumaon, towards Kaladungi, 08.06.1985, J.F. Duthie 4196; North West Himalaya; s.d., P.W. Mackinon s.n. (CNH!); s.loc., 04.07.1990, S. Rotra s.n. (CAL!).

Conservation status: It is represented by few sheets at Indian herbaria, moreover it has not been collected during this study. It is assessed here as Data Deficient (DD).

Notes: In the protologue Wight (1834) cited 'Wight in Bot. Mag. tab 3267' which in turn is C. wightii Graham, a glabrous twiner, which is now treated as C. africana R.Br.

\section{Ceropegia sect. Esculentae}

Ceropegia sect. Esculentae Bruyns, S. African J. Bot. 112: 412. 2017. Type: Ceropegia bulbosa Roxb.

Climbing tuberous succulent herbs. Leaves succulent, linear-lanceolate to ovate, petiolate to sub-sessile, glabrous. Cymes c. 13-flowered. 

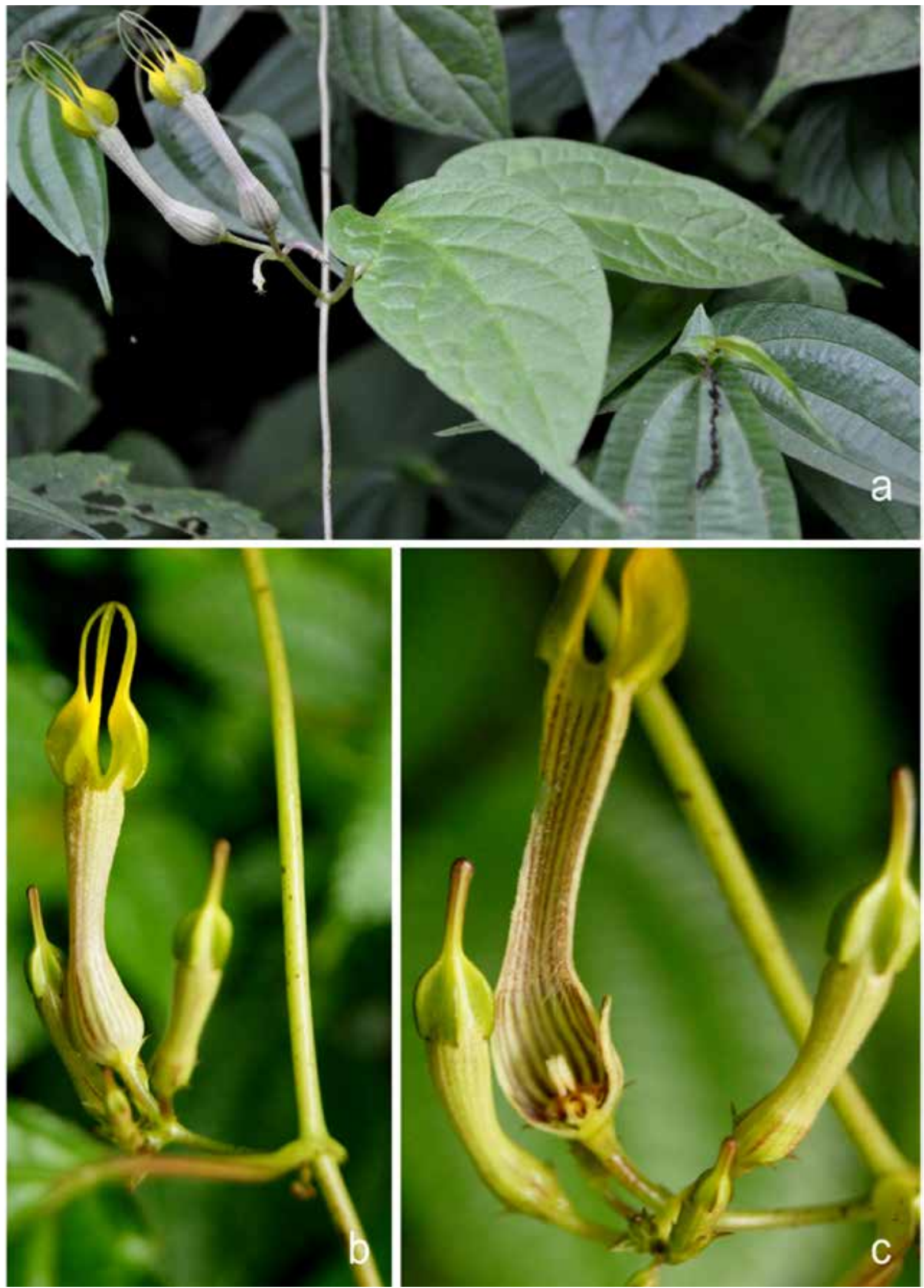

Fig. 12. Ceropegia pubescens Wall.: a. Habit; b. Inflorescence; c. Flower L.S. (Photos Dr. Nikhil Gaikwad). 


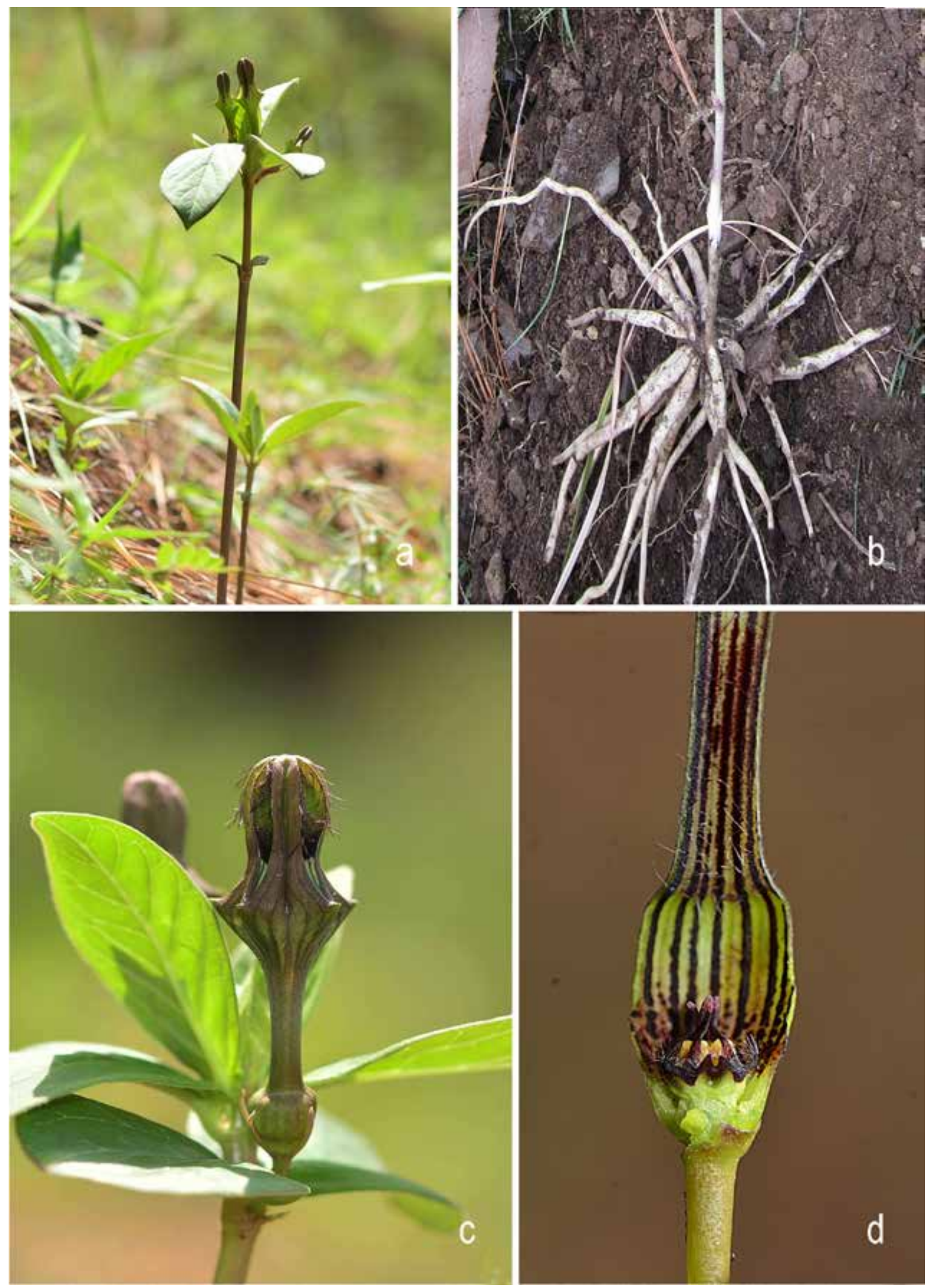

Fig. 13. Ceropegia wallichii Wight: a. Habit; b. Rootstock; c. Flower; d. Flower L.S. (Photos Mr. Sushant More). 
Corolla tube hairy within.

Distribution: Five species in the world; west to northeast Africa, southern Arabian Peninsula, southeast Asia from Pakistan to central and Peninsular India. In India the section is represented by a single species i.e. C. bulbosa Roxb. (Table 1).

Ceropegia bulbosa Roxb., Pl. Coromandel 1: 11. t. 7. 1795; Dalzell \& A. Gibson, Bombay Fl. 153. 1861; Hook.f., Fl. Brit. India 4: 67. 1883; Duthie, Fl. Gangetic Plain 2: 66. 1911; H. Huber, Mem. Soc. Broter. 12: 60. 1957; Gamble, Fl. Madras 4: 857.1957; T. Cooke, Fl. Bombay 2: 240. 1958; Ansari, Fasc. Fl. India 16: 11. 1984; B.D. Sharma et al., Fl. Karnataka, Analysis 165. 1984; Fl. Akola District, Maharashtra 130. 1988; B.V. Shetty \& V. Singh, Fl. Rajasthan 2: 476. 1991. S.D. Deshp. et al., Fl. Mahabaleshwar, Maharashtra 1: 360. 1993; Hanumanthappa in Pull. \& Moulali, Fl. Andhra Pradesh 2: 584. 1997; Naik, Fl. Marathwada 1: 544. 1998; A.P. Jagtap \& N.P. Singh, Fasc. Fl. India 24: 217. 1999; A.P. Jagtap \& Das Das in N.P. Singh et al., Fl. Maharashtra, Dicot. 2: 349. 2001; M.R. Almeida, Fl. Maharashtra 3A: 229. 2001; F. Albers \& Meve, Ill. Handb. Succ. Pl. Asclepiadaceae 70. 2002; T.S. Nayar et al., Fl. Pl. Kerala 84. 2006; Karthik. et al., Fl. Pl. India 2: 160. 2009; Kambale \& S.R. Yadav, Asklepios 115: 29. 2013. Lectotype (designated by Huber, 1957): Roxb. Pl. Coromandel 1: 11, t. 7.

Vernacular names: Daichandi (Hindi), Jolaka, Hallike (Kannada), Galya, Kanvel, Kharpudi, Khartundi, Khapparkadu (Marathi), Commo manda, Settu nimatayalu (Telugu).

Perennial twining herbs. Rootstock tuberous; tubers $c .5 \times 2 \mathrm{~cm}$, sub-globose, depressed, subspherical or oblong, roots fibrous. Stem c. 1.5 $\mathrm{m}$ long, usually single from each tuber, rarely two, terete, glabrous. Lamina 1.2-4.6 × 1.1-4.5 $\mathrm{cm}$, lower orbicular, upper elliptic-oblong or ovate-cordate, acuminate at apex, rounded or sub-cordate to cordate at base, lateral nerves 3-5 pairs, slightly ciliolate along margins, glabrous otherwise; petioles $0.4-1.0 \mathrm{~cm}$ long, channeled above, slightly hairy at apex, glabrous otherwise. Cymes 4-13-flowered, extra-axillary; peduncles $1.2-2 \mathrm{~cm}$ long, terete, glabrous; bracts $1.2-3 \times 0.35$ $\mathrm{mm}$, subulate, glabrous; pedicels c. $5 \mathrm{~mm}$ long, terete, glabrous. Corolla $1.4-2.3 \mathrm{~cm}$ long; tube $1-1.5 \mathrm{~cm}$ long, slightly curved, abruptly dilated at base, funnel-shaped at throat, glabrous outside, hairy within except for dilation part, greyish-green with pink blotches at the dilation and at the base of corolla lobes, faint purple within with striations at dilated part; lobes 4-8 mm long, reflexed on back, connate at tip forming an obovate-ellipsoid cage, linear from triangular base, obtuse, hairy within and along margins, deep purple-green at upperhalf, hairs purple-pink. Corona bi-seriate, stipitate; outer of 5 lobes, equal, c. $3 \mathrm{~mm}$ long, saucer-shaped, glabrous, white; inner of 5 erecto-divergent lobes, $2 \mathrm{~mm}$ long, linear, alternating with outer corona. Pollinarium c. $0.3 \times 0.3 \mathrm{~mm}$. Follicles $6.8-12 \mathrm{~cm}$ long, tapering towards apex, glabrous. Seeds 6-8 $\mathrm{mm}$ long, brownish with yellow reflexed wings, comose; coma 1.5-2.5 cm long.

\section{Key to the varieties}

1. Leaves orbicular to elliptic-oblong, apiculate at apex var. bulbosa

1. Leaves linear-lanceolate, acute at apex var. lushii

Ceropegia bulbosa Roxb. var. bulbosa

Fig. 14

Flowering \& fruiting: June-December.

Chromosome number: $2 n=22$ (Raghavan \& Ansari, 1975).

Habitat: Grows along hills slopes in rock crevices and in association with Anogeissus latifolia, Woodfordia fruticosa Kurz and Prosopis juliflora DC.

Distribution: India, Bangladesh, Ethiopia, Kenya, North Yemen, Oman, Pakistan, Saudi Arabia, Somalia and Tanzania.

Specimens examined: INDIA, Andhra Pradesh, Cuddapah district, 1881, R.H. Beddome (CAL!); Nizamabad district, Nandipet R.F., 08.10.1988, B.R. Rao FAP 7198 (SKU!); Prakasam district, Badvel, 19.12.2007, B. Sadasivaiah \& M.V. Sureshbabu FAP 30519; C.S. Puram, 28.09.2008, B. Sadasivaiah \& S.K. Basha FAP 30834; Papinenipalli, 16.07.2008, B. Sadasivaiah \& S.K. Basha FAP 31029 (SKU!); Visakhapatanam district, way to Javapurami Kondal, 11.10.1972, G.V. Subbarao 42510 (CAL!). Gujarat, s.loc., 06.09.2013, M. Parmar E S.S. Kamable SSK 79 (SUK!). Karnataka, Chitradurga district, Chiradurga, 20.10.1979, T.M. Ramakrishna TMR 1347 (MH!); Kolar district, Mannagudda, 05.12.1977, T.M. Ramakrishna TMR 


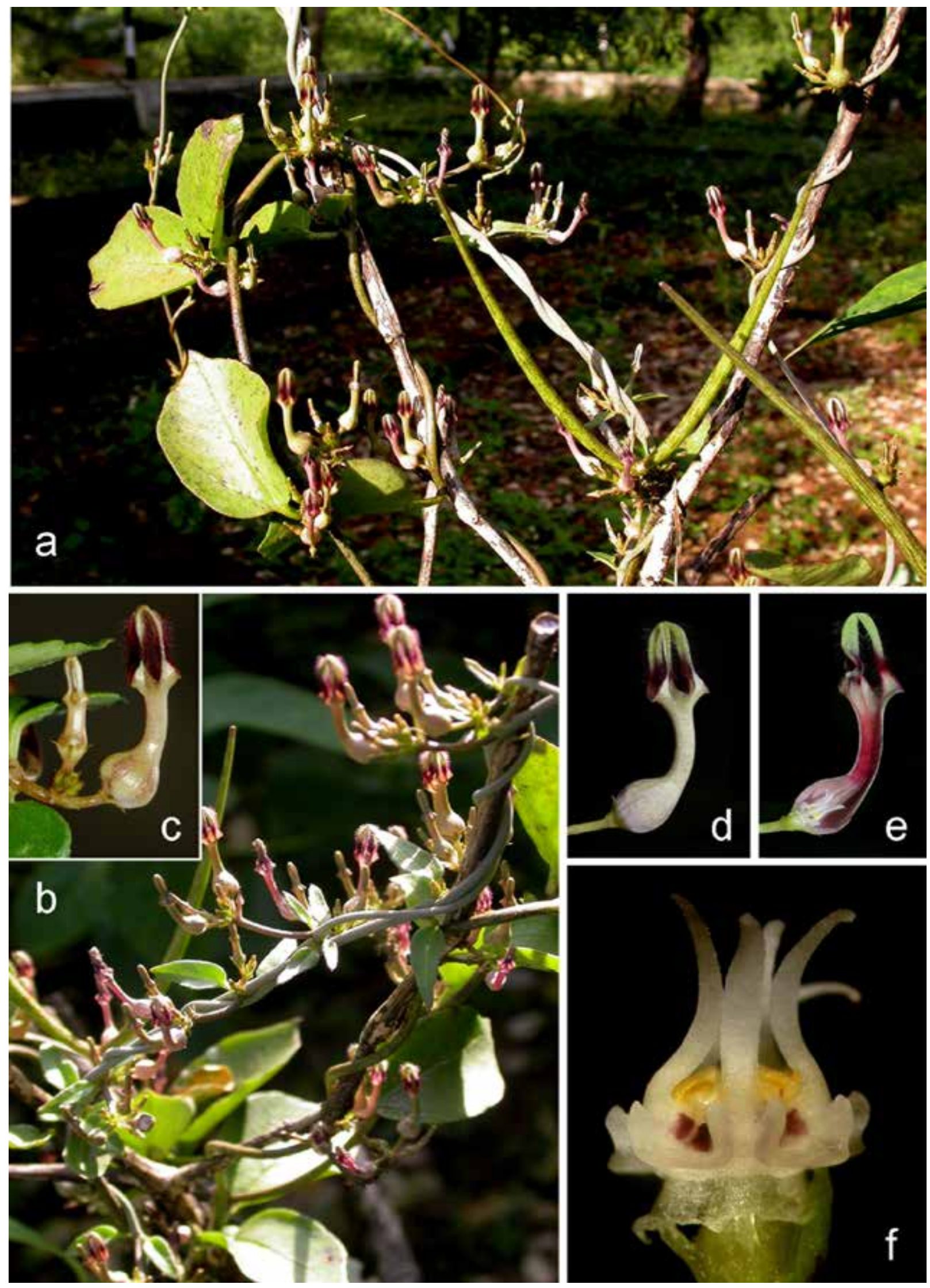

Fig. 14. Ceropegia bulbosa Roxb. var. bulbosa: a \& b. Habit; c. Inflorescence; d. flower; e. Flower L.S.; f. Corona-lateral view. 
1085; Kolar, 11.10.2014, S.S. Kambale \& S. Surveswaran SSK 326 (SUK!). Madhya Pradesh, Ambikapur district, Janakpur Forest, on the road to Jausennagar, 22.08.1974, G.S. Gupta 20561 (CAL!). Maharashtra, Akola district, 26.06.1977, S.Y. Kamble 150083 (BSI!); Aurangabad district, Ajintha forest, s.d., K.P. Janardhanan 100228; Mhaismal hills, 01.09.1964, K.P. Janardhanan 100092 (BSI!); Chandrapur district, near AmbitHira, 13.10.1972, B.M. Wadhawa 129271 (BSI!); Kolhapur district, s.d., M.M. Sardesai s.n. (SUK!); Ratnagiri district, Ganapatipule, 04.09.2003, M.Y. Kamble s.n. (SUK!); Nasik district, Chandwad, K.V.C. Gosavi SSK 19 (SUK!); Pune district, Junnar, Bhivade Khurd, 21.09.1968, K. Hemadri 117930A; Katraj Ghat, 13.08.1965, M.Y. Ansari \& B.V. Reddy 161201 (BSI!); Satara district, Kartikswami, 03.10.2004, M.Y. Kamble 2118; Vaduj, Amberi, P.R. Lawand E S.S. Kambale SSK 89; Kas, 10. 1994, M.P. Bachulkar-Cholekar 5590; Karad, 18.10.2011, S.S. Kambale 3134; Yenake, 13.07.2014, S.S. Kambale SSK 241(SUK!); Satara, 07.07.2013, S.S. Kamable SSK 51. Solapur district, Barshi, Gholavewadi, A.R. Gholave E S.S. Kambale SSK 80; Ibid., 01.09.2014, A.R. Gholave SSK 297 (SUK!); Thane district, Dhowla maki, Badlapur range, 24.09.1968, K.V. Billore 15355; Ibid., 11.1940, McCann 4945 (CAL!). Rajasthan, Baran district, Ramgarh hills, 25.09.1965, S. Sharma 13431 (BSI); Udaipur district, Guwalia hills, 28.09.1962, K.C. Kanodia 82209 (BSI!). Tamil Nadu, South Arcot district, 06.09.1899, C.A. Barber 832 (MH!).

Ceropegia bulbosa Roxb. var. lushii (Graham) Hook.f., Fl. Brit. India 4(10): 68. 1883; T. Cooke, Fl. Bombay 2: 176. 1908; Duthie, Fl. Gangetic Plain 2: 66. 1911; H. Huber, Mem. Soc. Broter. 12: 60. 1957; Gamble, Fl. Madras 4: 857.1957; Ansari, Fasc. Fl. India 16: 11. 1984; B.D. Sharma et al., Fl. Karnataka, Analysis 165. 1984; S.D. Deshp. et al., Fl. Mahabaleshwar, Maharashtra 1: 360. 1993; Hanumanthappa in Pull. \& Moulali, Fl. Andhra Pradesh 2: 584. 1997; Naik, Fl. Marathwada 1: 544. 1998; A.P. Jagtap \& N.P. Singh, Fasc. Fl. India 24: 217. 1999; M.R. Almeida, Fl. Maharashtra 3A: 229. 2001; F. Albers \& Meve, Ill. Handb. Succ. Pl. Asclepiadaceae 70. 2002; T.S. Nayar et al., Fl. Pl. Kerala 84. 2006; Karthik. et al., Fl. Pl. India 2: 160. 2009; Kambale \& S.R. Yadav, Asklepios 115: 29. 2013. Ceropegia lushii Graham, Bot. Mag. 61: t. 3300. 1834; Dalzell \& A. Gibson, Bombay Fl. 154.
1861. C. bulbosa var. lushii (Graham) Hook.f. 1883. Lectotype: Bot. Mag. 61: t. 3300.

C. acuminata Roxb., Pl. Coromandel 1: 12, t. 8. 1795; Dalzell \& A. Gibson, Bombay Fl. 153. 1861. Lectotype: Roxb. Pl. Coromandel 1: 12, t. 8.

C. esculenta Edgew., J. Proc. Linn. Soc., Bot. 6: 204. 1862. C. bulbosa Roxb. var. esculenta (Edgew.) Hook.f., Fl. Brit. India 4(10): 68. 1883. Type: not located or apparently none preserved.

C. bulbosa auct. Kamble \& S.G. Pradhan, Fl. Akola District, Maharashtra 130. 1988; B.V. Shetty \& V. Singh, Fl. Rajasthan 2: 476. 1991, non Roxburgh, 1795 .

Fig. 15

Vernacular name: Khapparkadu (Marathi).

Lamina linear to elliptic-lanceolate, acute at apex, rounded at base. Cymes 5-6-flowered, extraaxillary.

Flowering \& fruiting: July-October.

Chromosome number: $2 n=22$ (Raghavan \& Ansari, 1975).

Habitat: Grows along hills slopes, on rocky substratum.

Distribution: India (Andhra Pradesh, Gujarat, Karnataka, Maharashtra, Tamil Nadu), Bangladesh, Ethiopia, Kenya, North Yemen, Oman, Pakistan, Saudi Arabia, Somalia and Tanzania. Specimens examined: INDIA, Andhra Pradesh, Prakasam district, 22.09.1999, G. Sivaram FAP 20806 (SKU!). Gujarat, 06.09.2013, M. Parmar \& S.S. Kamable SSK 79 (SUK!). Karnataka, Chitradurga district, Chiradurga, 20.10.1979, T.M. Ramakrishna TMR 1347 (MH!); Kolar district, Mannagudda, 05.12.1977, T.M. Ramakrishna TMR 1083 (MH!). Maharashtra, Kolhapur district, Kagal, 02.08.2000, P.D. Mahekar 208; Ibid., s.d., V.N. Patil P 3278 (SUK!); Pune district, Pashan village, 06.08.1965, M.Y. Ansari \& B.V. Reddy 101216 (BSI!); Satara district, Kartikswami, 03.10.2004, M.Y. Kamble 2119 (SUK!); Kas, 10.1994, M.P.Bachulkar-Cholekar 5992; Solapur district, Gholavewadi, 01.09.2014, A.R. Gholave SSK 298 (SUK!). Tamil Nadu, Chennai, Chepauk, 27.11.1901, A.G. Bourne 3022 (CAL!).

Notes: Variants of C. bulbosa Roxb. have been described under different names by various authors viz. C. acuminata Roxb. (Roxburgh, 1795), C. lushii 


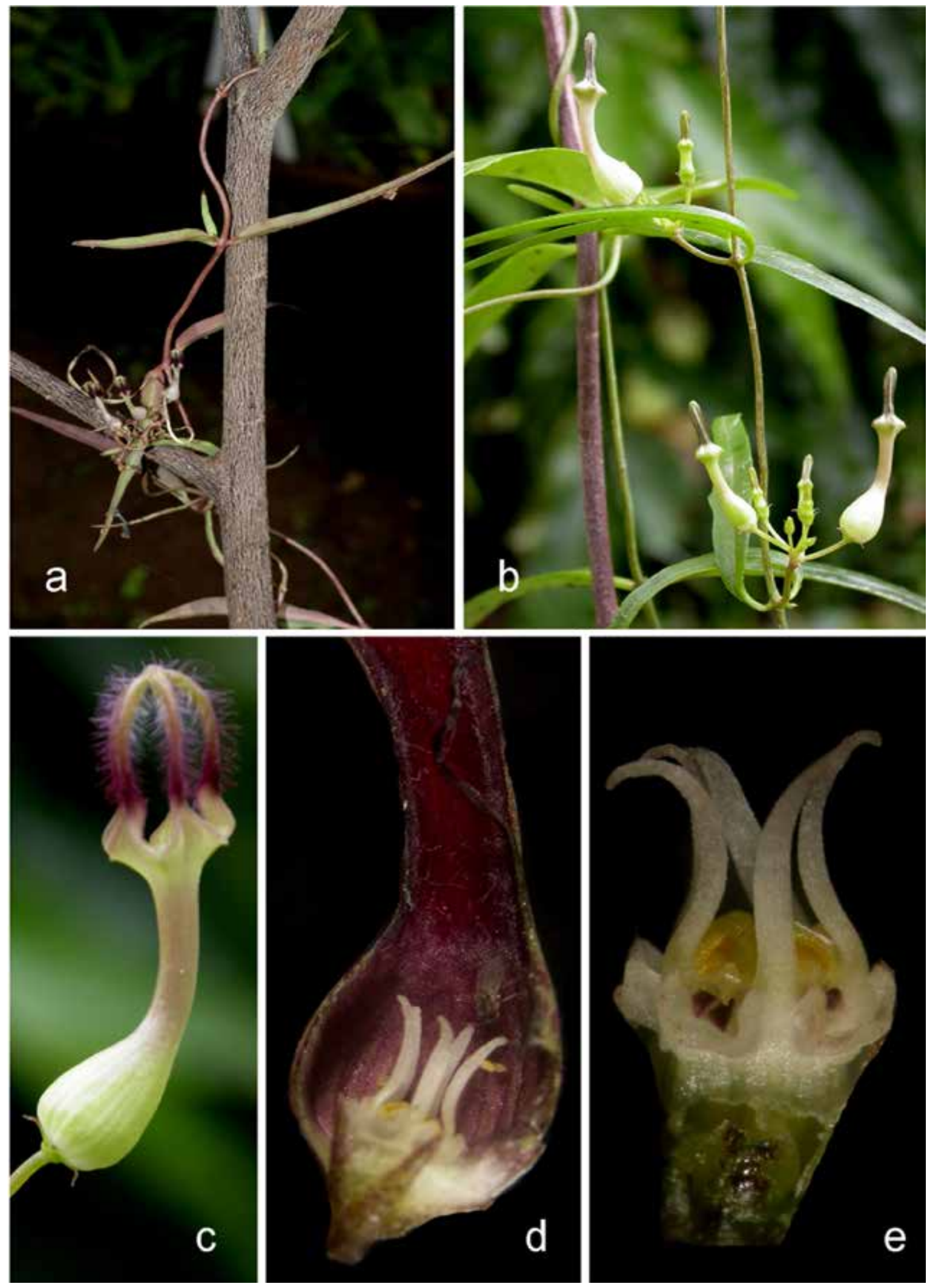

Fig. 15. Ceropegia bulbosa var. Iushii (Graham) Hook.f.: a \& b. Habit; c. Flower; d. Flower L.S.; e. Corona-lateral view. 
J. Graham (Graham, 1834) and C. esculenta Edgew. (Edgeworth, 1862). Hooker (1883) reduced the latter two as varieties of C. bulbosa. Subsequently, Huber (1957) synonymised all names under $C$. bulbosa Roxb. Ansari (1984) considered C. lushii and C. acuminata as conspecific and treated C. lushii as a variety (var. lushii) under C. bulbosa. He opined that this taxon can be distinguished by linear-lanceolate leaves with acute apex (as opposed to orbicularelliptic or oblong leaves with apiculate apex in var. bulbosa). Meve (2002) opinioned that the leaf character has little value and the form with linearlanceolate leaves may be considered as an ecotype. However, after thorough observations in the field and studies of herbarium specimens and taking consideration of the habitat preferences, C. lushii Graham is treated here as variety of C. bulbosa Roxb.

\section{Ceropegia sect. Indopegia}

Ceropegia sect. Indopegia H. Huber, Mem. Soc. Brot. 12: 28, 1957; Bruyns, S. African J. Bot. 112: 429. 2017. Type: Ceropegia hirsuta Wight \& Arn.

sect. Bupestris H. Huber, Mem. Soc. Brot. 12: 29. 1957. Type: Ceropegia ciliata Wight.

Erect to climbing tuberous herbs. Lamina cordate to ovate-lanceolate or lanceolate, petiolate, glabrous or pubescent. Cymes 2-12-flowered. Corona uni-seriate to bi-seriate.

Distribution: Twenty two species in the world. This section is endemic to the Western Ghats of Peninsular India (Table 1).

Key to the species of sect. Indopegia H. Huber

1. Erect herbs . .2

1. Climbing herbs . .6

2. Inner corona lobes clavate . .3

2. Inner corona lobes linear . .4

3. Corolla tube dilated up to the middle C. maccannii

3. Corolla tube dilated at base only C. panchganiensis

4. Corolla tube narrows at middle C. lawii

4. Corolla tube broad at middle 5

5. Cymes terminal; corolla lobes linear-oblong.... .C. rollae
5. Cymes extra-axillary; corolla ovate-obovate to elliptic C. sahyadrica

6. Sepals longer than corolla C. fantastica

6. Sepals shorter than corolla .7

7. Corona uni-seriate or outer inconspicuous ... 8

7. Corona bi-seriate .9

8. Corolla lobes form a depressed cage, c. $2 \mathrm{~cm}$ across C. huberi

8. Corolla lobes form a sub-globose cage, c. 0.5 cm across C. santapaui

9. Corolla lobes hairy 10

9. Corolla lobes glabrous 14

10. Corolla lobes equal to tube, coiled

.C. beddomei

10. Corolla lobes shorter than tube, not coiled ..11

11. Corolla tube glabrous within C. oculata

11. Corolla tube hairy within 12

12. Ring of hairs present at throat of dilated part of corolla tube C. barnesii

12. Ring of hairs absent at throat of dilated part of corolla tube 13

13. Pedicels hairy C. hirsuta

13. Pedicels glabrous C. vincifolia

14. Outer corona lobes entire C. odorata

14. Outer corona lobes bifid .15

15. Peduncles hairy/ puberulous 16

15. Peduncles glabrous .19

16. Pedicels glabrous C. evansii

16. Pedicels hairy 17

17. Outer corona sub-quadrate C. media

17. Outer corona saucer-shaped 18

18. Inner corona lobes ligulate C. ensifolia

18. Inner corona lobes spathulate C. ciliata

19. Corolla lobes equal to tube C. pullaiahii

19. Corolla lobes shorter than tube 20

20. Ring of hairs present at throat of dilated part of corolla tube C. omissa 
20. Ring of hairs absent at throat of dilated part of corolla tube C. bhatii

Ceropegia barnesii E.A. Bruce \& Chatterjee, Kew Bull. 3(1): 62. 1948; H. Huber, Mem. Soc. Brot. 12: 63. 1957; Ansari, Fasc. Fl. India 16: 10. 1984; S.R. Sriniv. in Henry et al. Fl. Tamil Nadu Ind. Ser I, Analysis 2: 82. 1987; M.P. Nayar \& Sastry, Red Data Book Indian Pl. 1: 51. 1987; A.P. Jagtap \& N.P. Singh, Fasc. Fl. India 24: 216. 1999; Karthik. et al., Fl. Pl. India 1: 160. 2009. Type: INDIA, South India, s.d., E. Barnes s.n. (holo K000857804 image!).

Perennial twining herbs. Root stock with fascicled roots. Stem glabrous. Lamina $18-24 \times 2-6 \mathrm{~cm}$, ovate-lanceolate, acuminate at apex, rounded at base, sparsely hairy above, glabrous beneath, densely hairy along margins. Cymes 3-5-flowered, extra-axillary; peduncles $2.5-4 \mathrm{~cm}$ long, slender, puberulous; bracts minute, subulate, hairy; pedicels $1.8-2.8 \mathrm{~cm}$ long, terete, puberulous. Sepals 6-7 $\mathrm{mm}$ long, subulate, glabrous. Corolla c. $4 \mathrm{~cm}$ long, glabrous throughout; tube $c .3 \mathrm{~cm}$ long, slightly curved, dilated at base with a ring of hairs within, funnel-shaped at throat, c. $2 \mathrm{~cm}$ across, narrow at middle, spotted within towards lower part; lobes 5 , c. $1.4 \mathrm{~cm}$ long, broadly triangular-ovate, subacute at apex, broad at base, connate at tip forming conical cage of $1.5-2 \mathrm{~cm}$ across, hairy within and along margins at tip. Corona bi-seriate; outer of 5 lobed, entire, ciliate; inner of 5 erect lobes, subclavate. Follicles c. $20 \times 0.5 \mathrm{~cm}$, slender, glabrous. Seeds $c .1 \mathrm{~cm}$ long, with inflexed margins, oblong, light brown, comose; coma 4-5 cm long, silky white.

Flowering: May-June.

Habitat: Occurs in evergreen forests.

Distribution: Endemic to Tamil Nadu, India.

Specimens examined: INDIA, Karnataka, South Canara, s.d., s.coll. s.n. (MH!). Tamil Nadu, Nilgiri, Pykara Fall, s.d., s.coll. s.n. (MH!).

Conservation status: Nayar and Sastry (1987) assessed it as Endangered due to habitat destruction. This plant is represented by only two sheets at $\mathrm{MH}$, one from Karnataka and another from Tamil Nadu and has not been collected since more than 40 years.

Notes: Ceropegia barnesii can be readily distinguished from other Indian ceropegias by its characteristically larger leaves $(18-24 \times 2-6 \mathrm{~cm})$. It is similar to C. intermedia Wight in its coronal structure but differs in having larger flowers with broader corolla lobes.

Ceropegia beddomei Hook.f., Fl. Brit. India 4: 75. 1883. Gamble, Fl. Madras 4: 859. 1921; H. Huber, Mem. Soc. Brot. 12: 66. 1957; Ansari, Fasc. Fl. India 16: 10. 1984; M.P. Nayar \& Sastry, Red Data Book Indian Pl. 1: 53. 1987; A.P. Jagtap \& N.P. Singh, Fasc. Fl. India 24: 217. 1999; T.S. Nayar et al., Fl. Pl. Kerala 84. 2006; Karthik. et al., Fl. Pl. India 1: 160. 2009. Type: INDIA, Kerala, Peerumade, 750 m, s.d., R.H. Beddome s.n. (holo K000894268 image!).

Fig. 16

Perennial twining herbs. Stem hispid. Lamina 7.5-20.5 × 0.6-0.9 cm, linear-lanceolate, acute at apex, narrow towards base, sparsely hairy on both surfaces; petioles $c .0 .6 \mathrm{~cm}$ long, pubescent. Cymes 2-3-flowered; peduncles 3.0-7.5 cm long, puberulous; bracts 3-5 mm long, linear; pedicels $1.0-1.5 \mathrm{~cm}$ long, puberulous. Sepals $0.8-1.0 \mathrm{~cm}$ long, linear. Corolla 5.5-9.8 cm long; tube 3.0-4.3 cm long, abruptly dilated at base; sub-cylindrical at middle, funnel-shaped above, white within at base, purple with white markings within the tube, equal to lobes; lobes $3.5-5.5 \mathrm{~cm}$, elongatetriangular, distally coiled hairy within and along margins, deep purple. Corona bi-seriate; outer of 5-bifid lobes, hairy within and along margins; inner of 5 erect-divergent lobes, deep purple, inflated above. Follicles c. $9 \mathrm{~cm}$ long, glabrous, blunt at tip, immature follicles green blotched with purple. Seeds 5-7 mm long, comose; coma 3.6-3.8 $\mathrm{cm}$ long, silky white.

Flowering \& fruiting: October-November.

Distribution: Endemic to Kerala, India.

Habitat: Grows in grasslands, in association with Cymbopogon sp.

Specimens examined: INDIA, Kerala, Idukki district, Calvary Mount, 05.10.1983, A.G. Pandurangan 79260; Kakki Dam, 10.10.1983, C.N. Mohanan 80104; Kulamavu, 03.10.1983, C.N. Mohanan 79260; Pachakkanam, 13.11.1975, K. Vivekananthan 46665 (MH!); Wayanad district, Gurukula Botanical Sanctuary, 23.11.2015, S.S. Kambale \& L. Joseph SSK 360 (SUK!).

Conservation status: Nayar and Sastry (1987) assessed 

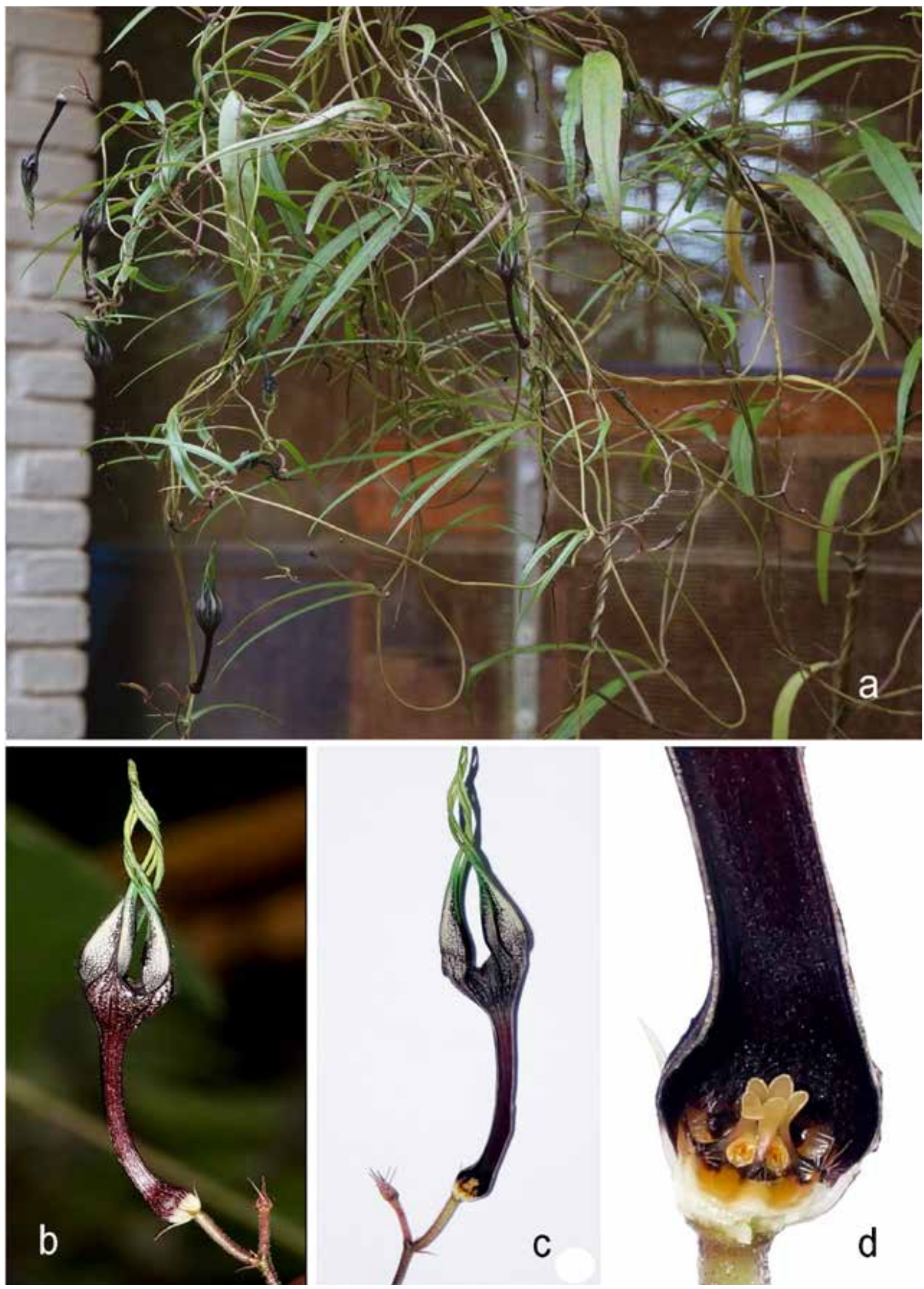

Fig. 16. Ceropegia beddomei Hook.f.: a. Habit; b. Flower; c. Flower L.S.; d. Corona. 
it as Endangered due to the habitat destruction.

Notes: Closely similar to C. barnesii but can easily be distinguished from the latter by its elongated hairy corolla lobes and linear-lanceolate leaves.

Ceropegia bhatii S.R.Yadav \& Shendage, Kew Bull. 65(1): 107. 2010; Kambale \& S.R. Yadav, Asklepios 115: 29. 2013. Type: INDIA, Karnataka, Davangere district, Malebennur Ghat, 15.09.2008, S.M. Shendage 2550 (holo CAL!; iso BSI!, SUK!).

Fig. 17

Perennial twining herbs. Rootstock tuberous, $2.5-3 \times 1.5-2 \mathrm{~cm}$; roots a few, fibrous. Stem $c .1 \mathrm{~m}$ long, 1-2 mm diam., terete, twining, unbranched, pubescent below, glabrous above. Lamina $4.2-7.3 \times 0.3-1.65 \mathrm{~cm}$, linear-lanceolate, hairy along margins, above and midrib beneath, dark green above, pale below; petioles 5-7 $\mathrm{mm}$ long, channeled above, ciliolate along margins, glabrous otherwise. Flowers solitary or 2-flowered cymes, extra-axillary; peduncles c. $5.5 \mathrm{~mm}$ long, glabrous; bracts c. $2.5 \times 1 \mathrm{~mm}$, linear-subulate, sparsely hairy at apex, glabrous otherwise; pedicels $c .1 .1$ $\mathrm{cm}$ long, terete, glabrous. Sepals c. $4.5 \times 0.35 \mathrm{~mm}$, subulate, glabrous. Corolla $c .4 .8 \mathrm{~cm}$ long; tube c. $2.8 \mathrm{~cm}$ long, slightly curved, gradually dilated towards base, funnel-shaped at throat, narrow at middle, lower $2 / 3^{\text {rd }}$ portion blotched with purple within, upper $1 / 3^{\text {rd }}$ portion striated with dark purple lines up to the throat within (at mouth of tube blotching is deep purple otherwise tube is yellowish-green within); lobes c. $2 \mathrm{~cm}$ long, linear, slightly reflexed on their back, connate at the tip forming an ovoid-cage, glabrous. Corona bi-seriate, stipitate, c. $4.5 \mathrm{~mm}$ long; outer of 5-bifid lobes, c. $2.3 \times 3 \mathrm{~mm}$, saucer-shaped, ciliate along margins, green-yellow; inner of 5 erectodivergent lobes, c. $2.8 \mathrm{~mm}$ long, sparsely hairy at the tip, deep purple. Pollinarium c. $0.2 \times 0.15 \mathrm{~mm}$. Follicles c. $8-9 \mathrm{~cm}$ long, tapering towards both ends, glabrous. Seeds c. $3.7 \times 0.15 \mathrm{~mm}$, ovateoblong; coma c. $1.7 \mathrm{~cm}$ long.

Flowering \& fruiting: August-November.

Habitat: Grows in grasslands of dry deciduous forests, in association with Anogeissus latifolia (Roxb. ex DC.) Wall. ex Guillem. \& Perr., Cymbopogon caesius (Hook. \& Arn.) Stapf, Dolichandrone spathacea Seem. and Tylophora fasciculata Buch.-Ham. ex Wight.
Distribution: Endemic to Karnataka, India.

Specimen examined: INDIA, Karnataka, Davangere district, Malebennur Ghat, 08.08.2011, S.S. Kambale 12 (SUK!).

Conservation status: So far the species is known from only type locality where the area of occupancy is less than $10 \mathrm{sq}$. $\mathrm{km}$. The fragmented populations observed contain a few undivided which are facing severe threat due to habitat destruction. Taking these facts into consideration and evaluation against criteria of IUCN (2017), species is assessed as Critically Endangered [CR: B1a,b (i, ii, v)].

Notes: This is the only species in series Attenuatae $\mathrm{H}$. Huber having a twining habit. Ceropegia bhatii is closely similar to C. noorjahaniae Ansari, but differs in its twining habit, 2-flowered cymes, gradually dilated corolla tube and straight corolla lobes.

Ceropegia ciliata Wight, Icon. Pl. Ind. Orient. 4(1): 15, t. 1262. 1848; Hook.f., Fl. Brit. India. 4: 73. 1883; Gamble, Fl. Madras 2: 858. 1921; H. Huber, Mem. Soc. Brot. 12: 68. 1957; Ansari, Fasc. Fl. India. 16: 13. 1984; S.R. Sriniv. in Henry et al., Fl. Tamil Nadu, Ser I, Analysis 2: 83. 1987; Manilal, Fl. Silent Valley 176. 1988; A.P. Jagtap \& N.P. Singh, Fasc. Fl. India 24: 219. 1999; F. Albers \& Meve, Ill. Handb. Succ. Pl. Asclepiadaceae 72. 2002; T.S. Nayar et al., Fl. Pl. Kerala 85. 2006; Karthik. et al., Fl. Pl. India 1: 161. 2009. Lectotype (designated by Kambale \& Yadav, 2014): INDIA. Tamil Nadu, Neilgherries [Nilgiris], Kaitie [Katie] Falls, s.d., R. Wight s.n. (K000894274 image!; isolectotype K000894273 image!).

Fig. 18

Perennial hirsute twining herbs. Rootstock tuberous; tubers $2-3 \times 1.5-2 \mathrm{~cm}$, roots fibrous. Stem c. $1 \mathrm{~m}$ long, unbranched, hirsute. Lamina 8.3-10.5 ×0.3-2.7 cm, ovate-lanceolate, attenuateacute at apex, rounded at base, coarsely pubescent on both sides, hairy on the veins beneath, ciliolate along margins; petioles c. $6 \mathrm{~mm}$ long, channeled above, hairy. Cymes umbellate, 6-15-flowered, extra-axillary; peduncles $3.3-10.5 \mathrm{~cm}$ long, hispid; pedicels $c .1 .8 \mathrm{~cm}$ long, hispid. Sepals $c .5 \mathrm{~mm}$ long, linear, hispid. Corolla $1.9-2.8 \mathrm{~cm}$ long, green; tube $1.2-1.8 \mathrm{~cm}$ long, straight-slightly curved, abruptly dilated at base, narrow at middle, cylindrical at throat, deep purple within at lower half, whitish green at upper-half; lobes $c$. 7-8 $\mathrm{mm}$ long, linear, connate at the tip forming globular cage, lobes 


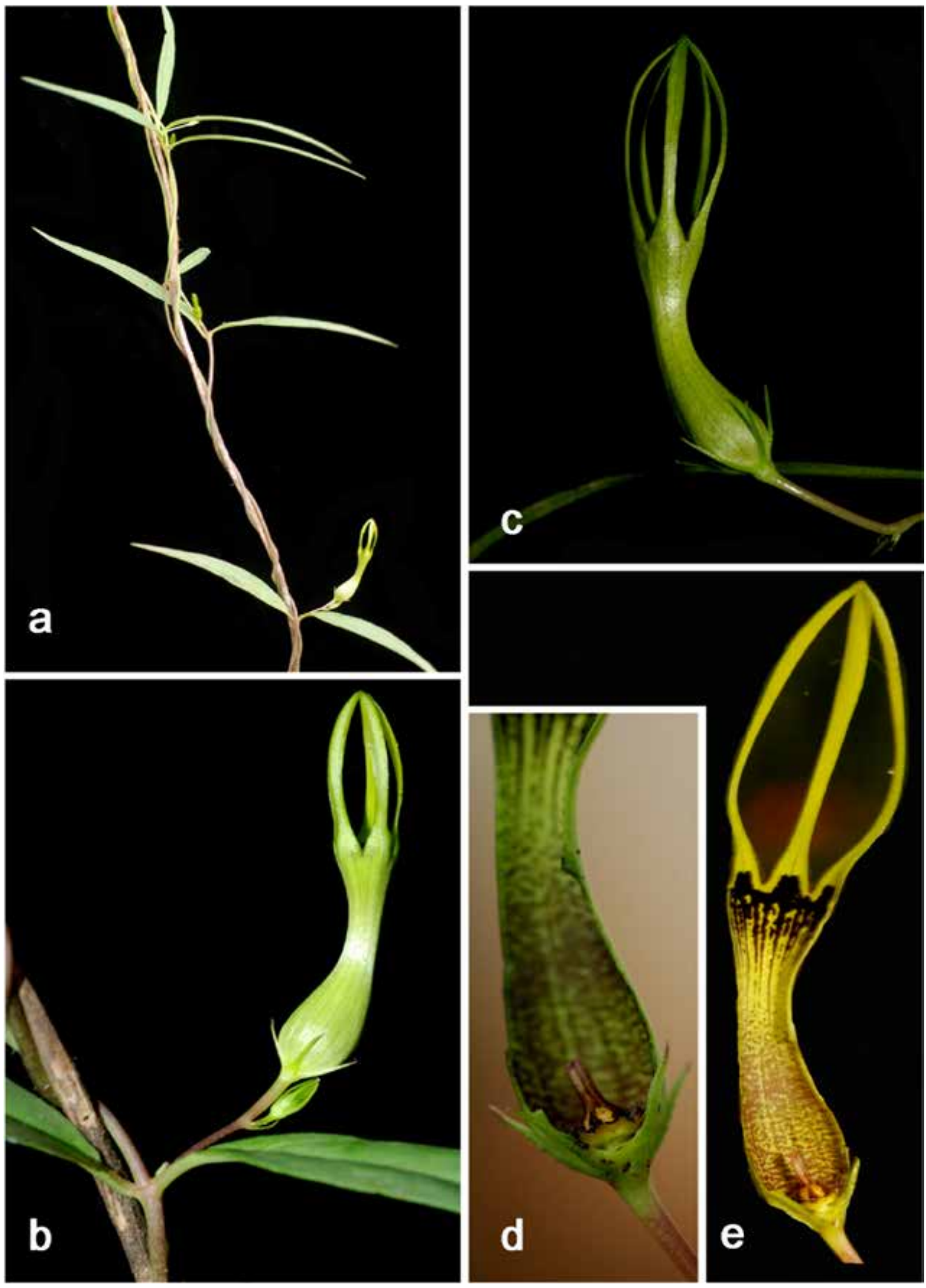

Fig. 17. Ceropegia bhatii S.R.Yadav \& Shendage: a. Habit; b. Inflorescence; c. Flower; d. Corona-lateral view; e. Flower L.S. 
slightly reflexed on their back, glabrous, faint green at upper $3 / 4^{\text {th }}$ portion and greenish white at lower $1 / 4^{\text {th }}$ part. Corona bi-seriate, stipitate; outer of 5-bifid lobes, c. $2 \times 1 \mathrm{~mm}$ across, saucer to bowlshaped, ciliate along margins and within; inner of 5 erect spathulate lobes, c. $1.6 \mathrm{~mm}$ long, alternate with outer corona. Follicles $c .8 \mathrm{~cm}$ long, tapering towards apex, blunt at tip.

Flowering: July-September.

Habitat: Grows amidst grasses on hill slopes.

Distribution: Endemic to southern Western Ghats
(Kerala and Tamil Nadu), India.

Specimens examined: INDIA, Kerala, Idukki district, Munnar, 18.08.2010, P.M. Sheeba 66250 (TBGT!). Tamil Nadu, Coimbatore district, Anamalay hills, 1921, s.coll. s.n. (MH!); Nilgiris district, s.loc., 09.1883, J.S. Gamble 12415; Ebbanad, 11.09.1970, G.V. Subbarao 6635 (MH!); Ibid., 09.08.2014, A.R. Gholave, S.R. Yadav \& S.S. Kambale SSK 260; Ibid., 19.09.2014, S.S. Kambale \& S.R. Yadav SSK 316; Ibid., 06.10.2014, S.S. Kambale \& S.R. Yadav SSK 322 (SUK!).

Conservation status: The populations are fragmented
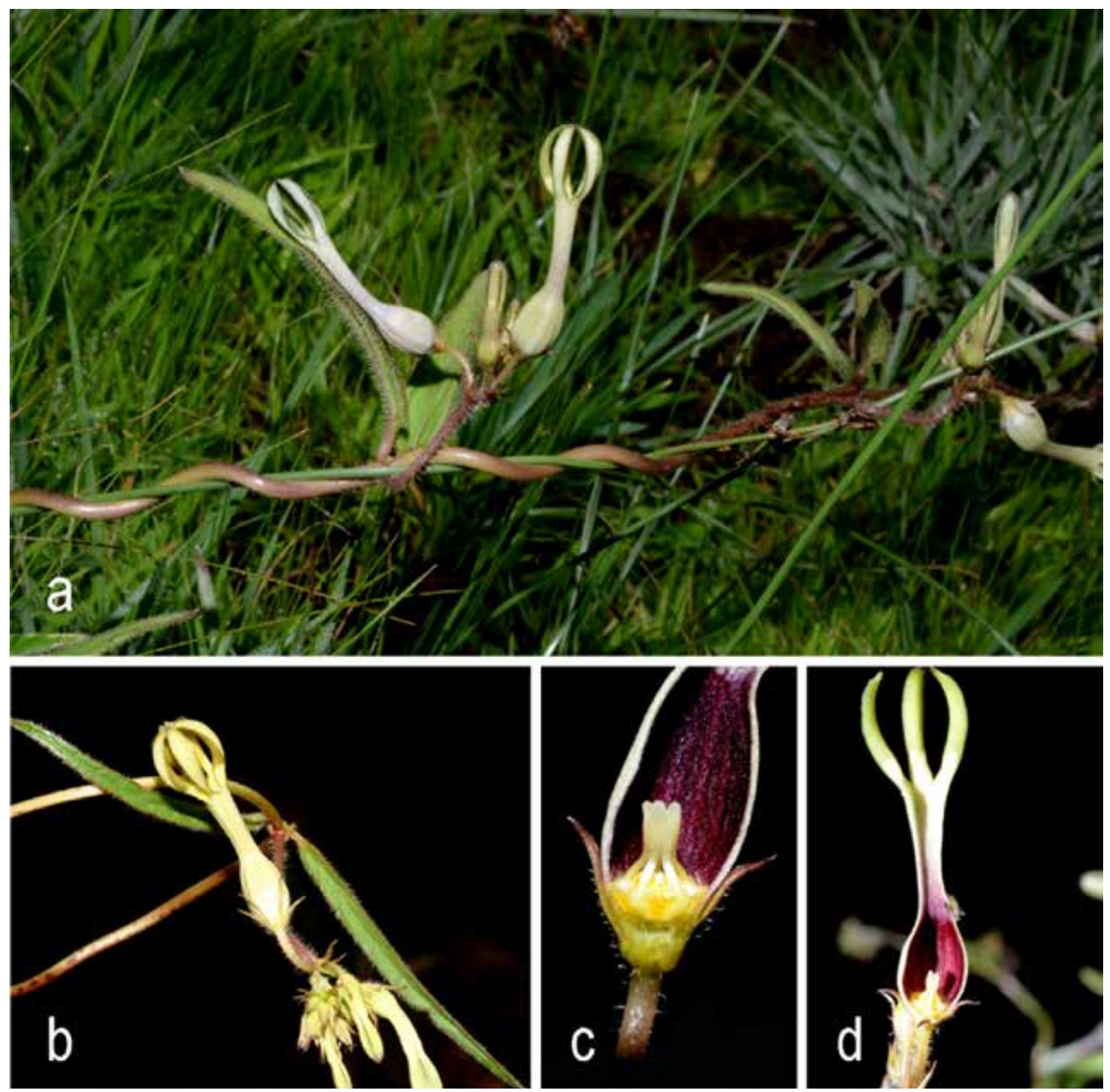

Fig. 18. Ceropegia ciliata Wight: a. Habit; b. Inflorescence; c. Corona; d. Flower L.S. 
and habitats are lost due to tea and coffee plantations. It is assessed here as Critically Endangered [CR: B2 a, b (iii,iv,v)].

Notes: Ceropegia ciliata is closely allied to C. ensifolia Bedd., but differs from it in having hirsute habit (vs. glabrous to puberulous), globular cage of corolla lobes ( $v s$. ovoid cage) and corolla lobes shorter than tube ( $v s$. corolla lobes and tube equal in length). It resembles to a great extent to C. hirsuta Wight \& Arn. in its vegetative morphology but differs in having globular cage of corolla lobes (vs. ovoid cage) and saucer-shaped outer corona with 5 shallowly bifid lobes (vs. bowl-shaped with deeply bifid lobes).

Ceropegia ensifolia Bedd., Madras J. Lit. Sci. Ser. 3(1): 52. 1864; Icon. P1. Ind. Or. 1: 15, t.173. 1874; Hook.f., Fl. Brit. India. 4: 75. 1883; Gamble, Fl. Madras. 4: 859. 1921; Ansari, Fasc. Fl. India. 16: 14. 1984; S.R. Sriniv. in Henry et al., Fl. Tamil Nadu Ind., Ser I, Analysis 2: 83. 1987; Bruyns, Rheedea 7(2): 111. 1997; A.P. Jagtap \& N.P. Singh, Fasc. Fl. India 24: 221. 1999; F. Albers \& Meve, Ill. Handb. Succ. Pl. Asclepiadaceae 77. 2002; T.S. Nayar et al. Fl. Pl. Kerala 85. 2006; Karthik. et al., Fl. Pl. India 1: 161. 2009. C. ciliata Wight subsp. ensifolia (Bedd.) H. Huber, Mem. Soc. Brot. 12: 69. 1957; Lectotype: INDIA, Tamil Nadu, Annamallay hills, R.H. Beddome s.n (K000894276 image!).

C. albiflora Hook.f., Fl. Brit. India 4(10): 75. 1883. Lectotype (designated by Singh, 2015): INDIA, Kerala, Thiruvananthapuram, Peerumade, 3500 ft., s.d., R.H. Beddome s.n. (K000894277 image!; isolectotype K000894278 image!, BM001014218 image!)

C. ciliata auct. Manilal, Fl. Silent Valley 176. 1988, non Wight, 1848.

Fig. 19

Perennial twining herbs. Rootstock a sub-globose tuber; roots fibrous. Stem branched, glabrous. Lamina $5.5-14 \times 0.4-0.7 \mathrm{~cm}$, ovate-lanceolate to elliptic-lanceolate, acute-acuminate at apex, rounded at base, coarsely hairy above and on the veins beneath, ciliolate along margins, glabrous otherwise; petioles $0.3-1.3 \mathrm{~cm}$ long, channeled above, decumbent, hairy. Cymes umbellate, 4-10-flowered, extra-axillary; peduncles 1.9-2.3 cm long, hispid; pedicels c. $6 \mathrm{~mm}$ long, hispid. Sepals c. $3 \mathrm{~mm}$ long, subulate, glabrous. Corolla 3-4.1 $\mathrm{cm}$ long, greenish-white; tube $0.8-1.9 \mathrm{~cm}$ long, straight, abruptly dilated at base, narrow at middle, sub-cylindrical at throat; lobes c. $2.2 \mathrm{~cm}$ long, linear, connate at the tip forming ovoid cage, lobes reflexed on back, glabrous, faint green. Corona biseriate; outer of 5 ciliate bifid lobes, saucer-shaped, emarginate; inner of 5 erect, ligulate lobes, alternate with outer corona. Follicles long, slender, tapering towards apex, blunt at the tip.

Flowering \& fruiting: August-September.

Habitat: Grows amidst grasslands on hill slopes.

Distribution: Endemic to southern Western Ghats of Kerala and Tamil Nadu, India.

Specimens examined: INDIA, Kerala, Idukki district, Kulamavu, 03.10.1983, C.N. Mohanan 79958 (MH!); Kottayam district, Kurisumala, 30.07.1985, V.T. Antony 1207 (MH!); Palakkad district, Karasuryamalai, 30.10.1976, E. Vajravelu 48769 (MH!); s.l., 20.10.2014, Prabhu Kumar SSK 329 (SUK!). Tamil Nadu, Kanyakumari district, Balamore, 05.10.1980, M.K. Vayal 68934 (MH!); Madurai district, way to high wavy Ghats, 06.09.1925, K.C. Jacob 17535 (MH!); Theni district, Meghamalai, 08.08.2014, S.S. Kambale E S. Surveswaran SSK 259; Ibid., 09.10.2014, S.S. Kambale \& S. Surveswaran SSK 323 (SUK!).

Notes: Ceropegia ensifolia is closely similar to $C$. ciliata, but differs from it in having glabrous habit, ovoid cage of corolla lobes, and longer corolla lobes.

Ceropegia evansii McCann, J. Bombay Nat. Hist. Soc. 45: 209. 1945; Ansari, Fasc. Fl. India 16: 15. 1984; A.P. Jagtap \& N.P. Singh, Fasc. Fl. India 24: 222. 1999; D.K. Mishra \& N.P. Singh, Endem. Threat. Pl. Maharashtra 132. 2001; A.P. Jagtap \& Das Das in N.P. Singh et al., Fl. Maharashtra, Dicot. 2: 350. 2001; M.R. Almeida, Fl. Maharashtra 3A: 230. 2001; F. Albers \& Meve, Ill. Handb. Succ. Pl. Asclepiadaceae 77. 2002; Karthik. et al., Fl. Pl. India 1: 161. 2009; Kambale \& S.R. Yadav, Asklepios 115: 31. 2013. C. evansii McCann var. evansii auct. H. Huber, Mem. Soc. Broter. 12: 67. 1957. Lectotype (designated by Kambale \& Yadav, 2015): INDIA, Maharashtra, Khandala, 13.09.1942, H. Santapau 920 B (BLAT image!).

Fig. 20

Vernacular name: Kurput (Marathi).

Perennial twining herbs. Rootstock tuberous; tubers 2-3.5 cm diam., sub-globose; roots fibrous. Stem c. 2-4 m long, usually single from 

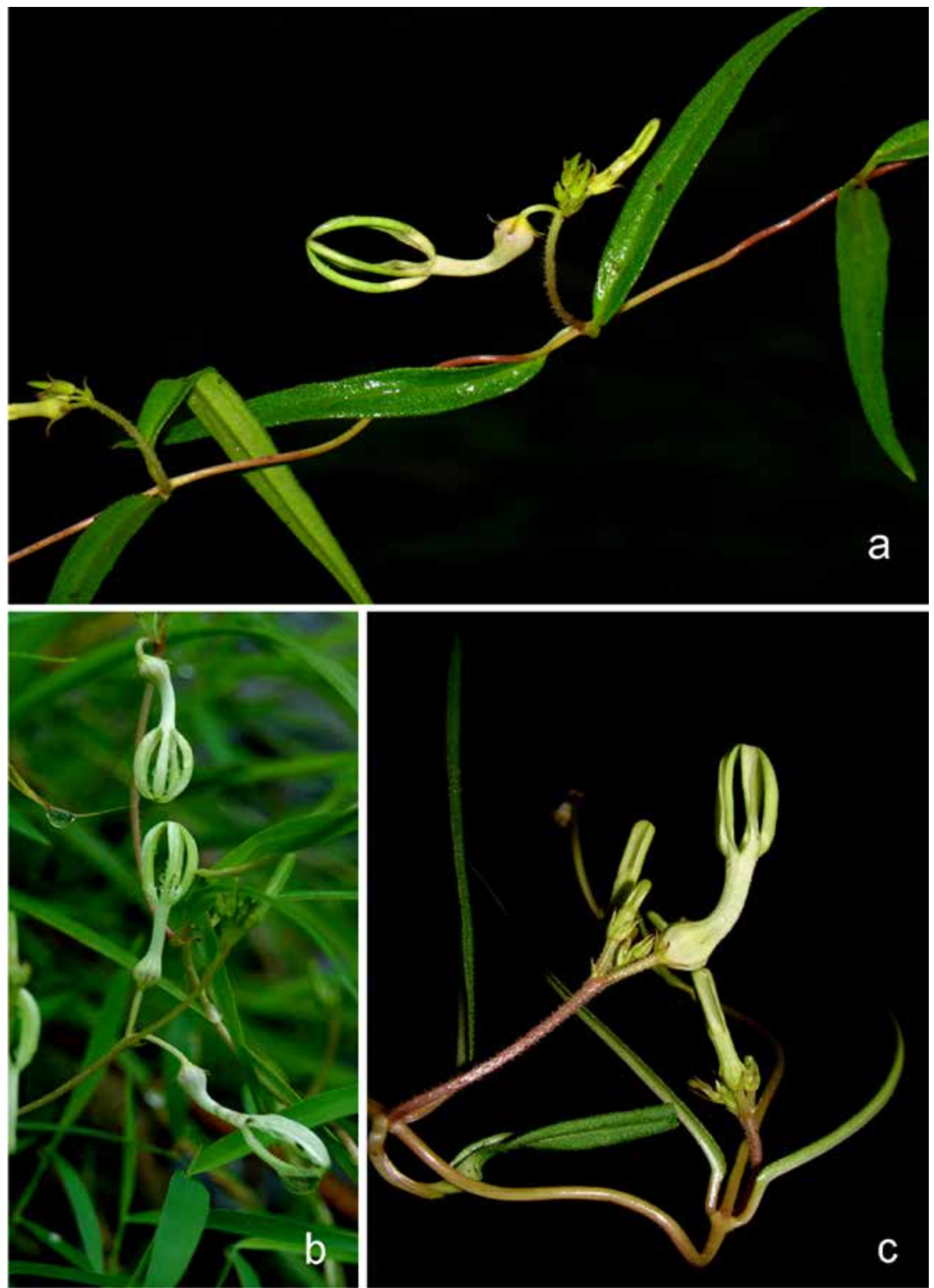

Fig. 19. Ceropegia ensifolia Bedd. : a \& b. Habit; c. Inflorescence (Photos K.M. Manudev). 
tuber, terete, glabrous-pubescent at young stage, green to dull purple. Lamina 6-14 × 1.3-7 cm, lower ovate-lanceolate, upper lanceolate, acute or acuminate at apex, rounded to sub-cordate at base with few glands on either side at the base, membranous, sub-coriaceous, hairy above and along margins and midnerve below, glabrous otherwise, dark green above, pale below; petioles $1.5-3 \mathrm{~cm}$ long, deeply channeled above, hairy along margins, glabrous otherwise. Cymes umbellate, 3-8-flowered, extra-axillary; peduncles $1.1-4 \mathrm{~cm}$ long, terete, puberulous; bracts $3-5 \mathrm{~mm}$ long, linear-subulate, glabrous, slightly pubescent along margins; pedicels $0.5-1.2 \mathrm{~cm}$ long, slender, glabrous. Sepals 5.5-6.5 mm long, linear-subulate glabrous. Corolla 3-4 cm long; tube $2.3-2.8 \mathrm{~cm}$ long, curved, abruptly dilated at base, cylindrical at middle, funnel-shaped at throat, glabrous within and outside, blotched with few green and brownish red spots at throat; lower half purple within, greyish outside; lobes $0.8-1 \mathrm{~cm}$ long, obovate-oblong, folded longitudinally on the back, connate at tip and forming an obovoid-cage, glabrous outside, puberulous within, lower half has downwardly pointed hairs, yellow at upper-half, white at lower half with green at margins. Corona bi-seriate; outer of 5-bifid lobes, $3 \times 4 \mathrm{~mm}$ across, saucer-shaped, ciliate within and along margins, yellow at base, deep purple along margins; inner of 5 linear lobes, c. $2.5 \mathrm{~mm}$ long, connivent, yellow with purple at the base. Pollinia minute, ellipsoidoblong, attached by very short caudicles to the corpusculum, yellow. Follicles in pairs usually unequal, blunt at tip, $7.5-15 \mathrm{~cm}$ long, tapering at both ends, glabrous. Seeds $c .7 \times 3 \mathrm{~mm}$, compressed, marginate, comose; coma c. $2 \mathrm{~cm}$ long, silky white.

Flowering \& fruiting: July-October.

Chromosome number: $2 n=22$ (Gosavi et al., 2012).

Habitat: Grows along hill slopes and amidst bushes, usually in association with Strobilanthes callosa Nees.

Distribution: Endemic to northern Western Ghats of Maharashtra, India.

Specimens examined: INDIA, Maharashtra, Kolhapur district, Patgaon, Faye, 04.08.2013, S.S. Kambale SSK 56; Ibid., 08.08.2014, S.S. Kambale SSK 258 (SUK!); Pune district, Khandala, below Dukes Nose, 08.1945, C. McCann 6527
(CAL!); Lonawala, Ambavane, Kate Pani forest, 27.07.1964, B.V. Reddi 99331 (BSI!); Ratnagiri district, Gothane, 28.07.2013, S.S. Kambale SSK 53; Ibid., 08.08.2014, S.S. Kambale \& A.R. Gholave SSK 257; Ibid., 19.09.2014, S.S. Kambale SSK 311 (SUK!).

Conservation status: As tubers are eaten by local inhabitants it is under threat. Very few populations have been located in the field. Nayar and Sastry (1987) assessed it as Vulnerable.

Ceropegia fantastica Sedgw., J. Indian Bot. 2: 124. 1921; Blatter \& McCann, J. Bombay Nat. Hist. Soc. 36: 536. 1933; H. Huber, Mem. Soc. Brot. 12: 64. 1957; B.D. Sharma et al., Fl. Karnataka, Analysis 166. 1984; Ansari, Fasc. Fl. India 16: 15. 1984; R.S. Rao, Fl. Goa, Diu, Daman, Dadra \& Nagarhaveli 2: 262. 1986; M.P. Nayar \& Sastry, Red Data Book Indian Pl. 1: 56. 1987; A.P. Jagtap \& N.P. Singh, Fasc. Fl. India 24: 222. 1999; A.P. Jagtap \& Das Das in N.P. Singh et al., Fl. Maharashtra, Dicot. 2: 350. 2001; F. Albers \& Meve, Ill. Handb. Succ. Pl. Asclepiadaceae 77. 2002; Karthik. et al., Fl. Pl. India 1: 161. 2009; Kambale \& S.R. Yadav, Asklepios 115: 33. 2013. Lectotype (designated by Singh, 2015): INDIA, Karnataka, Sulgeri, North Kanara, 08.1918, T.R.D. Bell 4252 (K000894267 image!; isolectotype K000894266).

Fig. 21

Perennial twining herbs. Rootstock tuberous; tubers sub-spherical, $2.5-3 \mathrm{~cm}$ in diam.; roots fibrous. Stem twining, c. $3 \mathrm{~m}$ high, usually 1 from single tuber, $2-3 \mathrm{~mm}$ in diam, cylindrical, glabrous. Lamina 8.3-14.0 × 6.5-10 cm, lower ones large, ovate-lanceolate, caudate-acuminate at apex, rounded-cordate at base, wavy along margins, membranous, pubescent above and along margins, glabrous beneath, with glands at the base of lamina, upper ones small 6.3-13.3 $\times$ $1.8-4.3 \mathrm{~cm}$ long, lanceolate, acute at apex, cuneate at base, distinctly curved at apex, hairy along margins, glabrous otherwise; petioles $0.7-3.9 \mathrm{~cm}$ long, channeled above, glabrous. Cymes subumbellate, extra-axillary; peduncles $1.8-8.3 \mathrm{~cm}$ long, terete, hirsute, deep purple. Flowers 6-10; bracts $1.5-1.8 \times 0.15 \mathrm{~cm}$, linear-subulate, hairy at the tip, glabrous otherwise, purple; bracteoles $0.5-1 \times 0.1 \mathrm{~cm}$, linear-subulate, glabrous, purple; pedicels $0.8-2.4 \mathrm{~cm}$ long, terete, glabrous, purple. Sepals 4.3-5.1 ×c. $0.1 \mathrm{~cm}$, linear-subulate, slightly hooked at tip, exceeding corolla, glabrous, purple. 

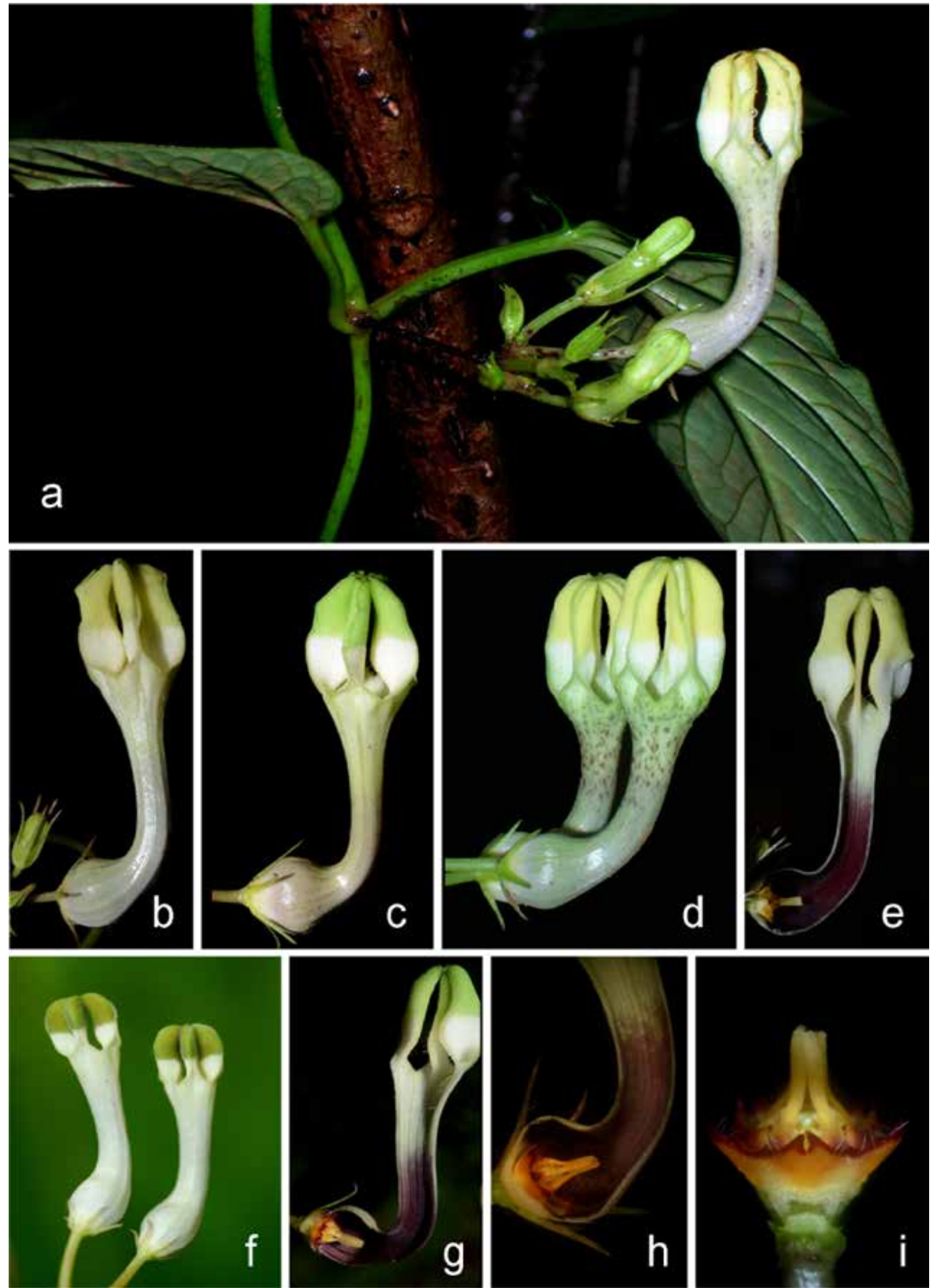

Fig. 20. Ceropegia evansii McCann: a. Habit; b-d \& f. floral variations; e, g \& h. L.S of flower; i. Corona-lateral view. 

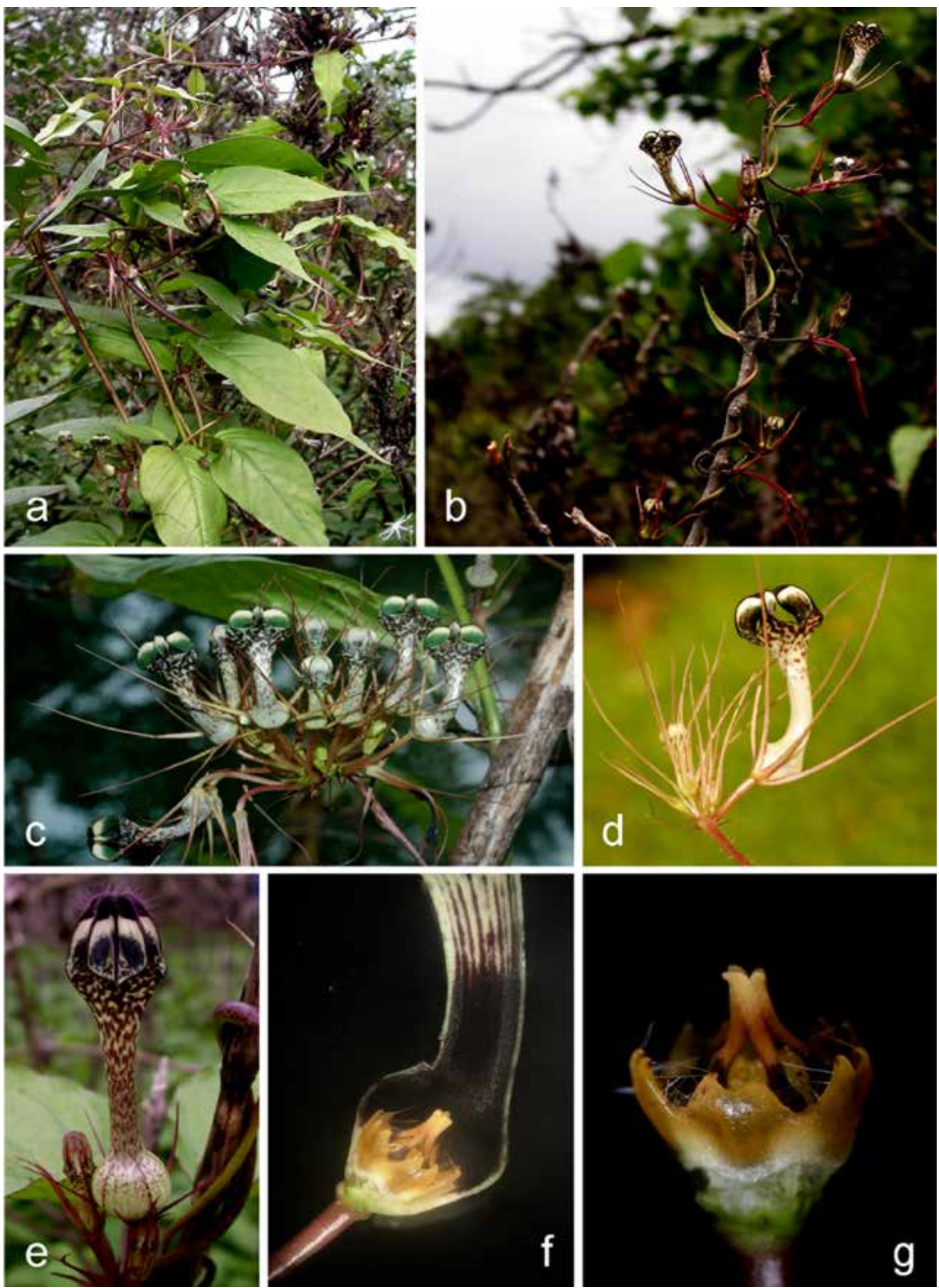

Fig. 21. Ceropegia fantastica Sedgw.: a \& b. Habit; c. Inflorescence; d. Flower; e. Variant flower with calyx shorter than corolla; f. Flower L.S.; g. Corona-lateral view. 
Corolla 3.2-4.4 cm long; tube 2.5-3.4 cm long, slightly curved, abruptly dilated towards base, subcylindrical to funnel-shaped at mouth, funnelshaped above, hairy at mouth with downwardly pointed trichomes, faint green at dilated part, blotched with deep purple from base to upward, blotching maximum at upper-half up to the base of corolla lobes, deep purple at lower $2 / 3^{\text {rd }}$ portion within, lower half of dilated part cream-yellow coloured, upper $1 / 3^{\text {rd }}$ portion tube blotched with purple; lobes $0.7-1.0 \mathrm{~cm}$ long, folded at their back, connate at the tip to form sub-globose head, hairy along margins and within, density of hairs is high at the top, greenish interspersed with white at the middle of lobe, purple along margins within, variously coloured, blotching with purple is high. Corona bi-seriate, stipitate; outer of 5-bifid lobes, c. $3 \times 3.5 \mathrm{~mm}$, bowl to cupular-shaped, ciliate along margins and within, yellow; inner c. 2.5 $\mathrm{mm}$ long, connivent at tip, yellow at upper-half with purple blotching, deep purple at lower half. Pollinarium c. $2.5 \times 3 \mathrm{~mm}$. Follicles 9-9.5 $\times$ c. 0.3 $\mathrm{cm}$, ash coloured; seeds $c .8 \times 3 \mathrm{~mm}$, brownish with yellow reflexed wings, comose; coma c. $2 \mathrm{~cm}$ long, silky white.

Flowering \& fruiting: August-September.

Habitat: Grow in open semi-evergreen forests amidst bushes and along forest edges in wet evergreen forests.

Distribution: Endemic to northern Western Ghats (Goa, Karnataka, Maharashtra), India.

Specimens examined: INDIA, Goa, South Goa district, Canacona, Ordofond forest, way to Tudal, 24.08.1963, K.C. Kanodia BSI 89489 (BSI!); Loliem, s.d., V.C. Joshi \& S. Rajkumar s.n. (Goa University Herbarium); Sanguem, Netravali, 10.07.2012, S.S. Kambale SUK 2575; Ibid., 21.08.2014, S.S. Kambale SSK 282 (SUK!). Karnataka, North Canara, way from Kadra to Sulgeri, 16.09.2011, A.N. Chandore ANC 1431 (SUK!). Maharashtra, Kolhapur district, Ajara, s.d., S.P. Gaikwad 468; way from Ajara to Amboli, 04.09.1999, S R. Yadav s.n.; Gavase, 16.07.2011, S.S. Kambale SUK 2583; Ibid., 15.08.2014, S.S. Kambale \& A.N. Chandore SSK 269; 19.09.2014, S.S. Kambale SSK 317 (SUK!); Ratnagiri district, Gothane, 19.09.2014, S.S. Kambale SSK 312 (SUK!); Sindhudurg district, Amboli, s.d,. S.R. Yadav 5913 (SUK!).
Conservation status: Nayar and Sastry (1987) assessed it as Endangered or possibly Extinct based on the data available then. However, the species is known from Goa, Karnataka and Maharashtra by few populations. The habitat modification is the major threat to the species.

Notes: Ceropegia fantastica closely resembles C. evansii and C. oculata in having hirsute, ovatelanceolate leaves. However, it can be distinguished by its considerably longer sepals.

Ceropegia hirsuta Wight \& Arn., Contr. Bot. India 30. 1834; Hook.f., Fl. Brit. India 4: 71. 1883; T. Cooke, Fl. Bombay 2: 242. 1905; Gamble, Fl. Madras 4: 859. 1921; H. Huber, Mem. Soc. Broter. 12: 63. 1957; Stevens in Saldanha \& Nicolson, Fl. Hassan Dist. 444. 1976; K.M. Matthew, Fl. Tamilnadu Carnatic 2: 936. 1983; B.D. Sharma et al., Fl. Karnataka, Analysis 166. 1984; Ansari, Fasc. Fl. India 16: 16. 1984; S.R. Sriniv. in Henry et al., Fl. Tamil Nadu Ind., Ser I, Analysis 2: 83. 1987; Kamble \& S.G. Pradhan, Fl. Akola District, Maharashtra 130. 1988; Manilal, Fl. Silent Valley 177. 1988; B.V. Shetty \& V. Singh, Fl. Rajasthan 2: 478. 1991; S.D. Deshp. et al., Fl. Mahabaleshwar, Maharashtra 1: 360. 1993; Hanumanthappa in Pull. \& Moulali, Fl. Andhra Pradesh 2: 585. 1997; H.O. Saxena \& Brahmam, Fl. Orissa 2:1087. 1995; Bruyns, Rheedea 7(2): 113. 1997; Naik, Fl. Marathwada 1: 545. 1998; A.P. Jagtap \& N.P. Singh, Fasc. Fl. India 24: 223. 1999; F. Albers \& Meve, Ill. Handb. Succ. Pl. Asclepiadaceae 81. 2002; Boonjaras \& Thaithong, Thai For. Bull. (Bot.) 31: 1. 2003; A.P. Jagtap \& Das Das in N.P. Singh et al., Fl. Maharashtra, Dicot. 2: 350. 2001; T.S. Nayar et al., Fl. Pl. Kerala 85. 2006; Karthik. et al., Fl. Pl. India 1: 161. 2009; Kambale \& S.R. Yadav, Asklepios 115: 33. 2013. Lectotype (designated by Ansari, 1984): INDIA, Tamil Nadu, Nilgiris, s.d., R. Wight Cat. n. 1510 (K000894263 image!; isolectotype E00179553 image!).

C. hirsuta var. stenophylla Hook.f., Fl. Brit. India 4: 72. 1883; Gamble, Fl. Madras 4: 859. 1921; K.M. Matthew, Fl. Palni Hills, S. India 2: 803. 1999. Type: Not located or apparently not known.

C. jacquemontiana Decne., Prodr. 8: 641. 1844; Dalzell \& A. Gibson, Bombay Fl. 153. 1861. C. hirsuta var. jacquemontiana (Decne.) Hook.f., 4: 72. 1883. Type: Not located or apparently not known. 
C. ophiocephala Dalzell, Hooker's J. Bot. Kew Gard. Misc. 2: 259. 1850; Dalzell \& A. Gibson, Bombay Fl. 154. 1861. Type: Not located or apparently not known.

C. hispida Blatt. \& McCann, J. Bombay Nat. Hist. Soc. 35: 409. 1931. Type: INDIA, Maharashtra, Western Ghats, Panchgani, near Goudoli, 06.10.1930, C. McCann \& Fernandez 3566 (holo BLAT image!).

Fig. 22

Vernacular names: Khaloola (Hindi), Haamana (Marathi), Khilora (Rajasthani).

Perennial twining herbs. Rootstock tuberous; tubers $2-4.3 \mathrm{~cm}$ in diam., globose-depressed, roots fibrous. Stem 30-200 cm long, usually 1 from single tuber, slender, unbranched, hirsute. Lamina variable, lower ones $2-11.2 \times 1.8-7 \mathrm{~cm}$, ovatelanceolate, acute at apex, rounded at base, upper ones ovate-lanceolate, $4.8-8.2 \times 0.8-4.3 \mathrm{~cm}$, acute at apex, rounded-cordate at base, hirsute on either sides, densely hairy along margins; petioles $0.5-2.8$ $\mathrm{cm}$ long, channeled above, hairy. Cymes subumbellate, 3-5-flowered, lateral; peduncles 0.6$2.8 \mathrm{~cm}$ long, terete, hirsute; bracts $0.6-1 \mathrm{~cm}$, linear, hairy; bracteoles $0.1-0.15 \mathrm{~cm}$, usually glabrous; pedicels c. $0.6-1.2 \mathrm{~cm}$, pubescent. Sepals $0.5-2 \mathrm{~cm}$ long, linear-subulate, hirsute. Corolla $3.2-7.2 \mathrm{~cm}$ long; tube 2-4 cm long, dilated at base, funnelshaped at throat, glabrous, downwardly pointed hairs at the mouth and at the lower $2 / 3^{\text {rd }}$ portion, yellowish-green, externally blotched with dark purple spots from middle to upward deep purple at lower $3 / 4^{\text {th }}$ portion and with purple blotches at upper $1 / 4^{\text {th }}$ portion; lobes $0.8-2.3 \mathrm{~cm}$ long, broadly oblong or oblong obovate, hairy along margins and from inner side yellowish-green, sometimes purple in colour or pale yellow. Corona, bi-seriate, stipitate; outer of 5-bifid lobes, bowl-shaped, longer than gynostegium, c. $4 \times 5 \mathrm{~mm}$, ciliate along margins and within, dark brown-black coloured along margins, dark yellow in the middle; inner of 5 connivent-slightly divergent lobes, $4-5 \mathrm{~mm}$ long, yellow, purple at the base. Follicles $8-12 \times c$. $0.35 \mathrm{~cm}$, glabrous, tapering towards apex.

Flowering \& fruiting: June-December.

Habitat: Grows amidst bushes along hill slopes of dry regions, on lateritic plateaus and grassy hill slopes.
Distribution: Peninsular India and Thailand.

Specimens examined: INDIA, Andhra Pradesh, Kadapa district, Talakona, 25.11.2007, B.R.R.S. Rao, B. Sadasivaiah \& S.K. Basha F.A.P. 30388 (SKU!, BSID!); Ranga Reddy district, Ananthagiri Reserve forest, 20.08.1994, M.S. Mohamad F.A.P. 11059 (SKU!); Srikakulam district, Rampa hills, 09.1920, V. Narayanaswami 869 (CAL!). Goa, South Goa district, Paroda hills, 09.09.2007, A. Prabhugaonkar \& M.K. Janarthanam 536 (Goa University Herbarium!). Jharkhand, Giridh district, Parasnath Wildlife Sanctuary, 02.10.2004, V. Ranjan \& K.L. Maity 37219; Hazaribagh district, Hazaribagh National Park, 07.09.1993, S.K. Das \& Party 22447; Near Raja Rappa forest, 20.09.1993, S.K. Das \& Party 22541 (CAL!). Karnataka, Belgaum district, Appachiwadi, 17.08.2012, A.R. Gholave \& S.S. Kambale SSK 10 (SUK!); Chamrajnagar district, Mysore to Gundulupet, 29.08.1964, B.D. Naikthani 21297 (MH!); Chikmaglur district, way from Chikmaglur to Shimoga, 10.09.2011, S.S. Kambale SUK 2598 (SUK!); Hassan district, Segegudda, 02.10.1977, T.M. Ramakrishna TMR 1012 (MH!, CAL!); Kodagu district, Pushpagiri hills, 20.10.2013, P.R. Kshirsagar \& S.S. Kambale SSK 93 (SUK!); Tumkur district, Arai-gudenahalli, 08.11.2013, P.R. Lawand, A.R. Gholave \& S.S. Kambale SSK 202 (SUK!). Kerala, Idukki district, Kulamavu to Meenmutty, s.d., C.N. Mohanan 76008 (MH!); Palakkad district, Silent Valley National Park, 20.11.2011, A.R. Kulavmode \& S.S. Kambale SUK 2568 (SUK!); Pathanamthitta district, Nilakkal, 08.06.1982, K.S.P. Kumar SV 10902 (CALI!). Madhya Pradesh, Hoshangabad district, Panchmarhi road, Bori Reserve forest, 06.10.1960, J. Joseph 11284 (MH!). Maharashtra, Akola district, Palodi, 02.09.1977, S.Y. Kamble 150373 (BSI!); Amravati district, Belkund, 28.08.1976, M.Y. Ansari 144149 (BSI!); Kolhapur district, Gaganbawda, 25.08.1999, S.R. Yadav s.n.; Ajara, 27.09.1999, S.R. Yadav s.n.; Ibid., 05.08.2003, S.R. Yadav s.n.; Katyayani, 19.07.2011, S.S. Kambale SUK 2584 (SUK!); Malabar \& Konkan region, s.loc., s.d., Stocks \& Law s.n. (CAL!); Panhala, 09.08.2014, S.S. Kambale \& B.U. Jadhav SSK 261(SUK!); Mumbai Sub-urban district, Trombay, 02.07.1970, B.N. Maheshwari 72944 (BSI!); Pune district, Chakan, 03.09.1962, K.P. Janardhanan 780081; hills around Pune, 18.08.1902, R.K. Bhide 793; Lonavla, 29.09.1914, N.P. Par s.n.; Near Aundh, 23.09.1921, 

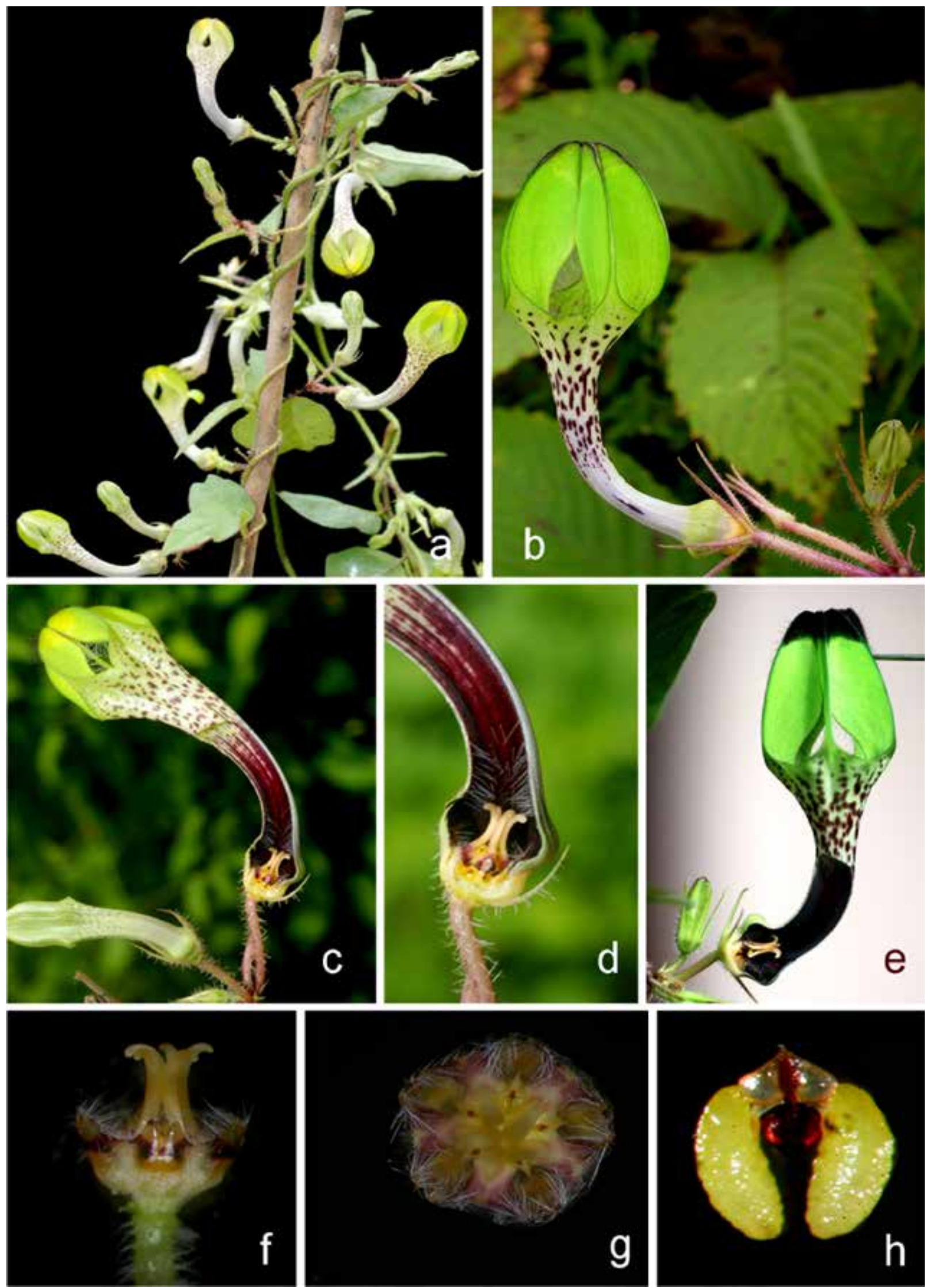

Fig. 22. Ceropegia hirsuta Wight \& Arn.: a. Habit; b. Flower; c-e. Flower L.S.; f. Corona-lateral view; g. Corona-top view; h. Pollinarium. 
R.S. Shinde s.n.; Pashan, 26.08.1988, Lukuram s.n. (BSI!); Ratnagiri district, Ratnagiri, 05.09.1999, V.T. Patil 404 (SUK!); Sangli district, Miraj, Pandoba hill, A.N. Londhe 170398; Tasgaon, Sagareshwar forest, 28.07.1990, A.N. Londhe 170601 (BSI!); Satara district, Karad, Shamgaon Ghat, 18.09.2013, P.R. Lawand \& S.S. Kambale SSK 90; Kartikswami hills, Amberi, 22.08.1990, S.R. Yadav s.n.; Ibid., Amberi, 22.08.2010, S.S. Kambale SUK 2567; Khindwadi, 09.1992, H.S. Patil 3515 (SUK!); Pandavgarh, 11.08.1984, S.D. Deshpande 169037 (BSI!); Satara, 07.07.2013, S.S. Kambale SSK 52; Yenake, 13.07.2014, S.S. Kambale \& S.B. Chaugule SSK 242 (SUK!); Sindhudurg district, Vengurla, 28.08.2011, S.S. Kambale SUK 2596 (SUK!). Odisha, Ganjam district, s.loc., s.d., C.E.C. Fischer 4 (CAL!). Rajasthan, Mount Abu, 16.07.1957, s.coll. 42857 (BSI!). Tamil Nadu, Coimbatore district, Anamalay hills, 1921, J.S. Gamble s.n.; Kuridimalai, 13.12.1956, K. Subramanyan 1768 (MH!); Dharmpuri district, Devarbetta, 29.10.1981, K.M. Matthew, S.J. Britto \& N. Rani 28493 (RHT!); Dindigul district, Palani hills, 11.1856, R.H. Beddome 107 (CAL!); Ibid., 22.06.1898, Bourne 1603; Madurai district, Anna, 25.08.1985, S.J. Britto 42139 (RHT!); Nilgiris district, Moyar Reserve forest, 18.08.1970, B.D. Sharma 35593; Sirur to Ebbanad 14.11.1970, G.V. Subbarao 37227 (MH!). West Bengal, Purulia district, Chota Nagpur, 09.1896, D. Prain s.n.; Matha hills, 11.09.1964, K.C. Maliek s.n.; Gopalpur forest, 25.08.1966, K.C. Maliek 438 (CAL!).

Conservation status: It is distributed in Peninsular India and Thailand. However due to the anthropogenic pressure such as habitat modification, food value of tubers, it is under threat. Due to inadequate information, it is treated under Data Deficient (DD).

Notes: Ceropegia hirsuta is exceptionally variable in its morphology. Populations from different parts of the Western Ghats shows much variation in plant size, leaf shape, length of flowers, colour of corolla and shape of corolla lobe cage. Shape of the lamina varies from ovate-elliptic, lanceolate to linear; colour of the flowers varies from green to yellow (in drier parts) and the corolla lobe from conical to ovoid cages. A comparative account of variations in the floral structure is depicted in Figure 23.

Ceropegia huberi Ansari, Bull. Bot. Surv. India 10. 219. 1969; Ansari, Fasc. Fl. India 16: 17. 1984; M.P.
Nayar \& Sastry, Red Data Book Indian Pl. 1: 58. 1987; A.P. Jagtap \& N.P. Singh, Fasc. Fl. India 24: 225. 1999; D.K. Mishra \& N.P. Singh, Endem. Threat. Pl. Maharashtra 133. 2001; A.P. Jagtap \& Das Das in N.P. Singh et al., Fl. Maharashtra, Dicot. 2: 351. 2001; Almeida, Fl. Maharashtra. 3A: 232. 2001; Karthik. et al., Fl. Pl. India 1: 162. 2009; Kambale \& S.R. Yadav, Asklepios 115: 31. 2013. Type: INDIA, Maharashtra, Ratnagiri district, Amba Ghat, 29.08.1967, M.Y. Ansari 105001A (holo CAL!).

Fig. 24

Vernacular name: Kharpudi (Marathi).

Perennial twining herbs. Rootstock tuberous; tubers $1.8-2.6 \mathrm{~cm}$ in diam., sub-globose, roots fibrous. Stem c. $200 \mathrm{~cm}$ in long, terete, usually single from tuber, branched, glabrous. Lower lamina ovate-lanceolate, $12-13.5 \times 3.5-5 \mathrm{~cm}$, acute at apex, broad at base; upper lamina ellipticlanceolate, $7.5-8 \times 2.3-2.5 \mathrm{~cm}$, acute at apex, rounded-cordate at base, membranous, ciliolate along margins; petioles $2.7-3 \mathrm{~cm}$ long, glabrous, channeled above. Cymes sub-umbellate, manyflowered; peduncles 10.5-15 cm long, puberulous; bracts 2.5-3.5 mm long, linear, glabrous; pedicels $1.5-1.7 \mathrm{~cm}$ long, pubescent. Sepals $2.5-3 \mathrm{~mm}$ long, subulate, glabrous. Corolla $1-1.2 \mathrm{~cm}$ long, straight; tube c. $5 \mathrm{~mm}$ long, with 5 distinct ridges outside, broad at middle, glabrous, purple lines within, pinkish-white; lobes 7-8 $\mathrm{mm}$ long, $c$. $1.8 \mathrm{~cm}$ across, ovate, deeply cordate, broader than long, connate at tips and forming depressed circular cage, glabrous. Corona bi-seriate; outer of 5 lobed, cupular or saucer-shaped, entire, $c$. $0.7 \mathrm{~mm}$ long, inconspicuous, glabrous; inner of 5 elongated conical lobes, c. $0.2 \mathrm{~mm}$ long, with upwardly pointed hairs, creamy-yellow. Follicles in pairs, 6-8.5 cm long, tapering at both ends, glabrous. Seeds many, c. $5 \times 3 \mathrm{~mm}$, ovate, oblong, winged, comose; coma $c .1 \mathrm{~cm}$ long, silky white.

Flowering \& fruiting: July-October.

Chromosome number: $2 n=22$ (Raghavan \& Ansari, 1975).

Habitat: Grows along rocky hill slopes in association with Euphorbia antiquaorum L., Strobilanthes callosa Nees, Tripogon lisboae Stapf and other grasses at an elevation above $600 \mathrm{~m}$.

Distribution: Endemic to northern Western Ghats 

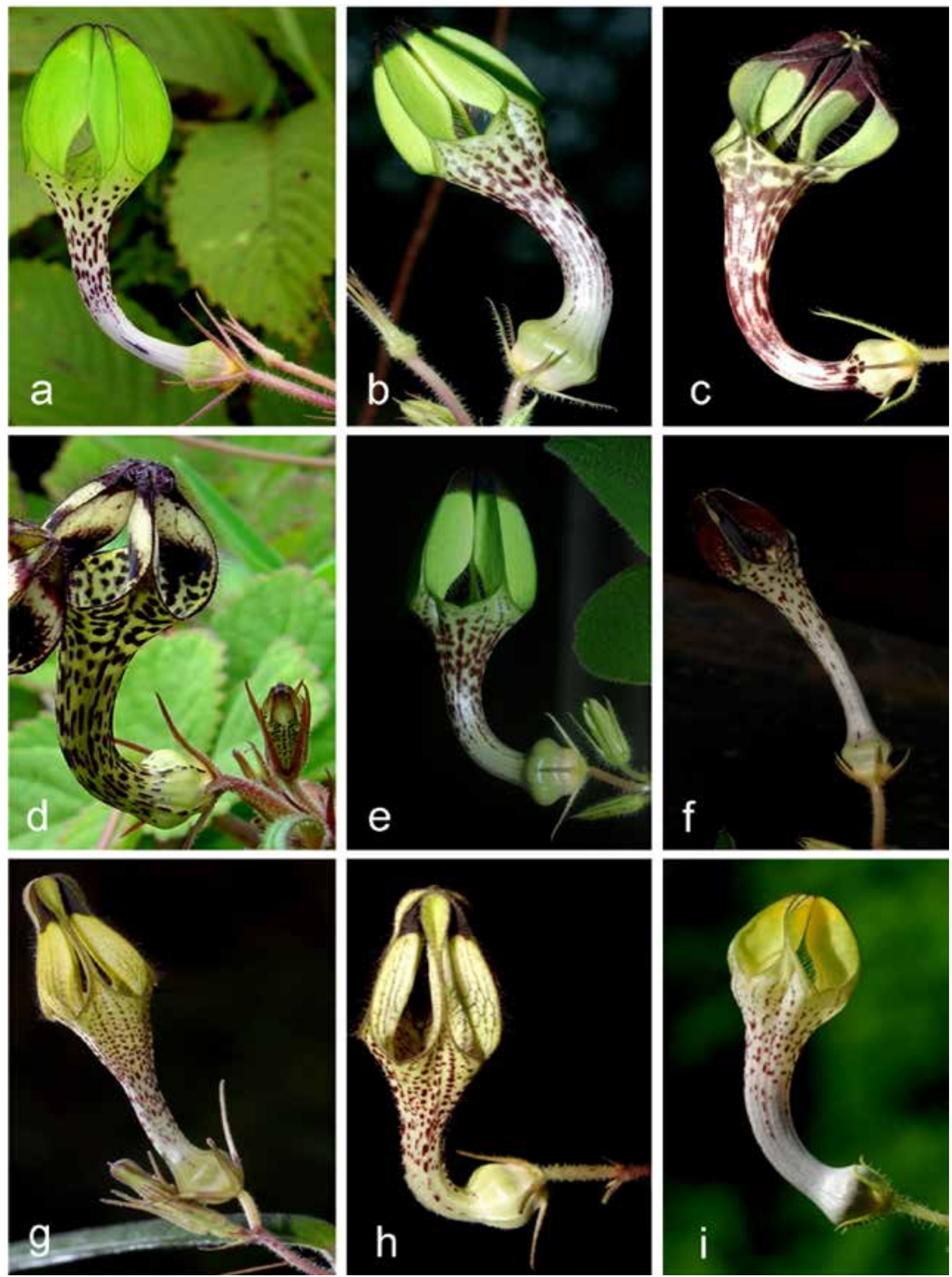

Fig. 23. Floral variations in Ceropegia hirsuta Wight \& Arn.: a. Corolla lobes green and without purple colour at the tip; $\mathbf{b}$. Corolla lobes with purple tip; c. Corolla lobes with upper 1/3rd purple tip; $\mathbf{d}$. Corolla lobes hairy and purple at the tip; e. Discoid dilated portion of the tube; $\mathbf{f}$. Corolla lobes red throughout; $\mathbf{g}$. Corolla lobes hairy throughout; $\mathbf{h}$. Corolla hairy outside; i. Corolla lobes yellow, hairy and without purple tip. 

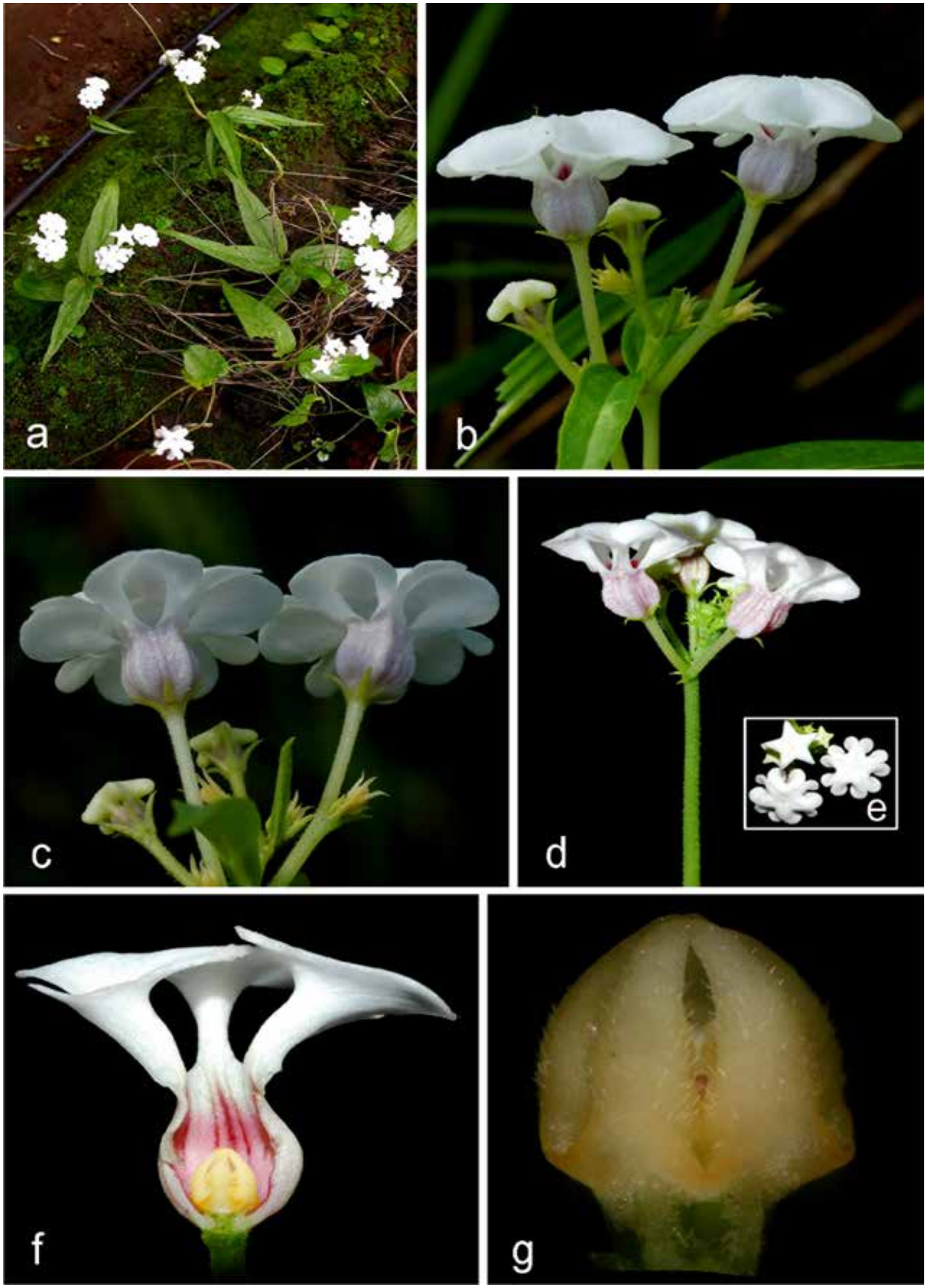

Fig. 24. Ceropegia huberi Ansari: a. Habit; b-d. Inflorescence; e. Top view of corolla lobes; f. Flower L.S.; g. Corona-lateral view. 
of Maharashtra, India.

Specimens examined: INDIA, Maharashtra, Kolhapur district, Anuskura ghat, 24.08.2014, S.S. Kambale \& S.R. Yadav SSK 285; Bhuibawda ghat, 24.08.2014, S.S. Kambale \& S.R. Yadav SSK 287; Gaganbawada, 09.1996, M.P. Bachulkar-Cholekar MPB s.n.; Ibid., 04.08.2003, M.Y. Kamble MYK s.n.; Karul Ghat, 28.07.2013, S.S. Kambale SSK 55; Ibid., 19.08.2013, S.S. Kambale \& P.R. Lawand SSK 71; Panhala, 15.07.1973 K.S. Patil P 3280; Tillari, 03.09.2012, S.S. Kambale SSK 20 (SUK!); Ratnagiri district, Amba ghat, 05.07.1997, S.R. Yadav s.n.; Ibid., 12.08.1997, P.S. Walkunde s.n.; 22.09.1998, P.D. Mahekar \& V.B. Shimpale 5823; Ibid., 25.09.1999, V.T. Patil 417; Ibid., 20.07.2003, M.Y. Kamble MYK 2115; Ibid., s.d., S.P. Gaikwad SPG 474; Ibid., (grown in garden), 30.07.2011, S.S. Kambale SUK 2588; Ibid., 15.08.2013, M.M. Lekhak SSK 70 (SUK!); Pune district, Mulashi, 24.08.2013, S.S. Kambale E S.R. Yadav SSK 57; Ibid., 24.08.2014, S.S. Kambale \& S.R. Yadav SSK 262; Varandha Ghat, 07.07.2004, M.Y. Kamble MYK s.n. (SUK!).

Conservation status: Nayar and Sastry (1987) assessed it as Vulnerable.

Notes: Ceropegia huberi is closely similar to $C$. santapaui but differs by its bright white flowers and much depressed cage of corolla lobes.

Ceropegia lawii Hook.f., Fl. Brit. India 4: 67. 1883; Blatter \& McCann, J. Bomb. Nat. Hist. Soc. 36: 534. 1933; H. Huber, Mem. Soc. Broter. 12: 67. 1957; T. Cooke, Fl. Bombay 2: 240. 1905; Ansari, Fasc. Fl. India 16: 19. 1984; M.P. Nayar \& Sastry, Red Data Book Indian Pl. 1: 60. 1987; A.P. Jagtap \& N.P. Singh, Fasc. Fl. India 24: 228. 1999; D.K. Mishra \& N.P. Singh, Endem. Threat. Pl. Maharashtra 136. 2001; A.P. Jagtap \& Das Das in N.P. Singh et al., Fl. Maharashtra Dicot. 2: 352. 2001; F. Albers \& Meve, Ill. Handb. Succ. Pl. Asclepiadaceae 85. 2002; Kambale \& S.R. Yadav, Asklepios 115: 35. 2013; Punekar et al., Nelumbo 55: 17. 2013. Lectotype (Step I designated by Huber, 1957; Step II designated by Punekar et al., 2013): INDIA, Maharashtra, Concan, s.d., Stocks \& Law Ceropegia 25 (K000357607 image!).

C. maharashtrensis Punekar, Tamhankar, Lakshmin., Kumaran, Raut \& S.K. Srivast., Nelumbo 55: 22. 2013. Type: INDIA, Maharashtra, Dhak killa (Dhakeshwar), 1100 m, 08.2005, S.A. Punekar \&

\section{A. Raut 357 (holo CAL!; iso AHMA).}

"C. lawii Hook.f. var. wadhwae" M.R. Almeida, Fl. Maharashtra 3A: 234. 2001. nom. inval.

C. sahyadrica auct. Lakshmin. \& Sharma, Fl. Nasik District 302. 1991, non Ansari \& B.G. Kulk, 1997.

Fig. 25

Vernacular names: Kharpudi, Tilori, Korpudi (Marathi).

Perennial erect herbs. Rootstock tuberous; tubers $2-4.5 \mathrm{~cm}$ in diameter, sub-globose, roots fibrous. Stem c. 45-60 cm long, usually single from individual tuber, rarely 1-3 from the same tuber, terete, sometimes quadrangular, generally unbranched, pubescent. Leaves opposite-decussate to whorls of 3-4; lamina variable, lower ovate, acute at apex, rounded-cordate at base, upper ovate-lanceolate $5-6 \times 2.8-3 \mathrm{~cm}$, puberulous above with bulbous based hairs, glabrous beneath, ciliate along margins, lateral nerves 3-5 pairs; petioles $1.5-1.7 \mathrm{~cm}$ long, hairy, channeled above. Cymes umbellate, 2-many-flowered, extra-axillary; peduncles $0.5-2 \mathrm{~cm}$ long, puberulous; bracts 1-1.5 mm long, linear; pedicels $8-9 \mathrm{~mm}$ long, puberulous. Sepals 2-2.5 mm long, linear, hairy along midnerve, glabrous otherwise. Corolla 2.5-3 cm long, straight or slightly curved; tube 1.7-1.9 $\mathrm{cm}$ long, narrow in the middle, sub-cylindrical above, minutely hairy at the base of dilated part within, greyish white, deep purple in the lower $2 / 3^{\text {rd }}$ part and white in upper $1 / 3^{\text {rd }}$ part within; lobes 8-9 $\mathrm{mm}$ long, ovate-cordate, connate at the tip forming globose-ovoid cage, 3-7 $\mathrm{mm}$ across, glabrous, variously coloured, externally greyishwhite, faint green-faint purple within. Corona bi-seriate; outer of 5 entire-bifid lobes, saucer to cupular-shaped, shorter than gynostegium, c. $2 \mathrm{~mm}$ long, hairy along the margins, yellow-deep purple with black margins; inner straight, 2.5-3 mm long, linear-spathulate, yellow-white. Follicles in pairs, 9-12 cm long, erect, tapering towards apex. Seeds c. $6 \times 2 \mathrm{~mm}$, oblong-elliptic, brown, comose; coma c. $2.5 \mathrm{~cm}$ long, silky white.

Flowering \& fruiting: July-September.

Habitat: Grows along hill slopes in association with Strobilanthes callosa Nees, at an elevation ranging from 210-1232 m.

Distribution: Endemic to northern Western Ghats 


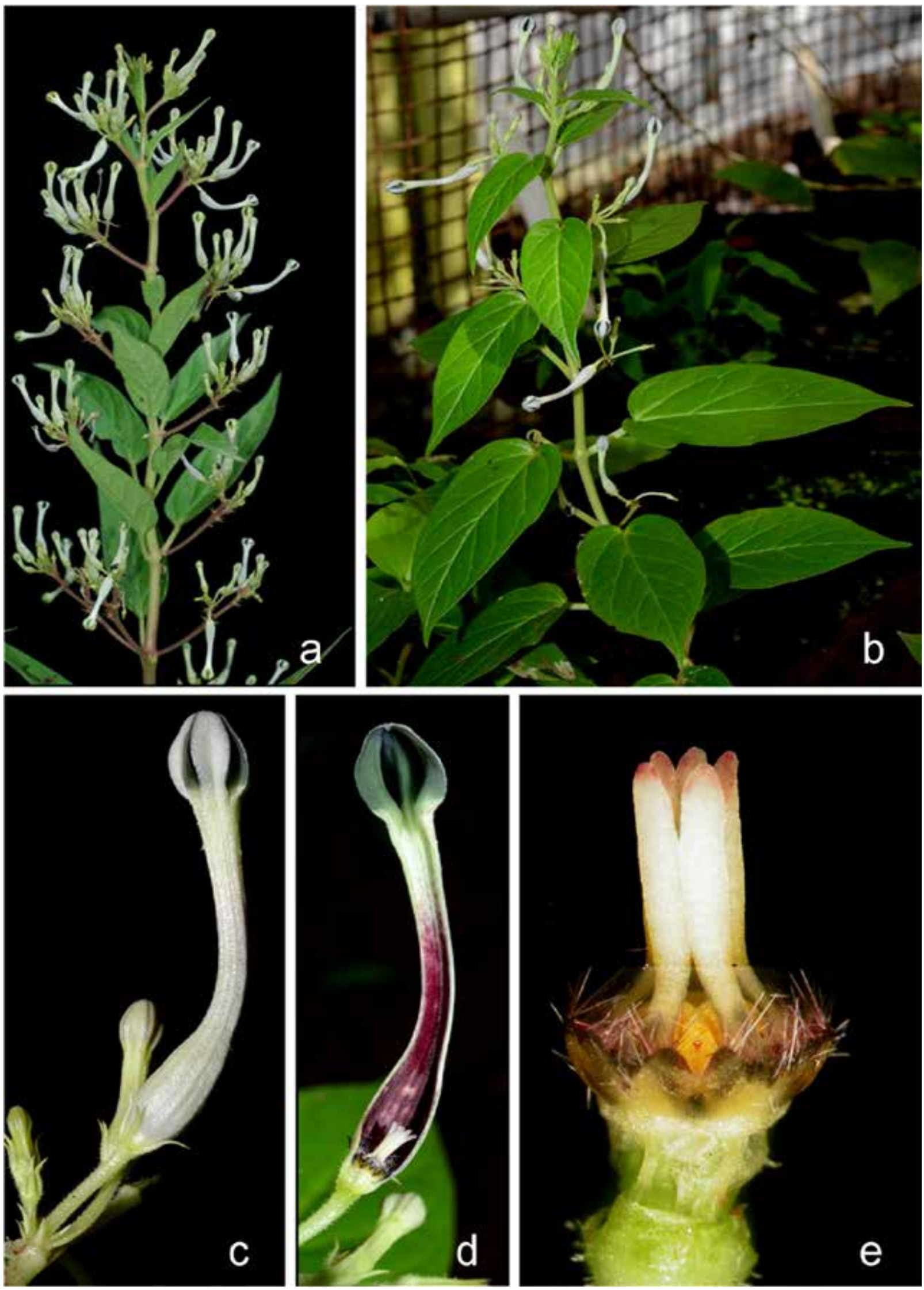

Fig. 25 Ceropegia lawii Hook.f.: a \& b. Habit; c. Flower; d. Flower L.S.; e. Corona-lateral view. 
of Maharashtra, India.

Specimens examined: INDIA, Maharashtra, Ahmednagar district, Harishchandragad, 15.08.2014, S.S. Kambale SSK 272; Ibid., 24.08.2014, S.S. Kambale \& A.N. Chandore SSK 290; Ibid., 12.09.2014, S.S. Kambale \& A.A. Adsul SSK 304; Ibid., 20.10.2014, Ibid. (grown in garden), S.S. Kambale SSK 328 (SUK!); Nasik district, Anjaneri (grown in garden), 22.06.2011, S.S. Kambale SUK 2572; Ibid., 16.07.2011, S.S. Kambale SUK 2579 (SUK!).

Conservation status: Nayar and Satry (1987) assessed it as Endangered.

Notes: According to Huber's (1957) classification of the genus, C. lawii comes under Ceropegia sect. Buprestis. This species is highly variable in its floral morphology and inflorescence pattern (Kambale \& Yadav, 2015). Inflorescence is sometimes a solitary flower to few flowered, lateral umbellate cyme. Number of inflorescence also varies from 1-4 per node. Colour of corolla lobes within upper-half varies considerably from pale-green and deep purple to yellow. It is closely allied to C. panchganiensis, C. sahyadrica, C. rollae and $C$. maccannii in having erect habit, but differs in having linear, erect, glabrous, inner corona forming a complex of these taxa. Meve (2002) synonymised C. maccannii and C. panchganiensis under C. lawii and Punekar et al. (2013) added two new species, viz. C. maharashtrensis Punekar, Tamhankar, Lakshmin., Kumaran, Raut \& S.K. Srivast. and C. karulensis Punekar, Tamhankar, Lakshmin., Kumaran, Raut, S.K. Srivast. \& Kavade. The former is recognized as a synonym while the latter is considered as a variety under C. sahyadrica Ansari \& B.G. Kulk. This taxon is distinct from the other species of the complex in having cupular, outer hirsute corona and narrow corolla tube.

Ceropegia maccannii Ansari, Bull. Bot. Surv. India 22(1-4): 227. 1980 (1982); Ansari, Fasc. Fl. India 16: 22. 1984; M.P. Nayar \& Sastry, Red Data Book Indian Pl. 2: 47. 1988; A.P. Jagtap \& N.P. Singh, Fasc. Fl. India 24: 230. 1999; D.K. Mishra \& N.P. Singh, Endem. Threat. Pl. Maharashtra 138. 2001; A.P. Jagtap \& Das Das in N.P. Singh et al., Fl. Maharashtra Dicot. 2: 352. 2001; Karthik. et al., Fl. Pl. India 1: 164. 2009; Kambale \& S.R. Yadav, Asklepios 115: 35. 2013; Punekar et al., Nelumbo
55: 21. 2013. Ceropegia lawii var. maccannii (Ansari) M.R. Almeida, Fl. Maharashtra 3A: 234. 2001. Type: INDIA, Maharashtra, Pune district, Sinhgad hill, 08.1964, M.Y. Ansari 97574A (holo CAL!; iso BSI!).

C. lawii auct. T. Cooke, Fl. Bombay 2: 175. 1904; H. Huber, Mem. Soc. Brot. 12: 67. 1957; Venkatareddi, Willdenowia 5(1): 32. 1968, non Hook.f., 1883.

Fig. 26

\section{Vernacular name: Kharpudi (Marathi).}

Perennial erect herbs. Rootstock tuberous; tubers $2-6 \mathrm{~cm}$ in diam., sub-globose, roots fibrous. Stem 40-180 cm long, usually single from each tuber, rarely two, terete, usually unbranched, rarely branched, pubescent, pale purple. Lamina variable, lower ones 7.2-9.2 × 2.6-3.5 cm, ovate-lanceolate, acute-acuminate at apex, round-cordate at base, pubescent above, with bulbous based hairs, glabrous beneath except along nerves and midrib, usually 2-3 glands at the base of lamina; upper ones linearlanceolate rarely falcate, $1.7-6.2 \times 0.25-1.6 \mathrm{~cm}$, with 2 glands at the base, ciliolate along margins; petioles $0.2-1.9 \mathrm{~cm}$ long, channeled above, pubescent. Cymes sub-umbellate, 3-5-flowered, extra-axillary; peduncles 0.4-1.6 cm, slender, puberulous; bracts 3-4 mm long, linear, glabrous; pedicels 3-6 mm long, slender, pubescent. Sepals 4-4.2 mm long, linear-lanceolate, puberulous along midnerve, glabrous otherwise. Corolla 2-2.1 cm long, greyish-yellow; tube 1.6-1.8 cm long, straight-slightly curved, gradually dilated towards base, light windows at the dilated part within, glabrous within, purple; lobes c. $3 \mathrm{~mm}$, obovate, acute at apex, connate at tips forming a minute obovate or obconic cage of 3-3.5 mm across, glabrous, yellow. Corona bi-seriate; outer of 5 entire triangular lobes, cupular, c. $2 \mathrm{~mm}$ long, hairy almost throughout and within; inner thick, fleshy, sub-clavate to clavate, $2 \mathrm{~mm}$ long, hairy, usually white rarely with purple blotching. Follicles in pairs, 4.5-6.5 cm long, linear, terete, tapering at the ends, glabrous. Seeds $5-6 \times 2-3 \mathrm{~mm}$, ovate, oblong, marginate, comose; coma 1.5-2 cm long, silky white.

Flowering \& fruiting: May-August.

Chromosome number: $2 n=22$ (Raghavan \& Ansari, 1975).

Habitat: Grows on hill slopes, in association, with Strobilanthes callosa Nees and Rhinacanthus sp. at an 


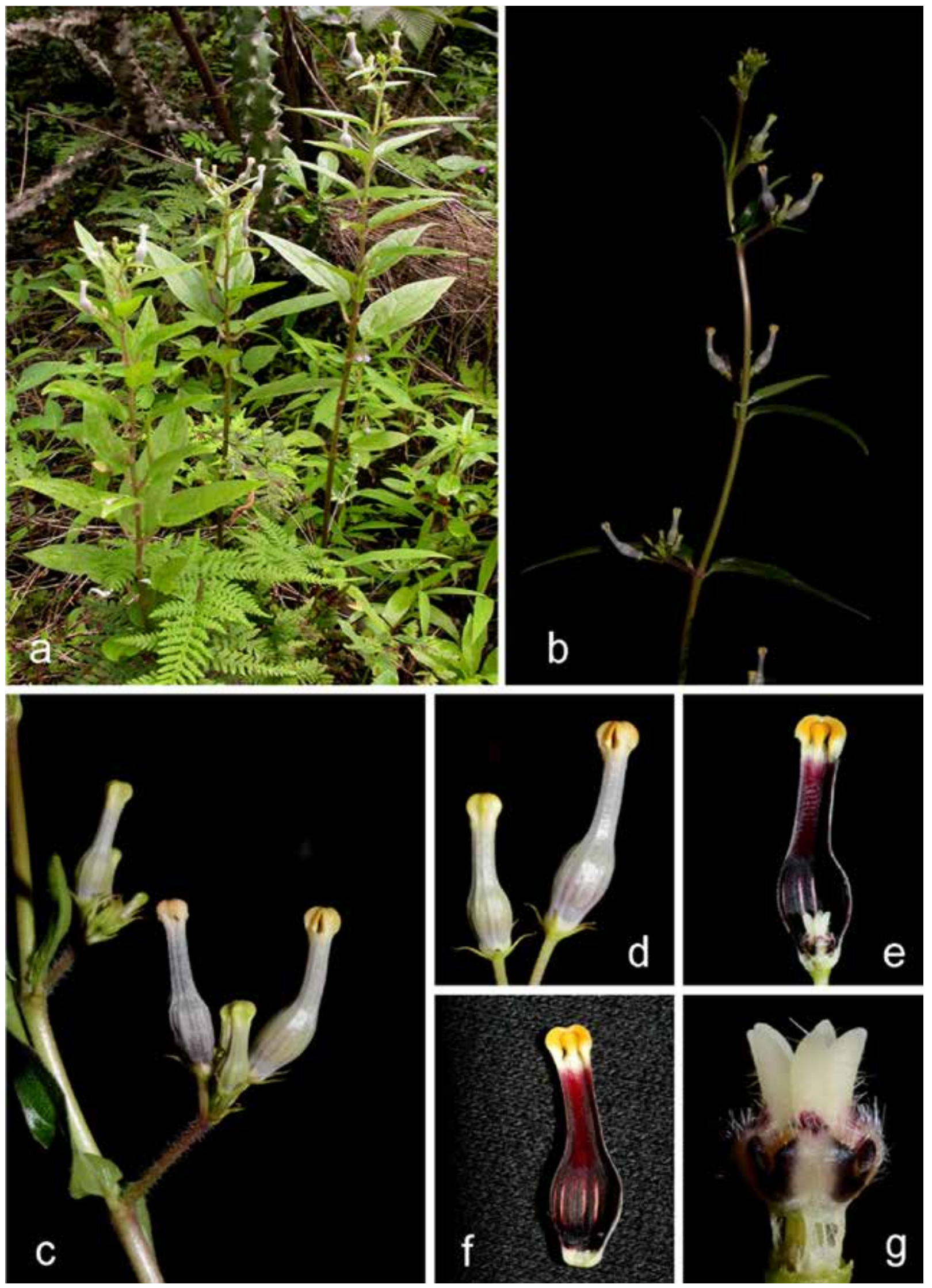

Fig. 26. Ceropegia maccannii Ansari: a \& b. Habit; c. Inflorescence; d. Flowers; e. Flower L.S.; f. Corolla tube cut open; g. Corona-lateral view. 
elevation of 943-1000 m.

Distribution: Endemic to northern Western Ghats of Maharashtra, India.

Specimens examined: INDIA, Maharashtra, Pune district, Purandhar fort, 08.1991, s.coll. s.n. (MH!); Sinhgadh, 29.08.1963, M.Y. Ansari 87804 (BSI!); Ibid., 07.1965, B.V. Reddi 101207 (CAL!); Torna Fort (grown in garden), 26.08.2010, S.S. Kambale SUK 2558; Ibid., 16.07.2011, S.S. Kambale SUK 2582; Ibid., 19.09.2012, S.S. Kambale SSK 21; Ibid., 12.08.2013, S.S. Kambale SSK 68; Ibid., 06.09.2013, S.S. Kambale SSK 74; Ibid., 15.08.2014, S.S. Kambale SSK 273; Ibid., 21.08.2014, S.S. Kambale SSK 284 (SUK!); Varandha Ghat, 15.08.2014, S.S. Kambale SSK 271 (SUK!).

Conservation status: Nayar and Sastry (1987) assessed it as Rare. This plant is represented by very few individuals in its area of occurrence due to the habitat loss and exploitation of tubers for food. In the present study, it is assessed as Critically Endangered [CR: B2 a, b (iii, iv, v)].

Notes: Ceropegia maccannii is morphologically related to C. panchganiensis Blatt. \& McCann in having clavate inner corona; but it differs in having relatively short flowers, shorter corolla lobes and ovate-lanceolate leaves. C. maccannii is often confused with C. lawii due to its morphological similarities. Specimens of this taxon were collected as early as in 1833 by Law and Stocks, who identified it as C. lawii. Hooker (1833) and Venkatareddi (1968) also misidentified it as the latter. However Ansari (1984) described it as new and can be distinguished by smallest flowers and narrow corolla tube, hence treated here as distinct.

Ceropegia media (H. Huber) Ansari, Bull. Bot. Surv. India 11(1-2): 199. 1971; Ansari, Fasc. Fl. India 16: 24. 1984; A.P. Jagtap \& N.P. Singh, Fasc. Fl. India 24: 232. 1999; D.K. Mishra \& N.P. Singh, Endem. Threat. Pl. Maharashtra 131. 2001; A.P. Jagtap \& Das Das in N.P. Singh et al., Fl. Maharashtra, Dicot. 2: 353. 2001; M.R. Almeida, Fl. Maharashtra 3A: 235. 2001; F. Albers \& Meve, Ill. Handb. Succ. Pl. Asclepiadaceae 89. 2002; Karthik. et al., Fl. Pl. India 1: 162. 2009. Ceropegia evansii McCann var. media H. Huber, Mem Soc. Brot. 12: 67. 1957. Type: INDIA, Maharashtra, Pune district, Bhimashankar hills, N.A. Irani 1194 (holo BLAT image!).

Fig. 27

Perennial twining herbs. Rootstock tuberous, tubers $1-4 \mathrm{~cm}$ in diam., globose or sub-globose, roots fibrous. Stem c. $3 \mathrm{~m}$ long, usually single from individual tuber, terete, unbranched, green to dull purple, glabrous. Lamina 6-16 × 1.3-4.5 $\mathrm{cm}$, sub-coriaceous, linear-lanceolate to ellipticlanceolate, acute-acuminate, narrowed at base, ciliolate along margins and midnerve beneath, glabrous beneath; petioles $0.5-2.4 \mathrm{~cm}$ long, channeled above, hairy along margins, glabrous otherwise. Cymes umbellate, 3-8-flowered, extra-axillary; peduncles $1-2.8 \mathrm{~cm}$ long, terete, hirsute; bracts 4-7.5 mm long, linear-subulate, glabrous, slightly hairy along margins; bracteoles 2-2.5 mm long, linear-lanceolate, sparsely hairy along margins; pedicels $0.5-1 \mathrm{~cm}$ long, slender, hirsute. Sepals c. $5 \mathrm{~mm}$ long, glabrous otherwise, linear-lanceolate, subulate, hairy along midnerve, glabrous otherwise. Corolla $2.1-2.5 \mathrm{~cm}$ long; tube $1.4-1.8 \mathrm{~cm}$, slightly curved, abruptly dilated at base, cylindrical at middle, funnel-shaped at throat, glabrous within and outside, pale-purple to white outside, pale-green at dilated part and deep purple in the remaining part within, white at throat; lobes slightly reflexed, oblong-spathulate, connate at tip forming obovoid-globose cage, glabrous, pale pink-purple. Corona stipitate, bi-seriate; outer of 5-bifid emarginate lobes, cupular, sub-quadrate in outline, 2-2.5 mm long, usually glabrous outside, hairy within, fleshy dark purple with white tinge; inner of 5 linear lobes, 1-2 mm long, white-cream coloured. Follicles in pairs usually unequal, hooked at tip, 9.5-11.4 cm long, tapering at both ends, glabrous. Seeds $6 \times 2.5 \mathrm{~mm}$, comose; coma silky white.

Flowering \& fruiting: July-September.

Chromosome number: $2 n=22$ (Raghavan \& Ansari, 1975).

Habitat: Grows along hills slopes in association with Strobilanthes callosa Nees. It also found in forest edges along with Memecylon umbellatum Burm.f.

Distribution: Endemic to northern Western Ghats of Maharashtra, India.

Specimens examined: INDIA, Maharashtra, Ahmednagar district, Kalsubai, 02.09.2003, M.Y. Kamble 2151 (SUK!); Pune district, Donaje, Dhorjai 

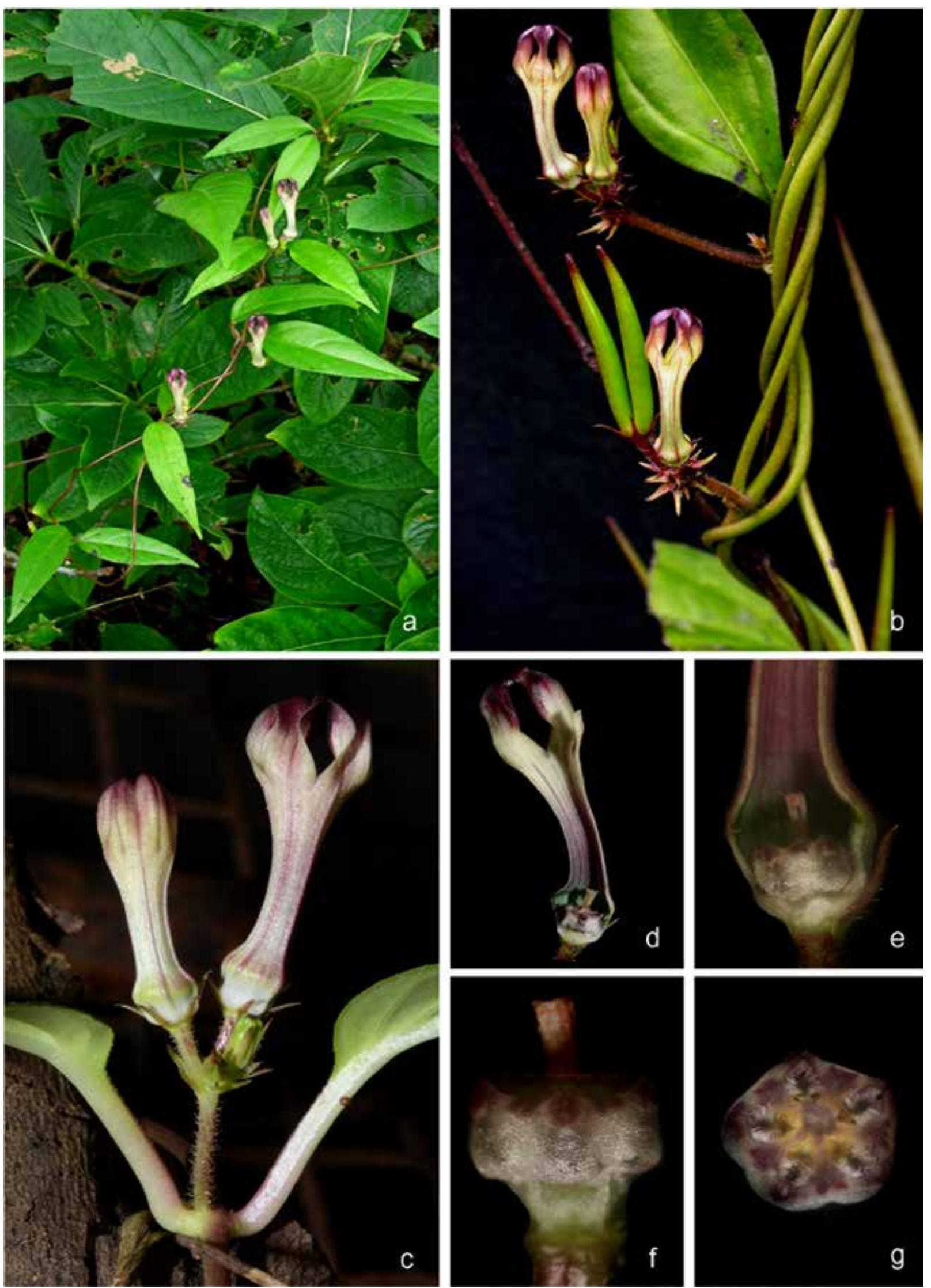

Fig. 27. Ceropegia media (H. Huber) Ansari: a \& b. Habit; c. Flowers; d \& e. Flower L.S.; f. Corona-lateral view; g. Corona-top view. 
Forest, 07.08.1964, M.Y. Ansari 97555; Khanapur, Sinhgadh hill slopes, 12.08.1964, M.Y. Ansari 99835 (BSI!); Varandha Ghat, 15.07.2003, M.Y. Kamble s.n. (SUK!); Ratnagiri district, Gothane, 24.08.2014, S.S. Kambale SSK 292 (SUK!); Satara district, Kas, 09.1994, M.P. Bachulkar-Cholekar 5806; 10.1995, M.P. Bachulkar-Cholekar 20660; 25.07.2010, S.S. Kambale SUK 2569; 31.07.2011, S.S. Kambale SUK 2587; Ibid. (grown in garden), 30.08.2012, S.S. Kambale SSK 17 (SUK!); Thoseghar plateau, 29.07.2002, S.P. Gaikwad SPG 212; 14.08.2004, M.Y. Kamble s.n. (SUK!).

Conservation status: Observations on populations at various localities in northern Western Ghats of Maharashtra revealed that the species represented by a few individuals at each locality. Mishra and Singh, 2011 categorized it as Vulnerable, and is supported in the present study.

Notes: Huber (1957) described this taxon as a variety of C. evansii McCann, based on the collections of Irani (Irani 1194) housed at BLAT. Subsequently, Ansari (1984) elevated the variety to the rank of a species. Ceropegia media is similar to C. evansii and C. omissa, however, it is distinguished by its linear to lanceolate leaves, puberulous pedicels and calyx, straight to slightly curved corolla, cupular to sub-quadrate outer corona and inner corona with linear lobes.

Ceropegia oculata Hook., Bot. Mag. 70: t. 4093. 1844; Dalzell \& A. Gibson, Bombay Fl. 154. 1861; Hook.f., Fl. Brit. India 4: 72. 1883; T. Cooke, Fl. Bombay 2: 242. 1905; M.P. Nayar \& Sastry, Red Data Book Indian Pl. 1: 62. 1987; S.M. Almeida, Fl. Savantwadi 1: 258. 1989; S.D. Deshp. et al., Fl. Mahabaleshwar, Maharashtra 1: 361. 1993; A.P. Jagtap \& N.P. Singh, Fasc. Fl. India 24: 234. 1999; D.K. Mishra \& N.P. Singh, Endem. Threat. Pl. Maharashtra 144. 2001; A.P. Jagtap \& Das Das in N.P. Singh et al., Fl. Maharashtra, Dicot. 2: 354. 2001; M.R. Almeida, Fl. Maharashtra 3A: 236. 2001; F. Albers \& Meve, Ill. Handb. Succ. Pl. Asclepiadaceae 93. 2002; Karthik. et al., Fl. Pl. India 1: 163. 2009; Kambale \& S.R. Yadav, Asklepios 115: 37. 2013. Lectotype (designated by Ansari, 1984): Botanical Magazine, t. 4093. 1844.

Ceropegia oculata Hook. var. satpudensis Punekar, S.D. Jagtap \& Deokule, Curr. Sci. 91(9): 1143. 2006. Type: INDIA, Maharashtra, Nandurbar district, Akrani taluka, Toranmal, Legapani village, 09.08.2003, S.D. Jagtap 2919 (holo CAL!; iso BSI!).

Ceropegia mahabalei Hemadri \& Ansari var. hemalatae S.S. Rahangdale \& S.R. Rahangdale, Indian Forester 138(2): 201. 2012. Type: INDIA, Maharashtra, Pune district, Junnar taluka, Shindechiwadi, S.R. Rahangdale 0137 (holo BSI!).

Fig. 28

Vernacular names: Kharpudi, Khapar-khutti (Marathi).

Perennial twining herbs. Rootstock tuberous; tubers c. $5 \times 2.5 \mathrm{~cm}$, globose; roots fibrous. Stem sparingly branched, terete, slightly swollen at the nodes, c. 3 $\mathrm{m}$ long. Lamina $4-19 \times 1.7-11.5 \mathrm{~cm}$, broadly ovate to ovate-oblong, acute or shortly acuminate at apex, rounded-cordate at base, sparsely pubescent with bulbous based hairs above, glabrous or hairy on the nerves beneath, glabrous otherwise; petioles 0.6-5.4 (-8) cm long, channeled above, hairy along margins, glabrous otherwise. Cymes umbellate, 6-11-flowered, extra-axillary; peduncles 1.9-4.4 cm long, terete, hirsute; bracts solitary, 4-9 × 1-1.5 $\mathrm{mm}$, linear-subulate or lanceolate, glabrous; pedicels 0.9-2.5 cm long, slender, glabrous. Sepals 0.65-1.3 $\mathrm{cm}$, linear-subulate, glabrous, sometimes hairy along midnerve, whitish-green to reddish-purple. Corolla 5.8-7.8 cm long; tube 4.7-5.6 cm long, curved in $90^{\circ}$ with axis, gradually-abruptly dilated at the base, narrow in the middle, widening into the funnel-shaped throat, whitish-green to faint purple at the dilation and blotched with dark red spots at the mouth, greyish-white with purple striations in the middle; lobes $1.5-2.0 \mathrm{~cm}$ long, linear-obtuse, connate at tip to form narrow beak, glabrous, ciliate within above the middle and along margins; downwardly directed hairs within lower half, green at upper-half, white tinged with green at lower half, sometimes purple throughout (colour of corolla lobes is variable; usually deep green above, white at middle and deep purple below). Corona stipitate, biseriate; outer of 5-bifid lobes, saucer-shaped, with 3-4 mm long, sparsely ciliate along margins and within, yellow to deep purple; inner 4-5 mm long, erecto-divergent, linear-clavate, glabrous, yellow, deep purple at base. Follicles in pairs, horizontal, $c$. $12.5 \mathrm{~cm}$ long, glabrous. Seeds $c .7 \times 3 \mathrm{~mm}$, comose; coma c. $2.5 \mathrm{~cm}$ long.

Flowering \& fruiting: July-October.

Chromosome number: $2 n=22$ (Raghavan \& Ansari, 

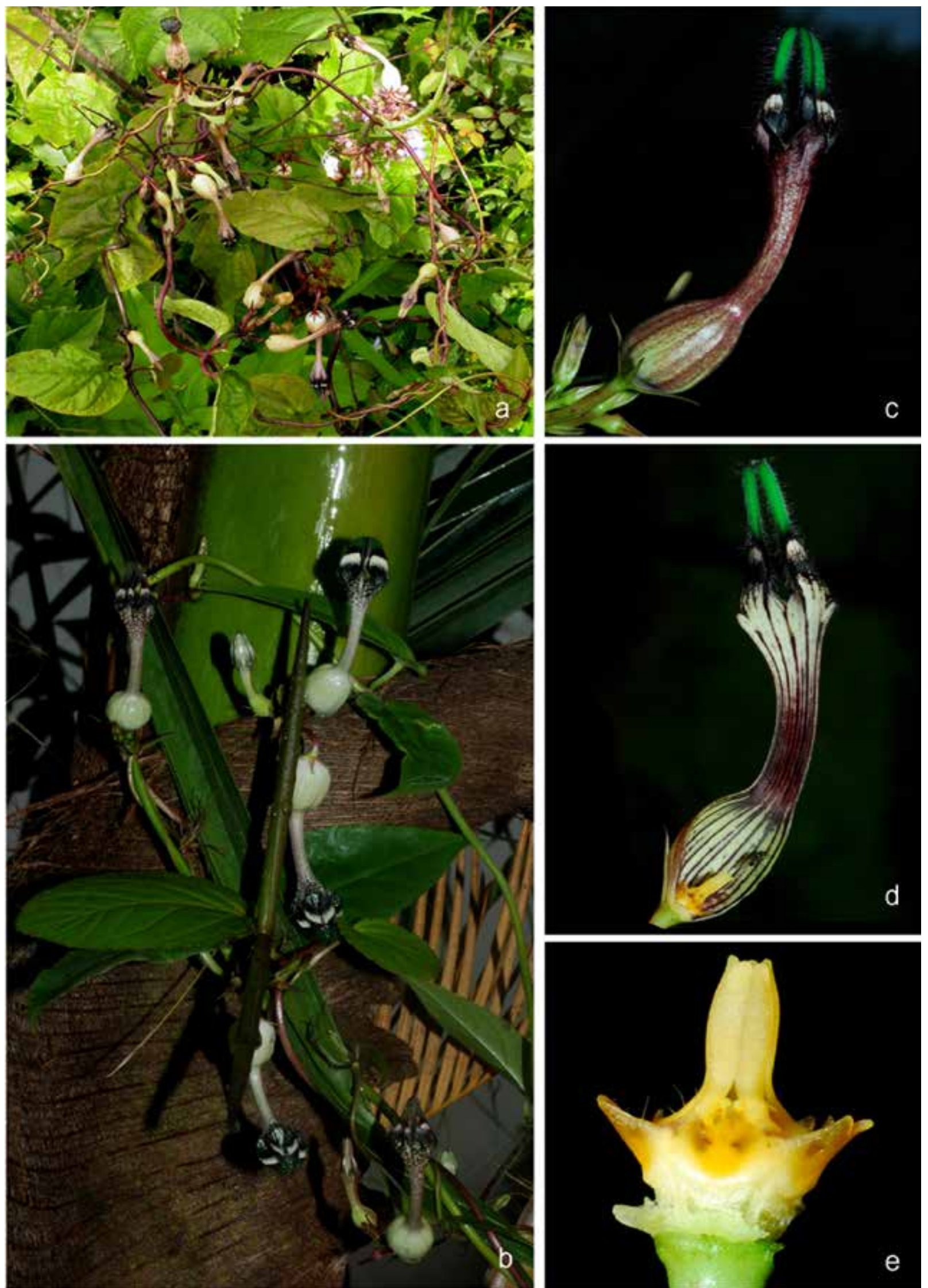

Fig. 28. Ceropegia oculata Hook.: a \& b. Habit; c. Flower; d. Flower L.S.; e. Corona-lateral view. 
1975).

Habitat: Grows amidst bushes, along hills slopes in association with Strobilathes callosa Nees, Bridelia retusa etc.

Distribution: Endemic to Western Ghats (Karnataka, Kerala, Maharashtra), India.

Specimens examined: INDIA, Maharashtra, Kolhapur district, Panhala, s.d., M.M. Sardesai 203; Ibid., Masai Plateau, 30.08.2012, S.S. Kambale SSK 14; Ibid., 13.07.2014, S.S. Kambale SSK 240; Patgaon, Shengaon, 24.07.2011, S.S. Kambale SUK 2585; Ibid., 16.07.2011, S.S. Kambale SUK 2580; Radhanagari, 07.08.2003, M.Y. Kamble 2134 (SUK!); Pune district, Bhor, Dharjai forest, 07.08.1964, M.Y. Ansari 97572; Jaitapur, 14.08.2014, A.V.Mohite SSK 264; Junnar, Bhivade Khurd, 02.10.1965, K. Hemadri 107574; Ibid., 21.09.1968, K. Hemadri 117939; Katraj, 08.1956, G.S. Puri BSI 2965; Malshej Ghat, 23.08.2004, M.Y. Kamble s.n.; Mulashi, 09.08.2013, S.S. Kambale SSK 62 (SUK!); Purandar, 20.07.1963, S.R. Rao 88651 (BSI!); Rajgad (grown in garden), 30.08.2012, S.S. Kambale SSK 18 (SUK!); Ratnagiri district, Dukawad, Hanuman Ghat, 07.06.1970, B.G. Kulkarni 121145 (BSI!); Sinhgadh, Tanki-dara, 10.09.1964, M.Y. Ansari 101569 (BSI!); Satara district, Yewateshwar, 09.1992, M.P. BachulkarCholekar 5325; Sindhudurg district, Phonda, 05.08.2012, S.S. Kambale SSK 7; 21.08.2014, S.S. Kambale SSK 283; Salwa Dongar, s.d., S.P. Gaikwad 485; s.loc. 09.07.1999, S.R. Yadav s.n. (SUK!).

Conservation status: Nayar and Sastry (1987) assessed it as Rare while Mishra and Singh 2011 considered it as a low risk. It is represented by a good number of plants in all localities. However, food value of tubers, destruction of habitats are the major threats in near future. In present study, $C$. oculata is assessed as Near Threatened (NT).

Notes: Ceropegia oculata is an exceptionally variable species in its floral and vegetative characters. Petiole length varies from $0.6-8 \mathrm{~cm}$; leaf shape from elliptic-lanceolate to ovate-lanceolate, corolla colour from whitish-green to reddish-purple and shape of corolla tube dilation from abruptly to gradually dilated. Ceropegia oculata is closely similar to C. fantastica, C. vincifolia, C. metziana and $C$. hirsuta in vegetative stage, but differs from them in having greenish-flowers, abruptly to gradually dilated corolla tube and horizontal follicles.

Ceropegia odorata Nimmo ex J. Graham, Cat. Pl. Bombay 118.1839; Hook.f., Fl. Brit. Ind. 4: 75. 1883; Sabnis \& Bedi, Kew Bull. 25 (1): 57. 1971; Ansari, Fasc. Fl. India 16: 26. 1984; M.P. Nayar \& Sastry, Red Data Book Indian Pl. 1: 64. 1987; D.K. Mishra \& N.P. Singh, Endem. Threat. Pl. Maharashtra 145. 2001; A.P. Jagtap \& Das Das in N.P. Singh et al., Fl. Maharashtra, Dicot. 2: 354. 2001; A.P. Jagtap \& N.P. Singh, Fasc. Fl. India 24: 234. 1999; M.R. Almeida, Fl. Maharashtra 3A: 236. 2001; F. Albers \& Meve, Ill. Handb. Succ. Pl. Asclepiadaceae 93. 2002; Karthik. et al., Fl. Pl. India 1: 163. 2009; Kambale \& S.R. Yadav, Asklepios 115: 37. 2013. Neotype (designated by Singh, 2014): INDIA, Maharashtra, Salsette Island (Mumbai), Concan (Konkan), 11.1847, Stocks E J.S. Law 239 K (K000894261 image!).

Ceropegia blatteri McCann, J. Bombay Nat. Hist. Soc. 45: 210. 1945. Type: Not located or apparently none preserved.

Fig. 29

Vernacular name: Sulati-khutti (Marathi).

Perennial twining herbs. Rootstock tuberous; tubers $1-2.5 \mathrm{~cm}$ in diam., depressed, subglobose; roots fibrous. Stem slender, $c$. $2 \mathrm{~m}$ long, unbranched, sparsely hairy. Leaves whorled at young stage, usually 3 at lower node, opposite at upper nodes. Lamina 6-12.5 × 1.1$2.6 \mathrm{~cm}$, fleshy at juvenile stage, acute-acuminate at apex, narrowed at base, pubescent above and along margins, ciliolate along midrib and nerves beneath, glabrous otherwise; upper ones elliptic-lanceolate; petioles $0.4-0.6 \mathrm{~cm}$ long, channeled, hairy along margins, glabrous otherwise; lower ones large, broadly lanceolate, sub-sessile. Cymes umbellate, 6-10-flowered, extra-axillary; peduncles 0.5-2 $\mathrm{cm}$ long, terete, hirsute. Flowers yellow and sweet scented; bracts 3.5-5.5 mm long, linear, glabrous; pedicels c. $5 \mathrm{~mm}$ long, glabrous. Sepals 5.5-6 $\mathrm{mm}$ long, linear-acuminate, hairy on mid nerve. Corolla c. $3.2 \mathrm{~cm}$ long, tube $c .1 .6 \mathrm{~cm}$ long, slightly curved, gradually dilated at base, glabrous, yellow with purple lines and hairy within dilated part; $1-1.3 \mathrm{~cm}$ long, narrow, slightly reflexed, connate at tips forming an ovoid cage, glabrous, yellow. Corona bi-seriate; outer of entire lobes, c. $1.2 \mathrm{~mm}$ long; inner of 5 elongated, linear-oblong, subulate, 2-3 mm long processes, pubescent. Pollinia ovate- 
oblong. Follicles c. $10 \mathrm{~cm}$ long, tapering towards apex. Seeds c. $4 \times 2 \mathrm{~mm}$, brown, ovate, comose; coma c. $3.5 \mathrm{~cm}$ long.

Flowering \& fruiting: August-October.

Habitat: Grows in rocky areas amidst grasses and around bushes in association with Chlorophytum tuberosum (Roxb.) Baker, Dendrocalamus strictus (Roxb.) Nees, Terminalia elliptica Willd., Tylophora fasciculata Buch.-Ham. ex Wight and Wrightia tinctoria R.Br.

Distribution: India.
Specimens examined: INDIA, Madhya Pradesh, Burhanpur district, Nepanagar, M. Shaikh 4501 and M. Shaikh 4561 (SUK!). Maharashtra, Thane district, Karjat, 03.09.2011, S.S. Kambale \& S.R. Yadav SUK 2597; Kasara Ghat, s.d., S.S. Kambale E S.R. Yadav s.n. (SUK!). Rajasthan, s.loc., s.d., A. Powlett s.n. (CAL!).

Conservation status: Singh et al. (2014) treated it as Critically Endangered. This plant is not much represented in any of the Indian herbaria. During field surveys, only 50 individuals were located. It has recently been reported from Sabarkantha district
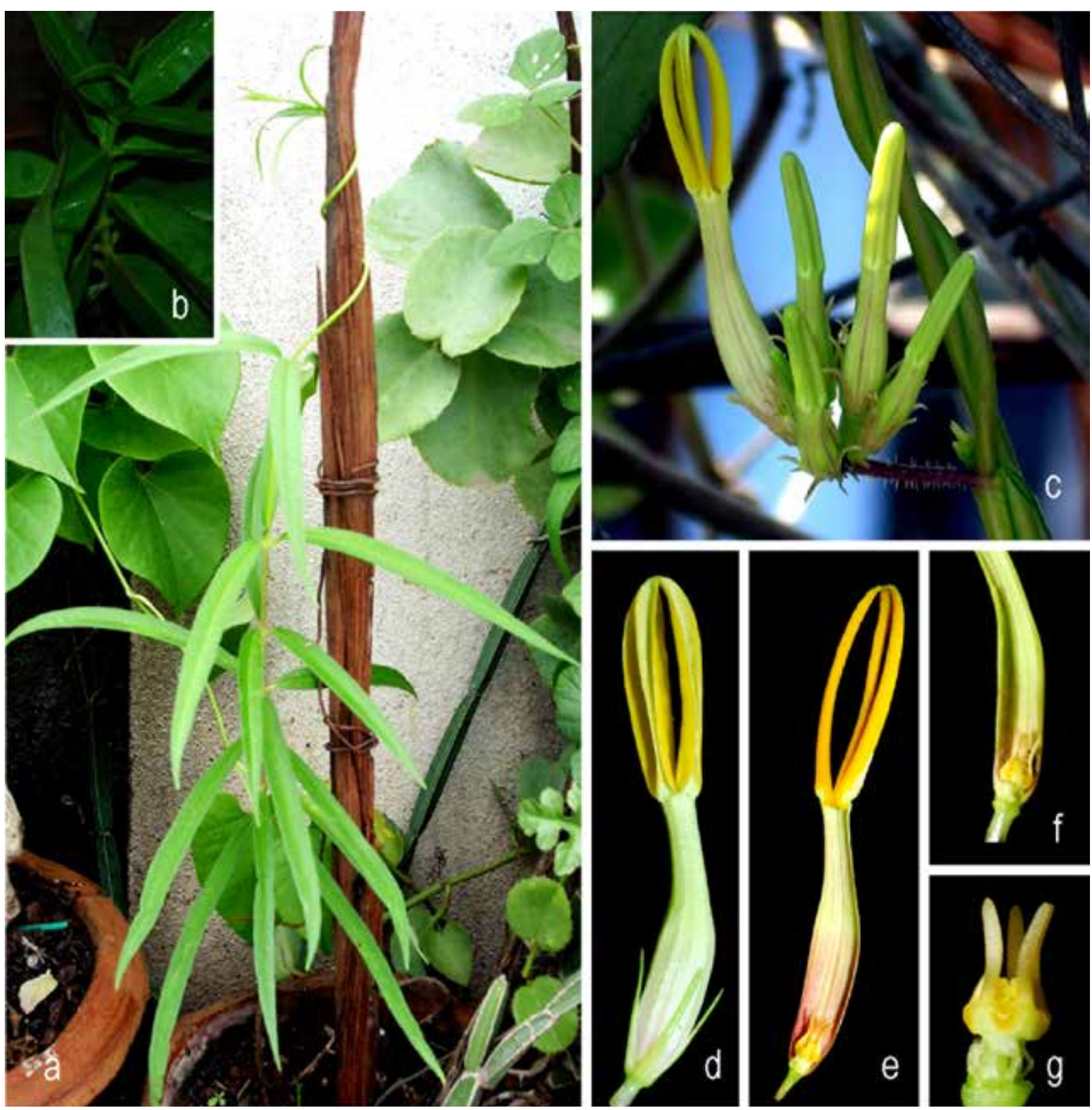

Fig. 29. Ceropegia odorata Nimmo ex J. Graham: a \& b. Habit; c. Inflorescence; d. Flower; e \& f. Flower L.S.; g. Corona-lateral view. 
of Gujarat by Patel et al. (2017). Its occurrence in Madhya Pradesh proves its wide distribution from Western Ghats to Central India. Hence, it is assessed as Vulnerable [VN: B2 a, b (iii, iv, v)] in the present study.

Notes: Ceropegia odorata is similar to C. ensifolia, but differs in having yellow ( $v s$. pale green) and shorter corolla lobes ( $v s$. as long as tube). Sweet scented flowers of this species are unusual in otherwise non-fragrant genus.

Ceropegia omissa H. Huber, Mem. Soc. Brot. 12: 67. 1957; Ansari, Fasc. Fl. India 16: 27. 1984; M.P. Nayar \& Sastry, Red Data Book Indian Pl. 1: 65. 1987; S.R. Sriniv. in Henry et al., Fl. Tamil Nadu Ind., Ser I: Analysis 2: 83. 1987; A.P. Jagtap \& N.P. Singh, Fasc. Fl. India 24: 235. 1999; T.S. Nayar et al., Fl. Pl. Kerala 86. 2006; Karthik. et al., Fl. Pl. India 1: 163. 2009. Ceropegia intermedia var. wightii Hook.f., Fl. Brit. India 4: 71. 1883; Gamble, Fl. Madras 4: 858. 1921. Lectotype (designated by Kambale \& Yadav, 2015): INDIA, Travancore, Courtallum, 1835, R. Wight s.n. (K000894272 image!).

Fig. 30

Perennial twining herbs. Lamina $3-8 \mathrm{~cm} \times 0.7-$ $2.5 \mathrm{~cm}$, ovate-lanceolate to elliptic-lanceolate, acute-acuminate at apex, rounded at base, glabrous; petioles 1-2.2 cm long, glabrous. Cymes 4-11-flowered, extra-axillary; peduncles $1-2.7 \mathrm{~cm}$ long, glabrous; pedicels c. $1.5 \mathrm{~cm}$ long, glabrous. Sepals 4-5 mm long, subulate, glabrous. Corolla $2.8-3.5 \mathrm{~cm}$ long; tube $1.5-2.5 \mathrm{~cm}$ long, slightly curved, dilated more than half of its length, subcylindrical at throat with a ring of hairs; lobes 4-10 $\mathrm{mm}$ long, ovate, connate at tips forming an ovoid cage, glabrous throughout. Corona bi-seriate; outer of 5-bifid, saucer-shaped, deltoid-linear, ciliate lobes; inner erect, linear, glabrous. Follicles 10.5-28 cm long.

Flowering \& fruiting: July-January.

Habitat: Occurs along shady places in forests.

Distribution: Endemic to Kerala and Tamil Nadu, India.

Specimens examined: INDIA, Kerala, Idukki district, Kulamavu MPCA, 24.11.1991, K. Ravikumar, P.S. Udayan \& C.R. Jawahar 14297 (FRLH!). Tamil Nadu, Tirunelveli district, Kollar forest, way to Pothagai, 16.07.1989, R. Gopalan 90689;
Kaliankattu Nerappu Parai, 29.01.1991, R. Gopalan 91714; way to Naterikal, 22.09.1916, s.coll. 33369; Sengalteri, 24.09.1916, J.S. Gamble 13540 (MH!).

Conservation status: Nayar and Sastry (1987) assessed it as Endangered while Prasad et al. (2017) assessed it as Critically Endangered. The present study also assess C. omissa as Critically Endangered [CR: B1 B2 a, b (iii)].

Notes: Hooker (1883) described C. intermedia var. wightii based on specimens collected by Wight from Courtallum in Tamil Nadu. Huber (1957) elevated this taxon to the rank of species and gave a new epithet $C$. omissa since C. wightii was preoccupied. Kambale and Yadav (2015) discussed the typification in detail and designated a lectotype. This taxon closely resembles C. intermedia in its twining habit but distinguished by its linear inner corona and glabrous outer corona.

Ceropegia panchganiensis Blatt. \& McCann, J. Bombay Nat. Hist. Soc. 36. 534. 1933; Ansari, Fasc. Fl. India 16: 27. 1984; M.P. Nayar \& Sastry, Red Data Book Indian Pl. 1: 66. 1987; S.D. Deshp. et al., Fl. Mahabaleshwar, Maharashtra 1: 362. 1993; A.P. Jagtap \& N.P. Singh, Fasc. Fl. India 24: 235. 1999; A.P. Jagtap \& Das Das in N.P. Singh et al., Fl. Maharashtra, Dicot. 2: 355. 2001; D.K. Mishra \& N.P. Singh, Endem. Threat. Fl. Pl. Maharashtra 146. 2001; Karthik. et al., Fl. Pl. India 1: 163. 2009; Kambale \& S.R. Yadav, Asklepios 115: 37. 2013; Punekar et al., Nelumbo 55: 24. 2013. Ceropegia lawii Hook.f. var. panchganiensis (Blatt. \& McCann) M.R. Almeida, Fl. Maharashtra 3A: 234. 2001. Neotype (designated by Ansari, 1984): INDIA, Maharashtra, Satara district, Lingmala near Mahabaleshwar, 20.08.1970, M.Y. Ansari 105090A (CAL!).

Ceropegia lawii auct. F. Albers \& Meve, Ill. Handb. Succ. Pl. Asclepiadaceae 85. 2002., p.p. non Hook.f., 1883.

Fig. 31

Perennial erect herbs. Rootstock tuberous; tubers $6.5-12 \mathrm{~cm}$ in diam., sub-globose; roots fibrous. Stem 14-80 (-120) cm long, generally 1 or rarely 3 , usually cylindrical, quadrangular at base, unbranched, pubescent. Leaves rarely in whorls of 3; lamina 3.5-6.3 × 2.2-3 cm, ovateoblong or ovate-lanceolate, acute-acuminate at apex, rounded-sub-cordate at base, hispidulous above, glabrous beneath; petioles 1.3-1.8 cm long, 

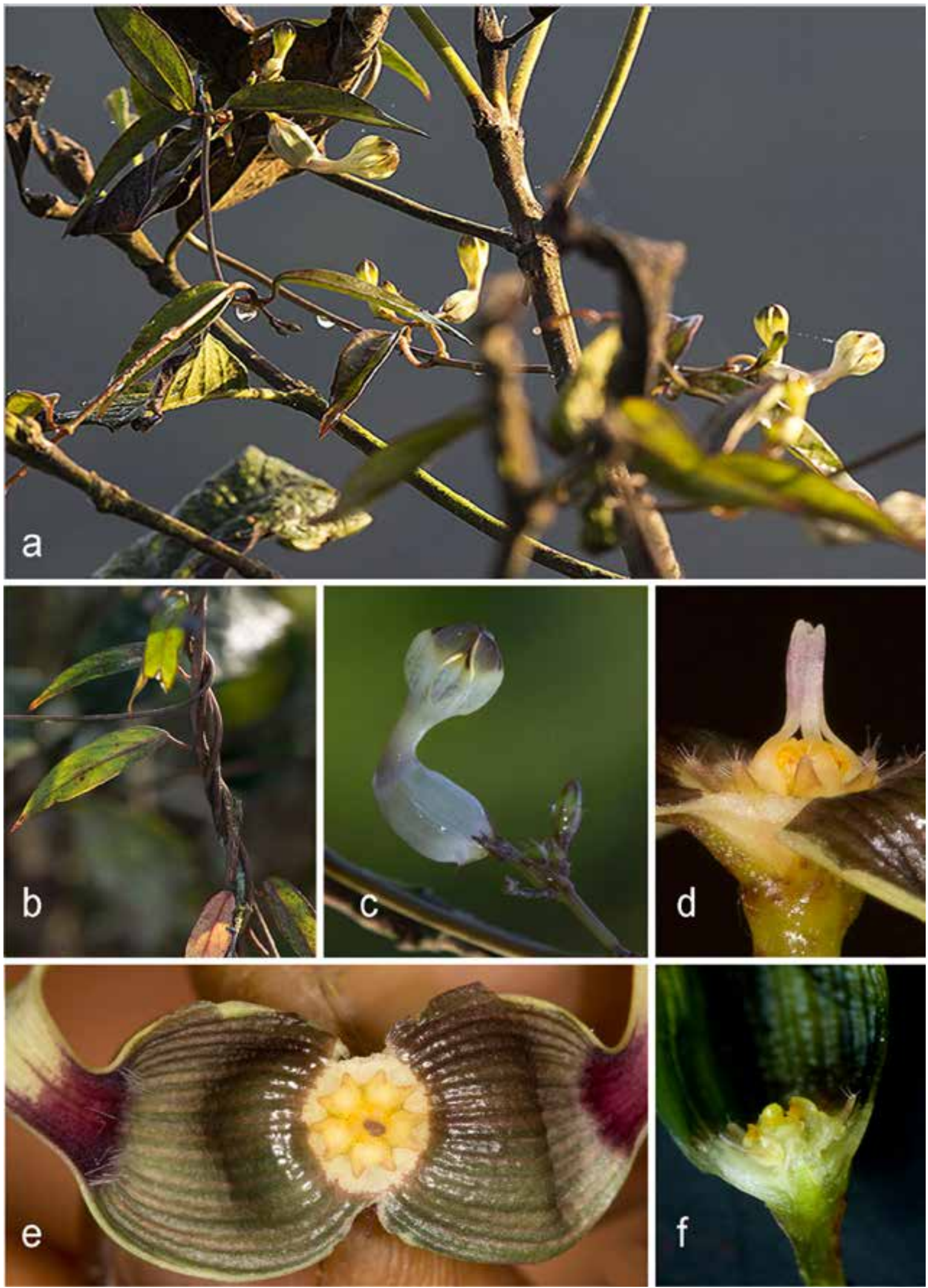

Fig. 30. Ceropegia omissa H. Huber a \& b. Habit; c. Inflorescence; d \& f. Corona-lateral view; e. Corona-top view (Photos Mr. Abhishek Jain). 
channeled above, pubescent. Cymes sub-umbellate, 16-19-flowered, extra-axillary or terminal; peduncles $0.5-4.9 \mathrm{~cm}$ long, slender, pubescent; bracts 3-4.5 mm long, subulate pubescent; pedicels 1-1.6 cm long, pubescent. Sepals 3.5-4 mm long, glabrous, rarely pubescent on the midnerve, linear (subulate). Corolla 3-4 cm long, variable in colour; tube $2.5-3 \mathrm{~cm}$ long, usually straight slightly curved sometimes, gradually dilated towards base, uniformly cylindrical above, narrow at throat, glabrous, greyish-white, dark purple within, with hairs at dilated part, window panes distinct; lobes 5-7 mm long, obovate-acute, connate at tip forming a sub globose-globose cage, glabrous. Corona bi-seriate; outer of 5 shallowly bifid lobes, bowl-shaped, equaling the gynostegium, c. $2 \times 2$ $\mathrm{mm}$, stiff hairy along margins, dark brown to black along margin; inner of 5 erect, white, densely stiff hairy clavate lobes, c. $3 \mathrm{~mm}$ long, purple at tips. Follicles in pairs, c. $11 \mathrm{~cm}$ long, tapering towards apex, glabrous. Seeds $4-6 \times 1.5-2 \mathrm{~mm}$, ovate, oblong, marginate, comose; coma 1-2 cm long, silky white.

Flowering \& fruiting: May-October.

Habitat: Grows along hill slopes in association with Strobilathes callosa and Senecio spp.

Distribution: Endemic to northern Western Ghats of Maharashtra, India.

Specimens examined: INDIA, Maharashtra, Satara district, Mahabaleshwar, 10.07.2003, M.Y. Kamble 2124; s. loc., 07.07.1999, S.R. Yadav s.n.; s.loc., 25.08.1999, S.R. Yadav s.n.; Panchgani (grown in garden), 22.06.2011, S.S. Kambale SUK 2571; Ibid., 16.07.2011, S.S. Kambale SUK 2581; Ibid., 03.07.2014, S.S. Kambale \& B.U. Jadhav SSK 236; Ibid., 03.07.2014, S.S. Kambale SSK 239; Ibid., 21.08.2014, S.S. Kambale SSK 277 (SUK!).

Conservation status: Nayar and Sastry (1987) assessed it as Endangered.

Notes: According to Huber's (1957) classification of the genus, C. panchganiensis belongs Ceropegia sect. Buprestis. While describing the species, Blatter and McCann (1931) compared it with C. lawii Hook.f. This taxon is often misidentified as C. lawii due to morphological similarities. Ansari (1984) treated it as a distinct species and described another closely similar species C. maccannii Ansari. Subsequently
Bruyns et al. (2017) merged it under the latter. However, C. panchganiensis is quite distinct and differs from latter in having yellowish flowers, clavate and hairy inner corona, cupular and hairy outer corona.

Ceropegia pullaiahii Raja Kullayisw., Sandhyar. \& Karupp., Nordic J. Bot. 31(2): 166. 2012. Type: INDIA, Andhra Pradesh, Anantapur district, Nigidi forest, 22.10.2011, K. Raja Kullayiswamy 41845 (holo SKU!; iso MH!; para SKU!). Fig. 32

Vernacular name: Bodanimmatagadda (Telugu).

Perennial twining herbs. Rootstock tuberous; tubers c. 2.5-3.8 cm in diam., depressed globosediscoid; roots fibrous. Stem twining, c. $2.5 \mathrm{~m}$ long, unbranched, glabrous. Lamina $8-16.5 \times$ $0.2-1.2 \mathrm{~cm}$, linear to elliptic-lanceolate, narrower at the both ends, broad at the middle, distinctly hairy above, scabrous beneath, puberulent along margins; petioles $0.6-1.2 \mathrm{~cm}$ long, channeled above, hairy along margins, glabrous otherwise. Cymes 2-3-flowered, extra-axillary; peduncles 3-5 $\mathrm{mm}$ long, terete, puberulous; bracts $2-3 \mathrm{~mm}$ long, linear; pedicels 6.5-6.6 mm long, puberulous. Sepals 0.8-1 cm long, linear, puberulous along midnerve, glabrous otherwise. Corolla $5.5-8.5 \mathrm{~cm}$ long; tube $2.4-3.5 \mathrm{~cm}$ long, erect-slightly curved, abruptly dilated at base, dilated part is globose, cylindrical in middle, funnel-shaped at throat, glabrous within, greenish to yellowish-white blotched with purple, light windows with purple ring at the dilated part, deep purple at middle within, striations up to the base of corolla lobes; lobes linear from deltoid base, 2-4 cm long, reflexed along their back, connate at the tip forming a narrow beak, finely pubescent, dull green at upper $3 / 4^{\text {th }}$ part, deep purple blotched at lower $1 / 4^{\text {th }}$ part. Corona bi-seriate; outer of 5-bifid deltoid lobes, 2.5-3.5 long, c. $7 \mathrm{~mm}$ across, saucer-shaped, glabrous along margins, hairy within, purple along margins, yellow otherwise; inner of linear erect lobes, c. $4.5 \mathrm{~mm}$ long, glabrous, deep purple at the base, yellowish blotched with pink. Pollinarium 1-1.3 mm long. Follicles in pairs, divergent, $8-10.3 \mathrm{~cm}$ long, unequal, blunt at the tip, glabrous, brownish purple. Seeds ovoid, flattened, c. $7 \times 3 \mathrm{~mm}$, dark brown, glabrous, comose; coma c. $2.5 \mathrm{~cm}$ long, silky white.

Flowering \& fruiting: October-December.

Habitat: Grows in grassy slopes of open dry 

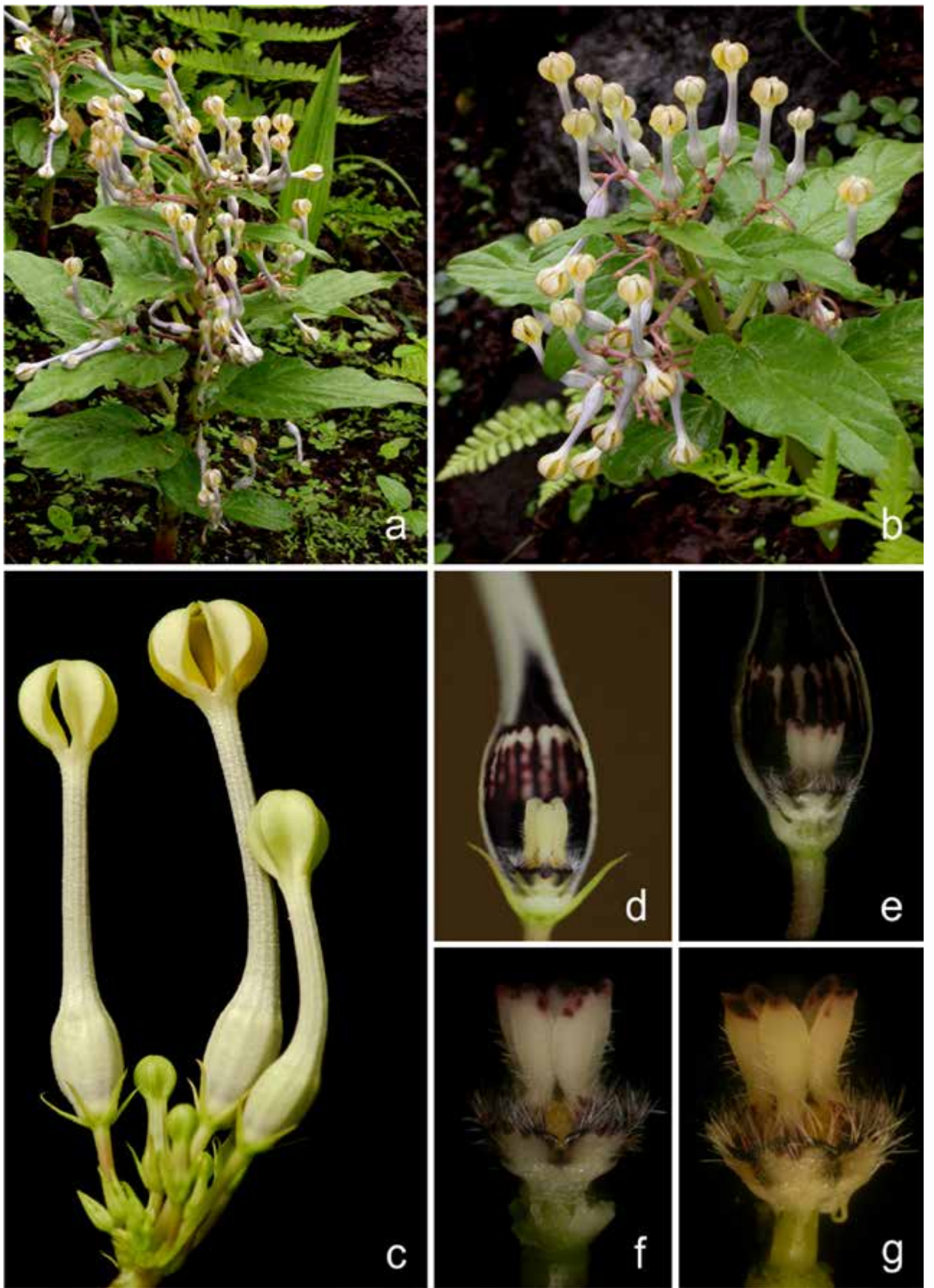

Fig. 31. Ceropegia panchganiensis Blatt. \& McCann: a \& b. Habit; c. Inflorescence; d \& e. Flower L.S.; f \& g. Corona-lateral view. 

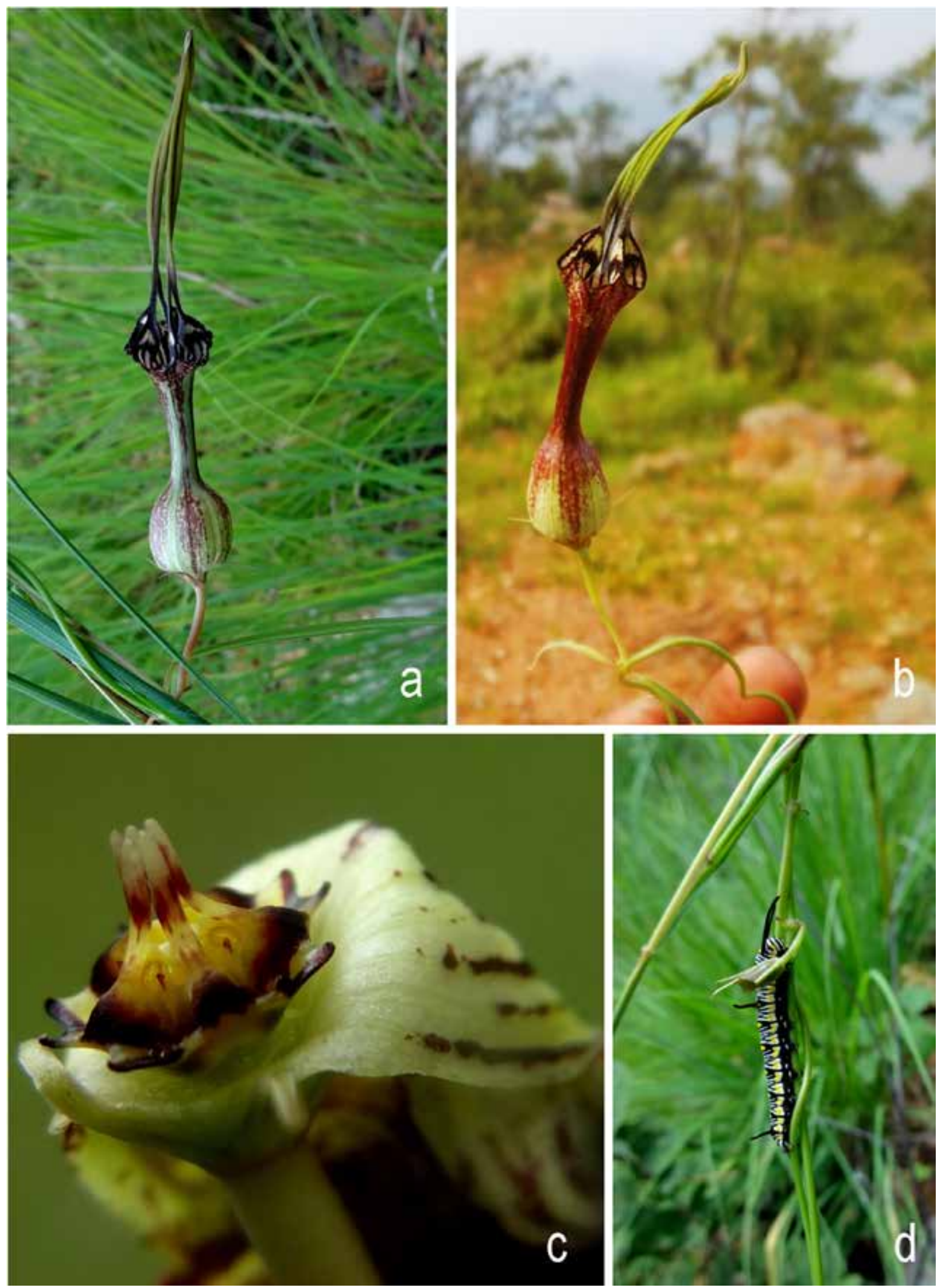

Fig. 32. Ceropegia pullaiahii Raja Kullayisw., Sandhyar. \& Karupp.: a \& b. Flower; c. Corona; d. Larvae of Common Plain Tiger (Danaus chrysippus) on stem. 
deciduous forests at an elevation of $714 \mathrm{~m}$ in association with Cymbopogon coloratus Stapf.

Distribution: So far known only from the type locality in Andhra Pradesh, India.

Specimens examined: INDIA, Andhra Pradesh, Anantapur district, Kadiri forest, Batrepalli, 26.10.2013, S.S. Kambale \& R. Kullaiswamy SSK 98; Ibid., 05.09.2014, S.S. Kambale \& R. Kullayiswamy SSK 302 (SUK!).

Conservation status: During field survey about 20 individuals were located. It is assessed as Critically Endangered [CR: B2 a, b (iii, iv, v)].

Notes: Ceropegia pullaiahii is morphologically similar to C. spiralis Wight, but can be distinguished from it in having linear-slightly twisted corolla lobes and gradually dilated corolla tube. It is also close to $C$. mahabalei Hemadri \& Ansari in floral morphology but differs from it in its glabrous, twining habit and 2-3-flowered cymes.

Ceropegia rollae Hemadri, Bull. Bot. Surv. India 10: 123. 1969; Ansari, Fasc. Fl. India 16: 29. 1984; M.P. Nayar \& Sastry, Red Data Book Indian Pl. 2: 54. 1988; A.P. Jagtap \& N.P. Singh, Fasc. Fl. India 24: 237. 1999; D.K. Mishra \& N.P. Singh, Endem. Threat. Pl. Maharashtra 148. 2001; A.P. Jagtap \& Das Das in N.P. Singh et al., Fl. Maharashtra, Dicot. 2: 355. 2001; M.R. Almeida, Fl. Maharashtra 3A: 237. 2001; Karthik. et al., Fl. Pl. India 1: 163. 2009; Kambale \& S.R. Yadav, Asklepios 115: 37. 2013; Punekar et al., Nelumbo 55: 25. 2013. Type: INDIA, Maharashtra, Pune district, Junnar, Dhak Khilla, 29. 09. 1965, K. Hemadri 107472A (holo CAL!; iso BSI!, $\mathrm{K}$ !, L!).

Ceropegia lawii auct. F. Albers \& Meve, Ill. Handb. Succ. Pl. Asclepiadaceae 85. 2002, p.p., non Hook.f.,1883.

Fig. 33

Vernacular name: Kharpudi (Marathi).

Perennial erect herbs. Rootstock tuberous; tubers 4-5 cm in diam., discoid-subglobose; roots fibrous. Stem 30-200 cm long, terete fleshy, clothed with short coarse bulbous based hairs. Leaves rarely in whorls of three at each node, glabrous; lamina broadly ovate, acute at apex, cordate at base, bulbous based hairs above and along veins beneath, glabrous otherwise; petioles $0.6-1.5 \mathrm{~cm}$ long, channeled above, puberulous. Cymes sub- umbellate, extra-axillary and terminal; peduncles, $1.5-2.7 \mathrm{~cm}$ long, terete, hirsute; bracts $3-5 \mathrm{~mm}$ long, linear-subulate, rarely bi-forked at tip, pubescent along margins; bracteoles c. $2 \mathrm{~mm}$ long; pedicels $0.5-1.5 \mathrm{~cm}$ long, with bulbous based hairs. Sepals 3-5 mm long, linear-lanceolate, puberulous along midnerve. Corolla $2.3-3 \mathrm{~cm}$ long, white; tube 1.3$1.8 \mathrm{~cm}$ long, slightly curved, glabrous, purple and glabrous within, gradually dilated towards base, cylindrical otherwise; lobes white, 1-1.3 × c. 0.35 $\mathrm{cm}$, connate at tips, forming a quadrangular cage, glabrous. Corona bi-seriate; outer saucer-shaped, shorter than gynostegium; lobes slightly divergent at maturity; outer c. $3 \times 3 \mathrm{~mm}$ across, ciliate along margins, yellow; inner of 5 erect linear lobes, c. 3 $\mathrm{mm}$ long, purple spots on either sides at the base, yellow. Follicles in pairs, $5-6 \times 0.4-0.5 \mathrm{~cm}$, linear, terete, tapering at the both ends. Seeds many, 5-6 $\times 2-3 \mathrm{~mm}$, ovate, oblong, marginate; coma 1.5-2 cm long.

Flowering \& fruiting: August-October.

Habitat: Grows along hill slopes amidst Strobilanthes callosa Nees and in lateritic soil on hill tops in association with Chlorophytum glaucum Dalzell, Pinda concanensis (Dalzell) P.K. Mukh. \& Constance and Senecio sp.

Distribution: Endemic to northern Western Ghats of Maharashtra, India.

Specimens examined: INDIA, Maharashtra, Ahmednagar district, Harishchandragad, 06.09.2013, S.S. Kambale SSK 73; Durgawadi, 07.09.2013, S.S. Kambale SSK 77 (SUK!); Pune district, Durgawadi (grown in garden), 15.08.2011, S.S. Kambale SUK 2591; Junnar Durga Khilla, 01.10.1965, K. Hemadri 107547C (CAL!), 107547D (BSI!, CAL!, K!); Junnar, Durgawadi, 30.09.2010, S.S. Kambale SUK 2566; Varandha ghat, 15.07.2003, M.Y. Kamble 2137; Ibid., 11.08.2013, S.S. Kambale SSK 65 (SUK!).

Conservation status: Nayar and Sastry (1987) assessed it as Rare. After thorough survey in Western Ghats it is revealed that species is represented by a few individuals at localities of its occurrence. Habitat modifications, fragmented populations and food value of tuber are the major threats to the species. It is assessed here as Critically Endangered [CR: B2 a, b (iii, iv)]. 
Kambale \& Yadav 63

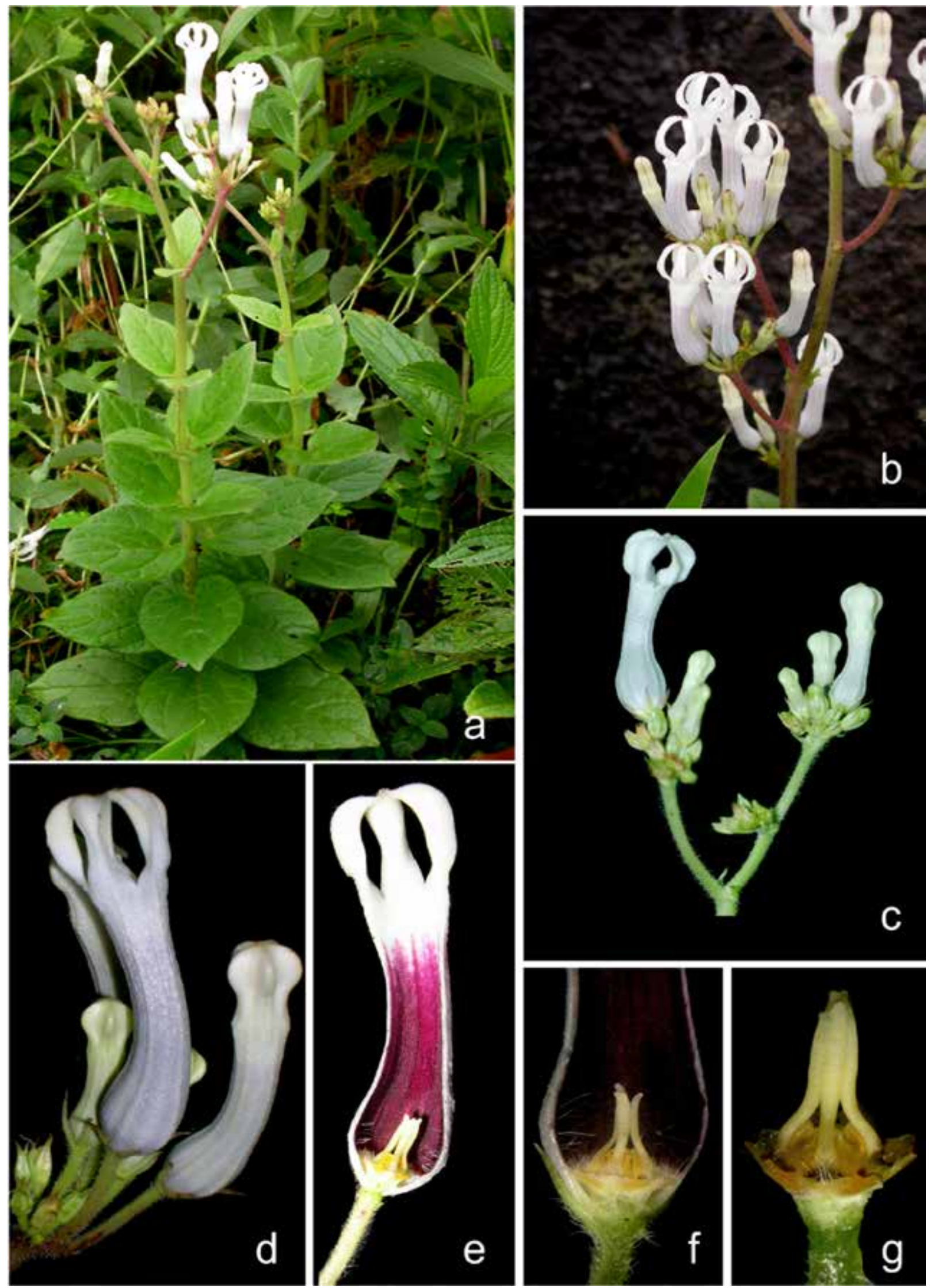

Fig. 33. Ceropegia rollae Hemadri: a. Habit; b-d. Inflorescence; e \& f. L.S of flower; g. Corona-lateral view. 
Notes: Ceropegia rollae is often considered as a synonym of C. lawii Hook.f. (Meve, 2002; The Plant List, 2013). However, field observations revealed that this is rather a distinct species. $C$. rollae usually have terminal inflorescences and saucer-shaped shallow outer corona ( $v s$. extraaxillary inflorescences and deeply bifid bowlshaped corona as in C. lawii). It is also related to C. sahyadrica and C. panchganiensis in its erect habit and white flowers but differs from them in having globose-oblong cage of corolla lobes and cylindrical corolla tube with gradually dilated base.

Ceropegia sahyadrica Ansari \& B.G. Kulk., Indian Forester 97(12): 688. 1971; Ansari, Fasc. Fl. India 16: 29. 1984; M.P. Nayar \& Sastry, Red Data Book Indian Pl. 1: 69. 1987; S.M. Almeida, Fl. Savantwadi 1: 258. 1989; A.P. Jagtap \& N.P. Singh, Fasc. Fl. India 24: 238. 1999; D.K. Mishra \& N.P. Singh, Endem. Threat. Pl. Maharashtra 149. 2001; A.P. Jagtap \& Das Das in N.P. Singh et al., Fl. Maharashtra, Dicot. 2: 357. 2001; Kambale \& S.R. Yadav, Asklepios 115: 38. 2013; Punekar et al., Nelumbo 55: 26. 2013. Type: INDIA, Maharashtra, Sindhudurg district, Amboli Ghat, B.G. Kulkarni 108643A (holo CAL!; iso BSI!, CAL!, K!).

C. lawii auct. T. Cooke, Fl. Bombay 2: 175. 1904; F. Albers \& Meve, Ill. Handb. Succ. Pl. Asclepiadaceae 85. 2002. p.p., non Hook.f. 1883.

C. panchganiensis auct. Reddi, Wildenowia 5(1): 32. 1968; Hemadri, Bull. Bot. Surv. India 10 (2): 125. 1968, non Blatt. \& McCann., 1933.

Perennial erect herbs. Rootstock tuberous; tubers in $2-3.5 \mathrm{~cm}$ in diam., sub-globose; roots fibrous. Stem $20-45 \mathrm{~cm}$ long, generally single from a tuber, occasionally 2-3, usually unbranched, cylindrical, quadrangular at base, pubescent. Lamina variable, $4-8.5 \times 1.2-4.4 \mathrm{~cm}$; lower ones ovate, acute at apex, rounded-cordate at base, upper ones ovatelanceolate, scabrid above, glabrous beneath, ciliolate along nerves beneath; petioles 1-1.5 $\mathrm{cm}$ long, channeled above, pubescent. Cymes umbellate, extra-axillary; peduncles 1-7 cm long, terete, slender, hirsute; bracts $0.4-0.45 \times c .0 .15$ $\mathrm{cm}$, subulate, glabrous; pedicels $0.8-1.7 \mathrm{~cm}$ long, terete pubescent. Sepals $0.5-0.55 \times c .0 .1 \mathrm{~cm}$, linear (subulate), glabrous, rarely hairy, on the midnerve. Corolla 3.8-4.3 cm long, ashy-white; tube $2.5-3.5 \mathrm{~cm}$ long, straight-curved, gradually dilated towards base, dilated part broadly ovate, cylindrical at throat, glabrous, dark purple within lower $3 / 4^{\text {th }}$ portion, white at upper $1 / 4^{\text {th }}$ portion, minutely hairy at the dilated part within; lobes $0.8-1 \mathrm{~cm}$ long, ovate-cordate in shape, connate at tip forming a obconic-obovate cage, $1.5-1.7 \mathrm{~cm}$ across, glabrous, colour of corolla lobe variable, in upper-half it ranges from pale yellow, deep green, olive green, white, at lower half consistently white. Corona bi-seriate; outer of 5 lobes, obtuse, 1-1.5 $\times$ c. $3 \mathrm{~mm}$ across, ciliate along margins and within, yellow; inner of 5 erect linear lobes, c. $5 \mathrm{~mm}$ long, connate at their tip, minutely pubescent at the base, tinged with purple at apex. Follicles in pairs, 5-7 $\mathrm{cm}$ long, terete, tapering at both ends. Seeds many, 4-7 × c. $2 \mathrm{~mm}$, ovate-oblong, marginate, comose; coma 1.5-2 cm long.

\section{Key to the varieties}

1. Corolla lobes olive green, yellow to purple colour in the upper-half; inner corona connivent .................................... var. sahyadrica

1. Corolla lobes bright white; inner corona connivent to divergent var. karulensis

Ceropegia sahyadrica Ansari \& B.G. Kulk. var. sahyadrica

Fig. 34

Vernacular names: Kharpudi, Kartodi (Marathi).

Flowering \& fruiting: August-October.

Chromosome number: $2 n=22$ (Raghavan \& Ansari, 1975).

Habitat: Grows in a variety of habitats like hilltops, high elevation lateritic plateaus and steep slopes.

Distribution: Endemic to northern Western Ghats of Maharashtra.

Specimens examined: INDIA, Maharashtra, Kolhapur district, Kondushi, 24.07.2014, S.S. Kambale \& A.A. Adsul SSK 249; Tillari Ghat, 12.09.2014, S.S. Kambale SSK 305 (SUK); Pune district, Khandala, Duke's point, 10.07.2012, A. Nalawade SSK 4; Torana fort (grown in garden), 06.08.2010, S.S. Kambale SUK 2987; Ibid., 06.08.2011, S.S. Kambale SUK 2590; Ibid., 26.08.2011, S.S. Kambale SUK 2595; 24.07.2014, S.S. Kambale SSK 248 (SUK); Raigad district s.d., H.S. Patil s.n. (SUK!); Raigad fort, 24.07.2014, Sakhare SSK 251(SUK); Ratnagiri district, 
Gothane, 04.07.2011, S.S. Kambale SUK 2573; Ibid., 09.09.2013, S.S. Kambale SSK 61; Ibid., 06.07.2014, S.S. Kambale SSK 238; Gothane Plateau, 19.09.2014, S.S. Kambale SSK 314 (SUK); Sawantwadi district, Danoli, 18.09.1964, R.D. Pataskar 102141 A \& B (BSI!).

Conservation status: Nayar and Sastry (1987) assessed it as Rare. During the present study a good number of populations has been located.

Notes: Ceropegia sahyadrica closely resembles $C$. lawii Hook.f. and C. rollae Hemadri but differs from the former in having a saucer shaped, sparsely hairy outer corona (vs. cupular and densely hairy corona) and broad corolla tube ( $v s$. narrow tube) and differs from the latter in having much dilated corolla tube ( $v s$. gradually dilated tube) and ovate corolla lobes ( $v s$. lanceolate lobes). It is also related to $C$. panchganiensis in its erect habit and white flowers but differs by its dilated corolla tubes, saucer-shaped outer corona and linear erect corona. The variants of this species has been recently described as C. karulensis by Punekar et al. (2013), which is reduced as a variety in the current treatment.

Ceropegia sahyadrica var. karulensis (Punekar, Tamhankar, Lakshmin., Kumaran, Raut, S.K. Srivast. \& Kavade) Kambale \& S.R. Yadav stat. nov. Ceropegia karulensis Punekar, Tamhankar, Lakshmin., Kumaran, Raut, S.K. Srivast. \& Kavade, Nelumbo 55: 17. 2013. Type: INDIA, Maharashtra, Kolhapur district, Gaganbawada, Karul Ghat, 25.08.2005, S.A. Punekar 365 (holo CAL!; iso AHMA!).

Ceropegia lawii auct. Ansari, Fasc. Fl. India 16: 19. 1984; D.K. Mishra \& N.P. Singh, Endem. Threat. Pl. Maharashtra 136. 2001; S.R. Yadav \& Sardesai, Fl. Kolhapur District 285. 2002; Surveswaran et al., Plant Syst. Evol. 281: 53, 60, 2009; Kambale \& Yadav, Asklepios 115: 35. 2013. non Hook.f., 1883.

Fig. 35

Lamina variable; lower ones ovate, rounded or cordate at base, acute at apex, upper ones ovate lanceolate 3-16.3 × 1.1-8.5 cm; petioles $0.5-3.4 \mathrm{~cm}$ long, grooved above, pubescent. Peduncles $c .12 .6$ cm long, hairy; bracts $0.4-0.5 \times c .0 .1 \mathrm{~cm}$, linear; pedicels $0.6-1.4 \mathrm{~cm}$ long, pubescent. Sepals 0.5$0.6 \mathrm{~cm}$, subulate, glabrous. Corolla $1.7-2 \mathrm{~cm}$ long, straight or slightly curved sometimes; tube 1.3-1.5 $\mathrm{cm}$, white, with 10 distinct lines outside, funnelshaped above, minutely hairy at the base of inflated part, dark purple in the lower $2 / 3^{\text {rd }}$ part and white in upper $1 / 3^{\text {rd }}$ part; lobes c. $0.5 \mathrm{~cm}$ long, ovatecordate, forming small globose cage of $0.7 \mathrm{~cm}$ diam. Corona bi-seriate; outer of 5 lobes, saucershaped, shorter than gynostegium, $0.1-0.2 \times c .0 .3$ $\mathrm{cm}$, linear, hairy along the margins, yellow; inner straight, $0.3-0.4 \mathrm{~cm}$, pubescent at apex, yellow.

Flowering \& fruiting: July-September.

Habitat: Grows along steep hill slopes in association with Strobilanthes callosa Nees at an elevation ranging from 400-900 m.

Distribution: Endemic to northern Western Ghats of Maharashtra, India.

Specimens examined: INDIA, Maharashtra, Kolhapur district, Anuskura Ghat, 24.08.2014, S.S. Kambale SSK 288; Bhuibawda Ghat, 24.08.2014, S.S. Kambale \& S.R. Yadav SSK 286 (SUK!); Gaganbawda, Karul Ghat, 27.07.2011, S.S. Kambale SUK 2586 (SUK!); Ibid., 06.08.2011, S.S. Kambale SUK 2589 (SUK!); Ibid., 28.07. 2013, S.S. Kambale SSK 54 (SUK!); Ibid., 06.09.2013, S.S. Kambale SSK 75 (SUK!); Ibid., 15.08.2014, S.S. Kambale SSK 268 (SUK!).

Conservation status: It is reported from 4 localities with 10-15 individuals. It is assessed as Critically Endangered [CR: B2 a, b (iii, iv)].

Notes: While describing this species, Punekar et al. (2013) compared it with C. lawii. They differentiated this taxon based on characters such as caudate-acuminate or abruptly acuminate leaf apices, highly fragrant flowers, usually in terminal sub-umbellate cymes, shorter corolla, glabrous or glabrescent pedicels, white corolla lobes inside and outside and glabrous inner corona lobes. This taxon shares several characters with C. sahyadrica in having white fragrant flowers, saucer-shaped hairy outer corona, hairy corolla tube at dilated part and ovate-cordate corolla lobes. However, it differs from the latter in having relatively smaller flowers, bright white corolla lobes and connivent to divergent inner corona ( $v s$. larger flowers, olive green, yellow to purple corolla lobes and connivent inner corona). After detailed observations on variations of both these taxa, the characters used by Punekar et al. (2013) to describe this species are 

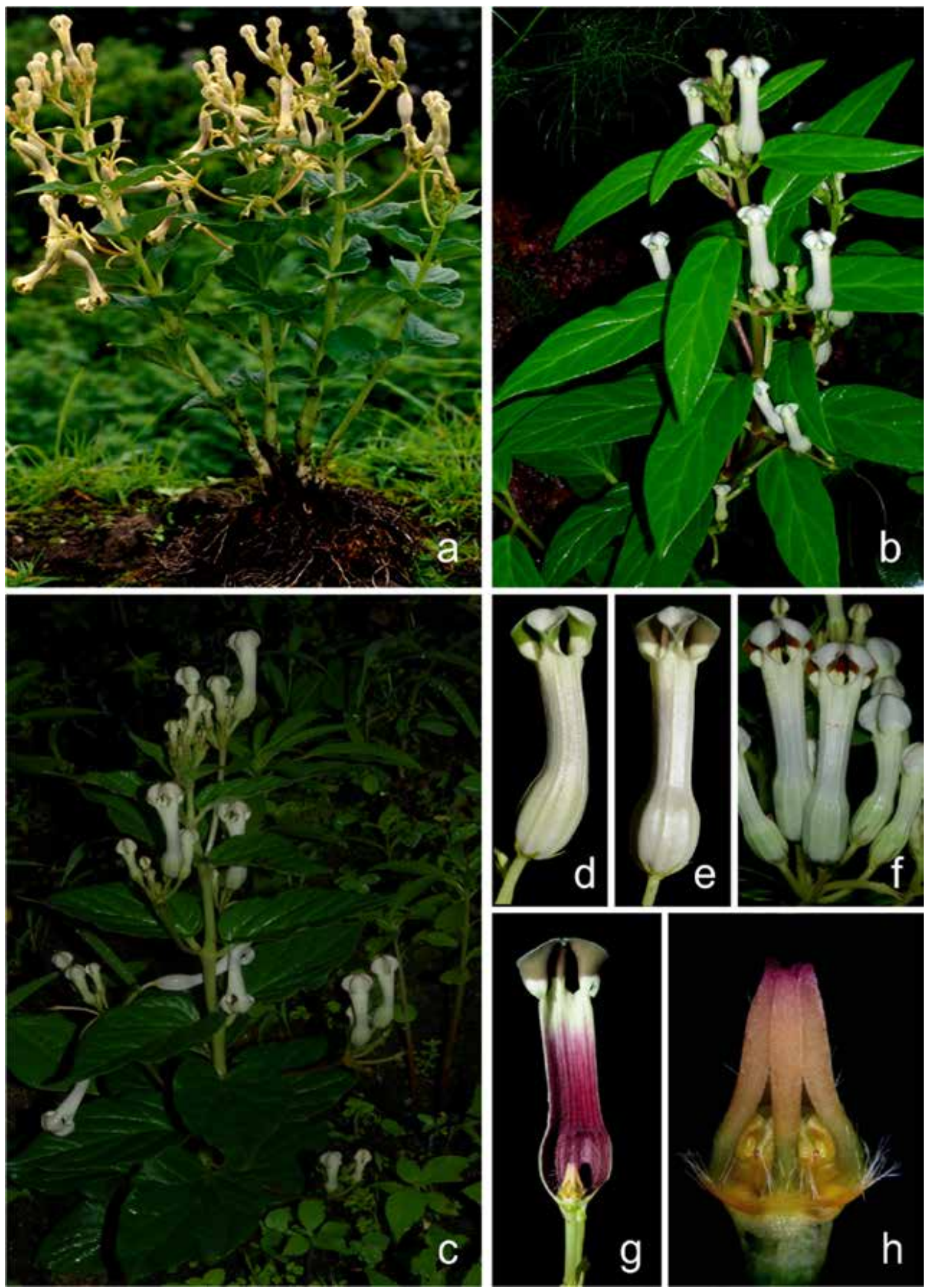

Fig. 34. Ceropegia sahyadrica Ansari \& B.G. Kulk. var. sahyadrica: a-c. Habit; d \& e. Flower; f. Inflorescence; g. Flower L.S.; h. Corona-lateral view. 

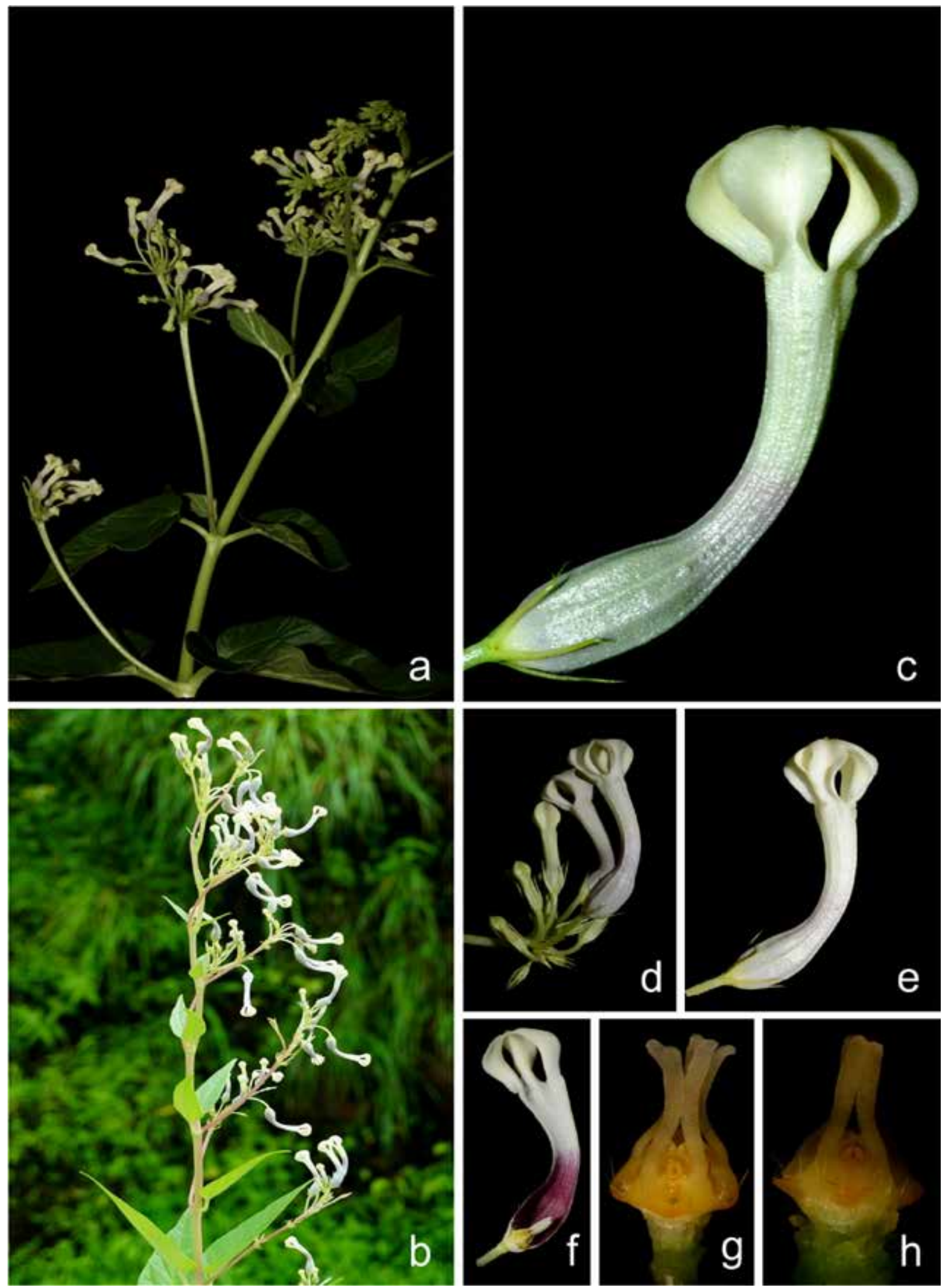

Fig. 35. Ceropegia sahyadrica var. karulensis (Punekar, Tamhankar, Lakshmin., Kumaran, Raut, S.K. Srivast. \& Kavade) Kambale \& S.R. Yadav: a \& b. Habit; c \& e. Flower; $\boldsymbol{d}$. Inflorescence; f. Flower L.S.; g \& h. Corona-lateral view. 
found to be overlapping with C. sahyadrica. Hence authors found no reason to keep it distinct and reduced here as a variety under it. This taxon is also close to C. rollae but differs in having ovate-cordate corolla lobes ( $v s$. linear-lanceolate) and obconicdepressed globose, corolla cages ( $v s$. quadrangular cages).

Ceropegia santapaui Wadhwa \& Ansari, Bull. Bot. Surv. India 10: 95. 1968; Ansari, Fasc. Fl. India 16: 30. 1984; M.P. Nayar \& Sastry, Red Data Book Indian Pl. 2: 56. 1988; S.D. Deshp. et al., Fl. Mahabaleshwar, Maharashtra 1: 362. 1993; A.P. Jagtap \& N.P. Singh, Fasc. Fl. India 24: 238. 1999; D.K. Mishra \& N.P. Singh, Endem. Threat. Pl. Maharashtra 151. 2001; A.P. Jagtap \& Das Das in N.P. Singh et al., Fl. Maharashtra, Dicot. 2: 357. 2001; M.R. Almeida, Fl. Maharashtra 3A: 238. 2001; F. Albers \& Meve, Ill. Handb. Succ. Pl. Asclepiadaceae 98. 2002; Kambale \& S.R. Yadav, Asklepios 115: 38. 2013. Type: INDIA, Maharashtra, Satara district, way from Mahabaleshwar to Mahad, 19.08.1966, B.M. Wadhwa 109640A (holo CAL!; iso CAL!, K!, BSI!).

Fig. 36

Vernacular name: Kharpudi (Marathi).

Perennial twining herbs. Rootstock tuberous; tubers $1-3 \mathrm{~cm}$ in diam., sub-globose; roots fibrous. Stem c. $2 \mathrm{~m}$ long, usually one from tuber, rarely two, slender, usually unbranched, rarely branched, glabrous, pubescent at younger parts. Lamina variable, lower ones ovate-acuminate, $8.4-12.3 \times$ $3.2-4.3 \mathrm{~cm}$, upper ones linear-lanceolate, 6.2-9.4 $\times 0.9-1.6 \mathrm{~cm}$, bulbous based hairs above, ciliolate along nerves beneath, glabrous otherwise; petioles 0.3-4.5 cm long, channeled above, with 2 black glands on either sides at the base, glabrous. Cymes sub-umbellate, 6-10-flowered, extra-axillary; peduncles $0.6-9 \mathrm{~cm}$ long, terete, puberulous; bracts $2-5 \times$ c. $1.5 \mathrm{~mm}$, subulate, glabrous; pedicels $0.8-$ $2.5 \mathrm{~cm}$, pubescent-puberulous. Sepals 3-3.5 mm long, subulate, hairy on midnerve. Corolla 1-1.3 cm long, white or greenish-yellowish-white; tube c. $1 \mathrm{~cm}$ long, angular, straight or slightly curved, glabrous, purple and glabrous within, gradually dilated towards base; lobes c. $6 \mathrm{~mm}$ long, connate at tips, forming a sub-globose cage, glabrous, yellowish-white. Corona uni-seriate of 5 elongated lobes, conical, pale yellow with purple blotching at lower half, white in upper-half; inner c. $3 \mathrm{~mm}$ long, convergent, connate at tip, subulate, densely stiff hairy on the dorsal side. Follicles in pairs, 5.5-7.2 $\mathrm{cm}$ long, tapering at both ends, glabrous. Seeds many, c. $7 \times 3 \mathrm{~mm}$, ovate, oblong, prominently margined; coma c. $1.5 \mathrm{~cm}$ long.

Flowering \& fruiting: August-October.

Chromosome number: $2 n=22$ (Raghavan \& Ansari, 1975).

Habitat: Grows along hill slopes in soil deposited on rocks in association with Tripogon lisboae Stapf.

Distribution: Endemic to northern Western Ghats of Maharashtra, India (Mishra \& Singh, 2001).

Specimens examined: INDIA, Maharashtra, Ratnagiri district, Gothane, 04.07.2011, S.S. Kambale SUK 2574 (SUK); Ibid., 19.09.2014, S.S. Kambale SSK 313 (SUK!); Gothane plateau, 05.08.2003, M.Y.Kamble2163 (SUK); Ibid., 08.2003, S.P. Gaikwad 427(SUK); Pophali Ghat, 10.08.2002, S.P. Gaikwad s.n. (SUK); Satara district, Koyanagar, 23.08.1999, S.A. Mohite s.n.; Kumbharli Ghat, 22.07.2003, M.Y. Kamble s.n.; Ibid., 19.08.2011, S.S. Kambale SUK 2593; Ibid., 21.08.2014, S.S. Kambale SSK 279 (SUK); way from Mahabaleshwar to Mahad, 13.09.1966, B.M. Wadhwa \& M.Y. Ansari 109651A (CAL!, BSI!).

Conservation status: Nayar and Sastry (1987) assessed it as Rare. After type collection this species has been collected from similar habitats in Western Ghats. However, it is represented by few individuals at each locality and has fragmented distribution. In present study it has assessed as Data Deficient (DD).

Notes: Ceropegia santapaui is closely similar to $C$. huberi but can be distinguished from the latter by its corolla tube longer than lobes and sub-globose cage of corolla lobes $(v s$. shorter corolla tube than corolla lobes and depressed discoid cage as in $C$. huberi).

Ceropegia vincifolia Hook., Bot. Mag. 66: t. 3740. 1839 emend. Ansari, Bull. Bot. Surv. India 13(3 \& 4): 187. 1971; Dalzell \& A. Gibson, Bombay Fl. 153. 1861; M.P. Nayar \& Sastry, Red Data Book Indian Pl. 2: 59. 1988; Shetty \& Singh., Fl. Rajasthan 2: 479. 1991; S.D. Deshp. et al., Fl. Mahabaleshwar, Maharashtra 1: 362. 1993; A.P. Jagtap \& N.P. Singh, Fasc. Fl. India 24: 240. 1999; D.K. Mishra \& N.P. Singh, Endem. Threat. Pl. Maharashtra 152. 2001; A.P. Jagtap \& Das Das in N.P. Singh et al., Fl. Maharashtra, Dicot. 

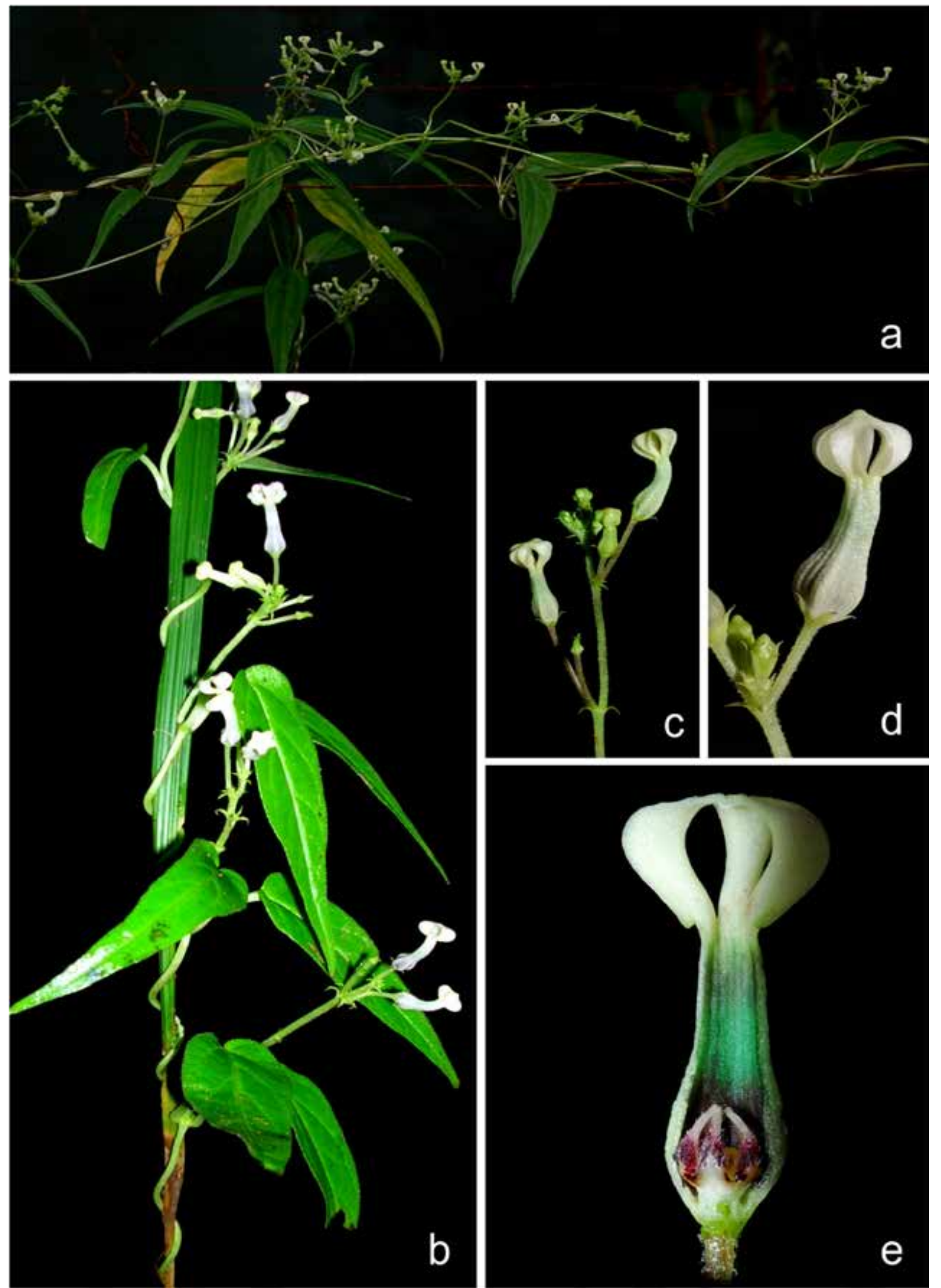

Fig. 36. Ceropegia santapauiWadhwa \& Ansari: a \& b. Habit; c. Inflorescence; d. Flower; e. Flower L.S. 
2: 357. 2001; M.R. Almeida, Fl. Maharashtra 3A: 239. 2001; F. Albers \& Meve, Ill. Handb. Succ. Pl. Asclepiadaceae 106. 2002; Karthik. et al., Fl. Pl. India 1: 164. 2009; Kambale \& S.R. Yadav, Asklepios 115: 38. 2013. Ceropegia hirsuta Wight \& Arn. var. vincifolia (Hook.) Hook.f., Fl. Brit. India 4: 72. 1883, as 'var. vincaefolia'. Lectotype (designated by Ansari, 1984): Bot. Mag. t. 3740. 1839.

Ceropegia stocksii Hook.f., Fl. Brit. India 4: 74. 1883; T. Cooke, Fl. Bombay 2: 243. 1905. Type: not located or apparently none preserved.

Ceropegia polyantha Blatt. \& McCann, J. Bombay Nat. Hist. Soc. 34. 936. 1931. Lectotype: INDIA, Maharashtra, Mahabaleshwar, Lingmala hills, s.d., McCann \& Fernadez 3470A (BLAT).

Ceropegia oculata var. subhirsuta H. Huber, Mem. Soc. Brot. 12: 65. 1957. Type: INDIA, Maharashtra, Mumbra, s.d., Shenoy 4057 (BLAT).

Fig. 37

Vernacular names: Kharpudi, Khapar-khutti, Khaper-Halda (Marathi).

Perennial twining herbs. Rootstock tuberous; tubers sub-spherical, $4-5 \mathrm{~cm}$ in diam.; roots fibrous. Stem c. $3 \mathrm{~m}$ long, terete, slightly swollen at nodes, usually branched, glabrous, greenish-dark purple. Lamina 5.3-16.0 × 2.7-7.3 cm, leaf-size gradually decreasing above, ovate-lanceolate, acuteacuminate at apex, cordate at base, membranous, sparsely hairy above, along margins and nerves beneath; petioles 1.1-4 cm long, channeled above, glabrous. Cymes umbellate, 9-17-flowered, extraaxillary; peduncles $1.3-5.5 \mathrm{~cm}$ long, terete, hirsute; bracts $c .0 .35 \times 0.1 \mathrm{~mm}$, subulate, glabrous (rarely hairy at the tip); pedicels $0.5-1.3 \mathrm{~cm}$ long, terete, glabrous. Sepals $0.4-1.1 \mathrm{~cm}$ long, ciliolate along midnerve, subulate, glabrous otherwise. Corolla $3.5-8.0 \mathrm{~cm}$ long; tube $2.1-4.0 \mathrm{~cm}$ long, straight, abruptly dilated at base, funnel-shaped at throat, glabrous with ring of downwardly directed hairs at the mouth of dilated part within, greyishgreen-pale green, mottled with deep purple spots above the middle and at the dilated part, deep purple at narrower part within, pale green above at the mouth, horizontal light windows present at the mouth of dilated part; lobes $1.3-3 \mathrm{~cm}$ long, connate at tip forming an ovoid-oblong or ovoid head, almost equal to or shorter than tube, linearoblong above from ovate-deltoid base, rarely twisted, pubescent within and hairy along margins, colour variable, greenish-brown, reddish-brown, purple to dark green at upper-half, usually pale yellowish-green with faint purple lines in the lower half. Corona bi-seriate, stipitate, 5-6 mm long; outer of 5 emarginate or shortly bifid lobes, c. $4 \times 4 \mathrm{~mm}$, slightly larger than gynostegium, ciliate along margins and within, yellow with purple margin; inner of 5 erect to erecto-divergent lobes, $4 \mathrm{~mm}$ long, ligulate, linear-spathulate, oblanceolate, rhomboid, alternating with outer corona. Pollinarium c. $0.2 \times 0.25 \mathrm{~mm}$. Follicles in pairs, 11.5-15 long, unequal, cylindrical, tapering towards apex, glabrous, greenish-purple. Seeds c. $7 \times 4 \mathrm{~mm}$ ovate-oblong, flattened with broad margins, comose; coma $c .3 \mathrm{~cm}$ long.

Flowering \& fruiting: August-September.

Habitat: Grows around bushes in lateritic soil and along hill slopes in association with Strobilanthes callosa Nees and Euphorbia antiquorum L.

Distribution: India (Kerala, Maharashtra, Rajasthan).

Specimens examined: INDIA, Goa, Porvorim slopes, 07.09.2007, A. Prabhugaonkar 537 (Goa University Herbarium!). Kerala, Palakkad district, Silent Valley National Park, 30.08.2012, A.R. Kulavmode E S.S. Kambale SUK 3132 (SUK!). Maharashtra, Kolhapur district, Gargoti, Kondushi, 03.10.1998, P.D. Mahekar \& V.B. Shimpale 5819 (SUK!); Mumbai suburban, SGNP Borivali, 30.08.2012, S.S. Kambale SSK 16; Ibid., 07.09.2013, S.S. Kambale SSK 83; SGNP (grown in garden), 23.10.2013, S.S. Kambale SSK 94; 15.08.2014, S.S. Kambale SSK 275 (SUK!); Nandurbardistrict, Toranmal, Kalapani, 16.08.1965, R.D. Pataskar 105809 (BSI!); Pune district, Katraj, 08.1956, G.S. Puri BSI 2965; Mahar-Dara, 10.09.1964, M.Y. Ansari 101583B; Ibid., 09.08.1956, G.S. Puri 5689; Sinhgadh forest, 31.08.1907, R.K. Bhide s.n.; (BSI!); Raigad district, Karjat, Thakarwadi hills, Mhatarbanala, 18.09.1962, R.S. Rao 81582 (BSI!); Satara district; Kas, 09.1991, M.P. BachulkarCholekar 5165 (SUK!); Ibid., 01.09.2003, M.Y. Kamble 2133; Ibid. (grown in garden), 05.10.2011, S.S. Kambale SUK 3133; 30.08.2012, S.S. Kambale SSK 15; Ibid., 12.08.2013, S.S. Kambale SSK 69; Ibid., 19.09.2014, S.S. Kambale SSK 318; Mahabaleshwar, Lingmala, 27.09.1930, C. McCann 3442 (CAL!); Patan tehsil, Valmiki, 12.10.2013, M.K. Mane \& S.S. Kambale SSK 101 (SUK!); way from Satara to Mahabaleshwar, Machutar forest, 10.10.1960; 


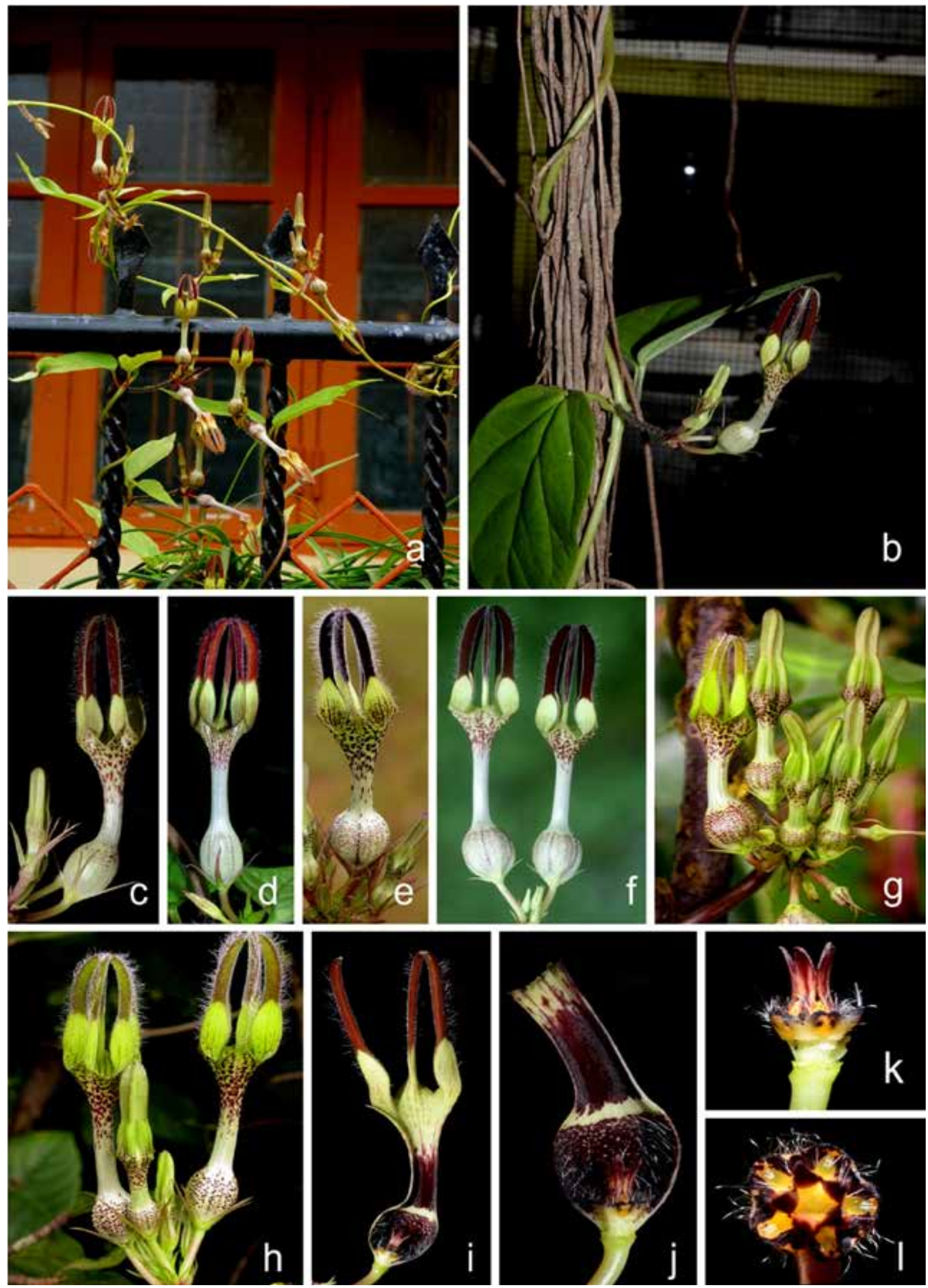

Fig. 37. Ceropegia vincifolia Hook.: a \& b. Habit; c-h. Floral variations; i \& j. Flower L.S.; k. Corona-lateral view; I. Corona-top view. 
M.Y. Ansari 67507(SUK!); Thane district, Dahisar Range, Takmak fort hills, 16.09.1968, K.V. Bhillare 116748 (BSI!).

Conservation status: Nayar and Sastry (1987) assessed it as Rare while Mishra and Singh (2001) as Endangered. In present study few fragmented populations have been observed.

Notes: Ceropegia vincifolia is close to C. oculata but differs in having corolla lobes as long as tube (vs. shorter than tube), horizontal light windows ( $v s$. vertical light windows) and densely hairy within dilated part ( $v s$. glabrous) and emarginate outer corona lobes ( $v s$. bifid corona lobes). It is variable in colour, blotching pattern and length of corolla lobes. Colour in upper-half of corolla lobes varies from pale green, deep green to reddish. Shape of cage varies from conical to obovoid and the shape of dilated portion of tube varies from globular to ovoid. The corolla lobes are sometimes slightly spirally-coiled. Hooker (1883) and Cooke (1905) reduced $C$. vincifolia to a variety of $C$. hirsuta but, Huber (1957) treated them as conspecific. Subsequently, Ansari (1971) made critical observations on all the populations and concluded that C. vincifolia should be considered as distinct species and emended the description.

\section{Ceropegia sect. Janthina}

Ceropegia sect. Janthina H. Huber, Mem. Soc. Brot. 12: 29. 1957; Bruyns, S. African J. Bot. 112: 429. 2017. Type: Ceropegia elegans Wall.

Evergreen climbers with fibrous or slightly swollen roots. Leaves cordate to ovate-lanceolate, petiolate, glabrous to puberulous. Flowers $2-8$ in cymes. Corolla tube usually with ring of hairs at the throat of dilated part within.

Distribution: Eleven species in the world; southeast Asia (India, Sri Lanka, Malaysia), Malesia (Philipines), northern Australia. In India the section is represented by 10 species and is distributed in the southern Western Ghats up to Andaman Islands (Table 1).

\section{Key to the species of sect. Janthina H. Huber}

1. Corolla lobes equal or longer than tube .......... 2

1. Corolla lobes shorter than tube ......................... 4

2. Inner corona lobes clavate C. andamanica
2. Inner corona lobes linear 3

3. Dilated part of corolla tube globose C. decaisneana

3. Dilated part of corolla tube elongated C. metziana

4. Corolla c. $2 \mathrm{~cm}$ long C. maculata

4. Corolla longer than $2 \mathrm{~cm}$ 5

5. Outer corona shorter than inner 6

5. Outer corona equal to the inner C. elegans

6. Corolla lobes hairy along margins 7

6. Corolla lobes glabrous along margins 9

7. Inner corona lobes clavate C. intermedia

7. Inner corona lobes linear 8

8. Cymes up to 3-flowered C. manoharii

8. Cymes up to 7-flowered .C. schumanniana

9. Cymes up to 6-flowered C. thwaitesii

9. Cymes up to 3-flowered C. gardneri

Ceropegia andamanica Sreek., Veenak. \& Prashanth, Blumea 43(1): 215. 1998; Karthik. et al., Fl. Pl. India 1: 160. 2009. Type: INDIA, Andaman Islands, South Andaman, Mt. Harriet National Park, 14.12.1995, Sreekumar \& Veenakumari 15493 (holo CAL!; iso PBL, L).

Fig. 38

Perennial twining herbs with watery latex. Rootstock with fleshy roots. Lamina 5-15 × 1-5 $\mathrm{cm}$, lanceolate to elliptic-lanceolate, chartaceous, rounded or shallowly cordate at base, acuteacuminate at apex, glaucous beneath, glabrous, deep-green above; petioles $0.5-2 \mathrm{~cm}$ long, slender. Cymes 3-8-flowered, extra-axillary. Flowers purplish-mottled; peduncles 1-2.5 cm long, glabrous; bracts 3-4 mm long, subulate, glabrous; pedicels $0.5-2 \mathrm{~cm}$ long, terete, glabrous. Sepals $0.5-1 \mathrm{~cm}$ long, subulate, glabrous. Corolla 5-12 cm long; tube 1.5-2 cm long, dilated at base, subcylindrical, funnel-shaped at throat; lobes 3-7 cm long, connate at tip, twisted, whip like, hairy, purplish. Corona bi-seriate; outer of 5 ovate-retuse bifid lobes, c. $3 \times 2 \mathrm{~mm}$, ciliate along margins and within; inner of 5 erect club shaped lobes, 1.8-2 $\times$ c. $1 \mathrm{~mm}$, glabrous. Fruits and seeds not seen.

Flowering: November-December. 
Habitat: Occurs along road sides at forest edges and rocky loam in primary evergreen forests. It grows in association with Mallotus resinosus (Blanco) Merr. and Phaulopsis imbricata Sweet.

Distribution: Endemic to Andaman Islands, India.

Specimens examined: INDIA, Andaman Islands, Near Herbertabad, 29.11.1975, N.G. Nair 3169 (PBL!); South Andaman, Mount Harriet, 07.12.1989, S.P.K. Mathew 20416 (PBL!); Ibid., 11.12.1997, N. Balachandran 0804 (FRLH!).

Conservation status: Ceropegia andamanica is restricted to the type locality. After type collection it has been collected only once by Mr. N. Balachandran from the same locality. Area of occupancy is less than 50 sq. $\mathrm{km}$. Therefore, it is assessed here as Critically Endangered [CR: B2 a, b (iii, v)].

Notes: Ceropegia andamanica is similar to $C$. metziana Miq. but differs by its glabrous leaves and peduncles, longer corolla (c. $12 \mathrm{~cm}$ long), club shaped inner corona and absence of ring of hairs at the throat of dilated portion of corolla tube.

Ceropegia decaisneana Wight, Ic. Pl. Ind. Or. 4: t. 1259. 1848; Hook.f., Fl. Brit. Ind. 4: 73. 1883; Trimen, Handb. Ceylon Pl. 3:166. 1895; Gamble, Fl. Madras 4: 859. 1921; H. Huber, Mem. Soc. Brot. 12: 62. 1957; B.D. Sharma et al., Fl. Karnataka, Analysis 166. 1984; Ansari, Fasc. Fl. India. 16: 13. 1984; S.R. Sriniv. in Henry et al., Fl. Tamil Nadu Ind., Ser I, Analysis 2: 83. 1987; M.P. Nayar \& Sastry, Red Data Book Indian Pl. 2: 41. 1988; Bruyns, Rheedea 7(2): 109. 1997; A.P. Jagtap \& N.P. Singh, Fasc. Fl. India 24: 220. 1999; F. Albers \& Meve, Ill. Handb. Succ. Pl.

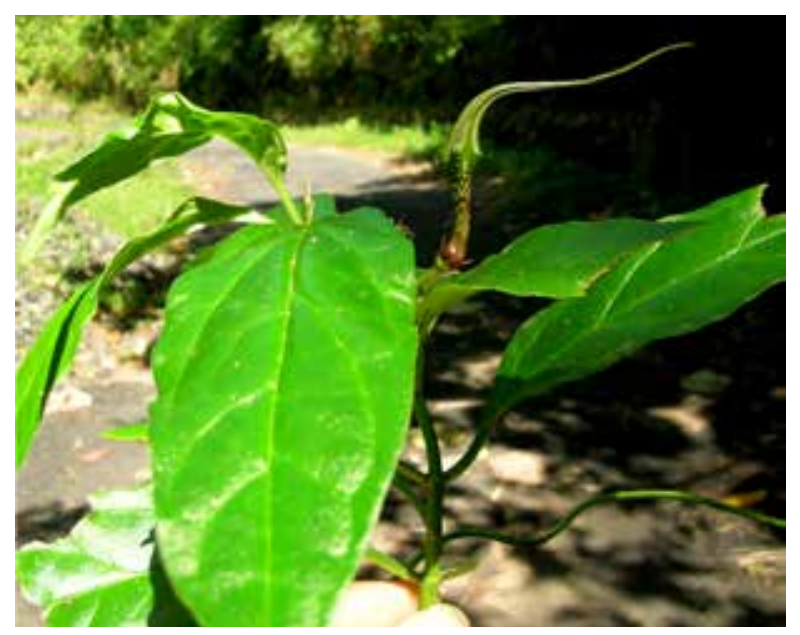

Fig. 38. Ceropegia andamanica Sreek., Veenak. \& Prashanth: a. Habit.
Asclepiadaceae 74. 2002; T.S. Nayar et al., Fl. Pl. Kerala 85. 2006; Karthik. et al., Fl. Pl. India 1: 161. 2009; Kambale \& S.R. Yadav, Asklepios 115: 31. 2013. Lectotype (Step I designated by Huber, 1957; Step II designated by Kambale \& Yadav, 2015): INDIA, Tamil Nadu, Sisparah ghats (Nilgiris), s.d., R. Wight s.n. (BM001051239 image!).

C. brevicollis Hook.f., Fl. Brit. India 4: 74. 1883; Gamble, Fl. Madras 2: 604. 1957; B.D. Sharma et al., Fl. Karnataka, Analysis 165. 1984. C. decaisneana var. brevicollis (Hook.f.) H. Huber, Mem. Soc. Broter. 12: 62. 1957. Lectotype (designated by Kambale \& Yadav, 2015): INDIA, Kerala, Thiruvananthapuram district, Annamalay hills, s.d., s.coll. s.n. (K000857806 image!).

Fig. 39

Perennial twining herbs. Rootstock with fascicledfleshy roots. Stem 3-4.5 m long, branched, quite woody at base, soft upwards, $3 \mathrm{~mm}$ in diam., glabrous. Lamina 4.6-20.5 × 1.7-6.0 cm, ovatelanceolate to elliptic-oblong, acuminate at apex, rounded at base, pubescent above, along margins and beneath, deep green above, pale below; petioles 1.2-4.5 cm long, channeled above, sparsely pilose. Cymes umbellate, 4-8-flowered, extra-axillary; peduncles usually longer than pedicels, 2.4-10.5 cm long, terete, both hairy, hairy in one row; pedicels $1-7 \mathrm{~cm}$ long, glabroushairy. Corolla $4.5-7.5 \mathrm{~cm}$ long, outside minutely hairy in buds; tube $3.3-4.3 \mathrm{~cm}$ long, dilated at base, cylindrical at middle, funnel-shaped at throat, c. $2 \mathrm{~cm}$ across at throat, glabrous except for ring of hairs at the throat of dilated part, greenish-yellow, blotched with purple at middle and throat outside, deep purple within, greyishwhite vertical light windows present at dilated part within; lobes $2-4 \mathrm{~cm}$ long, deltoid in lower half, spathulate above, completely reflexed along margins. Corona bi-seriate; outer of 5-bifid lobes, hairy along margins and within; inner erect, connivent, linear-spathulate, glabrous. Follicles $c$. $23 \mathrm{~cm}$ long, blunt at tips.

Flowering \& fruiting: September-January.

Habitat: Grows along edges of evergreen forests.

Distribution: South India (Karnataka, Kerala, Tamil Nadu) and Sri Lanka.

Specimensexamined:INDIA, Kerala, Kollam district, Pandimotta, 11.01.1994, E.S. Santhosh 19180 

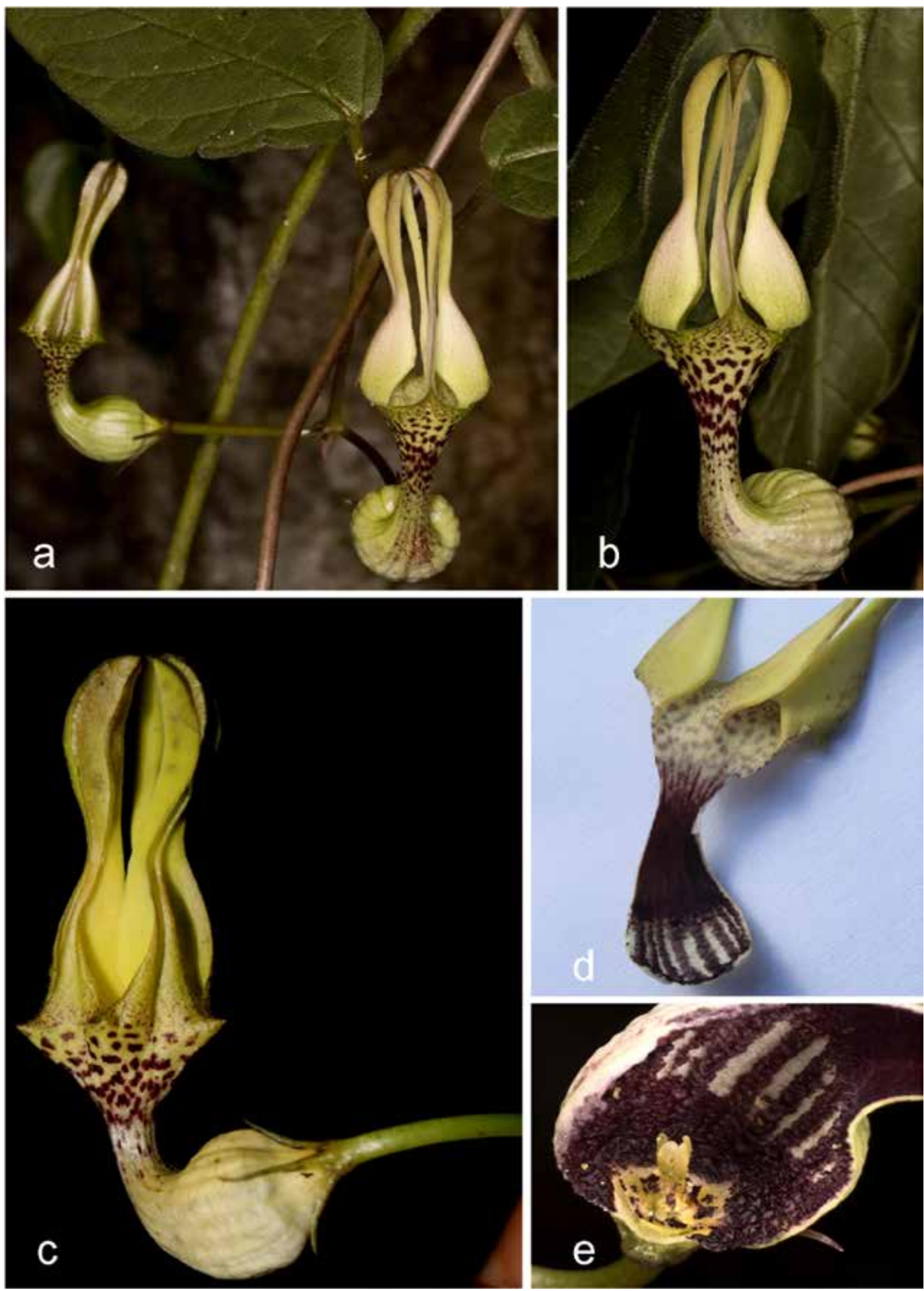

Fig. 39. Ceropegia decaisneana Wight: a. Inflorescence; b \& c. Flower; d. Flower L.S.; e. Corona. (Photos A.R. Kulavmode \& Navendu Page). 
(TBGT!); Palakkad district, Silent Valley National Park, s.d., N.C. Nair 64328 (MH); Sispara Ghat, 1921, J.S. Gamble s.n. (MH!); Thiruvananthapuram district, Othaikkal, 07.11.1904, C.A. Barber 6194; Ponmudi, Mr. Valentine's estate, 02.12.1905, C.A. Barber 7336(MH!). Tamil Nadu, Kariabolamallai, 1925, C.E.C. Fischer 3654 (CAL!).

Conservation status: It is assessed as Data Deficient (DD).

Notes: Ceropegia decaisneana is close to C. metziana in having longer corolla lobes but, can be distinguished by its bifid outer corona.

Ceropegia elegans Wall., Bot. Mag. 57: t. 3015. 1830; Hook.f., Fl. Brit. India 4: 68. 1883; Gamble, Fl. Madras 4: 857. 1921; K.M. Matthew, Fl. Tamil Nadu Carnatic 2: 936. 1983; B.D. Sharma et al., Fl. Karnataka, Analysis 166. 1984; Ansari, Fasc. Fl. India 16: 14. 1984; S.R. Sriniv. in Henry et al., Fl. Tamil Nadu Ind. Ser I, Analysis 2: 83. 1987; A.P. Jagtap \& N.P. Singh, Fasc. Fl. India 24: 220. 1999; K.M. Matthew, Fl. Palni Hills, S. India 2: 802. 1999; T.S. Nayar et al., Fl. Pl. Kerala 85. 2006; Karthik. et al., Fl. Pl. India 1: 161. 2009; Kambale \& S.R. Yadav, Asklepios 115: 31. 2013. Ceropegia elegans var. elegans auct. H. Huber, Mem. Soc. Brot. 12: 63. 1957; Bruyns in Rheedea 7(2): 111. 1997, non Wallich, 1830. Lectotype (designated by Ansari, 1984): Bot. Mag. t. 3015. 1830.

C. walkerae Wight, Icon. Pl. Ind. Orient. 4: 15. t. 1266. 1850. C. elegans var. walkerae (Wight) Trimen, Handb. Fl. Ceylon 3: 165.1895. Lectotype (designated by Singh, 2015): Wight, Icon. Pl. Ind. Orient. t. 1266. 1848.

C. similis N.E. Br., Gard. Chron. 40: 383. 1906. Type: not located or apparently none preserved.

C. ledgeri N.E. Br., Bull. Misc. Inform. Kew 3: 121. 1913. Type: not located or apparently none preserved.

C. sphenanantha Wight \& Arn. in Wight, Contr. Bot. India 31. 1834; Decne. in DC., Prodr. 8: 643. 1844. Lectotype (designated by Singh, 2015): INDIA, Tamil Nadu, Neelgherry (Nilgiri), s.d., N. Wallich Asclep. no. 6 (E00179556 image!).

C. mysorensis Wight, Icon. Pl. Ind. Orient. t. 846. 1843-1845. Lectotype (designated by Singh, 2015): INDIA, Karnataka, Mysore, 12.1834, R. Wight 2203 (K000894256 image!; isolectotype E00179762 image!, K000894255 image!, NY00318546 image!).

Fig. 40

Perennial extensive twining herbs. Rootstock fibrous-fascicled. Stem reaches a considerable height, profusely branched, glabrous. Lamina 5.3-7.4 × 4-6 cm, ovate-oblong, acute-acuminate at apex, rounded-sub-cordate at base with few glands, glabrous with hairy along margins and on prominent nerves beneath, dark green above, pale below; petioles $2.2-3.5 \mathrm{~cm}$ long, channeled above, puberulous. Cymes sub-umbellate, 2-5-flowered, extra-axillary or lateral; peduncles $1-2.4 \mathrm{~cm}$, terete, dotted, glabrous; bracts $4-4.5 \mathrm{~mm}$ long, linear (subulate), dotted, glabrous; pedicels 1.3-1.5 cm long, slender, dotted, glabrous. Sepals 5, 5-6 $\mathrm{mm}$ long, acute-lanceolate, tinged with purple along mid nerve. Corolla $c .4 \mathrm{~cm}$ long; tube c. 3.2 $\mathrm{cm}$ long, abruptly dilated at base, cylindrical at middle, funnel-shaped at throat, glabrous, ring of downwardly pointed hairs at the throat of dilated part, blotched with reddish-brown spots at the throat outside, greyish-green otherwise, deep purple within, yellow light windows at dilated part; lobes 1-1.2 cm long, connate at tip forming sub-globose cage, hairy along margins and throat within, trichomes 6-8 $\mathrm{mm}$ long, ovate-oblong, completely reflexed on their back, colour variable, green to white with variously blotched with purple spots. Corona bi-seriate; outer of 5 deeply bifid or 10 equal lobes, c. $3 \times 3 \mathrm{~mm}$ across, equal to the inner corona, ciliate within in two rows, yellow-cream coloured, upper and basal part blotched with purple; inner $3 \mathrm{~mm}$ long, erect, linear, upper and basal part purple, connivent at apex. Pollinarium erect; pollinia yellow-red with pellucid margins, attached to brown-red corpusculum by short caudicle. Follicles 15-20 cm long, glabrous. Seeds comose, 7-10 mm, brownish, marginate; coma silky white.

Flowering \& fruiting: Throughout the year.

Chromosome number: $2 n=22$ (Gosavi et al., 2012); $2 n=66$ (Navaneetham \& Sampathkumar, 1984).

Habitat: Grows along roadsides in wet evergreen forests.

Distribution: South India and Sri Lanka.

Specimens examined: INDIA, Karnataka, Mysore, Dimba, Male Mahadeshwara hills, 22.07.1978, T.M. Ramakrishna TMR 1211 (MH!). Kerala, 

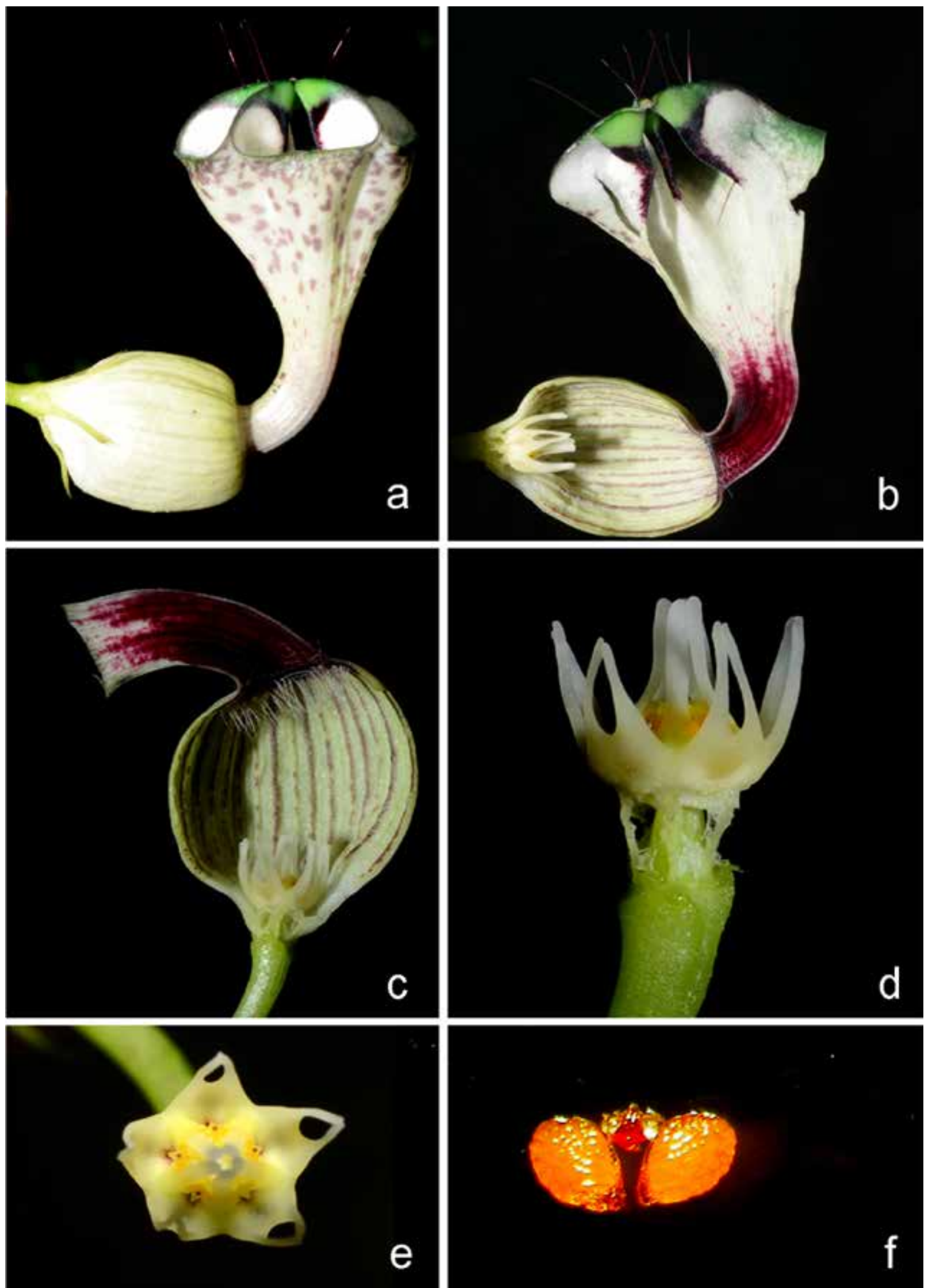

Fig. 40. Ceropegia elegans Wall.: a. Flower; b \& c. Flower L.S.; d. Corona-lateral view; e. Corona-top view; f. Pollinarium. 
Idukki district, Panthanthode, 11.10.1979, s.d., s.coll. s.n. (KFRI!), Kannur district, Chandanathode, 13.07.1978, V.S. Ramachandran 57596 (MH!); Palakkad district, Arurampara slopes, 06.12.1980, N.C. 69151 (MH!); Wayanad district, s.loc., 1921, J.S. Gamble s.n. (MH32741!). Tamil Nadu, Coimbatore district, Anaimalai hills, 25.04.1963, C.P. Sreemadavan CP 571 (MH); Kovai Kutralam, 11.1884, J.S. Gamble s.n. (BSI); Ibid., 08.03.2010, S.S. Kambale, A.R. Kulavmode \& R.V. Gurav SUK 2549 (SUK!); Madurai district, Karanthamalai, 15.02.1958, K. Subramanyam 5322; Kotagiri, Shola near Kodanad view point, 06.05.1971, E. Vajravelu 38286; Kothagiri, Aravenu, 21.11.2012, S.S. Kambale E S.R. Yadav SSK 27 (SUK!); Marappalam, Burliar, 04.12.1971, N.C. Rathakrishnan 39148; above Pykara falls, 12.09.1930, V. Narayanswami 4293 (SUK); Nilgiri district, s.loc., 09.1885, J.S. Gamble 16933; Bikkalli, 5. 1889, J.S Gamble 20710 (BSI!); Tirunelveli district; Courtallum, Palaruvi, 19.03.1958, K. Subramanyam 5586 (MH!); Kalakkad Mundanthurai Tiger Reserve, way to Vistarmottai peak, 12.09.2014, S.S. Kambale SSK 306 (SUK!); Kannikatty Reserve Forest, 18.09.1988, R. Gopalan 88669; Naterikal, 10.03.1963, J. Joseph 15892 (MH!).

Conservation status: Plants of this species are locally abundant. Due to inadequate data it is evaluated as Data Deficient (DD).

Notes: Ceropegiaelegans was discovered by Venerable Archdeacon Hawtayne, in Nilgiri ranges, who sent it to East India Company's Botanic Garden, Kolkata in 1824. Later, Nathaniel Wallich took the living plants to Kew and the plant flowered there was illustrated by Hooker in Curtis's Botanical Magazine (Hooker, 1830). However, he ascribed the name to Wallich by citing 'Wall. MSS' after the diagnosis, who actually provided the original name and a description.

It is a highly variable species showing considerable variations in its leaf shape, colouration, shape of corolla and distribution of trichomes on the lobes. The colour of the corolla lobes and tube may vary from white, yellow, green to red and blotched with brown spots (Fig. 41). This taxon closely resembles C. gardneri Thwaites, C. decaisneana Wight, C. thwaitesii Hook. and C. maculata Bedd. in having ring of hairs at the throat of corolla tube. However, it differs from these species in having equal outer and inner corona lobes and corolla.

Ceropegia gardneri Thwaites, Enum. Pl. Zeyl. 3: 199. 1860; Hook.f., Fl. Brit. India 4: 69. 1883; Trimen, Handb. Fl. Ceylon 3: 165.1895; Kambale \& S.R.Yadav, Asklepios 115: 33. 2013.

C. elegans Wall. var. gardneri (Thwaites) H. Huber, Mem. Soc. Broter. 12: 73. 1957; H. Huber, Dassan. \& Fosberg, Rev. Handb. Fl. Ceylon 4: 119. 1983. Type: Sri Lanka, s.loc., s.d., Gardner C.P. 2838 (holo PDA image!)

C. elegans auct. Ansari, Fasc. Fl. India 16: 14. 1984, non Wallich, 1830.

Fig. 42

Perennial twining herbs. Rootstock fibrous or fascicled. Stem c. $4 \mathrm{~m}$ long, branched, glabrous. Lamina 3.6-8.7 × 1.0-4.5 cm, gradually decreasing in size upwards, ovate-lanceolate, acuminate at apex, rounded-acute at base, ciliolate along margins and nerves beneath, glabrous otherwise; petioles 1-2.5 cm long, channeled above, ciliate along the margins and beneath; Cymes 2-3-flowered, extra-axillary; peduncles $c .7 \mathrm{~mm}$ long, terete, glabrous; bracts 2-25 cm long, linear-subulate; pedicels $c .1 .4 \mathrm{~cm}$ long. Sepals $5-6 \times c .1 \mathrm{~mm}$, subulate, sparsely hairy along the midnerve, glabrous otherwise. Corolla 3-4.2 $\mathrm{cm}$ long, white, blotched with purple; tube $2-3 \mathrm{~cm}$ long, slightly curved, dilated at base, cylindrical at middle, funnel-shaped at throat, glabrous, ring of downwardly directed hairs present at the mouth of dilated part of tube; hairs c. $2 \mathrm{~mm}$ long, unicellular conical, striated with deep purple lines within, colourless to deep purple, lobes $c .1 .5 \mathrm{~cm}$ long, 1.5$2.5 \mathrm{~cm}$ across, connate at tips forming ellipsoid cage, sparsely hairy at the tip and at the inner side, hairs c. $4 \mathrm{~mm}$ long, variously coloured, upper $1 / 3^{\text {rd }}$ green with purple margins, white blotched with purple veins at the middle. Corona bi-seriate, c. $4.5 \times 3.5$ cm; outer of 5-bifid lobes, saucer-bowl shaped, c. $3 \times$ $3.5 \mathrm{~mm}$, shorter than the inner, ciliate within; inner erect, c. $2.5 \mathrm{~cm}$ long, connivent, glabrous, yellow, blotched with purple at the base. Pollinarium c. 0.25 $\times 0.35 \mathrm{~mm}$. Follicles c. $17.5 \mathrm{~cm}$ long, terete.

Flowering: August-December.

Habitat: Plants grows along streams in the lateritic hill slopes in association with Disperis zeylanica Trimen, Ficus scandens Roxb., etc.

Distribution: India (Karnataka) and Sri Lanka. Earlier it was reported to be endemic to Sri Lanka 

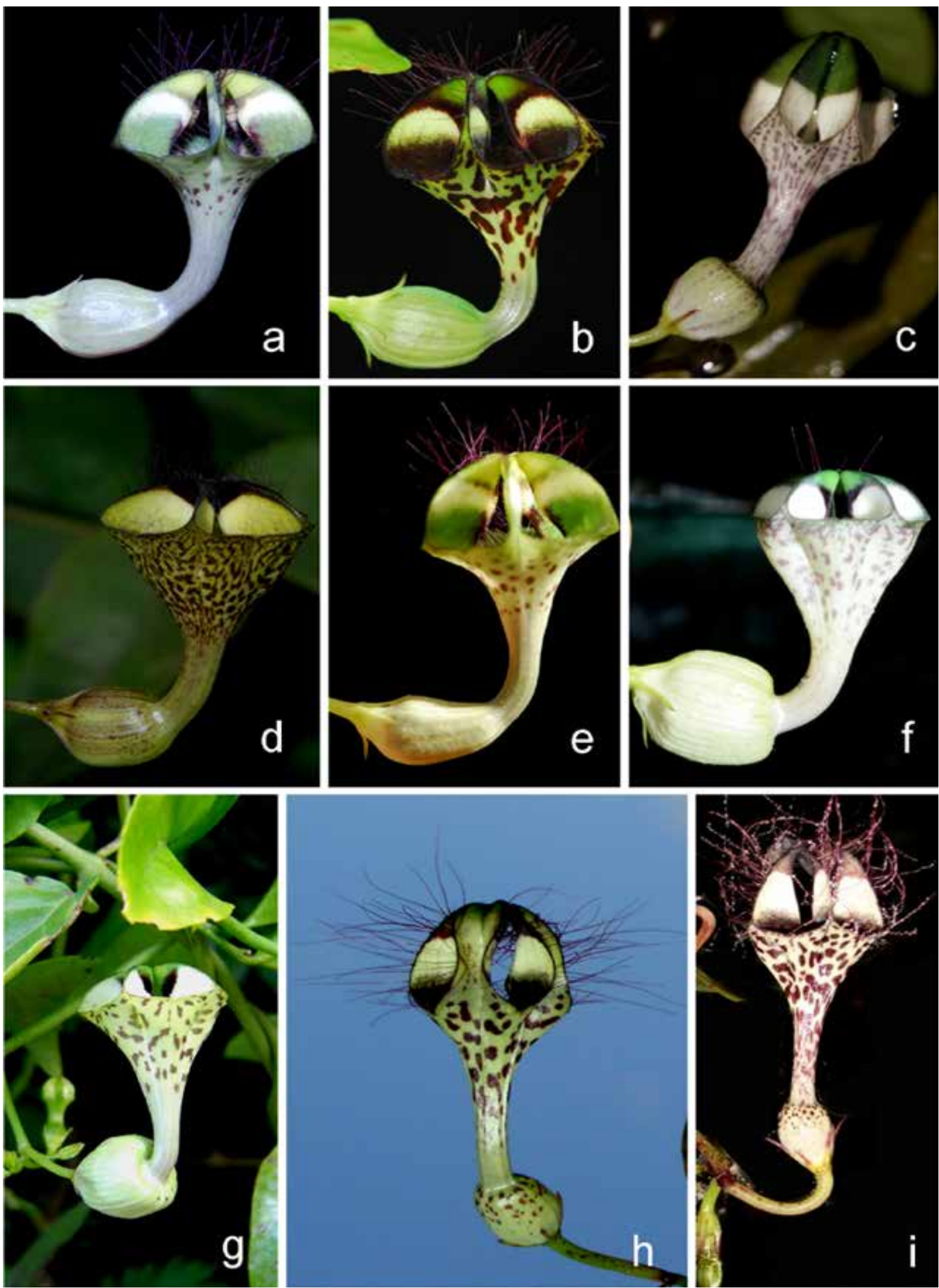

Fig. 41. Floral variations in Ceropegia elegans Wall.: a \& b. Corolla lobes with cilia at the tip; $\mathbf{c} \& \mathbf{g}$. Corolla lobes without cilia; $\mathbf{d}$. Corolla extremely blotched within upper-half of the tube; $\mathbf{e}$. Dilated portion of the tube ovoid; $\mathbf{f}$. Dilated part of the tube globular; $\mathbf{h}$ \& $\mathbf{i}$. Cilia longer in length. 

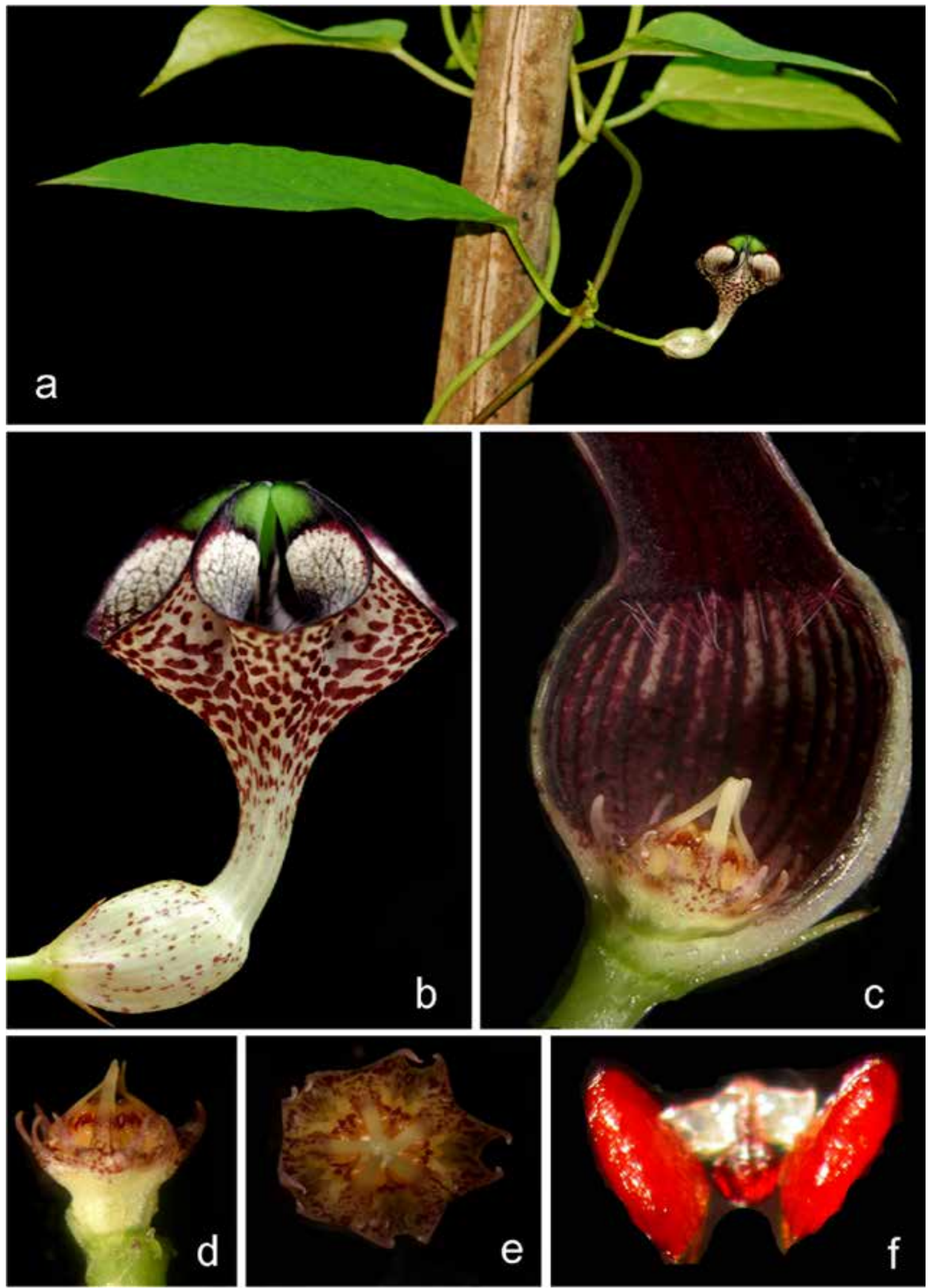

Fig. 42. Ceropegia gardneri Thwaites : a. Habit; b. Flower; c. Flower L.S.; d. Corona-lateral view; e. Corona-top view; f. Pollinarium. 
(Huber, 1983; Bruyns, 1997). However, in the present study it has been collected from a few localities in South India (Karnataka) which forms a new distributional record for the species outside its type locality.

Specimens examined: INDIA, Karnataka, Chikmagalur district, Baba Budan Hills, 15.08.2010, S.S. Kambale \& S.R. Yadav SUK 2554; Ibid., 10.08.2011, S.R. Yadav \& S.S. Kambale SUK 2555; 26.01.2012, S.S. Kambale 5277; Ibid., 16.02.2013, S.R. Yadav \& S.S. Kambale SSK 30 (SUK!).

Conservation status: Nayar and Sastry (1987) assessed the species as Vulnerable due to habitat loss. It is collected from a single locality in India and its presence outside this area is not known. The type collection is from Sri Lanka. Therefore it is treated as Data Deficient (DD).

Notes: Ceropegia gardneri is similar to highly variable $C$. elegans in having fibrous roots and 1-3-flowered cymes and often treated as one of the variants of the latter. Huber (1957) treated it as a variety under the latter, while Ansari (1984) recognized them as conspecific. However, this species can be distinguished by its linear-lanceolate leaves, corolla lobes sparsely ciliate at apex, corolla tube throughout purple within, outer corona shorter than inner and reddish pollinia. Based on these observations Kambale and Yadav (2012) resurrected this species from the synonymy of C. elegans Wall. Ansari (1984) cited C. gardneri as C. gardneri Thwaites ex Hook.f. The species was validly described by Thwaites (1860), the use of "ex" is incorrect. Hence, the species has to be cited as C. gardneri Thwaites.

Ceropegia intermedia Wight, Icon. Pl. Ind. Orient. 4: t. 1263. 1848 emend H. Huber, Mem. Soc. Brot. 12: 61. 1957; Hook.f., Fl. Brit. India 4: 71. 1883, p.p., Gamble, Fl. Madras 4: 858. 1921; K.M. Matthew, Fl. Tamil Nadu Carnatic 2: 937. 1983; B.D. Sharma et al., Fl. Karnataka, Analysis 166. 1984; Ansari, Fasc. Fl. India 16: 17. 1984; S.R. Sriniv. in Henry et al., Fl. Tamil Nadu, Ser I, Analysis 2: 83. 1987; Manilal, Fl. Silent Valley 177. 1988; H.O. Saxena \& Brahmam, Fl. Orissa 2: 1088. 1995; K.M. Matthew, Fl. Palni Hills, S. India 2: 803. 1999; A.P. Jagtap \& N.P. Singh, Fasc. Fl. India 24: 225. 1999; T.S. Nayar et al., Fl. Pl. Kerala 85. 2006;
Karthik. et al., Fl. Pl. India 1: 162. 2009. Lectotype (designated by Ansari, 1984): INDIA, Tamil Nadu, Dindigul district, Serramalie (Sirumalai), s.d., R. Wight s.n. (K000857809 image!). $\quad$ Fig. 43

Perennial glabrous twining herbs. Rootstock tuberous with small fascicled or fibrous roots. Stem c. $2 \mathrm{~m}$ long, slender, branched, glabrous. Lamina 4-11 × 1.2-3.5 cm, ovate-lanceolate, acuteacuminate at apex, rounded at base, hairy along margins and nerves beneath, glabrous otherwise, deep green above, pale below; petioles $1-1.8 \mathrm{~cm}$ long, channeled above, ciliolate along margins, glabrous otherwise. Cymes 2-5-flowered, extraaxillary; peduncles c. $1.5 \mathrm{~cm}$ long, glabrous; pedicels 1.5-1.7 cm long, glabrous. Sepals c. $4 \mathrm{~mm}$ long, more than half the dilated portion of tube, subulate, glabrous, purple tinge at apex. Corolla 1.9-2.6 cm long, purple; tube 1.5-2 cm long, curved, abruptly dilated at base, narrow at the middle, funnel-shaped at mouth, glabrous, greenish at the dilated base, purple otherwise; lobes 4-8 $\mathrm{mm}$ long, ovate-lanceolate, connate at tip forming ellipsoidovoid cage, lobes folded on their back, connate at middle, deep purple and hairy throughout at upper-half, faint at lower half. Corona bi-seriate; outer of 5 entire lobes, c. $1.5 \times 2 \mathrm{~mm}$, glabrous; inner of 5 erect, c. $1.5 \mathrm{~mm}$ long, spathulate-clavate lobes, opposite with outer corona. Pollinium c. 0.2 $\times 0.4 \mathrm{~mm}$. Follicles $9.5-14 \mathrm{~cm}$ long, erect, tapering towards both ends, glabrous.

Flowering \& fruiting: June-January.

Habitat: Grows along slopes, road-sides in evergreen forests.

Distribution: India (Kerala, Orissa, Tamil Nadu).

Specimens examined: INDIA, Kerala, Idukki district, Kulamavu, 03.10.1983, C.N. Mohanan 79958 (CAL!); Palakkad district, way to Anavai, 09.11.1976, E. Vajravelu 48900 (CAL!); Thiruvananthapuram district, Bodimettu, 12.1910, A. Meebold 13735 (CAL!). Tamil Nadu, Coimbatore district, Sholayar, 26.12.1963, K. Ramamurthy 18112 (MH!); Dindigul district, Kodaikanal, 10.1946, J. Pallithanam 1995 (RHT!);

Kanyakumari district, Muthulkunzhi, 09.09.1976, A.N. Henry 48313 (CAL!); Palni hills, 1897, A.G. Bourne 329 (CAL!); Tirunelveli district, Sengaltheri, 19.09.1967, E. Vajravelu 29157 (MH!). 

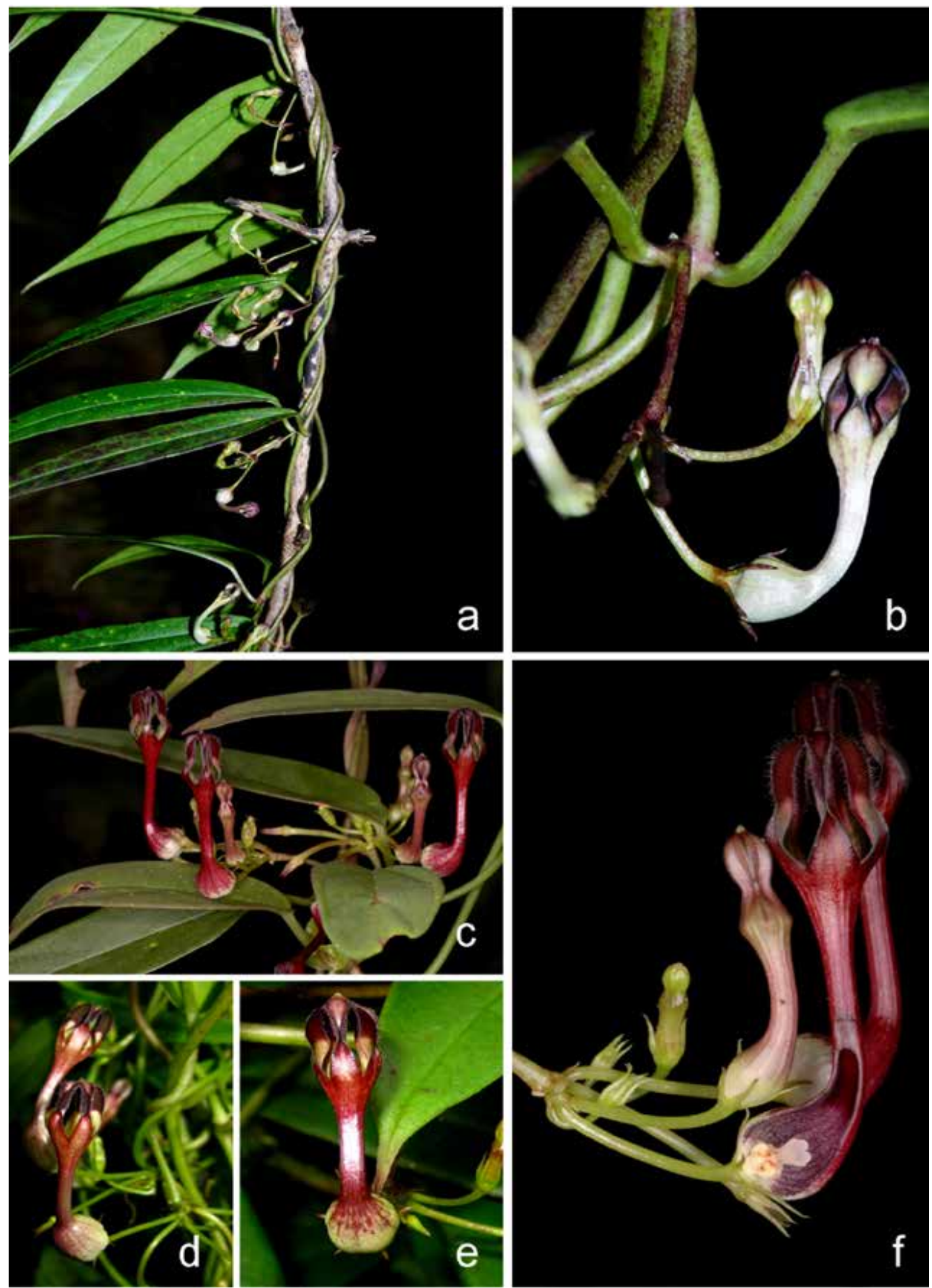

Fig. 43. Ceropegia intermedia Wight: a. Habit; b-d. Inflorescences; e. Flower; f. Flower L.S. (Photos Navendu Page). 
Conservation status: It is assessed as Vulnerable [VN: B2 b (iii, v), c (iii, iv)].

Notes: Wight (1848) described C. intermedia based on the specimens collected from Sirumalai, Dindigul in Tamil Nadu. As opined by Huber (1957), this species is often confused with $C$. candelabrum. The outer corona (Wight's illustration t. 1263) is completely glabrous rather than hairy at the base as stated in the description. Huber clarified the ambiguity in the circumscription and emended the description.

Ceropegia maculata Bedd., Madras J. Lit. Sci. Ser. 3(1): 52. 1864; Gamble, Fl. Madras 4: 859. 1921; H. Huber, Mem. Soc. Brot. 12: 176. 1957; Ansari, Fasc. Fl. India 16: 23. 1984; S.R. Sriniv. in Henry et al., Fl. Tamil Nadu Ind. Ser. I. Analysis 2: 84. 1987; M.P. Nayar \& Sastry, Red Data Book Indian Pl. 2: 46. 1988; A.P. Jagtap \& N.P. Singh, Fasc. Fl. India 24: 223. 1999; T.S. Nayar et al., Fl. Pl. Kerala 86. 2006; Karthik. et al., Fl. Pl. India 1: 162. 2009. (designated by Kambale \& Yadav, 2015): INDIA, Tamil Nadu, Anaimalais (Anamallays), s.d., R.H. Beddome (CAL0000018018).

Fig. 44

Perennial twining herbs. Rootstock with fibrous roots. Lamina $5.2-9.5 \times 1.7-5 \mathrm{~cm}$, ovatelanceolate, acute-acuminate at apex, narrow at base, with glands at the base of lamina, punctate, glabrous above, ciliolate along nerves beneath and along margins; petioles $1.5-3.5 \mathrm{~cm}$ long, channeled above, pilose along margins. Cymes sub-umbellate, 7-15-flowered, extra-axillary; peduncles 1.4-2.5 cm long, slender, glabrous; pedicels $0.9-2 \mathrm{~cm}$ long. Sepals 2-5 mm long, subulate, glabrous. Corolla $1.9-2.1 \mathrm{~cm}$ long, pale greenish-purple; tube 1.3$1.6 \mathrm{~cm}$ long, curved, dilated at base, ring of hairs at throat within; lobes 3-5 mm long, oblong or oblong-lanceolate, connate at tip forming ovoid cage, hairy, bluish-green. Corona bi-seriate; outer of 5 deeply bifid lobes, connate at tip, as long as inner, hairy at base; inner of 5 erect lobes, alternate with outer corona. Pollinarium c. $0.4 \times 0.4 \mathrm{~mm}$. Follicles 10-20 cm long, terete, thin.

Flowering \& fruiting: June-February.

Habitat: Species found growing along shady slopes in forests.

Distribution: Endemic to Kerala and Tamil Nadu, India.
Specimens examined: INDIA, Kerala, Palakkad district, Palakkad, 02.09.2014, P. Kumar SSK 301 (SUK!); Thiruvananthapuram district, Naduvengad, 29.11.1905, C.A. Barber 7166 (MH!); Thrissur district, Peechi, 14.11.1989, N. Sasidharan 5514; Sholayar forest, 19.09.1983, N. Sasidharan 2954 (KFRI!). Tamil Nadu, Anaimalais hills, s.d., R.H. Beddome 26 (CAL!).

Conservation status: Nayar and Sastry (1987) assessed it as Endangered or possibly extinct however it has been collected from few places of South India. It is assessed as Critically Endangered [CR: B2 a, b (iii, iv, v), c (iv)].

Notes: Ansari (1984) treated C. maculata conspecific to C. parviflora Trimen, however, after examining the coloured drawing of C. parviflora by Trimen and critical analysis of the specimens, it is revealed that the former is distinct in having leaves narrow at the base, hairy corolla lobes and linear inner corona lobes (vs. leaves subcordate at the base, glabrous corolla lobes and spathulate inner corona lobes).

Ceropegia manoharii Sujanapal, P.M. Salim, Anil Kumar \& Sasidh., J. Bot. Res. Inst. Texas 7(1): 342. 2013. Type: INDIA, Kerala, Wayanad district, Meppadi forests, 10.12.2009, P. Sujanapal \& P.M. Salim MSSH 0403 (holo MH!; iso CALI!, KFRI!).

Fig. 45

Perennial twining herbs. Rootstock with fascicled, fleshy roots. Stem terete, branched, glabrous. Lamina 5-10 × 2-3.5 cm, thickly coriaceous, ovate-lanceolate, acute at apex, cuneate-narrow at the base, glabrous-ciliolate, dark green-shining above, pale green below; petioles 8-15 mm long. Cymes 1-2-flowered, extra-axillary; peduncles 8-12 mm long, glabrous; bracts solitary, 2-4 mm long, linear, glabrous; pedicels $1.6-2.9 \mathrm{~cm}$ long, glabrous. Sepals 3-5 mm long, linear, glabrous. Corolla 3-4 cm long; tube 1.4-1.8 cm long, dilated at base, cylindrical at middle, sub-cylindrical at throat, ring of downwardly pointed hairs at the mouth of dilated part, greenish-yellow, striated with purplish-brown on the upper-half and deep purple at lower half within; lobes 1.6-2.2 cm long, completely folded back, oblong, connate at tip forming an ovoid cage, hairy along margins and at the tip and at the base within, white at lower half, deep green at upper-half. Corona bi-seriate; outer 

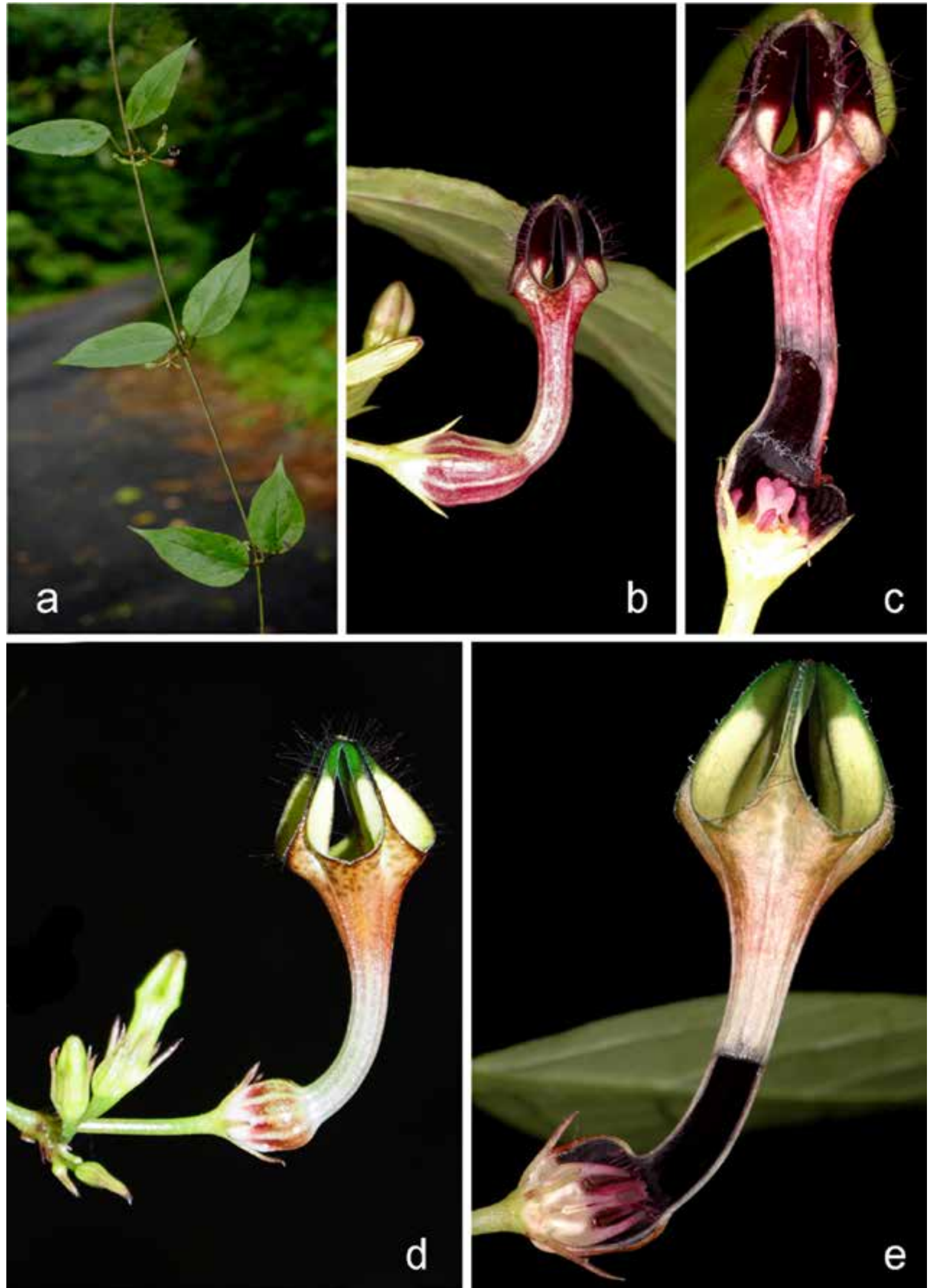

Fig. 44. Ceropegia maculata Bedd.: a. Habit; $\boldsymbol{b}$ \& d. Flower; $\mathbf{c}$ \& e. Flower L.S. (Photos Navendu Page). 
of 5 deeply bifid connivent lobes, saucer-shaped, c. $1.5 \mathrm{~mm}$ long, densely hairy along margins and within; inner of 5 linear, connivent, purple lobes, c. $2.5 \mathrm{~mm}$ long, incumbent on gynostegium. Pollinarium c. $0.3 \times 0.25 \mathrm{~mm}$. Follicles in pairs, unequal, 15-18 cm long, glabrous; seeds c. $5 \times 2$ $\mathrm{mm}$, ovate-oblong, black, comose; coma 8-15 mm long, silky white.

Flowering \& fruiting: August-February.

Habitat: Grows in grasslands at an elevation of 1500-1850 m.

Distribution: Endemic to Kerala, India.

Conservation status: While describing the species two authors counted only 44 mature individuals from the type locality. At present the species is assessed as Data Deficient (DD).

Notes: Ceropegia manoharii is allied to C. elegans but differs in having densely hairy outer corona and corolla tube sub-cylindrical at throat.

Ceropegia metziana Miq., Anal. Bot. Ind. 3. 11. 1852; Hook.f., Fl. Brit. India 4: 74. 1883; Gamble, Fl. Madras 4: 859. 1921; Ansari, Fasc. Fl. India 16: 25. 1984; S.R. Sriniv. in Henry et al., Fl. Tamil Nadu Ind., Ser I: Analysis 2: 84. 1987; M.P. Nayar \& Sastry, Red Data Book Indian Pl. 2: 51. 1988; A.P. Jagtap \& N.P. Singh, Fasc. Fl. India 24: 232. 1999; T.S. Nayar et al., Fl. Pl. Kerala 86. 2006; Karthik. et al., Fl. Pl. India 1: 163. 2009. Neotype: INDIA, Tamil Nadu; Nilgiris district, Devala, s.d., J.S. Gamble 15601 (K000014449 image!).

Ceropegia gracilis Bedd., Madras J. Lit. Sci. Ser. 3 (1): 53. 1864. pr. max. p. Type: Not located or apparently none preserved.

Ceropegia oculata auct. H. Huber, Mem. Soc. Brot. 12: 64. 1957, non Hook., 1844.

Fig. 46

Perennial twining herbs. Stem glabrous throughout. Lamina $6.8-15.5 \times 3.5-6.5 \mathrm{~cm}$, ovate-lanceolate, acute at apex, rounded at base, pubescent above and nerves beneath, ciliate along margins, glabrous otherwise, deep green above, pale below; petioles 1.5-1.8 cm long, pilose. Cymes 2-7-flowered, extra-axillary; peduncles $2.5-4.5 \mathrm{~cm}$, glabrous; pedicels $2.5-3.0 \mathrm{~cm}$, glabrous. Sepals $0.6-1 \mathrm{~cm}$ long. Corolla 4-7.7 cm long, greyish dotted with purple or pink dots; tube $2-3.7 \mathrm{~cm}$ long, dilated at base, narrow at middle, funnel-shaped at throat, glabrous within except for ring of hairs at the throat of dilated base, pink at the base, with pinkish striations from below upwards; lobes 2-4 cm long, ovate-deltoid at base, narrow at middle, linearspathulate at apex, coherent at middle, connate at tip forming ovoid cage, dull pink. Corona biseriate; outer of 5 notched lobes at tip, as long as or little shorter than inner corona, saucer-shaped, hairy along margins and within, yellow at base, red along margins; inner of 5 erect lobes, red at lower half, pink at upper-half, glabrous. Fruits not observed.

Flowering \& fruiting: September-December.

Habitat: Grows along roadsides and open places in evergreen forests.

Distribution: South India (Karnataka, Kerala, Tamil Nadu).

Specimens examined: INDIA, Kerala, Idukki district, Kakki dam, 10.10.1983, A.G. Pandurangan 79294 (MH!); Kollam district, Pamba dam, 30.09.1976, K. Vivekananthan 48341 (MH!); Pathanamthitta district, Veluthodu forest, 21.10.1989, $R$. Chandrasekharan 89367 (MH!); Thrissur district, Karimala hills, 23.10.1990, N. Sasidharan 5729; Peechi, 18.09.1990, N. Sasidharan 5714; Sholayar forest, 25.10.1992, N. Sasidharan 5933 (KFRI!). Tamil Nadu, Coimbatore district, Siruvani, 31.12.1960, J. Joseph 11741; (MH!); Nilgiris district, Rughivayal reserve forest, 26.11.1972, E. Vajravelu 43493 (MH!).

Conservation status: Nayar and Sastry (1987) assessed it as Rare.

Notes: Ceropegia metziana closely resembles C. decaisneana Wight in its twining habit, but differs in having tuberous roots, slightly notched outer corona and linear corolla lobes. Huber (1957) considered it as conspecific to C. oculata Hook., but differs in having longer and linear corolla lobes, slightly notched outer corona and glabrous pedicel and peduncle.

Ceropegia schumanniana Swarupan. \& Mangaly, Nordic J. Bot. 12(6): 699. 1992; Karthik. et al., Fl. Pl. India 1: 163. 2009. Type: INDIA, Tamil Nadu, Coimbatore district, Kharian Shola, Near Top Slip, 29.12.1976, K. Swarupanandan 13911 (MH: not found or apparently none preserved). Lectotype 

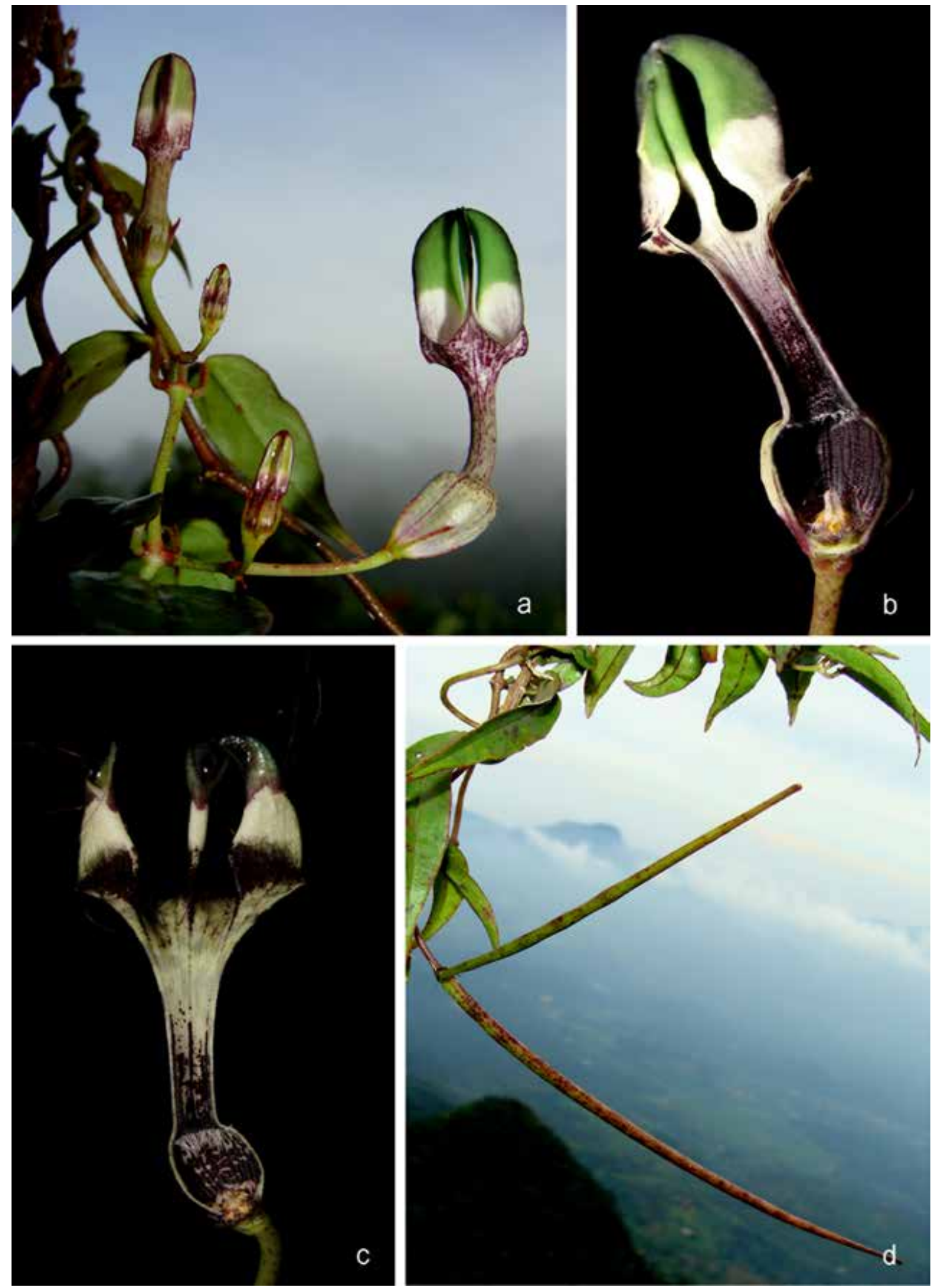

Fig. 45. Ceropegia manoharii Sujanapal, P.M. Salim, Anil Kumar \& Sasidh.: a. Habit; b \& c. Flower L.S.; d. Follicle (Photos Dr. P. Sujanapal). 

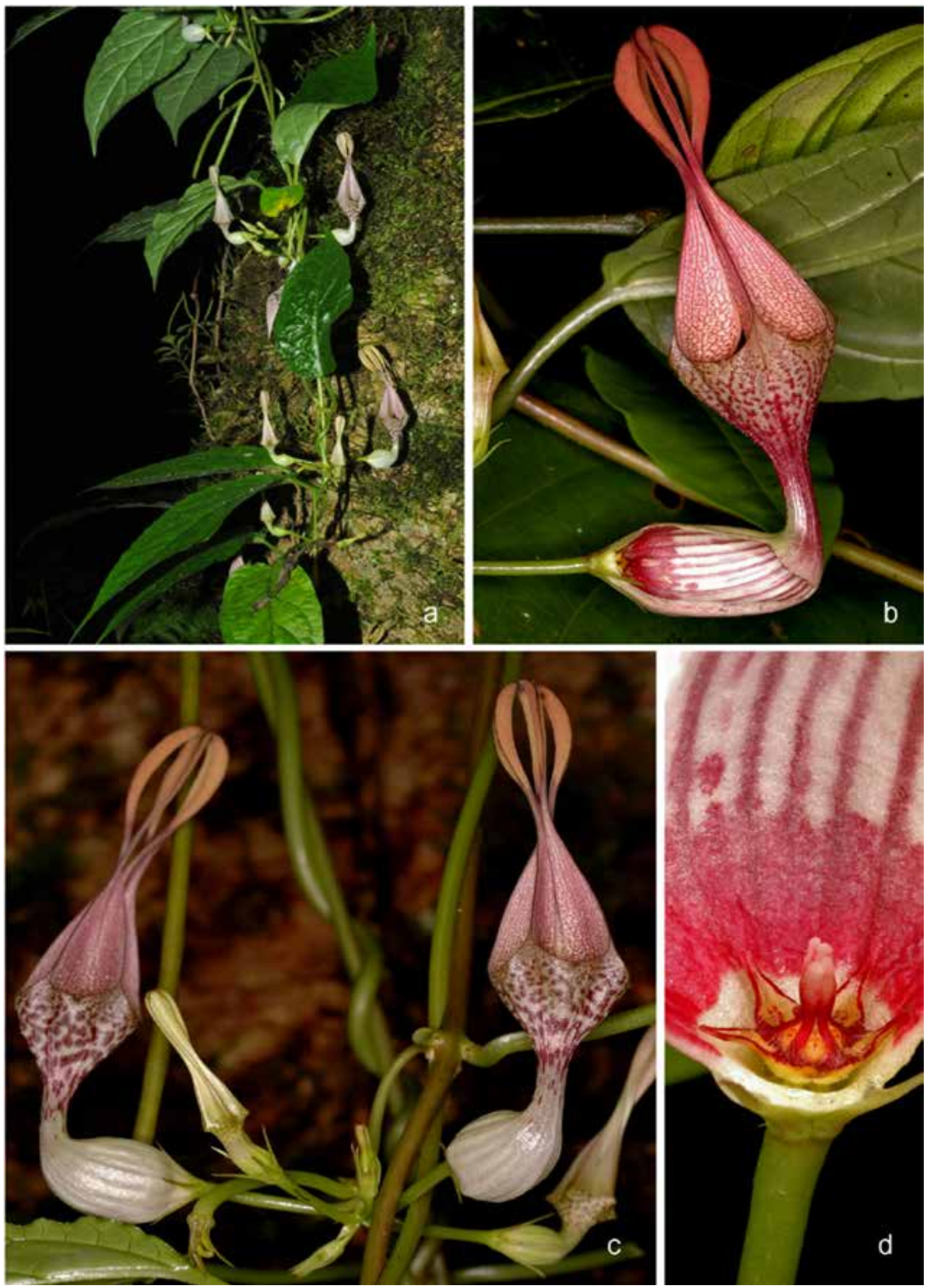

Fig. 46. Ceropegia metziana Miq.: a. Habit; b. Flower L.S.; c. Inflorescence; d. Corona (Photos Navendu Page). 
(designated here): Fig. 3. Nordic J. Bot. 12(6): 700. 1992.

Perennial trailing to twining glabrous herbs. Lamina 4-4.5 $\times 1.5-2 \mathrm{~cm}$, ovate-lanceolate, acuminate at apex, narrow at base, glabrous, deep green with few purple spots above, pale beneath; petioles 0.8-1.6 $\mathrm{cm}$ long, channeled above, ciliolate along margins, glabrous otherwise. Cymes 5-7-flowered, extraaxillary; peduncles $4-7 \mathrm{~mm}$ long, glabrous; bracts and bracteoles linear, glabrous; pedicels $6-7 \mathrm{~mm}$ long, glabrous. Sepals 4-4.5 mm long, subulate, glabrous. Corolla 1.2-1.5 cm long; tube $0.8-1$ $\mathrm{cm}$ long, curved, dilated at base, sub-cylindricalfunnel-shaped at throat, glabrous except for ring of hairs at the throat, deep purple within; lobes 3-4 mm long, elongate-ovate, completely folded on their back, connate at tip forming ovoid cage, hairy along margins. Corona shortly stipitate, bi-seriate; outer deeply bifid, shorter than inner corona, linear, hairy within, pink at upper-half, yellow at lower half; inner erect, linear-clavate, pink. Fruits not observed.

Flowering: December.

Habitat: Grows along edges of evergreen forests.

Distribution: Only known from type locality, Tamil Nadu, India. This species has never been collected after type.

Conservation status: After type it has not been collected and is not represented at any Indian herbaria. It is assessed here as Data Deficient [DD].

Notes: Ceropegia schumanniana has been accommodated in the C. sect. Janthina H. Huber because of the presence of a ring of hairs at the throat of dilated base and undivided erect inner coronal lobes. Five other species belonging to this section are known from India, viz. C. decaisneana Wight, C. thwaitesii Hook., C. elegans Wall., C. maculata Bedd. and recently described C. manoharii from Kerala. The mouth of the corolla tube is funnel-shaped and the lobes of the outer coronal scales are deltoid and hairy or puberulent in first three species. C. schumanniana differs from these species in the sub-cylindrical throat of the corolla tube, and an outer corona with glabrous, narrow lobes shorter than inner.

Swarupanandan and Mangaly (1992) mentioned only a single specimen in the protologue as type deposited at MH. However, authors could not locate the type material. Therefore, the figure which is the only original material, has been designated here as the lectotype following Art. 9.3 of Shenzhen Code (Turland et al., 2018).

Ceropegia thwaitesii Hook., Bot. Mag. 80: t. 4758. 1854; Hook.f., Fl. Brit. India 4: 71. 1883; Trimen, Handb. Ceylon Pl. 3: 166. 1895; Fyson, Fl. Nilgiri \& Pulney 3: 81. t. 426. 1920; Gamble, Fl. Madras 4: 858. 1921; H. Huber, Mem. Soc. Brot. 12: 71. 1957; Ansari, Fasc. Fl. India 16: 30. 1984; M.P. Nayar \& Sastry, Red Data Book Indian Pl. 2: 58. 1988; K.M. Matthew, Fl. Palni Hills, S. India 2: 803. 1999; A.P. Jagtap \& N.P. Singh, Fasc. Fl. India 24: 240. 1999; T.S. Nayar et al., Fl. Pl. Kerala 87. 2006; Karthik. et al., Fl. Pl. India 1: 164. 2009. Lectotype (designated by Ansari 1984): Bot. Mag. t. 4758. 1854.

Perennial, extensive twining herbs. Stem branched, glabrous, slender. Lamina $2.2-11 \times 1-5.5 \mathrm{~cm}$, ovate-lanceolate, acute at apex, rounded-cordate at base, ciliate along margins, glabrous otherwise; petioles $0.5-3.5 \mathrm{~cm}$ long, channeled above, glabrous. Cymes sub-umbellate, 2-6-flowered, extra-axillary; peduncles 1-2.5 cm long, glabrous; pedicels $2.5-4 \mathrm{~cm}$ long, glabrous. Sepals $5-7 \mathrm{~mm}$ long, subulate, glabrous,red at tip. Corolla 3-6.7 cm long; tube $3.5-4.2 \mathrm{~cm}$ long, slightly curved, abruptly dilated at base, narrow at middle, funnelshaped at throat, glabrous within except for ring of hairs at the throat of dilated part, yellow blotched with deep red spots at the expanded portion of throat; lobes 1.2-2.5 cm long, ovate-oblong, glabrous, completely folded along their back, glabrous throughout, yellow at lower $2 / 3^{\text {rd }}$ part, transverse band of deep red colour, dull green at upper $1 / 3^{\text {rd }}$ part. Corona bi-seriate; outer bowlshaped, deeply bifid, shorter than inner corona, hairy along margins; inner erect, linear-spathulate, twice as long as the outer corona, glabrous. Follicles 20-26.5 cm long, glabrous. Seeds c. $1 \times 0.3 \mathrm{~cm}$, comose; coma c. $2.5 \mathrm{~cm}$ long, silky white.

Flowering \& fruiting: February-November.

Chromosome number: $2 n=22$ (Navaneetham \& Sampathkumar, 1984).

Habitat: Grows at shola forests in association with Strobilanthes sp. 
Distribution: India (Kerala, Tamil Nadu) and Sri Lanka.

Specimens examined: INDIA, Kerala, Idukki district, Devikolam, Umaiya Malai, 20.04.1966, B.V. Shetty 27345 (MH!), Munnar, Vandaravu road, 13.05.1985, K.M. Matthew 41450 (RHT!); Kasargod district, Ranipuram, Deviar Estate to Naduthottam, Sethur hills, S.R. Srinivasa. 68040 (RHT!).Tamil Nadu, Dindigul district, Palni hills, s.d., s.coll. s.n. (MH!); Ibid., 25.06.1898, Bourne 1134; Kodaikanal, 11.09.1905, C.A. Barber CAB 7251 (MH!); Ibid., 05.1941, s.coll. s.n. (RHT!); Nilgiris, Coonoor, 11.1985, s.coll. s.n. (MH!); Tirunelveli district, Old Courtallum forest, 17.02.1983, E. Vajravelu 76580 (MH!).

Conservation status: It is represented by few specimens in Indian herbaria. Due to insufficient information it is considered as Data Deficient (DD).

Notes: Ceropegia thwaitesii resembles C. cumingiana Decne., but differs from it in having abruptly dilated corolla tube, narrow at the middle and corolla lobes with transverse bands across. Hooker (1854) did not mention any specimen in the protologue but provided a beautiful colour drawing. Huber (1957) cited 'specimen cultum at Herb. K'. This specimen is not a part of original material as its type. Since it is collected after the date of publication, hence cannot serve as type. Ansari (1984) inadvertently cited the coloured drawing as lectotype (Bot. Mag. t. 4758).

\section{Ceropegia sect. Phalaena}

Ceropegia sect. Phalaena H. Huber, Mem. Soc. Brot. 12: 30. 1957; Bruyns, S. African J. Bot. 112: 411. 2017. Type: Ceropegia aristolochioides Decne.

Climbing perennial herbs. Rootstock a small tuber with fibrous roots. Stem succulent, glabrous. Leaves fleshy, subulate to lanceolate, reduced to scale, sessile, caducous, glabrous. Cymes 2-5(-10)-flowered. Corona bi-seriate.

Distribution: Twenty four species in the Africa, southern Arabian Peninsula (including Socotra), southern India and Sri Lanka. Only one species i.e. C. juncea Roxb., is found in India. (Table 1).

Ceropegia juncea Roxb., Pl. Coromandel 1: 12, t.10. 1795; Dalzell \& A. Gibson, Bombay Fl.
153. 1861; Hook.f., Fl. Brit. India 4: 68. 1883; T. Cooke, Fl. Bombay 2: 240. 1905; H. Huber, Mem. Soc. Brot. 12: 61. 1957; K.M. Matthew, Fl. Tamil Nadu Carnatic 2: 937. 1983; B.D. Sharma et al., Fl. Karnataka, Analysis 166. 1984; Ansari, Fasc. Fl. India 16: 18. 1984; S.R. Sriniv. in Henry et al., Fl. Tamil Nadu Ind., Ser I, Analysis 2: 83. 1987; Yadav et al., J. Bombay Nat. Hist. Soc. 86: 481. 1990; Hanumanthappa in Pull. \& Moulali, Fl. Andhra Pradesh 2: 585. 1997; K.M. Matthew, Fl. Palni Hills, S. India 2: 803. 1999; A.P. Jagtap \& N.P. Singh, Fasc. Fl. India 24: 227. 1999; M.R. Almeida, Fl. Maharashtra 3A: 233. 2001; F. Albers \& Meve, Ill. Handb. Succ. Pl. Asclepiadaceae 84. 2002; T.S. Nayar et al., Fl. Pl. Kerala 85. 2006; Karthik. et al., Fl. Pl. India 1: 162. 2009; Kambale \& S.R. Yadav, Asklepios 115: 35. 2013. Lectotype (designated by Huber, 1957): Roxb., Pl. Coromandel 1: 12, t. 10. 1795.

Fig. 47

Vernacular names: Kanvel (Marathi), Jettlli, Jakili (Kannada), Bella gada, Pullam thige (Telugu).

Perennial extensively twining, occasionally repent, succulent herbs, with reduced tubers. Stem c. 3 $\mathrm{m}$ long, succulent, branched, glabrous, reddishgreen. Leaves sessile; lamina 0.8-1.6 × 0.18-0.3 $\mathrm{cm}$, ovate-lanceolate, reduced, scaly, caducous, fleshy, glabrous. Cymes sub-umbellate, 2-5 (-10)-flowered, extra-axillary; peduncles $0.3-1 \mathrm{~cm}$ long, terete, glabrous; bracts $1.6-2 \times 0.25-0.32 \mathrm{~mm}$, subulate, glabrous; pedicels $5-9 \mathrm{~mm}$ long, terete, glabrous. Sepals 5-5.2 mm long, acute, glabrous. Corolla $4.2-6.4 \mathrm{~cm}$ long, yellowish-green; tube $2.8-3.8 \mathrm{~cm}$ long, curved almost right angle to the pedicels, dilated at base, funnel-shaped at throat, hairy within near the throat, ring of hairs at the throat of dilated portion, blotched with purple outside; lobes 5 , linear, $2.2-2.5 \mathrm{~cm}$ long, connate at tip forming conical cage, hairy along margins and within (hairs $5 \mathrm{~mm}$ long, purple) above the middle, greenish-yellow at lower half, dull brownbrown at upper-half. Corona bi-seriate, 5-5.5 $\times$ 4-4.5 mm; outer of 5-bifid lobes, bowl-shaped, ciliate along margins and within; inner of 5 erectodivergent lobes, 3.5-4 mm long, hairy at the base, yellow at the tips, purple otherwise. Pollinarium $c$. $0.3 \times 0.3 \mathrm{~mm}$. Follicles in pairs, usually unequal, $6.7-9 \mathrm{~cm}$ long, acute at apex, slightly curved and swollen at the tips, glabrous. Seeds comose; coma $c$. $2 \mathrm{~cm}$ long, silky white. 

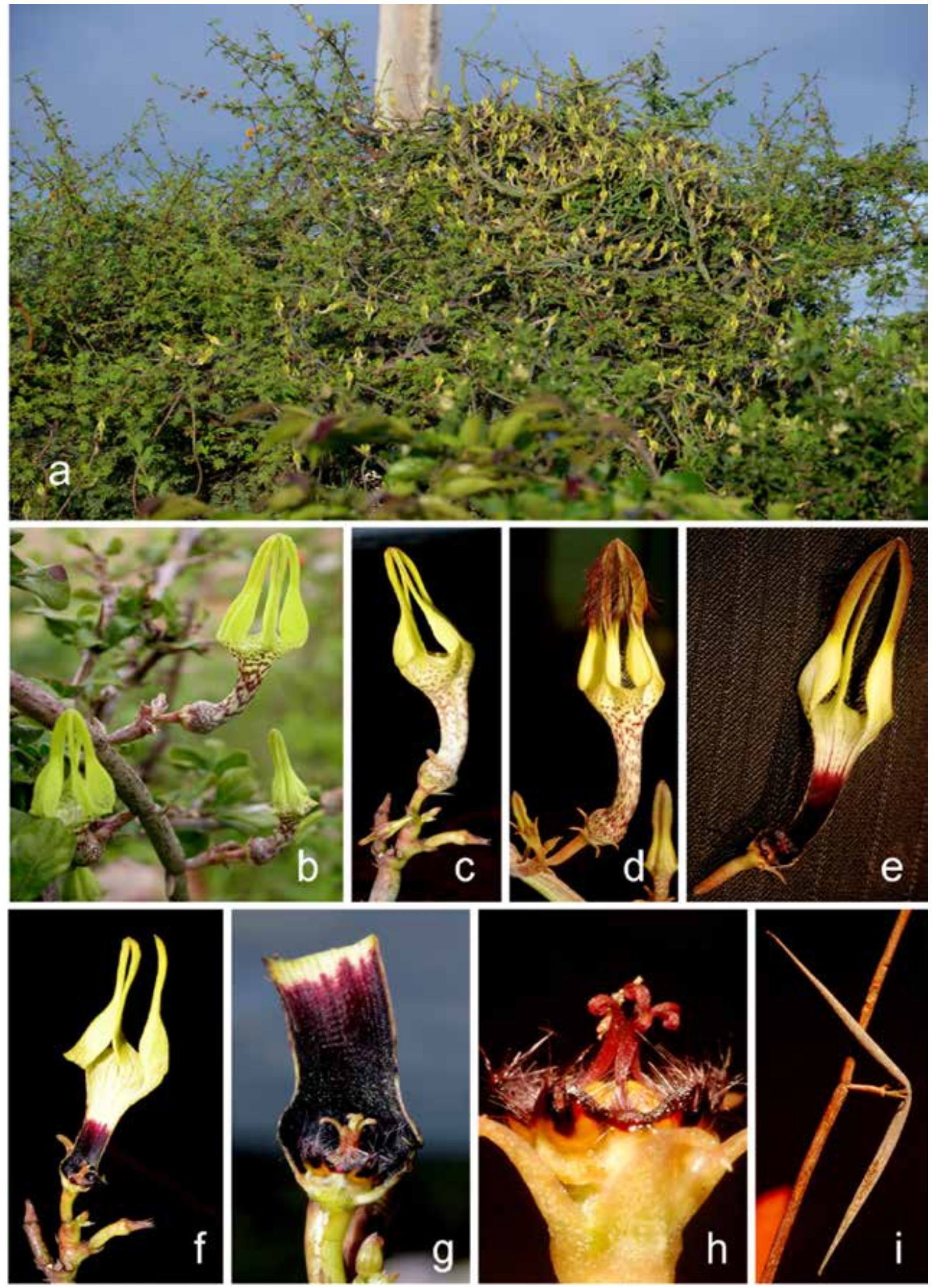

Fig. 47. Ceropegia juncea Roxb.: a \& b. Habit; c \& d. Flower; e-g. Flower L.S.; h. Corona-lateral view; i. Follicle. 
Flowering \& fruiting: July-November.

Chromosome number: $2 n=66$ (Navaneetham, 1981).

Habitat: Occurs in dry deciduous forests in association with Canthium sp., Capparis aphylla, Roth, Prosopis juliflora DC. etc.

Distribution: India (Andhra Pradesh, Karnataka, Kerala, Maharashtra, Tamil Nadu) and Sri Lanka.

Specimens examined: INDIA, Andhra Pradesh, Anantapur district, Kalasamudram Reserve Forest, 13.10.1984, T. Pullaiah \& E. Cheeranjiv 2866; Rayadurg forest, 14.08.2011, B.R. Rao \& A. Narayanaswamy 35550 (SUK!); Chittoor district, Kambakkam, 29.11.2007, M.V. Suresh Babu \& VSR 31147 (SUK!); Prakasam district, Papinenipalli, 06.12.2007, B. Sadasivaiah \& S.K. Basha 30498 (SKU!). Karnataka, Bagalkot district, Badami, 09.1910, A. Meebold11365 (CAL!); Belgaum district, Gokak, 31.08.2010, S.S. Kambale \& A.N. Chandore SUK 2565; Yaragatti, 09.10.2014, S.S. Kambale \& S.R. Yadav SSK 324; Ibid., 24.08.2014, S.S. Kambale E S.R. Yadav SSK 310 (SUK!); Dharwad district, Dharwad, 25.09.1889, W.A. Talbot s.n. (BSI!); East Karnataka, Kamlaruva shola forest, 22.10.1975, N.P. Singh 140841 (BSI!); Hassan district, Belavathally forest, 10.06.1969, C.J. Saldanha 13752 (CAL!). Kerala, Idukki district, Alampatty, Marayur Range, 28.11.1979, Nambiar 1066 (KFRI!). Tamil Nadu, Coimbatore district, Coimbatore, 29.01.1943, s.coll. s.n.; Marudamalai hills, 13.02.2013, S.S. Kambale SSK 29 (SUK!); Nellimalai Reserve Forest, 16.10.1962, K. Ramamurthy 14782; Palamalai, 05.09.1969, M.V. Vishwanathan MVV 104; Pollachi, Padmnabhapuram, 13.09.1976, K. Swarupanandan CU 13679; Thekkumalai, 19.11.1956, K.M. Sebastine 1378; Kamaraj district, Thaniparai, 30.03.1994, S.P. Subramani 48 (FRLH!); Kanyakumari district, Perunchilambu, 08.12.1980, M.S. Swaminathan 68980 (MH!); way to Wattakotta, s.d., S.B., A.F.S.K. \& C.M.U. 18317 (FRLH!); north Arcot district, Karanapoondi village, 07.10.1978, E. Vajravelu 55993 (MH!); Ramanathapuram district, Kamuthakudi, 16.11.1989, V. Balasubramanian 2244; Tirupathur, 20.12.1964, K. Ramamurthy 22804 (MH!); Salem district, Woddapatti Reserve Forest, 28.11.1964, E. Vajravelu 21987 (MH!); South Arcot district, s.loc., 04.09.1899, C.A. Barber 767 (MH!); Tirunelvelli district, Tenkasi, 03.11.2014, S.S. Kambale SSK 331 (SUK!); Tiruvannamalai district, Thanagoundanpudur, 06.09.2001, R.V. Shankar 73579 (FRLH!).

Conservation status: It is a widely distributed species and represented by sufficient number of individuals in all populations. It is assessed here as Vulnerable [VN: B2 b (iii, iv, v) c (iii, iv)].

Notes: The leaves being scaly and caducous often appears leafeless. It is restricted to drier parts of Peninsular India. It closely resembles $C$. distincta N.E. Br., an African species in its succulent habit.

\section{Ceropegia sect. Tiloris}

Ceropegia sect. Tiloris H. Huber, Mem. Soc. Brot. 12: 33. 1957; Bruyns, S. African J. Bot. 112: 429. 2017. Type: Ceropegia attenuata Hook.

ser. Attenuatae H. Huber, Mem. Soc. Brot. 12: 33. 1957. Type: Ceropegia attenuata.

ser. Pusillae H. Huber, Mem. Soc. Brot. 12: 27. 1957. Type: Ceropegia pusilla Wight \& Arn.

Erect slender (except C. arnottiana) tuberous herbs. Leaves linear-lanceolate, subsessile-sessile, glabrous to puberulous. Flowers solitary or in 3-flowered cymes.

Distribution: Thirty eight species in the world distributed in India and southeast Asia, Malaysia (New Guinea, Philippines) to northern Australia. This section is represented by 13 species in India of which 12 are endemic to Peninsular India (Table 1).

Key to the species of sect. Tiloris H. Huber

1. Plants erect …..........................................................

1. Plants climbing ............................. C. arnottiana

2. Flowers solitary .................................................... 3

2. Flowers in cymes …......................................12

3. Corolla lobes shorter than tube ....................... 4

3. Corolla lobes as long as or longer than tube ..9

4. Corolla tube abruptly dilated towards base ... 5

4. Corolla tube gradually dilated towards base ...7

5. Corolla tube cylindrical at throat ....... C. jainii

5. Corolla tube funnel-shaped at throat ..............6

6. Corolla lobes glabrous, not twisted C. concanensis

6. Corolla lobes glandular hairy, twisted 
C. nampyana

7. Corolla lobes beaked / linear .C. mahabalei

7. Corolla lobes otherwise . .8

8. Corolla lobes lanceolate, forming ampuliform globular cage, puberulent, reflexed.

C. mohanramii

8. Corolla lobes spathulate, forming ovoid cage, glabrous, not reflexed C. pusilla

9. Corolla lobes spirally coiled, hairy ..... C. spiralis

9. Corolla lobes not as above 10

10. Corolla lobes with deep purple spot on either sides at the base C. anantii

10. Corolla lobes without deep purple spot on either sides at the base. 11

11. Flowers $c .7 .5 \mathrm{~cm}$ long; corolla lobes attenuate. C. attenuata

11. Flowers c. $3.5 \mathrm{~cm}$ long; corolla lobes obovate... C. anjanerica

12. Corolla lobes fimbriate at the base; cage conical C. fimbriifera

12. Corolla lobes glabrous at the base; cage globular C. noorjahaniae

Ceropegia anantii S.R. Yadav, Sardesai \& S.P. Gaikwad, J. Bombay Nat. Hist. Soc. 101(1): 141. 2004; Karthik. et al., Fl. Pl. India 1: 160. 2009; Kambale \& S.R. Yadav, Asklepios 115: 29. 2013. Type: INDIA, Maharashtra, Sindhudurg district, Salva hill, 15.09. 1998, S.R. Yadav 995A (holo CAL!; iso K!, BSI!, BLAT, SUK!)

Fig. 48

Vernacular name: Ghayal (Marathi).

Perennial erect herbs. Rootstock tuberous; tubers $1.5-3 \mathrm{~cm}$ in diam., depressed globose; roots fibrous. Stem $c .30 \mathrm{~cm}$ high, terete, usually one from a single tuber, unbranched, sparingly hairy, green or dark purple. Lamina 4-9 ×0.4-1 cm, linear, acute at apex, narrow at base, scabrous above with bulbous based hairs, ciliolate along margins, glabrous beneath except for midrib. Flowers solitary, extra-axillary; peduncles 4-7 mm long, terete, puberulous; bracts 2.5-4 mm long, linear-subulate, ciliolate along margins at upper-half, glabrous otherwise; pedicels 0.5-1 cm long, terete, puberulous. Sepals 5.5-8 $\mathrm{mm}$ long, linear-subulate or subulate hairy along midnerve. Corolla $4.6-6.5 \mathrm{~cm}$ long, greenish- yellow; tube $2.2-4 \mathrm{~cm}$ long, abruptly dilated at base, glabrous, greenish outside, dilated part yellow with dark purple striation up to the throat of tube; lobes $1.5-3.5 \mathrm{~cm}$ long, connate at tip and forming long conical beak, occasionally coiled, pubescent within, greenish white, each lobe with dark purple spot on either side at the base of corolla lobes (spot rarely absent). Corona stipitate, bi-seriate; outer of 5-bifid lobes, cupular, 2-3 mm long, ciliate along margins and within, yellow blotched with purple at margins; inner of 5 erect lobes, slightly hooked at tip or connivent, yellow, purple at base, $2.5-3.2 \mathrm{~mm}$ long. Pollinarium c. $0.4 \times 0.3 \mathrm{~mm}$. Follicles $6-8.5 \mathrm{~cm}$ long, usually in pairs, rarely single, erect, tapering to a fine point. Seeds c. $0.4 \times 0.15 \mathrm{~cm}$, ovate-oblong, brown, comose; coma silky white.

Flowering \& fruiting: August-September.

Chromosome number: $2 n=22$ (Gosavi et al., 2012).

Habitat: Grows in low elevation (65-400 m) lateritic plateaus amidst bushes, in association with Tylophora fasciculata Buch.-Ham. ex Wight and Wrightia pubescens R.Br.

Distribution: Endemic to northern Western Ghats of Maharashtra, India.

Specimens examined: INDIA, Maharashtra, Sindhudurg district, Fonda, 16.08.2010, S.S. Kambale SUK 2560; Ibid., 20.08.2010, S.S. Kambale SUK 2562; Ibid., 07.07.2011, S.S. Kambale SUK 2578; Ibid., 05.08.2012, S.S. Kambale SSK 6; Ibid., 04.08.2013, S.S. Kambale SSK 59; 24.07.2014, S.S. Kambale SSK 252; Ibid., 15.08.2014, S.S. Kambale SSK 270; Ibid., 21.08.2014, S.S. Kambale SSK 281; Ibid., 24.08.2014, S.B. Chaugule \& S.S. Kambale SSK 291; Salva hills, s.d,. S.R. Yadav s.n. (SUK!).

Conservation status: So far this species could be collected only from the type locality where the area of occupancy is below10 sq. $\mathrm{km}$ and the plants are facing severe threat due to habitat loss. Therefore it is assessed as Critically Endangered [CR: B2 a, b (iii, v)].

Notes: This species is similar to C. attenuata but can be easily identified by its purple spots on either sides of the base of corolla lobes and tube abruptly dilated at the base. However, very rarely, the flowers without purple spots and a gradually dilated corolla tube are observed in the field.

Ceropegia anjanerica Malpure, M.Y. Kamble \& 

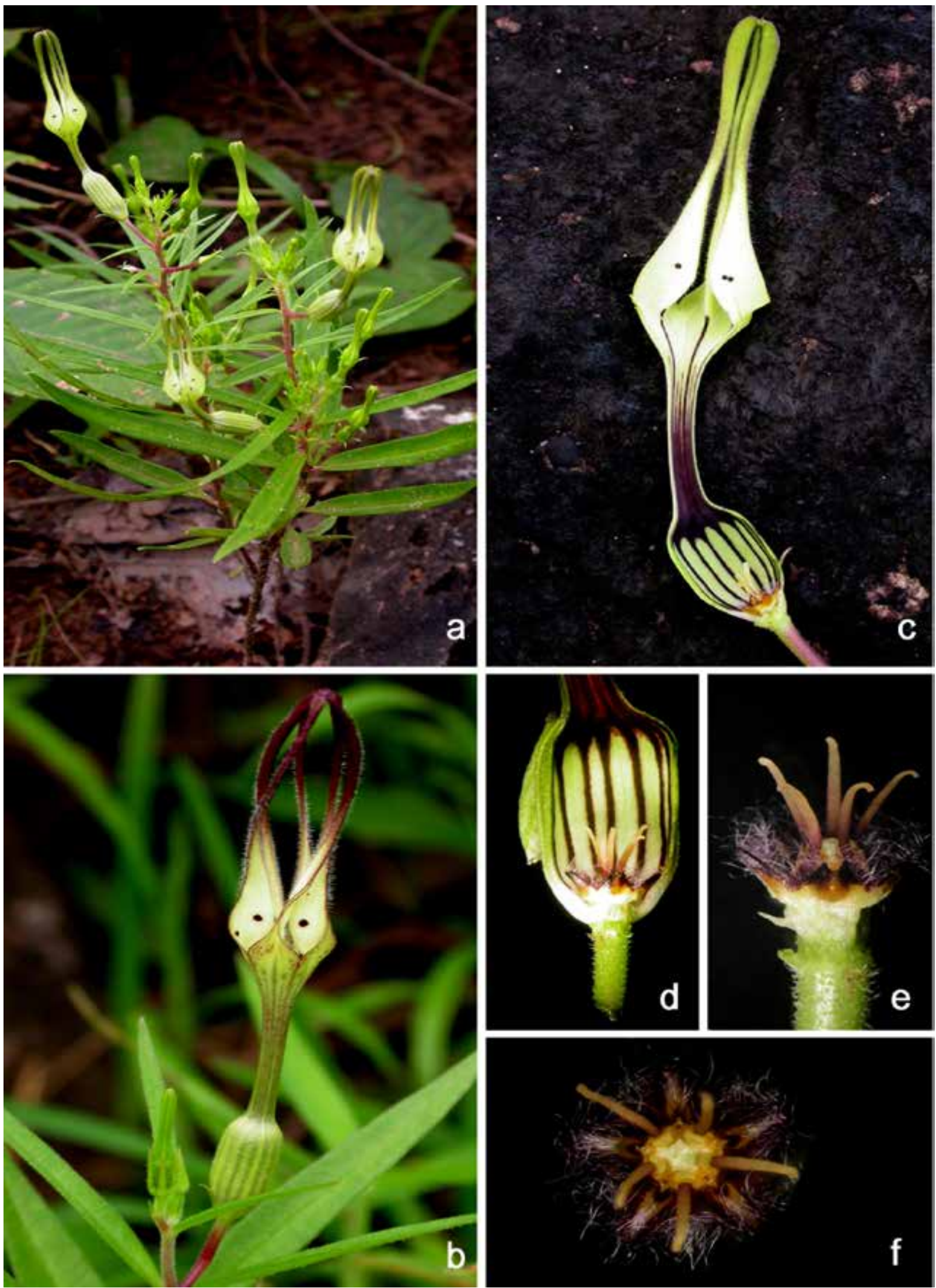

b

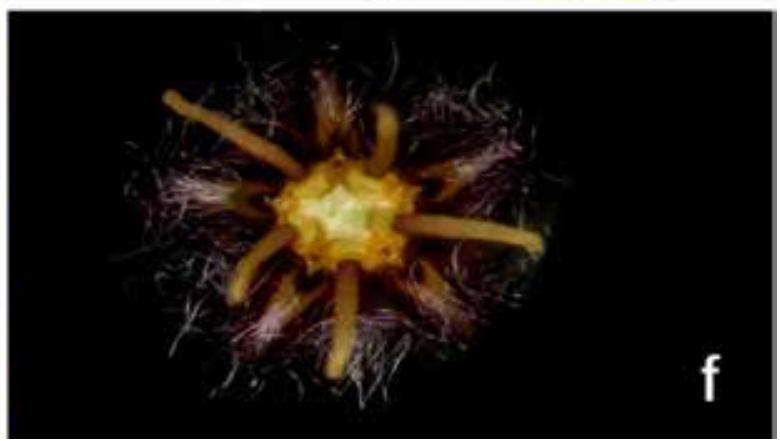

Fig. 48. Ceropegia anantii S.R. Yadav, Sardesai \& S.P. Gaikwad: a. Habit; b. Flower; c. L.S of flower; d. Close-up of dilated portion; e. Coronalateral view; f. Corona-top view. 
S.R. Yadav, Curr. Sci. 91(9): 1141. 2006; Karthik. et al., Fl. Pl. India 1: 160. 2009; Kambale \& S.R. Yadav, Asklepios 115: 29. 2013. Type: INDIA, Maharashtra, Nashik district, Anjaneri hill, 10.09.2005, N.V. Malpure Malpure 1 (holo CAL!; iso K!, BSI!, BLAT!, SUK!).

Fig. 49

Perennial erect herbs. Rootstock tuberous; tubers 2-5 cm in diam., depressed globose to discoid, roots fibrous. Stem terete, $10-20 \mathrm{~cm}$ high, 1-2 $\mathrm{mm}$ in diam., usually unbranched, scabrid. Leaves sub-sessile to petiolate; lamina elliptic-narrowly elliptic, 1.3-3.7 × 0.3-1.1 cm, mucronate at apex, tapering at base, scabrous above, along midrib and margins, glabrous otherwise; petioles 2-6 mm long. Flowers solitary, extra-axillary; peduncles 1-3 mm long, scabrid; bracts c. $1.6 \mathrm{~mm}$ long, subulate; pedicels c. $4 \mathrm{~mm}$ long, scabrous. Sepals c. $3 \times 0.15 \mathrm{~mm}$, subulate, hairy along midnerve, glabrous otherwise. Corolla $1.8-3.5 \mathrm{~cm}$ long; tube $0.8-1.5 \mathrm{~cm}$ long, gradually dilated at base, funnelshaped towards throat, slightly curved, glabrous within, greenish-grey, striated with deep purple lines within, white otherwise; lobes $1-1.8 \mathrm{~cm}$ long, obovate, attenuate, finely pubescent throughout, connate at tip forming an obovate cage, lobes reflexed on their back, greenish-yellow. Corona biseriate, stipitate; outer of 5-bifid lobes, c. $2 \times 2 \mathrm{~mm}$, ciliate within and along margins, yellow; inner of 5 erect linear lobes, $2 \mathrm{~mm}$ long, alternating with outer corona. Pollinarium $c .0 .6 \times 0.5 \mathrm{~mm}$. Follicles usually in pairs, $c .7 \mathrm{~cm}$ long, straight, tapering to a fine point, erect. Seeds c. $5 \times 2 \mathrm{~mm}$, ovate, oblong, comose; coma 1-1.6 cm long, silky white.

Flowering \& fruiting: September-October.

Chromosome number: $2 n=22$ (Gosavi et al., 2012)

Habitat: Grows at an elevation of $1296 \mathrm{~m}$ in well-drained soil, in association with Curcuma neilgherrensis Wight, Senecio bombayensis N.P. Balakr., Strobilanthes callosa Nees and Swertia minor Knobl.

Distribution: Endemic to Maharashtra, India.

Specimens examined: INDIA, Maharashtra, Nasik district, Anjaneri hill, 31.09.2010, S.S. Kambale SUK 2564; Ibid., 18.10.2011, S.S. Kambale SSK 2962; Ibid., 06.10.2012, S.S. Kambale SSK 23; Ibid., 12.08.2013, S.S. Kambale SSK 67; Ibid., 23.10.2013, S.S. Kambale SSK 97 (SUK!).
Conservation status: Though it is newly described species, it has not been located elsewhere in Western Ghats after extensive and intensive field survey. The area of occupancy is below10 sq. $\mathrm{km}$. Therefore it is assessed as Critically Endangered [CR: B2 a, b (i, ii, iii)].

Notes: Similar to C.attenuata but easily distinguished by its shorter corolla, obovate, flat corolla lobes, forming an obovate head. Ceropegia anjanerica grows in lateritic soil of high elevation mountains while the latter is restricted to the low elevation lateritic plateaus.

Ceropegia arnottiana Wight, Contr. Bot. India 32. 1834; Decne. in Prodr. 8: 644. 1844; Hook.f., Fl. Brit. India 4: 74. 1883; P.C. Kanjilal et al., Fl. Assam 3: 309. 1939; H. Huber, Mem. Soc. Brot. 12: 52. 1957; Ansari, Fasc. Fl. India 16: 9. 1984; M.P. Nayar \& Sastry, Red Data Book Indian Pl. 2: 40. 1988; Karthik. et al., Fl. Pl. India 1: 160. 2009. Lectotype (designated by Huber, 1957): MYANMAR, lower Burma, Prome hills, s.d., N. Wallich Wall. Asclep. n. 3 (K000857820 image!; isolectotype E00179560 image!).

Perennial twining herbs. Rootstock a globose tuber. Stem 50-70 cm high, slender, glabrous. Lamina 4.5-10.5 × 0.5-2.6 cm, narrowly lanceolateelliptic, base narrowed, apex acute-mucronulate, sparsely hairy above and ciliate along margins, glabrous beneath; petioles $0.3-1.2 \mathrm{~cm}$ long, slender, glabrous. Cymes 4-6-flowered, extraaxillary; peduncles $1.5-2.3 \mathrm{~cm}$ long, glabroussparsely hairy; bracts c. $4 \mathrm{~mm}$ long, linear, glabrous; pedicels 3-5 mm long, terete, glabrous. Sepals 4-7 $\mathrm{mm}$ long, subulate, glabrous. Corolla $2.7-5.4 \mathrm{~cm}$ long; tube 1.2-2.2 cm long, inflated in lower 1/3$1 / 4$ part, narrow at middle, funnel-shaped at apex, glabrous within; lobes $1.5-3.2 \mathrm{~cm}$ long, linear, connate at tip, hairy along margins. Corona biseriate; outer of 5-bifid, sparsely-ciliate lobes; inner of 5 erect linear lobes. Follicles $c .3 .5 \mathrm{~cm}$ long.

Flowering: September.

Distribution: Northeast India, Burma and Thailand. Conservation status: Nayar and Sastry (1987) assessed it as Endangered or possibly extinct. In present study, authors could not collect this plant and no specimen was found in any of the Indian herbaria. This species is treated here based on Huber (1957) 

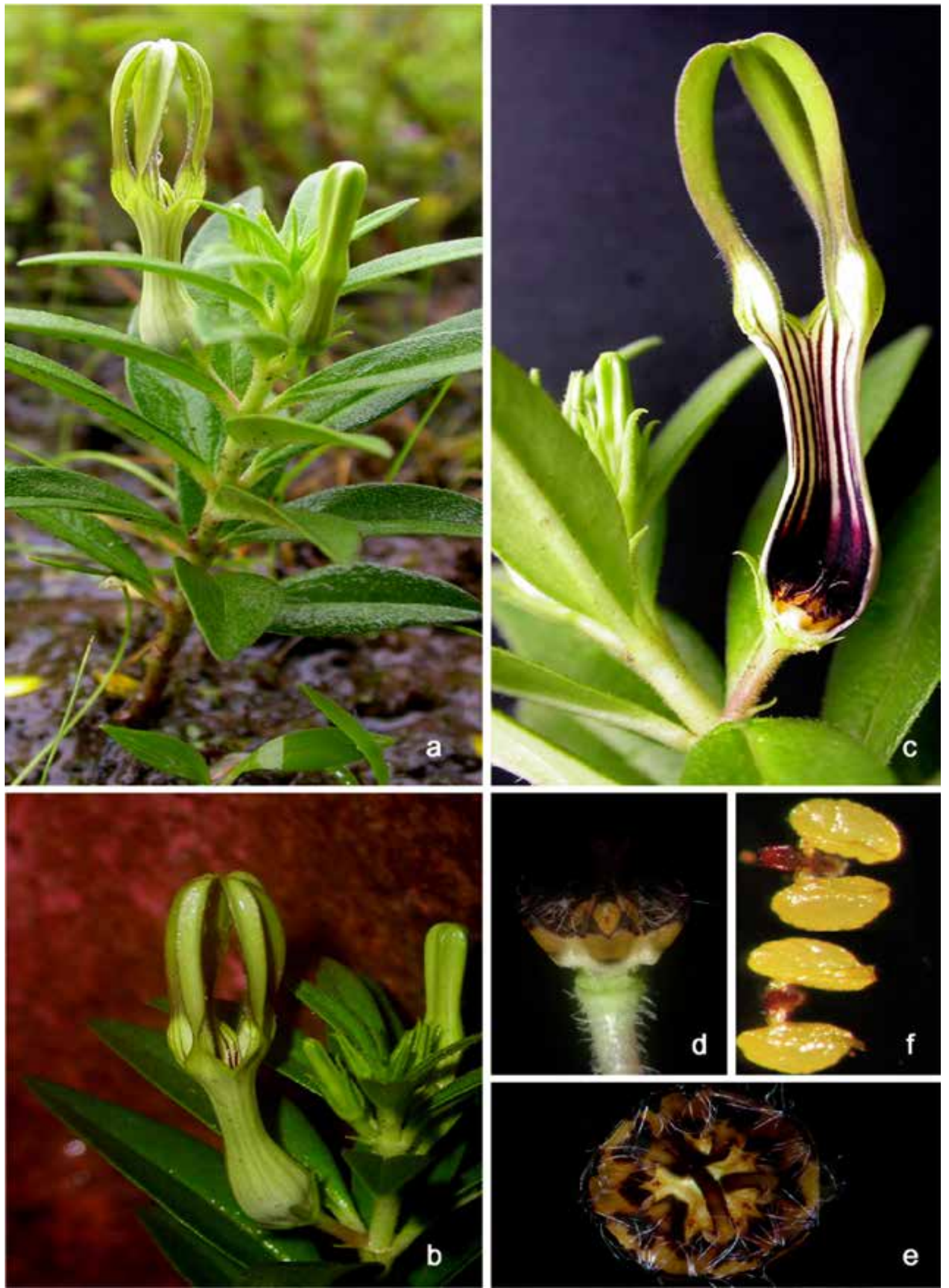

Fig. 49. Ceropegia anjanerica Malpure, M.Y. Kamble \& S.R. Yadav: a. Habit; b. Flower; c. Flower L.S.; d. Corona-lateral view; e. Corona-top view; f. Pollinarium. 
and Ansari (1984).

Notes: It is similar to C. ensifolia Bedd. but differs by its short peduncles, hairy corolla lobes, sparsely ciliate outer corona and linear inner corona.

Ceropegia attenuata Hook., Icon. Pl. 9: t. 867. 1852; Dalzell \& A. Gibson, Bombay Fl. 154. 1861; Hook.f., Fl. Brit. India 4: 67. 1883; T. Cooke, Fl. Bombay 2: 238. 1905; Blatter \& McCann, J. Bombay Nat. Hist. Soc. 36: 534. 1933; H. Huber, Mem. Soc. Brot. 12: 128. 1957; A.R. Kulk. \& Thite, J. Bombay Nat. Hist. Soc. 74: 600. 1979; Ansari, Fasc. Fl. India 16: 9. 1984; R.S. Rao, Fl. Goa, Diu, Daman, Dadra \& Nagarhaveli 2: 262. 1986; M.P. Nayar \& Sastry, Red Data Book Indian Pl. 1: 49. 1987; S.M. Almeida, Fl. Savantwadi 1: 257. 1989; A.P. Jagtap \& N.P. Singh, Fasc. Fl. India 24: 215. 1999; D.K. Mishra \& N.P. Singh, Endem. Threat. Pl. Maharashtra 130. 2001; A.P. Jagtap \& Das Das, N.P. Singh et al., Fl. Maharashtra, Dicot. 2: 347. 2001; F. Albers \& Meve, Ill. Handb. Succ. Pl. Asclepiadaceae 69. 2002; K.G. Bhat, Fl. Udupi 371. 2003; Karthik. et al., Fl. Pl. India 1: 160. 2009; Kambale \& S.R. Yadav, Asklepios 115: 29. 2013. Type: INDIA, Maharashtra, Bombay, Ghats near Vigorna, s.d., N.A. Dalzell s.n. (K000357705 image!)

C. angustifolia Dalzell, Hooker's J. Bot. Kew Gard. Misc. 2: 259. 1850, non Wight, 1834; Dalzell \& A. Gibson, Bombay Fl. 154. 1861. nom. illeg. C. attenuata Hook. var. mookambikae Diwakar \& R. K. Singh, Indian J. Forest. 34(2): 209. 2011. Type: INDIA, Karnataka, Udupi district, Mookambika Wildlife Sanctuary, Manmanhara plateau, 26.08.2007, P.G. Diwakar \& R. K. Singh 191852A (holo CAL!).

Fig. 50

Vernacular names: Tilori, Tilor, Kaper halda (Marathi).

Perennial erect herbs. Rootstock tuberous; tubers 2-4 cm diam., globose, depressed; roots fibrous. Stem terete, usually single from each tuber, unbranched, rarely branched, pubescent, purple below, green above, c. $30 \mathrm{~cm}$ tall, $0.3 \mathrm{~cm}$ diam. Leaves sub-sessile; lamina $3.2-9.0 \times 0.2-1.2 \mathrm{~cm}$, linear-lanceolate, falcate, acute at apex, narrowed at base, ciliolate along margins and nerve beneath, glabrous otherwise. Flowers solitary, extra-axillary or axillary; peduncles $0.4-0.45 \mathrm{~cm}$ long, terete, pubescent; bracts c. $2.5 \times 0.15 \mathrm{~mm}$, terete; pedicels $0.7-0.9 \mathrm{~cm}$ long, terete, pubescent. Sepals $0.4-0.5$ cm long, hairy along mid nerve. Corolla 5-7.2 cm long; tube $1.8-3.5 \mathrm{~cm}$ long, slightly curved, glaucous, gradually dilated at base, narrow at middle, funnel-shaped above, glabrous, (with 5 angular projections or distinct lines, sometime), parrot green to deep purple, deep purple within at dilated part with dark purple striations up to mouth of corolla tube; lobes $c .3 .7 \mathrm{~cm}$ long linear attenuate, triangular at base, connate at tip forming conical-ovate cage, twisted in bud and at maturity lobes tend to coil, reflexed, pubescent throughout within and along margins, throughout greenpurple (variable in colour). Corona bi-seriate, stipitate; outer of 5-bifid lobes, cupular, c. $2.5 \times 3.5$ $\mathrm{mm}$, ciliate along margins and within; inner erect, divergent at maturity, spathulate, $2.5 \mathrm{~mm}$ long, alternate with outer corona, yellow. Pollinarium $c$. $0.4 \times 0.6 \mathrm{~mm}$. Follicles $6-8.8 \mathrm{~cm}$ long, glabrous, tapering towards apex, usually unequal. Seeds $c$. $3.7 \times 2.2 \mathrm{~mm}$, brown, ovoid-oblong, marginate, comose; coma 1-1.5 cm long, silky white.

Flowering \& fruiting: July-October.

Chromosome number: $2 n=22$ (Raghavan \& Ansari, 1975).

Habitat: Grows in rock crevices and soil pockets on plateaus of Western Ghats. Its typical associates include Flemingia nilgheriensis (Baker) T. Cooke, Glyphochloa forficulata (C.E.C. Fisch.) Clayton, Wrightia tinctoria R.Br., etc.

Distribution: Endemic to Peninsular India. Reported from Arunachal Pradesh by Giri et al. (2008) but without any herbarium data. Since, C. attenuata is restricted to low elevation lateritic plateaus in Konkan, authors assume that whatever Giri (l.c.) observed might be C. poluniniana Bruyns from Nepal. Similarly its report from Rajasthan by Shetty (1991) is also a misidentification of $C$. bulbosa.

Specimens examined: INDIA, Goa, Loliem Plateau, 20.08.2012, S.S. Kambale SSK 13; Verna village plateau, 19.08.1963, K.C. Kanodia 89351 (BSI!); Ibid., 24.07.2014, S.S. Kambale SSK 246 (SUK!). Karnataka, way Honnawar to Karwar, 07.04.2012, S.S. Kambale 3; Karwar, 21.08.2014, S.S. Kambale SSK 280; Ibid., 10.09.1987, W.A. Talbot s.n. (BSI!); way Karwar to Honnawar, 20.08.2012, S.S. Kambale SSK 12 (SUK!); Udupi district, Bhatkal, 19.09.2014, S.S. Kambale SSK 320 

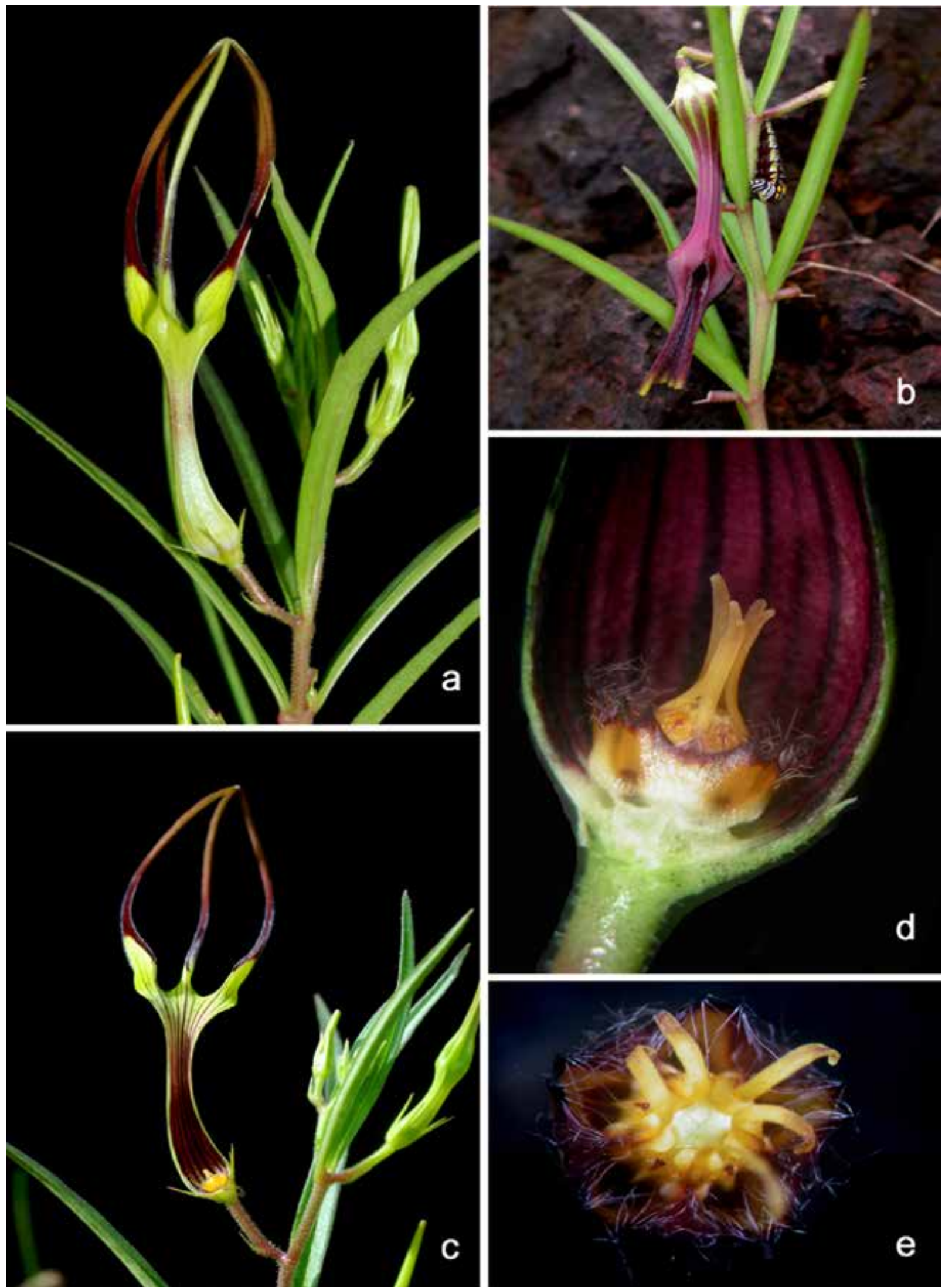

Fig. 50. Ceropegia attenuata Hook.: a. Habit; b. Butterfly larva feeding on the leaves and flowers; c. Flower L.S.; d. Corona-lateral view; e. Corona-top view. 
(SUK!); Durga Parameshwari Temple, 19.08.2012, S.S. Kambale SSK 11; Mookambika Wildlife Sanctuary, Mammanhara Plateau, 19.07.2009, P.G. Diwakar \& R.K. Singh 185109 (BSI!); Uttara Kannada district, Castle rock, 25.10.1902, G.A. Gammie 15617. Maharashtra, Concan, s.d., Stocks \& Law s.n. (CAL!); Nasik district, Near Kasara, 23.09.1968, Karl Vore 115319 (BSI!); Pune district, Khandala, 03.07.1961, M.Y. Ansari 32781 (BSI!); Raipur district, Bhatgaon, 09.09.2013, S.S. Kambale SSK 86; Jaitapur, 29.07.2014, A.V. Mohite \& S.S. Kambale SSK 253; Ibid., 10.08.2014, A.R. Gholave E S.R. Yadav SSK 263; Ibid., 09.10.2014, S.S. Kambale \& S.R. Yadav SSK 289; Sanjay Gandhi National Park, Borivali, Mumbai, 17.07.2014, S.S. Kambale SSK 243; 02.09.2014, S.S. Kambale SSK 300 (SUK!); Ratnagiri district, Bhate, 22.09.1990, S.R. Yadav 5915; Ratnagiri, 05.09.1999, V.T. Patil E R.S. Gaikwad 199; Ibid., 02.07.2003, M.Y. Kamble 2123; Ibid., 05.09.1999, N.R. Patil 406; 16.07.2011, S.S. Kambale SUK 2562; Dapoli, 09.09.2013, S.S. Kambale SSK 87 (SUK!) Ibid., Pangari, 09.09.2013, S.S. Kambale SSK 85; Ibid., 16.07.2011, S.S. Kambale SUK 2577; Pawas, 01.08.2011, S.S. Kambale \& S.R. Yadav SUK 2563; Chiplun, Pochri, 19.08.2011, S.S. Kambale SUK 2594; Mumbai, Borivali, Canary Caves, 15.09.2011, S.S. Kambale SUK 2599; 04.08.2013, S.S. Kambale SSK 60; Ibid., 07.09.2013, S.S. Kambale SSK 82; Guhagar, 09.09.2013, S.S. Kambale SSK 84 (SUK !); Thane district, Mumbra hill top, Thana range, 09.09.1968, Karl Vore 116484 (BSI!).

Conservation status: Nayar and Sastry (1987) assessed it as Rare while Mishra and Singh (2001) as Vulnerable. Plants of the species are locally abundant in its area of occurrence. However, modification of habitats for mango cultivation, construction of resorts and consumption of Ceropegia tubers by local inhabitants are the major threats. In the present study it is assessed as Vulnerable [VN: B2 b (iii, iv, v), c (i, iii, v)].

Notes: Allied to C. spiralis Wight but differs from it in having linear corolla lobes, gradually dilated corolla tube and in habitat preferences (low elevation lateritic plateaus). This taxon is also close to $C$. fimbriifera but the latter can be distinguished by its shorter (than tube) and fimbriate corolla lobes. This species is exceptionally variable in the length of the flower, shape and colour of corolla lobe cage.

Ceropegia concanensis Kambale, Chandore \& S.R. Yadav, Kew Bull. 67(4): 843. 2012; Kambale \& S.R. Yadav, Asklepios 115: 29. 2013. Type: INDIA, Maharashtra, Sindhudurg, Vengurla, 28.08.2011, S.S. Kambale 3130 (holo CAL!; iso K001070967 image!, SUK!).

Fig. 51

Perennial erect herbs. Rootstock tuberous; tubers 2-5 cm in diam., globose, ovoid or depressed; roots fibrous. Stem solitary, terete, unbranched, $c$. $30 \mathrm{~cm}$ high, 1-2 mm diam., more or less pubescent when young, glabrous when mature, green or dark purple. Leaves sub-sessile; lamina 3.2-6.4 $\times$ 0.8-1.4 cm, linear, acute, narrowed at base, midrib prominent, puberulous on the upper surface and glabrous beneath, ciliolate at margins. Flowers solitary, extra-axillary; peduncles 3-5 mm long, slender, pubescent; bracts solitary, subulate, $2-3 \times$ $0.2-0.4 \mathrm{~mm}$, hairy along the margins; pedicels 5-6 $\times 1-1.5 \mathrm{~mm}$, slender, pubescent. Sepals 4-7 $\times 0.3-$ $0.6 \mathrm{~mm}$, acute, hairy along the midnerve. Corolla 5-lobed, 4-5.4 cm long, usually slightly curved, purple; tube $3-3.5 \mathrm{~cm}$ long, abruptly dilated at base, cylindrical at middle, funnel-shaped at throat, glabrous, purple outside, pale yellowish-green at dilated base, striated with dark purple lines inside in upper-half and dark purple with light windows in lower half; lobes $1.2-1.6 \mathrm{~cm}$ long, narrowly linear, reflexed on their back, connate at the tips forming ovoid cage, pubescent along margins, yellowish-green to reddish-yellow, blotched with dark purple spots at the base. Corona bi-seriate, 4-5 mm long; outer cupular, entire, deeply bifid at the time of shading, segments acute, sparsely ciliate along the margins; inner of 5 linear lobes, erectodivergent at apex, purple at lower half and yellow at upper-half. Pollinarium $c .0 .3 \times 0.2 \mathrm{~mm}$. Follicles in pairs, c. $8 \times 0.3 \mathrm{~cm}$, straight, tapering at both ends, glabrous. Seeds c. $4 \times 2 \mathrm{~mm}$, brown, ovateoblong, comose; coma c. $1 \mathrm{~cm}$ long.

Flowering \& fruiting: July-October.

Habitat: Grows on lateritic plateaus at an elevation ranging from 50-750 m. Typical associates are Dimeria spp., Eriocaulon spp., Euphorbia antiquorum L., Glyphochloa spp., Hedyotis pumila L.f., Impatiens tomentosa Heyne, Leucas aspera (Willd.) Link, Rhamphicarpa longiflora Wight ex Benth. and Striga gesnerioides (Willd.) Vatke. 

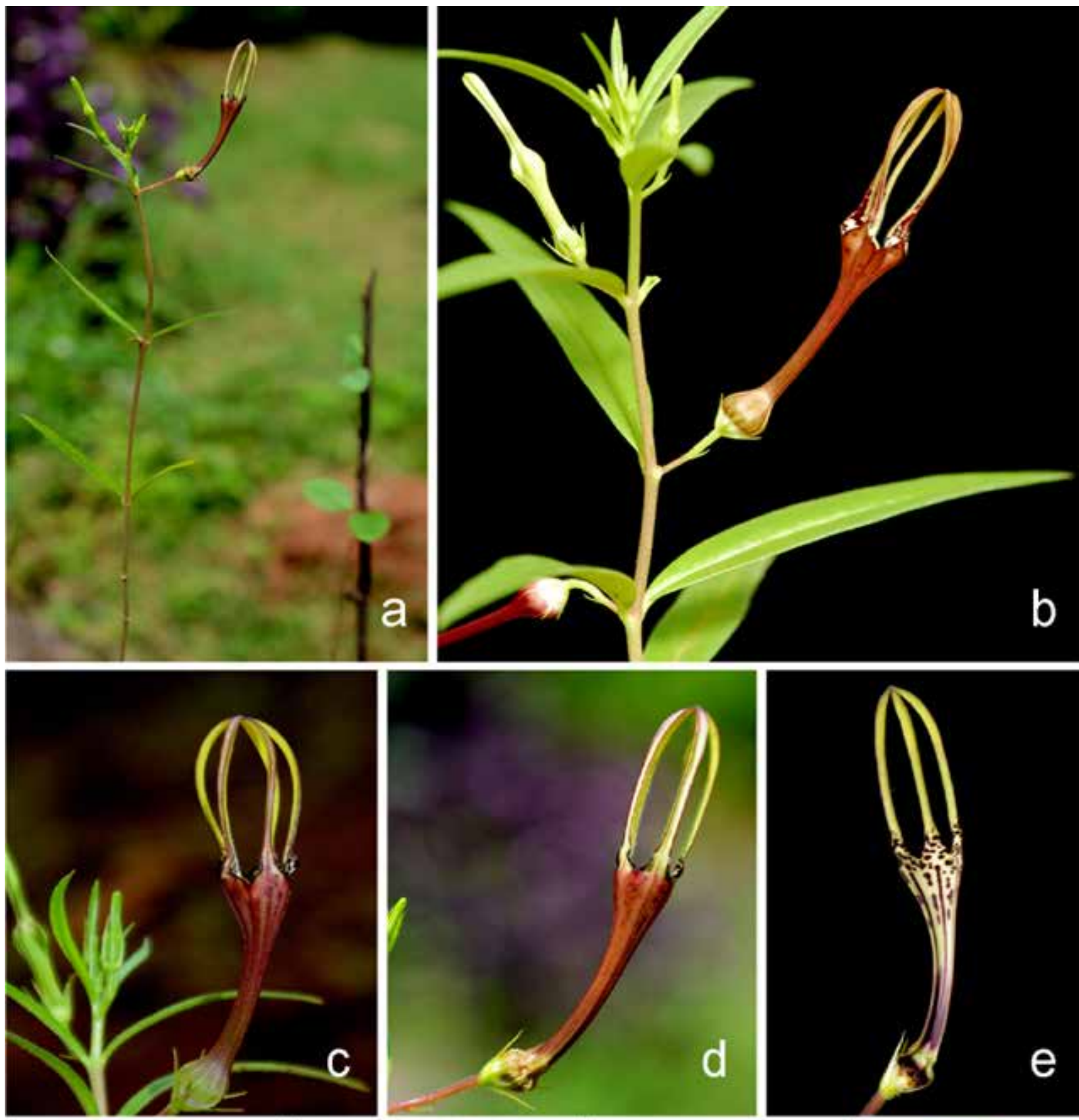

e
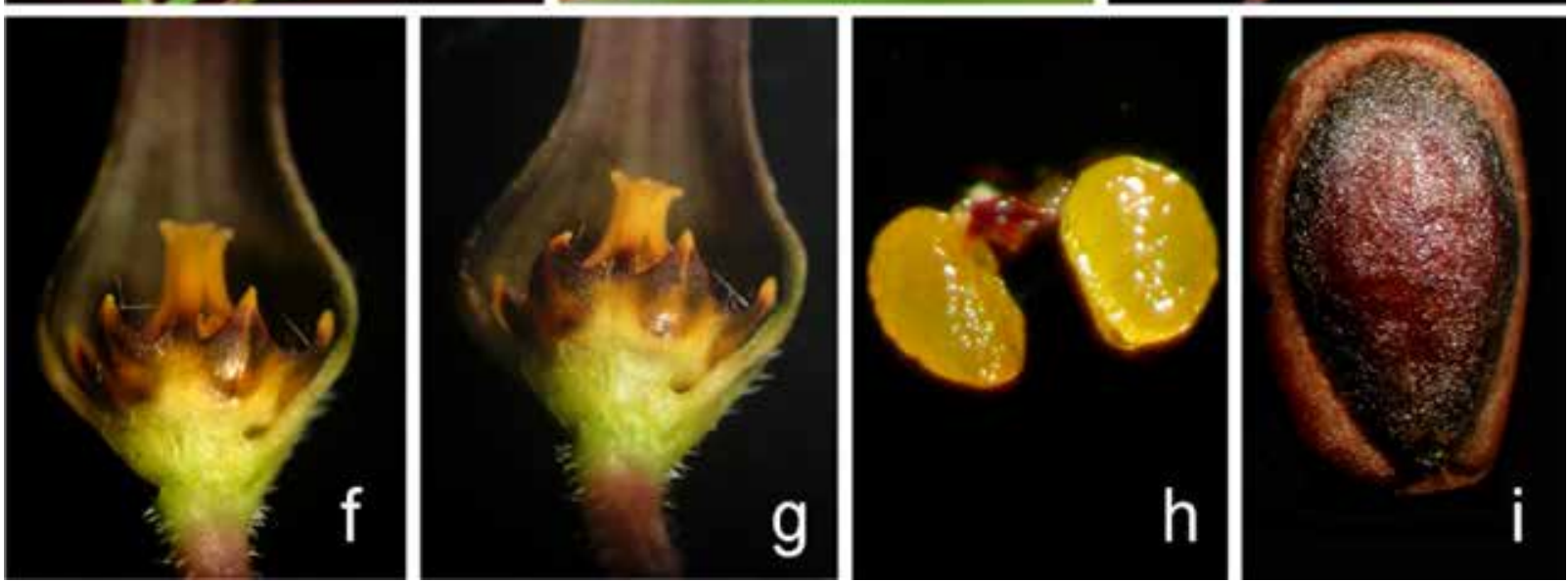

Fig. 51. Ceropegia concanensis Kambale, Chandore \& S.R. Yadav: a \& b. Habit; c \& d. Flower; e. Flower L.S.; f \& g. Corona; h. Pollinarium; i. Seed. 
Distribution: Endemic to Maharashtra (India).

Specimens examined: INDIA, Maharashtra, Kolhapur district, Tillari, 08.08.2010, S.S. Kambale 3131; 15.08.2010, A.N. Chandore 1531 (SUK!); Sindhudurg district, Vengurla, 28.08.2011, S.S. Kambale 3130; Tillari Ghat, 26.09.2014, S.S. Kambale, K. Das \& A.R. Gholvae SSK 321 (SUK!).

Conservation status: Ceropegia concanensis is assessed as Critically Endangered [CR: B1 a, b (i, ii, iii)]. Exploitation of edible tubers by local people and conversion of lateritic plateaus for mango cultivation are the major threats to the species (Kambale et al., 2012).

Notes: Ceropegia concanensis is similar to C. attenuata but can be easily identified by its corolla lobes shorter than tube ( $v s$. lobes equal to tube), abruptly dilated corolla tube ( $v$ s. gradually dilated tube) and sparsely hairy outer corona ( $v s$. densely hairy corona).

Ceropegia fimbriifera Bedd., Madras J. Lit. Sci. Ser. 3(1): 53. 1864 et Icon. Pl. Ind. Or. 1: 38, t. 172. 1874; Hook.f., Fl. Brit. India 4: 66. 1883; Gamble, Fl. Madras 2: 856. 1921; H. Huber, Mem. Soc. Brot. 12: 127. 1957; B.D. Sharma et al. Fl. Karnataka, Analysis 166. 1984; Ansari, Fasc. Fl. India 16: 16. 1984; M.P. Nayar \& Sastry, Red Data Book Indian Pl. 1: 57. 1987; S.R. Sriniv. in Henry et al., Fl. Tamil Nadu Ind., Ser I, Analysis 2: 83. 1987; F. Albers \& Meve, Ill. Handb. Succ. Pl. Asclepiadaceae 79. 2002; Nayar et al., Fl. Pl. Kerala 85. 2006; Bruyns, Rheedea 7(2): 113. 1997; A.P. Jagtap \& N.P. Singh, Fasc. Fl. India 24: 223. 1999; Karthik. et al., Fl. Pl. India 1: 161. 2009; Kambale \& S.R. Yadav, Asklepios 115: 31. 2013. Lectotype (designated by Huber, 1957): INDIA, Tamil Nadu, Annamalay hills, s.d., R.H. Beddome s.n. (BM001014217 image!)

Fig. 52

Perennial erect (twining under cultivation) herbs. Rootstock tuberous; tubers $1-2.6 \mathrm{~cm}$ in diam., sub-globose; roots fibrous. Stem usually solitary, rarely branched, terete, c. 15-30 cm high (c. $2 \mathrm{~m}$ under cultivation), 1-2 $\mathrm{mm}$ in diam., pubescent when young, glabrous when mature, green or dark purple, internodes $1.2-13 \mathrm{~cm}(20-26 \mathrm{~cm}$ under cultivation). Lamina $2.8-12.5 \times 0.6-1.7 \mathrm{~cm}$, elliptic-lanceolate, acute at apex, narrowed at base, pubescent along margins and midnerve beneath, glabrous otherwise; petioles $0.2-1.5 \mathrm{~cm}$ long, channeled above, hairy along margins, glabrous otherwise. Cymes 2-3-flowered, extra-axillary; peduncles $0.2-2.8 \mathrm{~cm}$ long, slender, pubescent; bracts solitary, linear, c. $3 \mathrm{~mm}$ long, pubescent; pedicels 1-1.2 cm, slender, glabrous. Sepals c. 5.5 mm long, linear, glabrous, minutely hairy at the tip. Corolla c. $3.8 \mathrm{~cm}$ long, usually slightly curved, rarely straight; tube $1.5-2 \mathrm{~cm}$ long, gradually dilated at base, glabrous, usually mottled with purple strips at mouth, rarely mottled throughout, deep purple within, striated with purple lines in upper-half; lobes $0.6-1.8 \mathrm{~cm}$ long, narrowly linear, reflexed on their back, connate at the tips to form ovoid cage, glabrous otherwise, green, purple and fimbriate at the base; fimbriae $2.5-3 \mathrm{~mm}$ long, glandular, purple, clavate. Corona bi-seriate; outer of 5 deeply bifid lobes, saucer to bowl-shaped, c. $2 \times 1.5 \mathrm{~mm}$, deep purple along margins, hairy along margins and within; inner of 5 linear to sub-spathulate erect lobes, c. $2 \mathrm{~mm}$ long, glabrous, pinkish purple. Pollinarium erect, c. $0.3 \times 0.2 \mathrm{~mm}$. Follicles in pairs, $c .8 \mathrm{~cm}$ long, straight, tapering at both ends, glabrous.

Flowering \& fruiting: June-November.

Habitat: Occurs amongst grasses on the hill-sides of dry regions in Karnataka and Tamil Nadu. In Kerala it occurs amidst grasses on rocky hill slopes in evergreen forests.

Distribution: Endemic to South India.

Specimens examined: INDIA, Karnataka, Chamarajanagar district, Biligiriranga hills, 06.1938, E. Barnes 1875 (BSI!); Chikkaballapur district, Nandi Hills, 21.08.1976, N.P. Singh 143185 (BSI!); Ibid., 09.08.2013, S.S. Kambale \& S. Surveswaran SSK 63; Ibid. (grown in garden), 23.11.2013, S.S. Kambale \& S. Surveswaran SSK 203 (SUK!); Dakshina Kannada district, Charmadi, 26.10.1996, K. Ravikumar, G.S. Goraya \& S.R. Ramesh 9731 (FRLH!). Kerala, Palakkad district, Silent Valley National Park, 15.10.2010, A.R. Kulavmode 46386 (TBGT!); Ibid. (grown in garden), 06.10.2012, A.R. Kulavmode \& S.S. Kambale SSK 24; Ibid., 19.09.2014, S.S. Kambale \& A.R. Kulavmode SSK 319 (SUK!). Tamil Nadu, Coimbatore district, Annamalay, 1866, R.H. Beddome s.n:; Kadamparai, Attakutti, 21.07.1978, M.C. Bose 57222; Nilgiris district, Ebbanad, Koil peak \& surrounding, 11.09.1970, G.V. Subbarao 

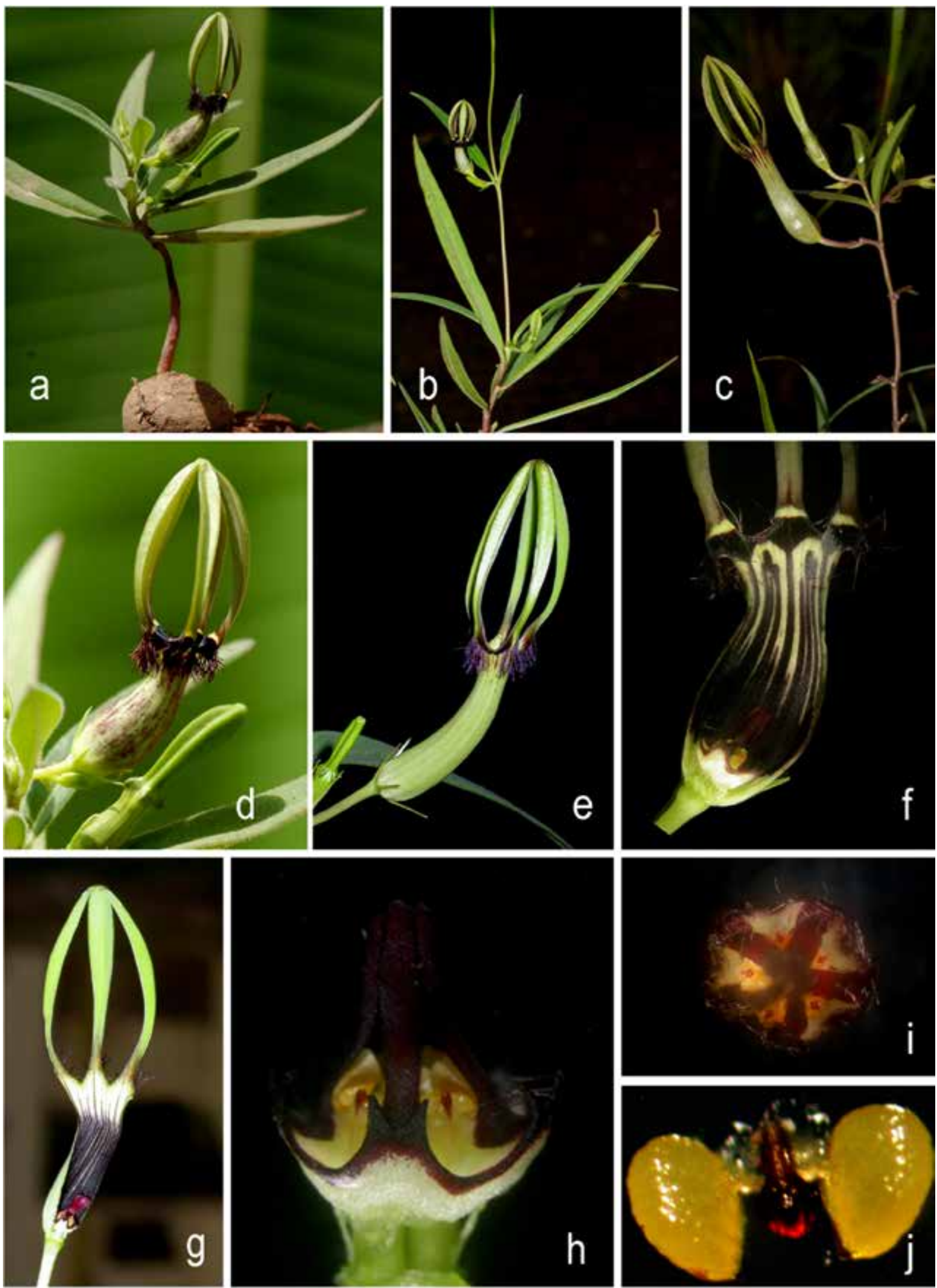

Fig. 52. Ceropegia fimbriifera Bedd.: a \& b. Habit; c. Flower without fimbria; d \& e. Flowers with fimbria; $\mathbf{f}$ \& g. Flower L.S.; h. Corona-lateral view; i. Corona-top view; j. Pollinarium. 
36637 (MH!); Ibid., 21.11.2012, S.S Kambale \& S.R. Yadav SSK 28; Ibid. (grown in garden), 23.10.2013, S.S Kambale \& S.R. Yadav SSK 95 (SUK!).

Conservation status: This plant is poorly represented in Indian herbaria. Nayar and Sastry (1987) assessed it as Vulnerable. Populations are very sparse as very few individuals where located during field surveys and a thorough field observation is required. It is assessed as Data Deficient (DD).

Notes: Ceropegia fimbriifera is allied to C. noorjahaniae but differs in having fimbriate corolla lobes and gradually dilated corolla tube. It is also closer to C. bhatii but can be distinguished by its reflexed, fimbriate corolla lobes and corolla tube mottled with purple at the throat outside and deep purple inside. In C. bhatii flowers are deep green throughout, corolla lobes not reflexed on the back, they are glabrous throughout and corolla tube reddish mottled within. However plants collected from Kerala are without fimbriae or trichomes at the base of corolla lobes.

Ceropegia jainii Ansari \& B.G. Kulk., Bull. Bot. Surv. India 22(1-4): 221. 1982; Ansari, Fasc. Fl. India 16: 18. 1984; M.P. Nayar \& Sastry, Red Data Book Indian Pl. 2: 43. 1988; S.M. Almeida, Fl. Savantwadi 1: 258. 1989; Bachulkar et al., J. Bombay Nat. Hist. Soc. 92 (2): 291. 1995; A.P. Jagtap \& N.P. Singh, Fasc. Fl. India 24: 226. 1999; D.K. Mishra \& N.P. Singh, Endem. Threat. Pl. Maharashtra 135. 2001; A.P. Jagtap \& Das Das in N.P. Singh et al., Fl. Maharashtra, Dicot. 2: 351. 2001; M.R. Almeida, Fl. Maharashtra 3A: 232. 2001; F. Albers \& Meve, Ill. Handb. Succ. Pl. Asclepiadaceae 83. 2002; Karthik. et al., Fl. Pl. India 1: 162. 2009; Kambale \& S.R. Yadav, Asklepios 115: 35. 2013. Type: INDIA, Maharashtra, Sindhudurg district, Amboli Ghat, s.d., Kulkarni 121885A (holo CAL!).

Fig. 53

Perennial erect herbs. Rootstock tuberous; tubers sub-globose, depressed. Stem 5-16.5 cm (under cultivation $55 \mathrm{~cm}$ ), terete, unbranched; internodes 0.3-4.7 cm long, scabrid, pale to dark purple. Lamina $2.7-5 \times 0.4-0.8 \mathrm{~cm}$, lower ones elliptic, upper ones elliptic-linear, scabrid above, ciliolate along margins and beneath along midnerve, dark green above, pale below; midnerve prominent; petioles 1-2 mm long, channeled above, scabrid. Flowers solitary, extra-axillary; peduncles 1-2 mm long, puberulous; bracts small c. $1.2 \times 0.2 \mathrm{~mm}$, hairy at apex, subulate; pedicels 3-4 mm long, pubescent, slender. Sepals c. $4 \times 2.5 \mathrm{~mm}$, subulate, hairy along midnerve, up to the middle. Corolla $1.8-2.7 \mathrm{~cm}$ long; tube c. $1.3-1.5 \mathrm{~cm}$ long, dilated at the base, green at dilated part, glabrous, purple otherwise, slightly curved, with deep purple striations in the dilated part; lobes c. $9 \mathrm{~mm}$ long, linear-oblong, acute and connate at the tip forming globose cage, $8 \mathrm{~mm}$ across, hairy at the base, hairs downwardly directed, glabrous otherwise, greenish-purple. Corona bi-seriate, stipitate; outer of 5-bifid lobes, bowl-shaped, c. $3 \times 3 \mathrm{~mm}$, ciliate along margins and within, yellow blotched with purple; inner of 5 erect lobes, 1.5-2 mm long, divergent, yellowishpurple. Pollinarium c. $0.25 \times 0.25 \mathrm{~mm}$. Follicles tapering towards apex, $5.5-7.5 \mathrm{~cm}$, glabrous, reddish-purple-green. Seeds 3-5 × 1.2-1.5 mm, ovate, oblong, comose; coma 1-1.4 cm long.

Flowering \& fruiting: August-September.

Chromosome number: $2 n=22$ (Gosavi et al., 2012).

Habitat: Grows on high and low elevation lateritic plateaus in Western Ghats in association with Glyphochloa forficulata (C.E.C. Fisch.) Clayton, Jansenella griffithiana (Mull. Hal.) Bor, Lepidagathis cristata Willd. and Rostellularia japonica (Thunb.) J.L. Ellis.

Distribution: Endemic to northern Western Ghats of Maharashtra and Karnataka, India.

Specimens examined: INDIA, Karnataka, Belgaum district, Sada-Kankumbi, 12.08.2012, S.S. Kambale SSK 9; Ibid., 06.09.2013, S.S. Kambale SSK 76 (SUK!). Maharashtra, Ratnagiri district, Chalakewadi, 10.1994, M.P. Bachulkar-Cholekar 5713; Gothane, 10.2003, S.P. Gaikwad SPG 382; Ibid. (grown in garden), 23.09.2012, S.S. Kambale SSK 22; Ibid., 19.09.2014, S.S. Kambale SSK 315 (SUK!); Satara district, Kas, 09.1992, M.P.BachulkarCholekar 5249; Ibid., 06.09.2013, S.S. Kambale SSK 78; Ibid., 16.10.2012, S.S. Kambale SSK 26; Valmiki Plateau, 01.09.2013, S.S. Kambale \& P.R. Lawand SSK 72; Ibid., 20.09.2013, PR. Lawand E S.S. Kambale SSK 92; s.loc., 25.09.1999, S.R. Yadav s.n. (SUK!); Sindhudurg district, Amboli, Moose plateau, 13.08.1971, B.G. Kulkarni 131615A (BSI!); Chaukul plateau, 21.09.2013, S.S. Kambale SUK 92; Ibid., 01.09.2014, S.S. Kambale \& K. Das SSK 299; Ibid., 18.10.2014, S.S. Kambale SSK 327 (SUK!) 

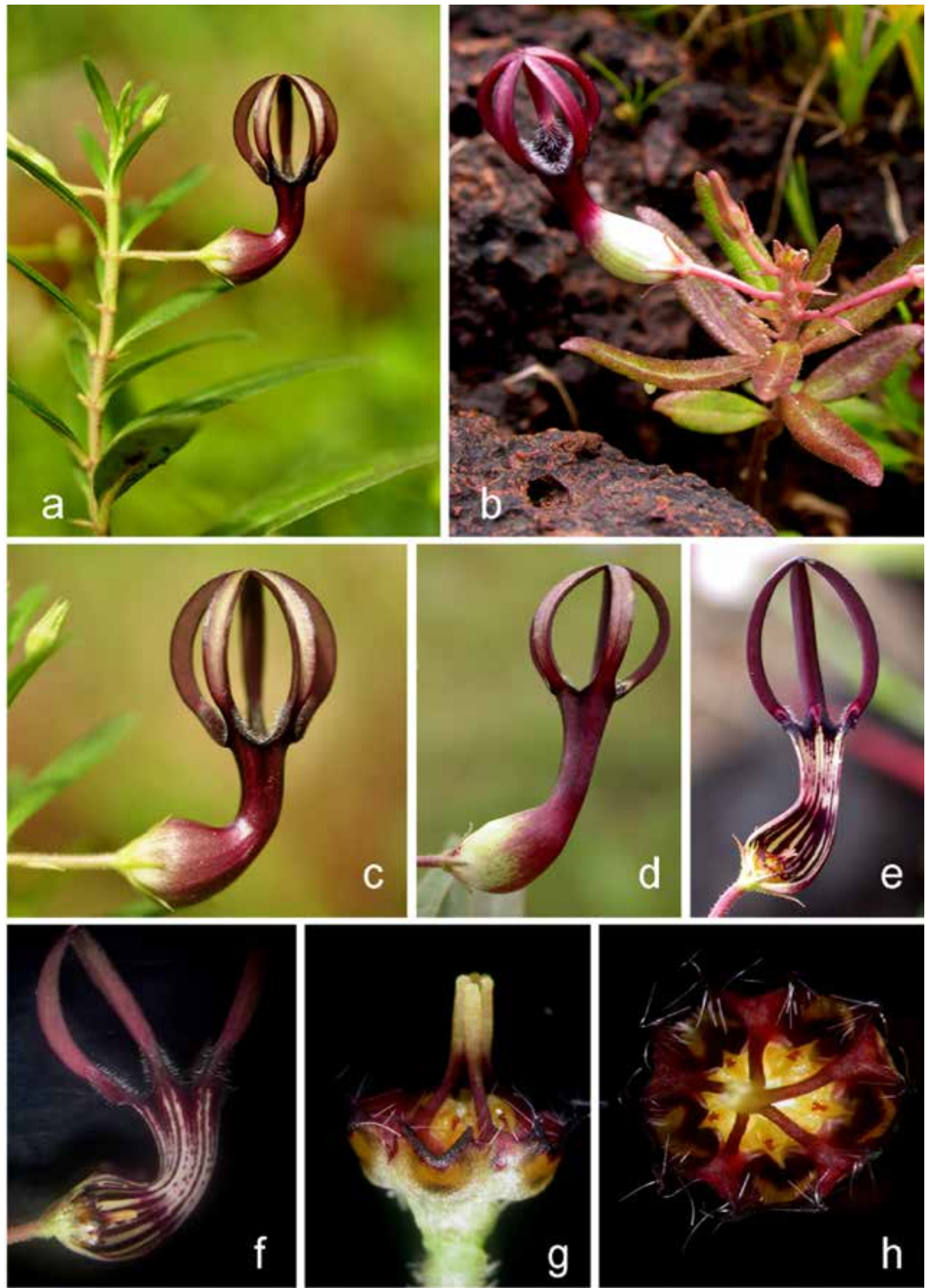

Fig. 53. Ceropegia jainii Ansari \& B.G. Kulk.: a \& b. Habit; c \& d. Flower; e \& f. Flower L.S.; g. Corona-lateral view; h. Corona-top view. 
Conservation status: Nayar and Sastry (1987) assessed it as Rare. Apart from the type locality, this plant has been collected from other localities as well. Inspite of this, it is under threat due to anthropogenic pressures. In the present study, it is assessed as Critically Endangered [CR: B1 a, b (iii, iv, v)].

Notes: Ceropegia jainii is closely related to C. pusilla Wight but differs in having elliptic leaves, reddish flowers and hairy corolla lobes equaling the tube. The plants are comparatively shorter and erect but become a twiner under cultivation with significant elongation of the internodes.

Ceropegia mahabalei Hemadri \& Ansari, Indian Forester 97(2): 105. 1971; Ansari, Fasc. Fl. India 16: 24. 1984; M.P. Nayar \& Sastry, Red Data Book Indian Pl. 2: 49. 1988; A.P. Jagtap \& N.P. Singh, Fasc. Fl. India 24: 232. 1999; D.K. Mishra \& N.P. Singh, Endem. Threat. Pl. Maharashtra 139. 2001; A.P. Jagtap \& Das Das in N.P. Singh et al., Fl. Maharashtra, Dicot. 2: 353. 2001; M.R. Almeida, Fl. Maharashtra 3A: 234. 2001; F. Albers \& Meve, Ill. Handb. Succ. Pl. Asclepiadaceae 89. 2002; Karthik. et al., Fl. Pl. India 1: 162. 2009. Type: INDIA, Maharashtra, Ralegaon hills, west of Junnar, 25.09.1968, K. Hemadri 107266A (holo CAL!; iso BSI!, CAL!, $\mathrm{K}$ !, $\mathrm{L}$ !).

Fig. 54

Vernacular name: Gauti Kharpudi (Marathi).

Perennial erect herbs. Rootstock tuberous; tubers $2-4.5 \mathrm{~cm}$ in diam., sub-globose; roots fibrous. Stem erect, unbranched, 20-24 cm long (40-65 cm long in cultivation), terete, hirsute. Leaves sessile to subsessile; lamina $6.5-9 \times 0.3-0.5 \mathrm{~cm}$, linear to linearlanceolate, acute at apex, narrowed at base, with stiff hairs above, along margins and midrib beneath. Flowers solitary, extra-axillary; peduncles 1-3 mm long, hirsute; bracts 5-6 mm long, , subulate, hairy along midnerve and margins; pedicels 6-9 mm long, hairy. Sepals $1.2-1.4 \mathrm{~cm}$ long, subulate, densely hairy along midnerve, blotched purple along midnerve. Corolla 7.5-9.5 cm long; tube 5.3-6 cm long, slightly curved, broadly dilated at base, cylindrical at middle, funnel-shaped at throat, glabrous within, pale green at dilated part, brownish-red towards throat, green with purple striations within the dilated part and brownish-purple above; lobes $2.2-3.5 \mathrm{~cm}$ long, always shorter than tube, reflexed, linear, elongated above from ovate deltoid base, connate at the tip form a beak, finely pubescent within, dark green with brownish-yellow blotches at the base. Corona bi-seriate, stipitate; outer of 5 shortly bifid lobes, $c$. $3 \times 3 \mathrm{~mm}$, saucer-shaped, sparsely ciliate within, yellow, slightly blotched with brown; inner of 5 erect lobes, c. $3 \mathrm{~mm}$ long, connivent, linear, alternate with outer corona, purple at base, yellow otherwise. Pollinarium c. $0.7 \times 0.6 \mathrm{~mm}$. Follicles in pairs, c. 4 $\mathrm{cm}$ long, terete, narrowed at apex, glabrous. Seeds c. $5 \times 2.5 \mathrm{~mm}$, ovoid, flat, marginate; coma c. $6 \mathrm{~mm}$ long, silky white.

Flowering \& fruiting: July-October.

Habitat: Grows along hill slopes among grasses in association with Euphorbia antiquaorum L. and Senecio bombayensis N.P. Balakr.

Distribution: Endemic to northern Western Ghats of Maharashtra, India.

Specimens examined: INDIA, Maharashtra, Pune district, Junnar, Ralegaon Shinde, s.d., S.R. Yadav 5812; Ralegaon Shinde (grown in garden), 19.09.2011, S.S. Kambale \& A.A. Adsul SUK 2600; Ibid., 08.10.2012, S.S. Kambale \& A.A. Adsul SSK 25; Ibid., 19.09.2013, S.S. Kambale \& A.A. Adsul SSK 91 (SUK!).

Conservation status: Nayar and Sastry (1987) assessed it as Endangered while Mishra \& Singh (2001) considered as Critically Endangered. In the present study only 10 individuals were observed and the species is assessed here as Critically Endangered [CR: B2 a, b (iii, iv, v), c (iv)].

Notes: Ceropegia mahabalei is unique in having longest flowers in this genus. It closely resembles C. attenuata but can be distinguished by its corolla lobes shorter than tube, length of flowers, glabrous outer corona and non-divergent inner corona.

Ceropegia mohanramii S.R. Yadav, M.N. Gavade \& Sardesai, Rheedea 16(1): 33. 2006; Karthik. et al., Fl. Pl. India 1: 163. 2009; Kambale \& S.R. Yadav, Asklepios 115: 36. 2013. Type: INDIA, Maharashtra, Sindhudurg district, Kudal Tehsil, Kochara, 29.07.2000, M.M. Sardesai 2541 (holo CAL!; iso K!, SUK!, BLAT, BSI!).

Fig. 55

Perennial erect herbs. Rootstock tuberous; tubers $2-4 \mathrm{~cm}$ in diam., slightly depressed globose; roots fibrous. Stem c. $24 \mathrm{~cm}$ long, terete, $4-7 \mathrm{~mm}$ in diam., usually unbranched, internodes $0.5-3.2 \mathrm{~cm}$ (lower internodes $c .3 .2 \mathrm{~cm}$, upper $c .0 .5 \mathrm{~cm}$ ), puberulous, 

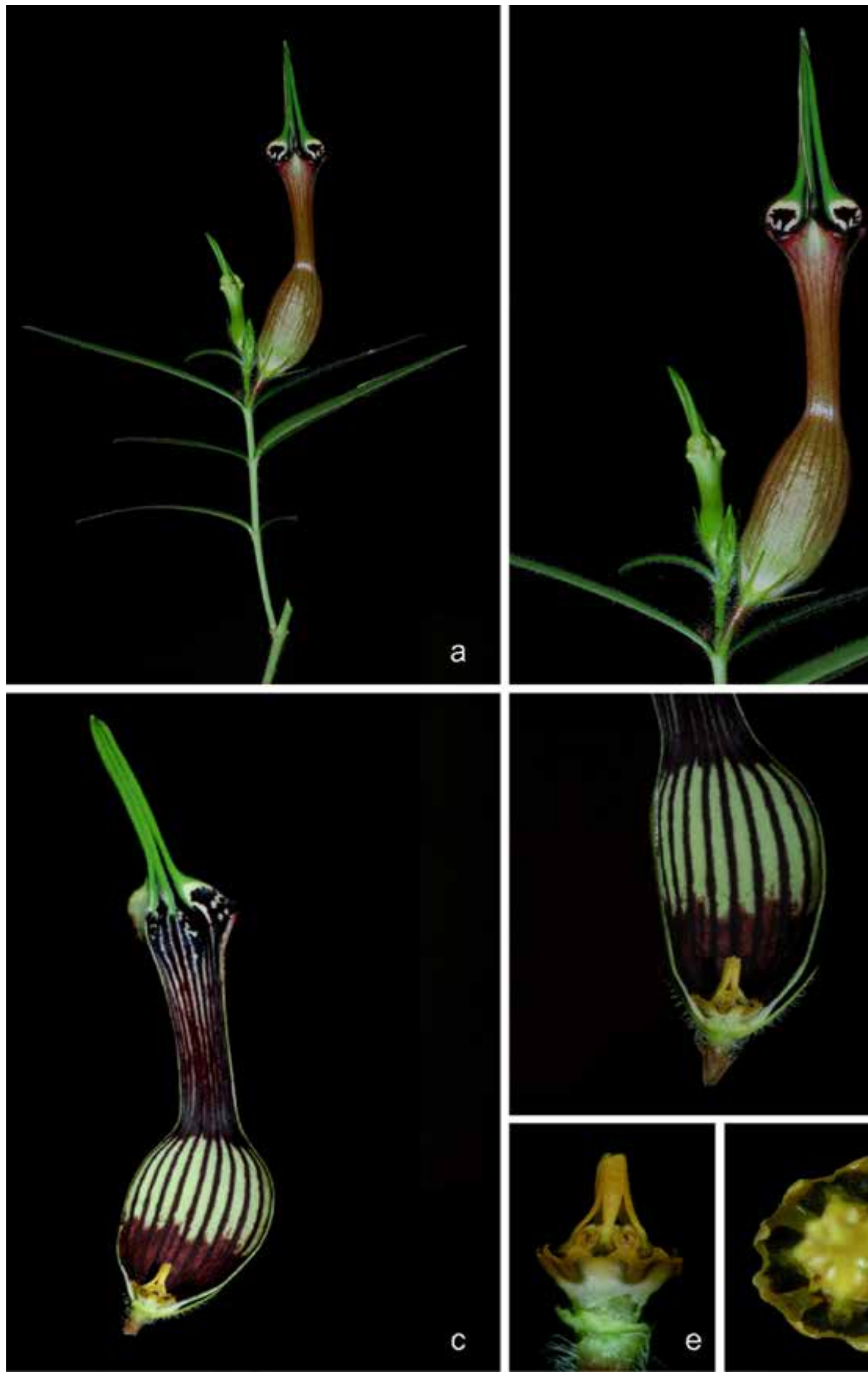

Fig. 54. Ceropegia mahabalei Hemadri \& Ansari: a. Habit; b. Flower; c. Flower L.S.; d. Flower L.S.-close-up; e. Corona-lateral view; f. Coronatop view. 
sparingly hairy, greenish-white. Leaves sessile to sub-sessile; lamina 1.8-5.5 × 0.3-0.6 cm (lower ones small, middle large and terminal small), linearlanceolate, occasionally falcate, channeled above, midrib puberulous beneath, glabrous beneath, except for midrib, ciliolate along margins. Flowers solitary, extra-axillary; peduncles 1-2 mm long, terete, puberulous; bracts $1.5-2 \times 0.3-0.5 \mathrm{~mm}$, solitary, linear, acute, glabrous; pedicels $8-8.5 \times$ 0.5-1 mm, slender, terete, pubescent. Sepals 3-3.5 $\times 0.1-0.15 \mathrm{~mm}$, linear-subulate, acuminate, hairy along midnerve up to middle of lobe; tube 3.2$3.5 \mathrm{~cm}$ long, $c$. half or $2 / 3^{\text {rd }}$ part of corolla dilated, glabrous, green throughout, with slight yellow tinge; tube $2.4-2.6 \mathrm{~cm}$ long, with 5 distinct lines, slightly curved, pale purple within dilated part and striated with purple lines up to the throat; tube rarely hairy in the dilated part within, glabrous otherwise; lobes linear-oblong (at base), 0.8-1 $\mathrm{cm}$ long, reflexed at base, connate at tip forming ampuliform cage, finely pubescent along margins and within, sometimes blotched with purple small dots/lines within. Corona bi-seriate, stipitate; outer of 5 shortly bifid lobes, bowl-shaped, densely ciliate along margins and within; inner of 5 erect, erecto-divergent linear lobes, 2.5-3 mm long, yellow blotched with purple. Pollinarium $0.6 \times 0.4$ $\mathrm{mm}$, pollinia broadly elliptic. Follicles usually in pairs, rarely single, $3.2-6.4 \times 0.2-0.3 \mathrm{~cm}$, straight, slightly curved on drying, tapering at both ends, erect. Seeds $3-5 \times 1.5-1.7 \mathrm{~mm}$, ovate-oblong; coma 1-1.2 cm long.

Flowering \& fruiting: July-December.

Chromosome number: $2 n=22$ (Gosavi et al., 2012).

Habitat: Grows on low elevation lateritic plateaus in association with Flemingia nilgheriensis (Baker) T. Cooke and Wrightia tinctoria R.Br.

Distribution: Endemic to Maharashtra, India.

Specimens examined: INDIA, Maharashtra, Sindhudurg district, Kochara, Sriramwadi, 11.07.2010, S.S. Kambale SUK 2559; Sriramwadi (grown in garden), 16.07.2011, S.S. Kambale SUK 2576; 12.08.2013, S.S. Kambale SSK 66; 15.08.2014, S.S. Kambale SSK 267 (SUK!).

Conservation status: This plant is known only from the type locality in Sindhudurg district of Maharashtra. It is assessed here as Critically
Endangered [CR: B2 a, b (iii, iv, v)].

Notes: Ceropegia mohanramii is closely similar to $C$. attenuata but differs in its shorter corolla lobes and ampuliform cage.

Ceropegia nampyana Manudev, Kambale \& Pramod, Int. J. Advanced Res. 4(5): 1408. 2016. Type: INDIA, Kerala, Kozhikode district, $25 \mathrm{~km}$ from Kozhikode city, Nanminda, Pokkunnu, 13.09.2014, Manudev, Pramod \& Prakash 138969 (holo CALI!; iso DEV!, SUK!).

Fig. 56

Perennial erect herbs. Rootstock tuberous; tubers 1.5-2 cm across, globose-discoid; roots fibrous. Stem c. 9-15 cm high, c. $3 \mathrm{~mm}$ across, terete, unbranched, pubescent. Lamina 4.5-10 × 0.8-1 $\mathrm{cm}$, linear to elliptic-lanceolate, falcate, acute at apex, narrowed at base, glabrous, margins serrulate, strigose towards base; petioles 4-6 mm long, channeled above, glabrous. Flowers solitary, extra-axillary; peduncles 1.5-2 mm long, terete, glabrous; bracts solitary, $1.5-2 \mathrm{~mm}$ long, linear, glabrous; pedicels $0.8-1 \mathrm{~cm}$ long, terete, glabrous. Sepals 2.5-3.5 mm long, linear-subulate, glabrous. Corolla $c .3 \mathrm{~cm}$ long; tube c. $2 \mathrm{~cm}$ long, slightly curved, dilated at base, narrow at middle, funnelshaped above, glabrous, pale green with purple bands at base, deep purple otherwise and deep purple within; lobes c. $1.3 \mathrm{~cm}$ long, shorter than the tube, twisted, acute, folded along the margins, connate at the tip with glandular trichomes along margins; trichomes clavate, purple and translucent; unicellular conical purple trichomes present within at the base of corolla lobes. Corona bi-seriate; outer of 5-bifid, lobes c. $0.5 \mathrm{~mm}$, bowl-shaped, c. $1.2 \times 2.5$ $\mathrm{mm}$, hairy along margins and within; inner erect, linear, c. $2.5 \mathrm{~mm}$ long, alternate with outer corona, whitish-cream. Pollinia yellow, attached to brown pollen carriers by short caudicles. Pollinarium $c$. $0.35 \times 0.45 \mathrm{~mm}$. Follicles in pairs, $6-7 \times c .0 .3 \mathrm{~cm}$, tapering towards apex, usually unequal, glabrous. Seeds c. $5 \times 3 \mathrm{~mm}$, ovate-oblong, comose; coma $c$. $2 \mathrm{~cm}$ long.

Flowering \& fruiting: August-October.

Habitat: Grows along lateritic hills in association with Canscora pauciflora Dalzell, Crotalaria nana Burm.f., Cyanotis burmanniana Wight, Eriocaulon eurypeplon Korn., Gloriosa superba L., Ischaemum ciliare Retz., Justicia japonica Thunb., Murdannia semiteres (Dalzell) Santapau, Pogostemon quadrifolius 

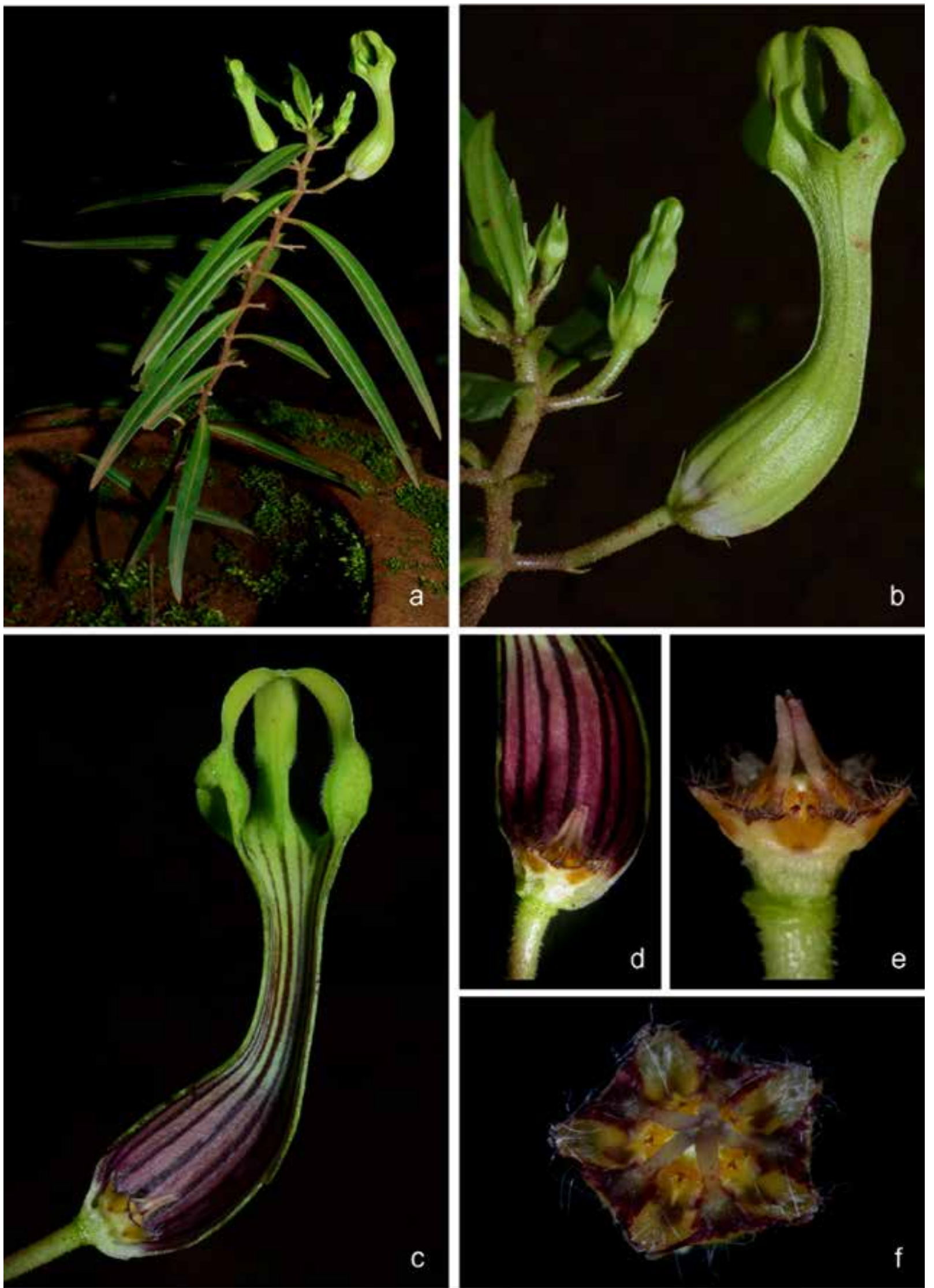

C

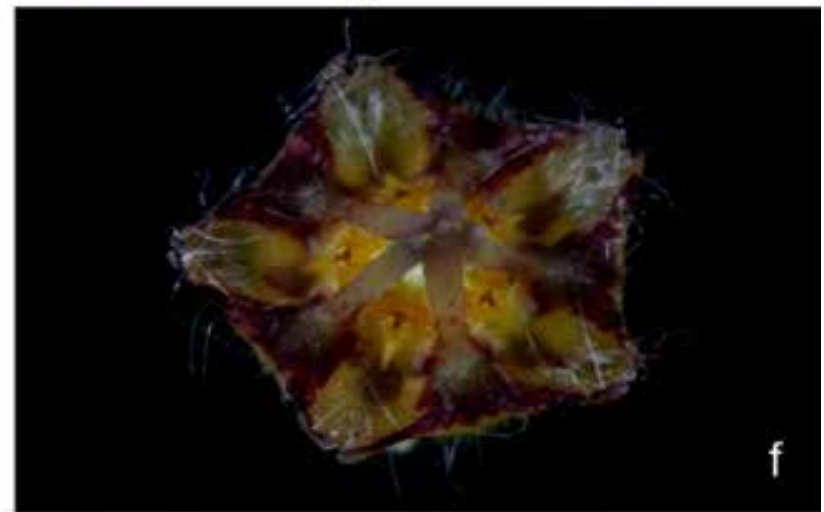

Fig. 55. Ceropegia mohanramii S.R. Yadav, M.N. Gavade \& Sardesai: a. Habit; b. Flower; c \& d. Flower L.S.; e. Corona-lateral view; f. Corona-top view. 

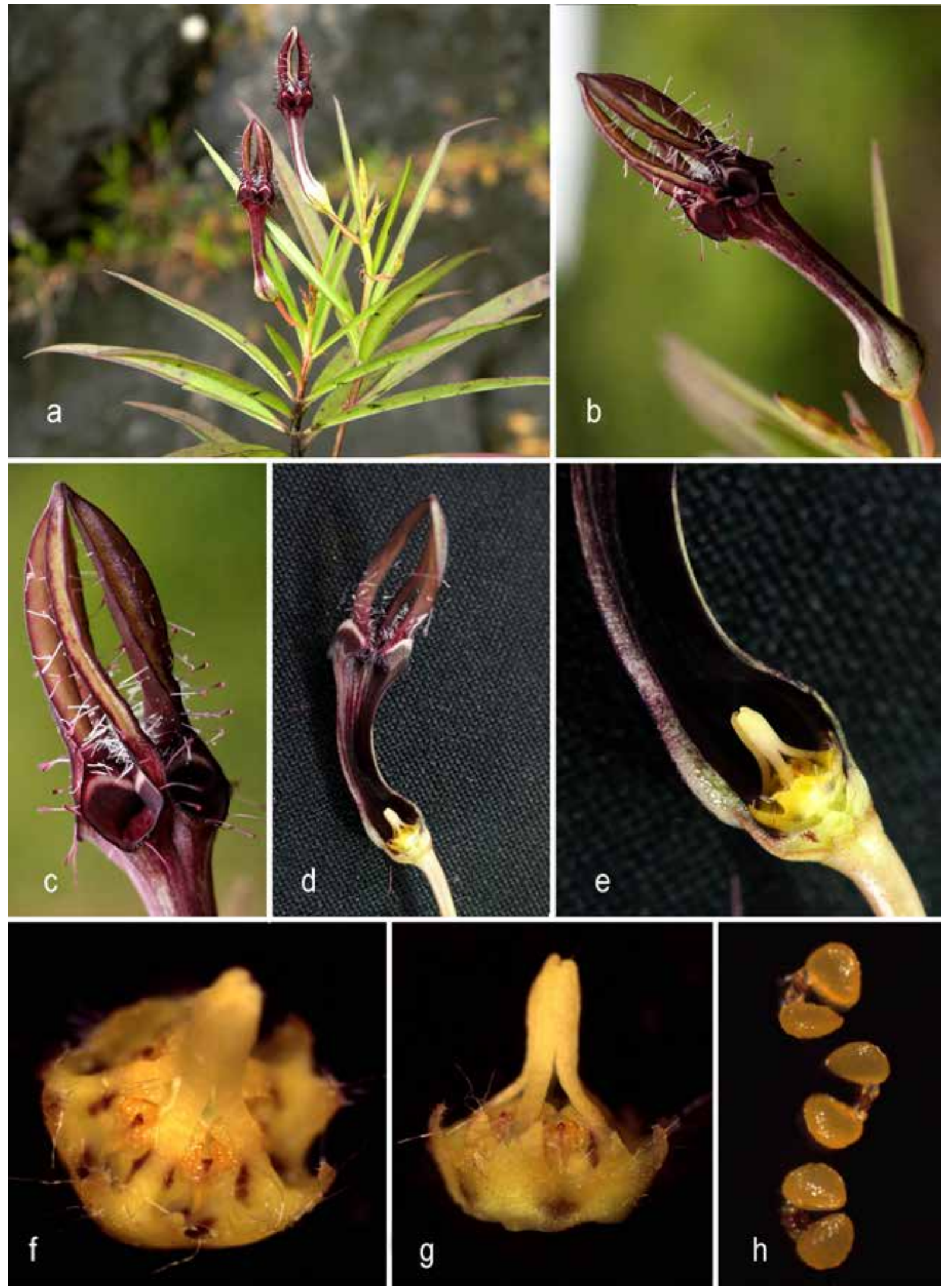

Fig. 56. Ceropegia nampyana Manudev, Kambale \& Pramod: a. Habit; b. Flower; c. Corolla lobes-enlarged view; d \& e. Flower L.S.; f. Corona-top view; g. Corona-lateral view; h. Pollinaria. 
(Benth.) F. Muell. and Polycarpaea corymbosa (L.) Lam.

Distribution: Hitherto known only from the type locality, Kerala, India.

Conservation status: Data Deficient [DD] (Manudev et al., 2016).

Notes: Ceropegia nampyana is close to C. spiralis Wight but differs by having corolla lobes shorter than the tube, corolla tube glabrous within, hairy outer corona as opposed to corolla lobes as long as corolla tube, bulbous based conical trichomes present within tube, glabrous outer corona in $C$. spiralis. The unicellular glandular trichomes and clavate translucent trichomes on corolla lobes in these two taxa are unusual in the series Attenuatae.

Ceropegia noorjahaniae Ansari, J. Bombay Nat. Hist. Soc. 69(1): 250. 1972; Ansari, Fasc. Fl. India 16: 26. 1984; M.P. Nayar \& Sastry, Red Data Book Indian Pl. 2: 52. 1988; S.D. Deshp. et al., Fl. Mahabaleshwar, Maharashtra 1: 361. 1993; A.P. Jagtap \& N.P. Singh, Fasc. Fl. India 24: 233. 1999; D.K. Mishra \& N.P. Singh, Endem. Threat. Pl. Maharashtra 142. 2001; A.P. Jagtap \& Das Das in N.P. Singh et al., Fl. Maharashtra, Dicot. 2: 353. 2001; M.R. Almeida, Fl. Maharashtra 3A: 235. 2001; F. Albers \& Meve, Ill. Handb. Succ. Pl. Asclepiadaceae 92. 2002; Karthik. et al., Fl. Pl. India 1: 163. 2009; Kambale \& S.R. Yadav, Asklepios 115: 36. 2013. Type: INDIA, Maharashtra, Satara district, Wai-Panchgani Ghat, 05.08.1970, M.Y. Ansari 104880A (holo CAL!; iso CAL!, BSI!, K, BLAT, L, MH, LE, MO; para BSI!)

Fig. 57

Perennial erect herbs, occasionally twining. Rootstock tuberous; tubers $1-3.5 \mathrm{~cm}$ in diam., depressed globose to discoid; roots fibrous. Stem slender, minutely pubescent when young, glabrous when mature, 12-100 cm long. Leaves sub-sessile-petiolate; lamina linear-lanceolate, $3.1-6.5 \times 1.1-1.7 \mathrm{~cm}$, pubescent above and along margins, glabrous beneath except for midnerve, dark green above, pale below; petioles 0.5-1.2 $\mathrm{cm}$, channeled above, hairy along the margins, glabrous otherwise. Cymes 3-flowered, extraaxillary; peduncles 7-9 mm long, slender, glabrous; bracts solitary, c. $2.5 \mathrm{~mm}$ long, linear; pedicels 4-7 $\mathrm{mm}$ long, slender, glabrous. Sepals 2.5-3 mm long, subulate, glabrous. Corolla $1.7-2.2 \mathrm{~cm}$ long, tube $1.2-1.3 \mathrm{~cm}$ long, slightly curved, inflated at base, glabrous, pale green outside in lower $3 / 4^{\text {th }}$ part, pale to dark purplish brown in upper $1 / 4^{\text {th }}$ part up to the basal part of corolla lobes, green striated within, glabrous; lobes 7-9 mm long, linear, oblong, with acute apex and deltoid base, connate at the tips forming an ovoid cage, slightly reflexed all along the length, glabrous, greenish above, pale to dark purplish-brown near base. Corona bi-seriate, outer of 5-bifid or deeply emarginated, saucer to bowlshaped, 2-3 mm long, ciliate along the margins, greenish-yellow, purple along the margins; inner of 5 erect lobes, c. $3 \mathrm{~mm}$ long, glabrous, deep purple at the base, straight at the tips. Follicles 5.5$9 \mathrm{~cm}$ long, unequal, tapering towards both ends, glabrous. Seeds c. $3.5 \times 2.5 \mathrm{~mm}$, ovate, marginate, comose; coma c. $2 \mathrm{~cm}$ long, silky white.

Flowering \& fruiting: July-September.

Chromosome number: $2 n=22$ (Gosavi et al., 2012).

Habitat: Grows in well drained soil amidst grasses along hill slopes of dry deciduous forests.

Distribution: Endemic to Maharashtra, India.

Specimens examined: INDIA, Maharashtra, Nanded district, Ambadi, 07.09.1979, B.R. Zate 922 (BAMU!); Satara district, Amberi, s.d., S.P. Gaikwad 532; Kartikswami hills, 28.08.1989, S.R. Yadav 5914; Ibid., 22.08.1999, H.S. Patil 3514; Ibid., (grown in garden), 04.08.2013, S.S. Kambale SSK 58; Ibid., 06.07.2014, S.S. Kambale SSK 237; Ibid., 21.07.2004, M.Y. Kamble 2143; Khambataki Ghat, 15.08.2003, M.Y. Kamble 2129; Khindwadi, 10.1995, M.P. Bachulkar-Cholekar 5800; Pasarani Ghat, 02.09.2005, M.Y. Kamble 2177; Wai-Pasarani Ghat, 16.08.2011, S.S. Kambale SUK 2592 (SUK!).

Conservation status: Nayar and Sastry assessed it as Rare while Mishra and Singh (2001) considered it as Endangered. It is represented with very few individuals in its natural habitat. Habitat loss and food value of tuber are the major threats to the species. In present study, it is assessed as Critically Endangered [CR: B2 a, b (iii, iv)].

Notes: Ceropegia noorjahaniae is closely similar to C. fimbriifera and C. bhatii, but differs in having abruptly dilated corolla tube and ovoid cage of corolla lobes.

Ceropegia pusilla Wight \& Arn., Contr. Bot. India 31. 1834; Decne. in DC., Prodr. 8: 643. 1844; Wight, Icon. Pl. Ind. Orient. 4, t. 1261. 
Kambale \& Yadav 109
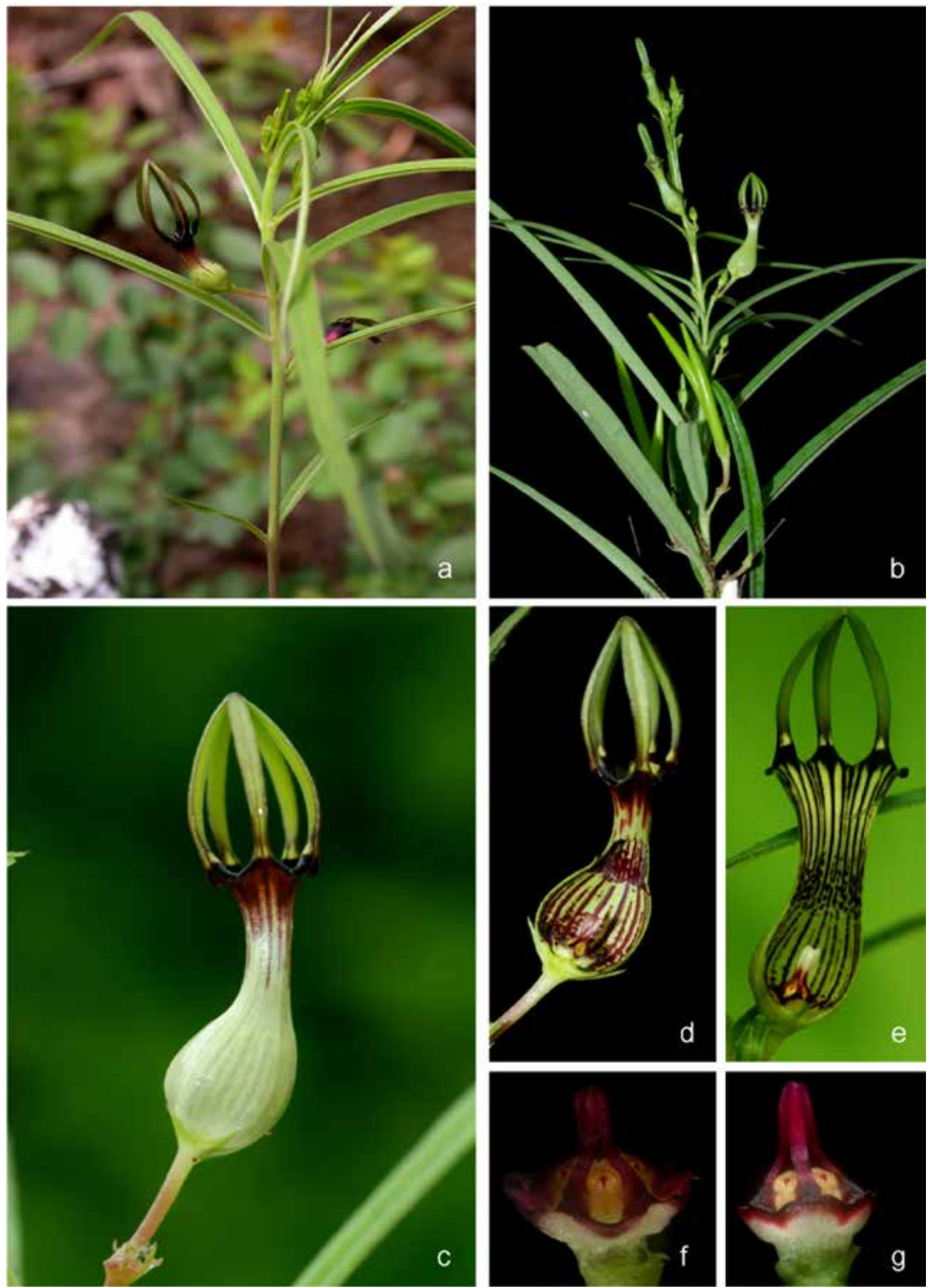

Fig. 57. Ceropegia noorjahaniae Ansari: a \& b. Habit; c. Flower; d \& e. Flower L.S.; f \& g. Corona-lateral view. 
1848; Hook.f., Fl. Brit. India 4: 66.1883. Fyson, Fl. Nilgiri \& Pulney 2: 191. 1915; Gamble, Fl. Madras 4: 856. 1921; H. Huber, Mem. Soc. Brot. 12: 53. 1957; B.D. Sharma et al., Fl. Karnataka, Analysis 166. 1984; Ansari, Fasc. Fl. India 16: 28.1984; M.P. Nayar \& Sastry, Red Data Book Indian Pl. 1: 68. 1987; S.R. Sriniv. in Henry et al., Fl. Tamil Nadu Ind., Ser I: Analysis 2: 84. 1987; S.M. Almeida, Fl. Savantwadi 1: 258. 1989; A.P. Jagtap \& N.P. Singh, Fasc. Fl. India 24: 236. 1999; F. Albers \& Meve, Ill.Handb. Succ. Pl. Asclepiadaceae 96. 2002; Karthik. et al., Fl. Pl. India 1: 163. 2009. Lectotype (designated by Singh, 2015): INDIA, Tamil Nadu, Neelgherries (Nilgiri), s.d., R. Wight cat. n. 1514 (E00179559 image!).

Fig. 58

Perennial erect herbs. Rootstock tuberous; tubers $2-2.6 \mathrm{~cm}$ in diam., globose; roots fibrous. Stem 3-17 cm long, usually single from an individual tuber, rarely two, glabrous. Leaves sessile, crowded at apex; lamina $2.2-5.5 \times 0.3-0.5 \mathrm{~cm}$, linearlanceolate-spathulate, channeled above, acute at apex, narrow at base, glabrous. Flowers solitary, extra-axillary; pedicels c. $2 \mathrm{~cm}$ long, puberulous. Sepals linear, glabrous, purplish. Corolla $1.3-2.8 \mathrm{~cm}$ long, glabrous; tube $0.8-2 \mathrm{~cm}$ long, dilated at base, cylindrical otherwise, glabrous within, striated with deep purple bands outside, purple within at base, pale at above; lobes 4-8 $\mathrm{mm}$ long, linearoblong, sub-spathulate, connate at tip forming ovoid cage, glabrous. Corona bi-seriate; outer of 5-bifid, narrowly deltoid, ciliate along margins, brown lobes; inner erect, linear, glabrous. Follicles c. $4.6 \mathrm{~cm}$ long, swollen towards base, narrow at apex, glabrous. Seeds comose, ovoid.

Flowering \& fruiting: June-September.

Habitat: Grows amidst grasslands of hill slopes.

Distribution: South India (Karnataka, Kerala, Tamil Nadu). Report by Almeida (1996) is doubtful since no specimens have been located during this study from the mentioned Amboli, Maharashtra.

Chromosome number: $n=11 \& 2 n=22$ (Sreedevi \& Namboodiri, 1977).

Specimens examined: INDIA, Kerala, Idukki district, Munnar, Kuttiay valley, 29.07.1982, Nambiar \& Sasidharan 1860 (KFRI!). Tamil Nadu, Madurai district, Meghamalai, Varaiyattumottai, 28.05.1989, A. Rajendran 86776 (MH!); Nilgiris district, way to
Nilgiri peak, Mudimundu, 26.10.1972 J.L. Ellis 43388; Nilgiri Peak Reserve Forest, 14.05.1971, J.L. Ellis 38460; Ooty, Nanjanad, 17.09.1930, V. Narayanaswami 4429; Pakasuramalai, 29.08.1957, K.M. Sebastine 3933; view point, Kodanad, 12.11.1970, E. Vajravelu 36855 (MH!).

Ceropegia spiralis Wight, Icon. Pl. Ind. Orient. 4(1): 15, t. 1267. 1848; Hook.f., Fl. Brit. India 4: 66. 1883; Gamble, Fl. Madras 4: 856. 1921; H. Huber, Mem. Soc. Brot. 12: 127. 1957; K.M. Matthew, Fl. Tamil Nadu Carnatic 2: 937. 1983; Ansari, Fasc. Fl. India 16: 30. 1984; B.D. Sharma et al., Fl. Karnataka, Analysis 166. 1984; M.P. Nayar \& Sastry, Red Data Book Indian Pl. 1: 71. 1987; S.R. Sriniv. in Henry et al., Fl. Tamil Nadu Ind., Ser I, Analysis 2: 84. 1987; Manilal, Fl. Silent Valley 177. 1988; Hanumanthappa in Pull. \& Moulali, Fl. Andhra Pradesh 2: 586. 1997; A.P. Jagtap \& N.P. Singh, Fasc. Fl. India 24: 239. 1999; F. Albers \& Meve, Ill. Handb. Succ. Pl. Asclepiadaceae 101. 2002; T.S. Nayar et al., Fl. Pl. Kerala 87. 2006; Karthik. et al., Fl. Pl. India 1: 164. 2009; Kambale \& S.R. Yadav, Asklepios 115: 38. 2013. Lectotype (designated by H. Huber, 1957): INDIA, Kerala, Palakkad Hills (Balaghat hills near Madras), s.d., R. Wight s.n. (K000894294 image!).

Ceropegia munronii Wight, Icon. Pl. Ind. Orient. 4: 15, t. 1264. 1848. Lectotype (designated by Kambale
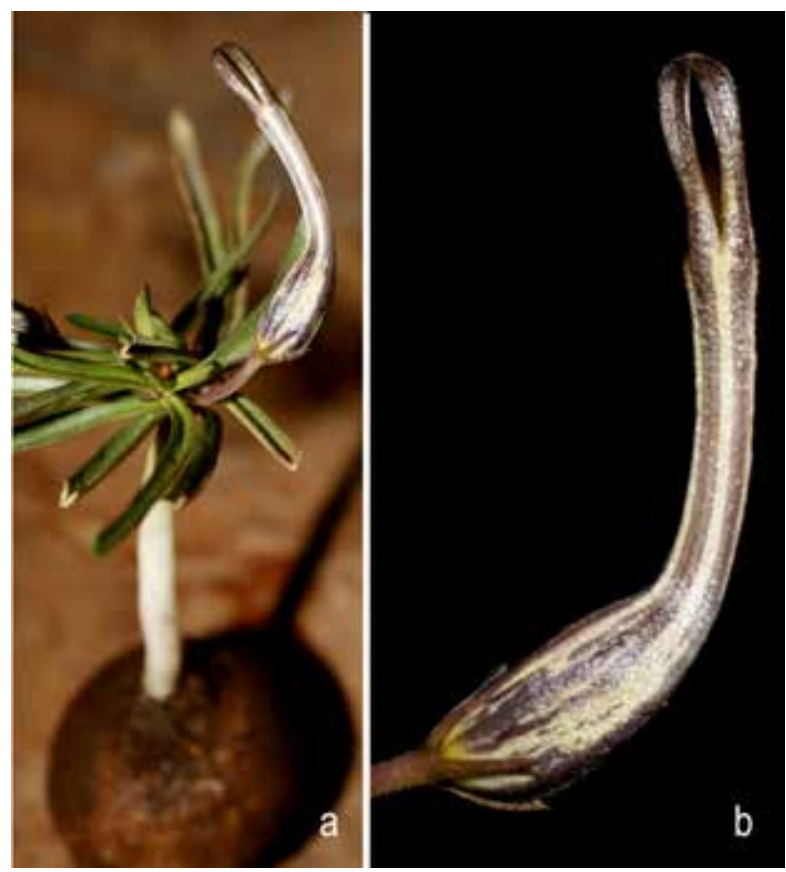

Fig. 58. Ceropegia pusilla Wight \& Arn.: a. Habit; b. Flower (Photos Dr. Nilesh Malpure). 
\& Yadav, 2015): Wight, Icon. Pl. Ind. Orient. t. 1264. 1848.

Fig. 59

Vernacular name: Amruthanjana gadde (Kannada).

Perennial erect herbs. Rootstock tuberous; tubers 2-10.5 cm diam., globose-discoid; roots fibrous. Stem c. 7-30 cm long, terete, unbranched, pubescent. Lamina $2.3-12 \times 0.2-1.2 \mathrm{~cm}$, linear to elliptic-lanceolate, falcate, acute at apex, narrowed at base, glabrous; petioles 4-6 mm long, channeled above, glabrous. Flower solitary, extra-axillary; peduncles 2-3 mm long, terete, glabrous; bracts solitary, c. $3 \mathrm{~mm}$ long, linear, glabrous; pedicels 5-7 mm long, terete, glabrous. Sepals 4-5 mm long, subulate, glabrous. Corolla $4.6-6.3 \mathrm{~cm}$ long; tube $1.2-2 \mathrm{~cm}$ long, curved in almost $90^{\circ}$ to the axis, much dilated at base, narrow at the middle, funnel-shaped at throat, bulbous based conical trichomes present throughout tube within, dilated portion glabrous within, cream coloured, mottled with pink, deep purple band within; lobes 3.6-4.2 $\mathrm{cm}$ long, spirally coiled, acute, reflexed, connate at the tip forming spiral cage, cylindrical and hollow clavate trichomes present along margins, unicellular clavate trichomes present within at the base of corolla lobes. Corona bi-seriate; outer of 5-bifid lobes, c. $1.5 \times 3 \mathrm{~mm}$, saucer-shaped, glabrous along margins, hairy within; inner erect, linear, c. $3 \mathrm{~mm}$ long, alternate with outer corona, whitish-yellow with purple tinge at tip. Follicles $18-19 \mathrm{~cm}$ long, tapering towards apex, usually unequal, glabrous. Seeds comose; coma silky white.

Flowering \& fruiting: August-February.

Chromosome number: $2 n=22$ (Gosavi et al., 2012).

Habitat: Grows along hill slopes in association with grasses. It also grows at drier hill tops and plains in association with succulent Euphorbias.

Distribution: India (Andhra Pradesh, Karnataka, Kerala, Tamil Nadu).

Specimens examined: INDIA, Andhra Pradesh, Annamayya Project, 29.09.2007, B. Sadasivaiah \& S.K. Basha 30642 (SKU!); Chittoor district, Somala, 10.02.2008, M.V. Suresh Babu 31158 (SKU!); Cuddapah district, Cuddapah hills, 1881, R.H. Beddome s.n. (MH!); Guntur district, Bollapalli, 16.07.2007, B.R.P. Rao \& B. Sadasivaiah 29580 (SKU!). Karnataka; Bangalore, Bannerghatta, 12.09.1978, T.M. Ramakrishna TMR 1327 (MH!);
Namada Chilume, 08.11.2013, M. Sanjappa, K. Ravikumar \& S.S. Kambale SSK 201 (SUK!); Tumkur district, Siddara Betta, 06.11.2013, S.S. Kambale, S.R. Yadav \& A.R. Gholave SSK 100; Ibid., 11.10.2014, S.S. Kambale SSK 325 (SUK). Kerala, Thiruvanathapuram, Pallikondanparai, 11.10.1928, V. Narayanaswamy 991 (MH!). Tamil Nadu, Dharmapuri district, Devarbetta, behind forest rest house, 29.10.1981, K.M. Matthew, S.J. Britto \& N. Rani 28516 (RHT!); Madurai district, Madura, way to high wavy Ghats, 06.09.1925, K.R.C. Jacob 17526 (MH!).

Conservation status: Nayar and Sastry (1987) assessed it as Vulnerable. After extensive survey in all possible localities of occurrence of the species, it has been observed that populations are represented by few individuals at each locality. A thorough study is required to assess its present status. Therefore it is treated here as Data Deficient (DD).

Notes: Ceropegia spiralis is similar to C. attenuata, but differs by its spirally coiled corolla lobes, basally dilated corolla tube hairy within and habitat, i.e. hills of drier parts of South India. It is also close to C. fimbriifera but differs from it in having spirally coiled corolla lobes. All these three species belongs to Ceropegia sect. Tiloris ser. Attenuatae by $\mathrm{H}$. Huber (1957). While describing the species, Wight (1848) did not provide any information regarding collection number and locality on the specimen. Huber (1957) inadvertently designated a lectotype by citing "Typus: Wight in Herb. K."

\section{Acknowledgements}

Authors thank Dr. K.N. Gandhi (HUH) for critical comments on nomenclature; Dr. M.M. Lekhak, Dr. R.V. Gurav (SUK), Dr. D.J. Goyder (K), Dr. Peter Bruyns (CT), Dr. Masinde (Kenya) and Prof. Meve (Bayreuth University, Germany) for discussions; Prof. M. K. Janarthanam, Dr. Siddharthan, Prof. Pullaiah, Dr. Gajurel, Dr. Ravikumar, Dr. Sujanapal, Dr. Punekar, Dr. Debabrata Maity, Prof. Deepthi Yakandawala, Dr. Achala Attanayake and Mr. Joju Alappat (Director), Forest Research Centre, Wimbaligunj, South Andaman for various helps; authorities of the herbaria ARUN, ASSAM, BAMU, BLAT, BM, BSI, BSID, CAL, CALI, E, FRLH, G, GH, MH, PBL, PDA, K, KFRI, RHT, SUK, SKU, TBGT and Goa University Herbarium for permission 


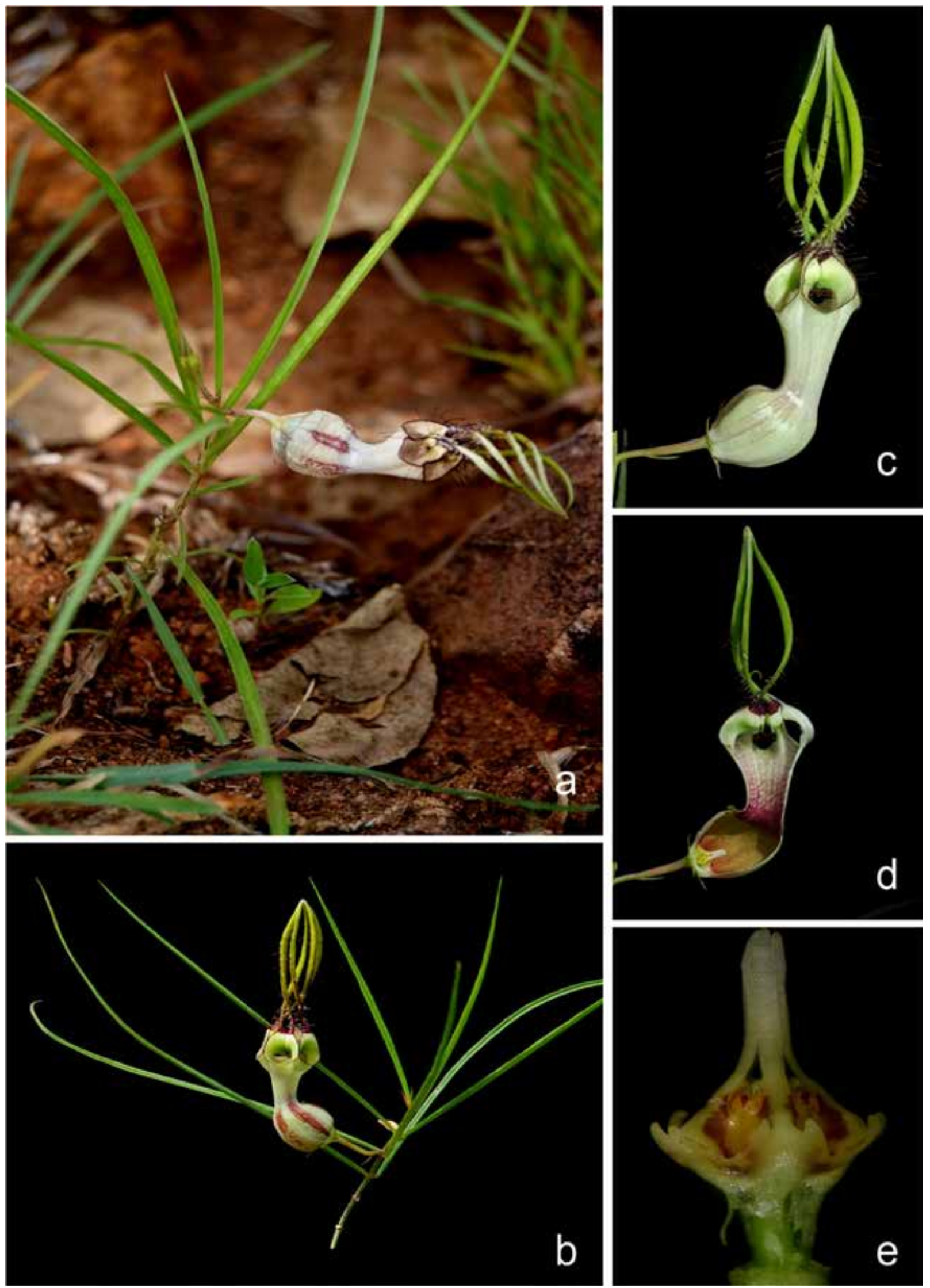

Fig. 59. Ceropegia spiralis Wight: a \& b. Habit; c. Flower; d. Flower L.S.; e. Corona-lateral view. 
to consult herbaria; Dr. M.M. Sardesai, Dr. V.B. Shimpale, Dr. G.G. Potdar, Dr. M.Y. Kamble, Dr. S.M. Shendage, Dr. N.V. Malpure, Dr. A.N. Chandore, Dr. K.V.C. Gosavi, Dr. Shrikant Sutar, Dr. Mayur Nandikar, Tushar Dixit and Dr. Avinash Adsul for support; Dr. Gnanasekharan, Dr. Navendu Page, Dr. Prabhukumar, Dr. K.M. Manudev, Dr. Kullayiswamy, Dr. Umesh Tiwari, Dr. K. Prasad, Miss. Laly Joseph, Mr. Abhishek Jain and Mr. Sushant More for photographs and Forest Departments of Andhra Pradesh, Arunachal Pradesh, Karnataka, Kerala, Maharashtra, Manipur and Tamil Nadu for permission and help. Thanks are due to anonymous reviewers for their critical comments which improved the quality of the text to great extent. S.S.K. thank University Grants Commission (UGC), Department of Biotechnology (DBT), New Delhi and District Planning and Development Committee, Kolhapur for financial assistance.

\section{Literature Cited}

AHMEDULLAH M. \& M.P. NAYAR 1987. Endemic Plants of the Indian Regions. Botanical Survey of India, Kolkata.

ALMEIDA M.R. 1996. Flora of Maharashtra. Volume 3A. St. Xavier's College, Mumbai. pp. 228-239.

ANSARI M.Y. 1971. Ceropegia vincifolia Hook. (Asclepiadaceae) from Maharashtra, its history and identity. Bulletin of the Botanical Survey of India 13(3 \&4): 187-191. https://doi.org/10.20324/nelumbo/ v13/1971/75667

ANSARI M.Y. 1984. Flora of India. Fascicle 16. Asclepiadaceae: Genus-Ceropegia. Botanical Survey of India, Kolkata.

BLATTER E. \& C. McCANN 1931. A new Ceropegia from the Western Ghats. Journal of the Bombay Natural History Society 34: 936.

BRUYNS P.V. 1997. A note on Ceropegia L. (Asclepiadaceae) of Silent Valley, Kerala, India. Rheedea 7(2): 107-114.

BRUYNS P.V. 2003. Three new succulent species of Apocynaceae (Asclepiadoideae) from southern Africa. Kew Bulletin 58: 427-435.

BRUYNS P.V. 2014. The Apocynaceae of Namibia. Strelitzia 34. South African National Biodiversity Institure, Pretoria. pp. 1-158.
BRUYNS P.V., KLAK C. \& P. HANACEK 2017. A revised, phylogenetically based concept of Ceropegia (Apocynaceae). South African Journal of Botany 112: 399-436. http://dx.doi.org/10.1016/j. sajb.2017.06.021

COOKE T. 1905. Flora of the Presidency of Bombay. Volume 2. Botanical Survey of India, Kolkata.

DANIEL P. \& P. UMAMAHESWARI 2001. The Flora of the Gulf of Mannar, southern India. Botanical Survey of India, Kolkata. 608 p.

DIWAKAR P.G. \& R.K. SINGH 2011. A new variety of Ceropegia attenuata Hook. (Asclepiadaceae) from Mookambika Wildlife Sanctuary, Karnataka, India. Indian Journal of Forestry 34(2): 209-212.

EDGEWORTH M.P. 1862. Florula Mallica. Journal of the Proceedings of the Linnean Society, Botany 6(24): 204-205.

FORMAN L. \& D. BRIDSON 1992. The Herbarium Handbook. Royal Botanic Gardens, Kew.

GIRI G.S., PRAMANIK A. \& H.J. CHOWDHERY 2008. Materials for the Flora of Arunachal Pradesh: Asteraceae, Ceratophyllaceae. Volume 2. Botanical Survey of India, Kolkata. 492 pp.

GOOD R. 1952. An atlas of the Asclepiadaceae. New Phytologist 51:198-209. https://doi. org/10.1111/j.1469-8137.1952.tb06126.x

GOSAVI K.V.C., KAMBALE S.S. \& S.R. YADAV 2012. Marhold (ed.). IAPT/IOPB chromosome data 14. Taxon 61(6): 1336-1345. https://doi.org/10.1002/ tax.616027

GRAHAM R. 1834. Ceropegia lushii. Mr. Lush's Ceropegia. Botanical Magazine 61: 3300.

HOOKER J.D. 1883. The Flora of British India. Volume 4. L.Reeve and Co., London.

HOOKER W.J. 1854. Ceropegia thwaitesii: Mr. Thwaites's Ceropegia. Curtis's Botanical Magazine 80: 4758.

HUBER H. 1957. Revision der Gattung Ceropegia. Memorias da Sociedadu Broteriana. Volume 12. Instituto Botanico da Universidade de Coimbra.

HUBER H. 1983. Asclepiadaceae. In: DASSANAYAKE M.D. \& F.R. FOSBERG (eds.), Revised Handbook to the Flora of Ceylon. Volume 4. Oxford \& IBH Publishing Co., New Delhi.

IUCN 2017. Guidelines for using the IUCN Red List categories and criteria. Version 13. Prepared by the 
Standards and Petitions Subcommittee of the IUCN Species Survival commission (accessed 26.01.2019).

KAMBALE S.S. \& S.R. YADAV 2012. On the identity and occurrence of Ceropegia gardneri Thwaites in India. Asklepios 114: 23-26.

KAMBALE S.S. \& S.R. YADAV 2014. Lectotypifications in Ceropegia (Ceropegieae: Apocynaceae). Rheedea 24(1): 27-28.

KAMBALE S.S. \& S.R. YADAV 2015. Lectotypifications and synonymy in Ceropegia (Apocynaceae: Ceropegieae). Kew Bulletin 70: 57(1-9). https://doi. org/10.1007/s12225-015-9608-3

KAMBALE S.S., A.N. CHANDORE \& S.R. YADAV 2012. Ceropegia concanensis, a new species (Apocynaceae: Ceropegieae) from Western Ghats, India. Kew Bulletin 67(4): 843-848. https://doi. org/10.1007/s12225-012-9406-0

KHANDAL D., MORE S., KATARIA G. \& S.S. KAMBALE 2018. Ceropegia lucida-rediscovery and new distribution record for Arunachal Pradesh, India. Current Science 113(11): 2077.

KIDYOO M. \& C. PALIYAVUTH 2017. Ceropegia digitiformis sp. nov. (Apocynaceae, Asclepiadoideae) from northeastern Thailand. Taiwania 62(1): 24-28. http://dx.doi.org/10.6165/tai.2017.62.24

KULLAYISWAMY K.R., SANDHYARANI S. \& S. KARUPPUSAMY 2013. Ceropegia pullaiahii sp. nov. (Apocynaceae, Asclepiadoideae) from India. Nordic Journal of Botany 31(2): 166-169. http://dx.doi. org/10.1111/j.1756-1051.2012.01557.x

KUMAR R., SHARMA S. \& M.D. DWIVEDI 2018. Ceropegia mizoramensis and C. murlensis (Asclepiadaceae)-Two new species from Northeast India with Phylogenetic and morphological evidence support. Taiwania 63(2): 163-170. http:// dx.doi.org/10.6165/tai.2018.63.163

LI P.T., GILBERT M.G. \& W.D. STEVENS 1995. Asclepiadaceae. In: WU Z.Y. \& RAVEN P.H. (eds.), Flora of China. Volume 16. Science Press \& St. Louis, Beijing. pp. 189-270.

LINNAEUS C. 1753. Species Plantarum. Volume 1. Laurentius Salvius, Stockholm.

MALPURE N.V., KAMBLE M.Y. \& S.R. YADAV 2006. A new Species of Ceropegia L. (Asclepiadaceae) from the Western Ghats of India with a note on series Attenuatae Huber. Current Science 91: 1140-1142.
MANUDEV K.M., KAMBALE S.S., PRAMOD C. \& P.S. PRAKASH 2016. A new species of Ceropegia (Apocynaceae: Ceropegieae) from a midland lateritic hill of Kerala, India. International Journal of Advanced Research 4(5): 1408-1414. http://dx.doi. org/10.21474/IJAR01/466

MEVE U. 2002. Ceropegia. In: ALBERS F. \& U. MEVE (eds.), Illustrated handbook of succulent plants: Asclepiadaceae. Springer, Berlin.

MEVE U. 2009. Ceropegia thailandica (AsclepiadoideaeCeropegieae), a spectacular new Thai species. Bradleya 27: 161-164. http://dx.doi.org/10.25223/ brad.n27.2009.a3

MISHRA D.K. \& N.P. SINGH (2001). Endemic and threatened flowering plants of Maharashtra. Botanical Survey of India, Kolkata.

NAVANEETHAM N. \& R. SAMPATHKUMAR 1984. Chromosome Number Reports LXXXII. Taxon 33: 126-134.

NAVANEETHAM N. 1981. Chromosome Number Reports LXXII. Taxon 30(3): 696.

NAYAR M.P. \& A.R.K. SASTRY 1987. Red Data Book of Indian Plants. Volume I. Botanical Survey of India, Kolkata.

PATEL S.K., PUNJANI B.L., DESAI P.R., PANDEY V.B., CHAUDHARY Y.S. \& P.N. JOSHI 2017. Additional record and conservation measures of Ceropegia odorata Nimmo ex J. Graham from Gujarat State, India. Journal of Threatened Taxa 9(8): 10618-10622. http://dx.doi.org/10.11609/ jott.3432.9.8.10618-10622

PRAIN D.J. 1900. Some new plants from eastern India. Journal of the Asiatic Society of Bengal. Part 2. Natural History 69(2): 170-171.

PRASAD K., VENU P. \& S. KALIAMOORTHY 2017. Ceropegia omissa H. Huber (Apocynaceae: Asclepiadoideae) on the verge of extinction. Current Science 112(11): 2189-2191.

PUNEKAR S.A., JAGTAP S.D. \& S.S. DEOKULE 2006. A new variety of Ceropegia oculata Hook. (Apocynaceae: Asclepiadoideae) from Satpuda hill ranges of Maharashtra, India. Current Science 91(9): 1142-1145.

PUNEKAR S.A., TAMHANKAR S.A., LAKSHMI NARASIMHAN P., KUMARAN K.P.N., RAUT 
A. \& S.K. SRIVASTAVA 2013. Systematics and molecular phylogenetic analysis of erect species of Ceropegia section Buprestis species from India. Nelumbo 55: 6-30.

RAGHAVANR.S.\& M.Y.ANSARI 1975. Chromosome numbers in the genus Ceropegia L. Current Science 44(23): 863-864.

RAHANGDALE S.S. \& S.R. RAHANGDALE 2012. Variety Novae of Ceropegia mahabalei Hemadri \& Ansari (Apocynaceae: Asclepiadoideae). Indian Forester 138(2): 201-203.

ROXBURGH W.1795. Plants of the coast of Coromandel. W. Bulmer \& Co., London.

SINGH R.K. 2015. Lectotypification of two names of Indian species of Ceropegia (Apocynaceae). Telopea 18: 305-308. http://dx.doi.org/10.7751/telopea8637

SINGH R.K., PATIL S. \& J.S. JALAL 2014. Resurrecting the type locality of Ceropegia odorata (Apocynaceae) after 175 years. Taprobanica 6(2): 79-82. http://dx.doi. org/10.4038/tapro.v6i2.7186

SREEDEVI P. \& A.N. NAMBOODIRI 1977. In IOPB chromosome number reports LVI. Taxon 26: 257-274.

SREEKUMAR P.V., VEENAKUMARI K. \& M. PRASHANTH 1998. Ceropegia andamanica (Asclepiadaceae). A new 'fly trap flower' from the Andaman Islands, India. Blumea 43(1): 215-217.

SRI RAMA MURTHY K., KONDAMUDI R., CHANDRASEKHARA REDDY M., KARUPPU SAMY S. \& T. PULLAIAH 2012. Checklist and conservation strategies of the genus Ceropegia in India. International Journal of Biodiversity \& Conservation 4(8): 304-305. http://dx.doi. org/10.5897//IJBC12.011

SUJANAPAL P., SALIM P.M., ANIL KUMAR N. \& N. SASIDHARAN 2013. A new Species of Ceropegia (Apocynaceae: Asclepiadoideae) from India with notes on rare and threatened Ceropegia in Nilgiris of Western Ghats. Journal of the Botany Research Institute of Texas 7(1): 341-345.

SWARUPANANDAN K. \& J.K. MANGALY 1992. A new species of Ceropegia (Asclepiadaceae) from India. Nordic Journal of Botany 12(6): 699-701. https://doi. org/10.1111/j.1756-1051.1992.tb01851.x

THE PLANT LIST 2013. VERSION 1.1. Published on the Internet: http://www.theplantlist.org/ (accessed
01.01.2019).

THWAITES G.H.K. 1860. Enumeratio plantarum zeylaniae: An enumeration of Ceylon Plants. Volume 3. Dulau Co., London.

TURLAND N.J., WIERSEMA J.H., BARRIE F.R., GREUTER W., HAWKSWORTH D.L., HERENDEEN P.S., KNAPP S., KUSBER W.H., LI D.Z., MARHOLD K., MAY T.W., MCNEILL J., MONRO A.M., PRADO J., PRICE M.J. \& G.F. SMITH (eds.) 2018. International Code of Nomenclature for algae, fungi, and plants (Shenzhen Code) adopted by the Nineteenth International Botanical Congress Shenzhen, China, July 2017. Regnum Vegetable 159. Koeltz Botanical Books, Glashütten. https://doi.org/10.12705/Code.2018

VENKATAREDDI B. 1968. Ceropegia lawii Hook.f. and C. panchganiensis Blatt. \& McCann. Wildenowia 5(1): 29-33.

VOGEL E.F. DE 1987. Manual of Herbarium Taxonomy: Theory and Practice. Rijks herbarium, Leiden.

WALLICH N. 1830. Ceropegia elegans, Beautiful Ceropegia. Curtis's Botanical Magazine 57: t.3015.

WALLICH N. 1831. Plantae Asiaticae Rariores. Volume 2. Richard Taylor, London.

WIGHT R. 1834. Contributions to the Botany of India. Parbury, Allen \& Co., London.

WIGHT R. 1848. Icones Plantarum Indiae Orientalis. Volume 4(1). P.R. Hunt, American Mission Press.

YADAV S.R. \& S.M. SHENDAGE 2010. Ceropegia bhatii, a new species of Apocynaceae: Ceropegieae from Karnataka, India. Kew Bulletin 65: 1-4. https:// doi.org/10.1007/s12225-010-9189-0

YADAV S.R., GAVADE M.N. \& M.M. SARDESAI 2006. A New Species of Ceropegia L. (Asclepiadaceae) from Konkan, Maharashtra, India. Rheedea 16(1): 33-36.

YADAV S.R., SARDESAI M.M. \& S.P. GAIKWAD 2004. Ceropegia anantii (Asclepiadaceae), a new species from Western Ghats, India. Journal of Bombay Natural History Society 101(1): 141-143. 